\title{
An Experimental and Numerical Study of Three- Dimensional Fatigue Damage in Carbon Fibre Reinforced Polymers
}

\author{
by \\ Naglaa ElAgamy \\ A thesis submitted to the \\ Faculty of Graduate and Postdoctoral Affairs \\ in partial fulfillment of the requirements for the degree of
}

\section{Doctor of Philosophy \\ in}

Mechanical Engineering

\author{
Carleton University \\ Ottawa, Ontario \\ (C) 2015, \\ Naglaa ElAgamy
}




\begin{abstract}
A phenomenological experimental study was presented for understanding the effect of cyclic loading, generally experienced by typical modern aircraft structures during flight, on the propagation of micro-mesoscopic damage in carbon fibre reinforced composite laminates. Testing was carried out by employing ultra-high resolution SkyScan 1173 XRmicro computed tomography to identify and assess damage progression during fatigue testing. It provided qualitative as well as quantitative assessments of the damage in the composites which supported the analytical investigations.
\end{abstract}

An in-house solution was developed for statistically analyzing the occurrence, frequency and geometry of cracks throughout the fatigue life. This methodology was used to process the data from scan for the purposes of visualizing damage initiation and propagation. Hence, quantitative analysis could be performed. Analysis resulted in the definition of fatigue crack growth rates, $d a / d n$ for each of the 3 orthogonal planes, which was interpreted in terms of the 3 damage modes; opening, in-plane shear and out-of-plane shear. By applying linear elastic fracture mechanics (LEFM) laws, strain energy release rates were calculated, while differentiating between modes II and III in a novel manner.

For verifying the parameters obtained, definite cracks were traced and analyzed. Finally, a methodology was implemented to import the damage model into a finite element analysis (FEA) tool to be used for crack growth analysis, and simulations were compared to experimental findings. 


\section{Acknowledgments}

I would never have been able to finish my dissertation without the blessing of God. I am grateful to the God for the good health and wellbeing.

I am extremely grateful to my supervisor, Professor Jeremy Laliberté, for sharing expertise and sincere and valuable guidance. He was always available and willing to help, and had constantly led me to strive for excellence. He had seriously taken a personal interest in my success and building my confidence. I take this opportunity to express gratitude to my cosupervisor, Professor John Goldak, who gave me the opportunity to join the program.

Many thanks as well to Professor Mike Munro for his help and support over years and to my dear supervisor late Professor Atef Fahim, may his soul rest in peace. Thanks to all my colleagues; Camille, Pedro, Sadeem, Taran and Alex, for their valuable help on my project.

I wish to express my sincere thanks to Carleton University for the financial support, to the National Research Council in Ottawa and to the Science and Technology Centre at Carleton University for the specimens' preparations. Special thanks to the laboratory technologist, Steve Truttman, for helping with the fatigue testing.

I am also indebted to my mother, Soheir. I really appreciate her consistent support and encouragement. Finally, words cannot express my gratitude, appreciation, dedication and love of my sons, Eyad and Youssef. They happily allowed me for long hours, which they deserved more, to work on my thesis. They encouraged and pushed me forward, never complained nor demanded the least of what I owe them. I admit so many debts to repay, but my sons' support goes beyond any intellectual loan. 


\section{Table of Contents}

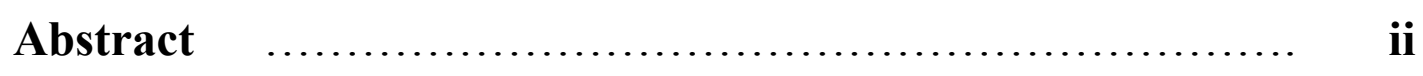

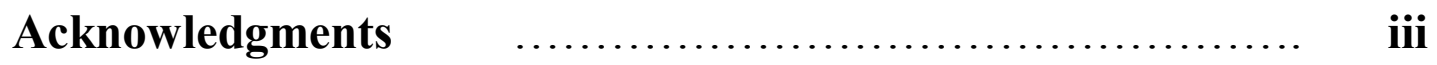

Table of Contents $\quad$................................... iv

List of Illustrations $\quad$................................... viii

List of Tables $\quad$......................................... xiv

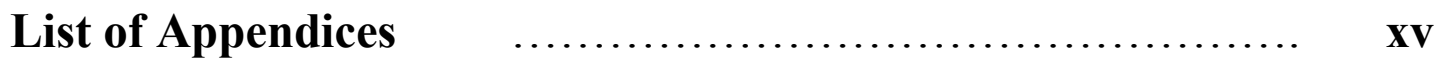

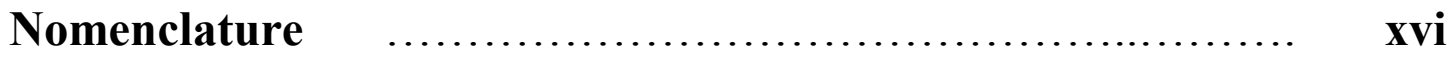

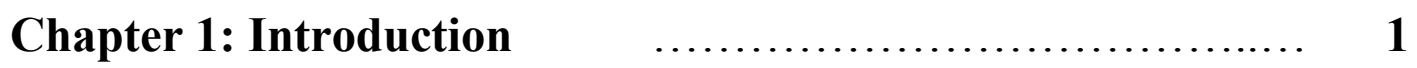

$1.1 \quad$ Background $\quad$............................................ 1

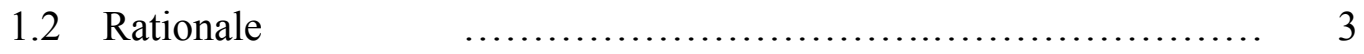

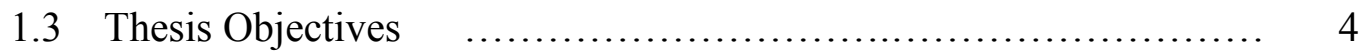

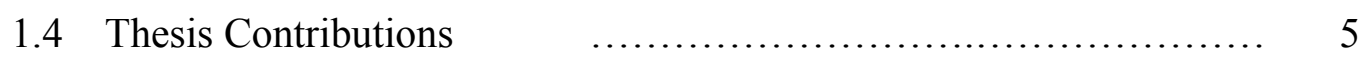

1.5 Research Methodology $\quad$....................................... 7

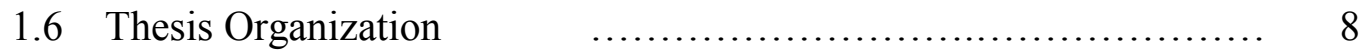

Chapter 2: Experimental Methodology $\quad \ldots \ldots \ldots \ldots \ldots \ldots \ldots \ldots . . . \ldots$

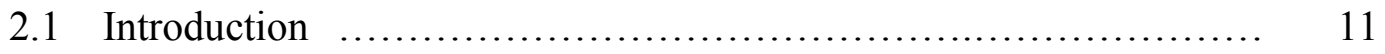

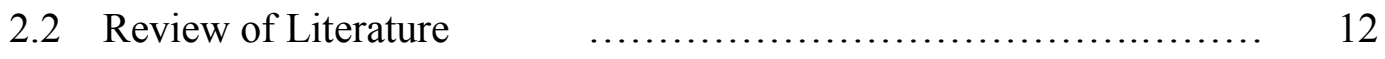

2.2.1 Geometrical Structure of Technical Textiles $\quad$............ 12

2.2.2 Geometrical Modelling of Technical Textiles $\quad \ldots \ldots \ldots \ldots . . . \quad 16$

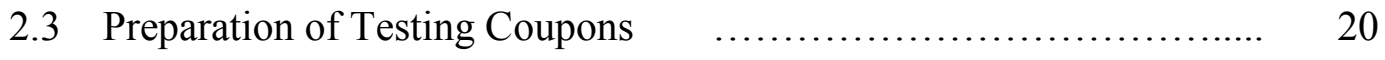

2.3.1 Material Specifications $\quad$................................. 21

2.3.2 Dimensions of Testing Coupons $\quad$........................ 22 
2.3.3 Quality Inspection of Testing Coupons $\quad \ldots \ldots \ldots \ldots \ldots \ldots . . \ldots 23$

2.3.4 Material Stiffness Properties $\quad$....................... 26

2.4 Mechanical Testing $\quad$.................................. 29

2.4.1 Identifying Static Strength $\quad \ldots \ldots \ldots \ldots \ldots \ldots \ldots \ldots \ldots . \ldots \ldots$

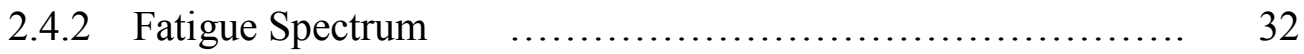

2.4.3 Fatigue Testing $\quad$.................................... 33

2.5 High Resolution X-ray Micro Computed Tomography $\quad$........... 35

\section{Chapter 3: Investigation of Fatigue Behaviour $\quad \ldots \ldots \ldots . \quad 37$}

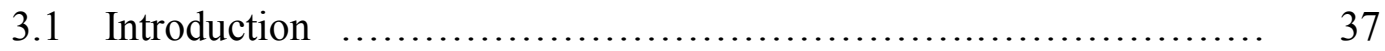

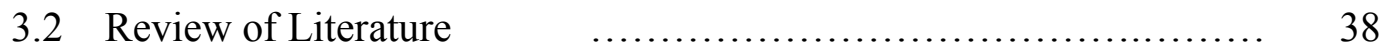

3.2.1 Implementation of Non-destructive Testing Tools $\quad \ldots . . . \ldots . . \quad 39$

3.2.2 Implementation of X-ray MicroCT Technique $\quad \ldots . \ldots \ldots . .40$

3.3 Qualitative analysis of 3D rendered images $\quad \ldots \ldots \ldots \ldots \ldots \ldots . . \ldots 4$

3.3.1 Rendered Volume Analysis $\quad \ldots \ldots \ldots \ldots \ldots \ldots \ldots \ldots . . \ldots 4$

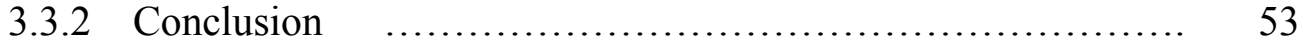

3.4 Proposed Procedure of Simulating Fatigue Damage by Using MicroCT

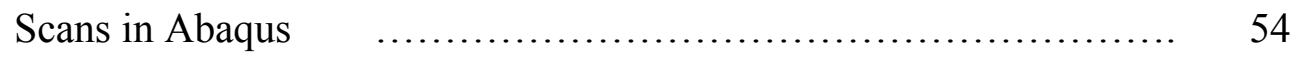

3.4.1 Virtual slicing .......................................... 55

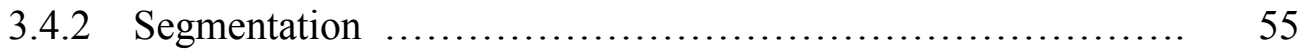

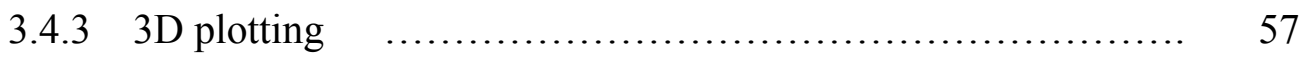

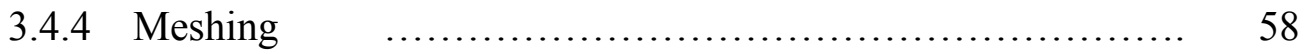

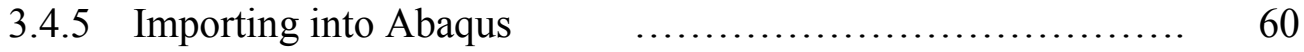

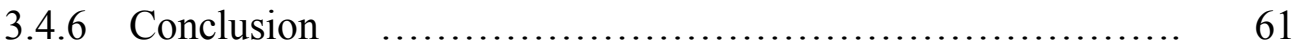


Chapter 4: Quantitative Analysis of Multimode Damage $\quad \ldots \quad 63$

4.1 Introduction $\quad$............................................ 63

4.2 Review of Literature $\quad$................................... 64

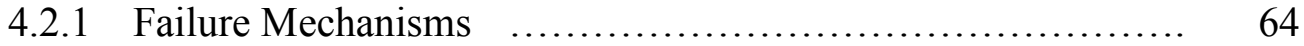

4.2.2 Failure Criteria $\quad$.................................... 66

4.2.3 Linear Elastic Fracture Mechanics $\quad$......................... 67

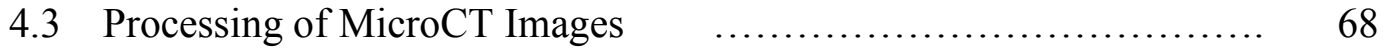

4.4 Segmentation of Voids $\quad$...................................... $\quad 70$

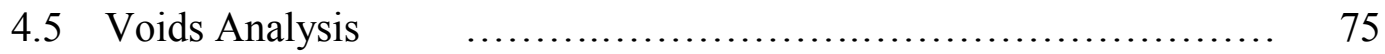

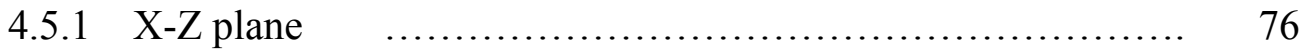

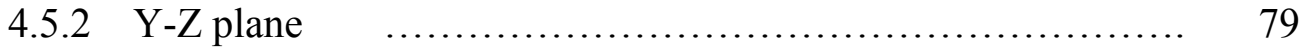

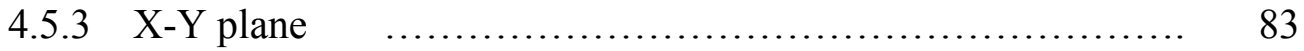

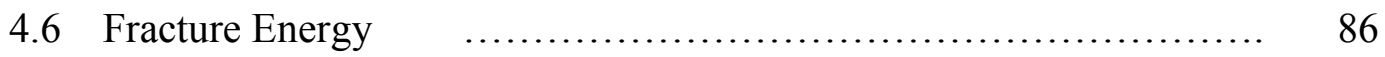

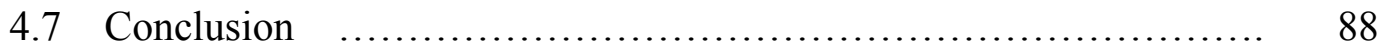

Chapter 5: Verification of Delamination Variables $\quad \ldots \ldots \ldots . .90$

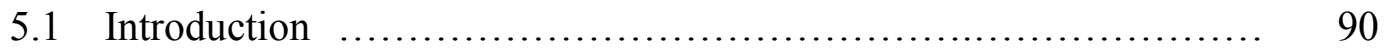

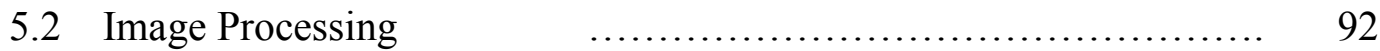

5.3 Statistical Analysis of Void Content $\quad$.......................... 94

5.4 Conclusion $\quad$........................................... 103

Chapter 6: Modelling in Abaqus $\quad$..................... 104

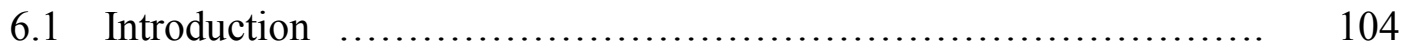

6.2 Simulation Models $\quad$......................................... 106

6.2.1 Model case $1 \quad$................................... 107 
6.2.2 Model case 2

6.2.3 Model case 3

6.2.4 Model case 4

Density maps

6.3 Summary of FEA Models

6.4 Conclusion

Chapter 7: Summary

Concluding Remarks

Future Work

\section{References}

\section{Appendices}

Appendix B: Material: DA 409U/G35 150 Unidirectional Carbon/Epoxy

Appendix C: CLT calculations of Laminate Constants 


\section{List of Illustrations}

Figure 1.1 Composite Materials Applications in Commercial Aircraft $\ldots . .2$

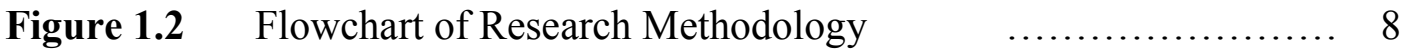

Figure 2.1 $\quad$ (a) 1/1 Plain weave (b) 1/2 Twill weave (c) 1/4 Satin weave ..... 13

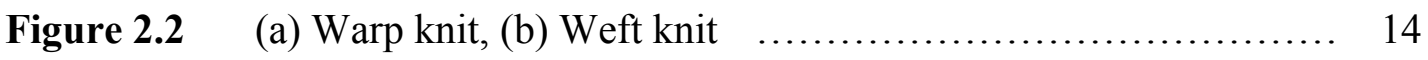

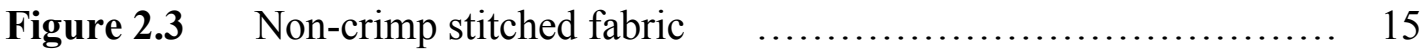

Figure 2.4 Spacer fabric manufactured in single weaving step $\quad \ldots \ldots \quad 16$

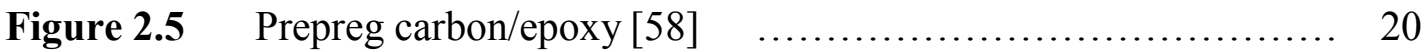

Figure 2.6 Eight fabricated coupons $\quad \ldots \ldots \ldots \ldots \ldots \ldots \ldots \ldots \ldots, 22$

Figure 2.7 Dimensions of testing coupons $(\mathrm{mm}) \quad \ldots \ldots \ldots \ldots \ldots \ldots \ldots .23$

Figure 2.8 Travelling microscope set up to observe the external aspects of the

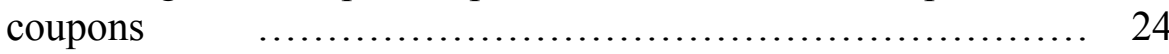

Figure 2.9 Delamination observed near the edge of coupon $6 \quad \ldots \ldots \ldots \ldots .25$

Figure 2.10 Cutting defects at the notch of coupon $7 \quad \ldots \ldots \ldots \ldots \ldots \ldots . . \ldots 25$

Figure 2.11 Observed surface defect in coupon $4 \quad \ldots \ldots \ldots \ldots \ldots \ldots \ldots$

Figure 2.12 Engineering constants obtained from CLT calculations $\ldots \ldots 28$

Figure 2.13 Engineering constants obtained from eLaminate $\mathbb{C} \quad \ldots \ldots \ldots \ldots .28$

Figure 2.14 Extensometer mounted on a composite coupon during a static tensile

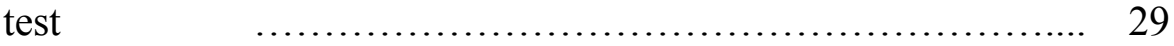

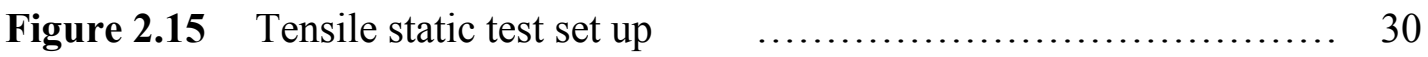

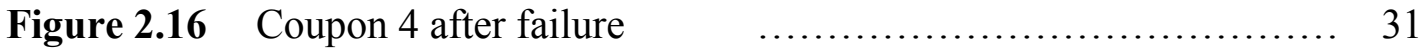

Figure 2.17 Stress-strain data recorded during the tensile static test $\quad \ldots . . \quad 32$

Figure 2.18 Typical cyclic loading parameters $\quad \ldots \ldots \ldots \ldots \ldots \ldots \ldots, 32$

Figure 2.19 Ultra-high resolution X-ray microCT, Carleton University $\quad \ldots . . . . \quad 35$ 
Figure 2.20 Ultra high-resolution X-ray raw image $\quad \ldots \ldots \ldots \ldots \ldots \ldots \ldots . \ldots \ldots$

Figure 3.1 Schematic diagram of application of X-ray $\quad \ldots \ldots \ldots \ldots . . .41$

Figure 3.2 Published fatigue curves of $\pm 45^{\circ}$ and $0 / 90^{\circ}$ laminates $\quad \ldots . . \quad 44$

Figure 3.3 SEM image of sample edge showing delamination $\quad \ldots . .45$

Figure 3.4 SEM images comparing the cut edge (a) to a cross-section (b) of a sample

Figure 3.5 3D volume rendered VOI of CFRP subjected to 400,000 fatigue cycles

Figure 3.6 Virtual slices across CFRP subjected to 400,000 fatigue cycles 46

Figure 3.7 3D volume rendered image of CFRP subjected to 800,000 fatigue cycles

Figure 3.8 3D volume rendered image of CFRP subjected to $1.2 \times 10^{6}$ fatigue cycles

Figure 3.9 Separate damaged areas (a) and damaged material (b) of CFRP sample subjected to $1.2 \times 10^{6}$ fatigue cycles

Figure 3.10 3D volume rendered image of CFRP subjected to $5.5 \times 10^{6}$ fatigue cycles 50

Figure 3.11 Virtual slices through 25\% thickness of CFRP subjected to 5.5 $\mathrm{x} 10^{6}$ cycles

Figure 3.12 Virtual slices through 50\% thickness of CFRP subjected to 5.5 $\mathrm{x} 10^{6}$ cycles

Figure 3.13 Virtual slices through 75\% thickness of CFRP subjected to 5.5 $\mathrm{x} 10^{6}$ cycles

Figure 3.14 DataViewer $58 \mathrm{~mm}$ x $58 \mathrm{~mm}$ virtual slice through thickness 56

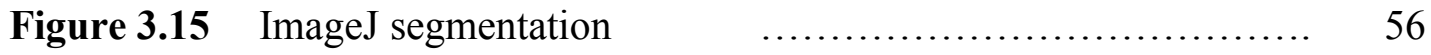

Figure 3.16 3D segmented volume render - ScanIP software $\quad \ldots \ldots \ldots \ldots . \quad 56$

Figure 3.17 3D representation of cracks plotted in MATLAB $\quad \ldots \ldots \ldots \ldots . . . .57$

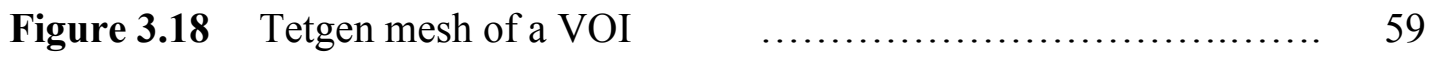

Figure 3.19 Tetrahedral/hexahedral mesh using ScanIP software $\quad \ldots . . \quad 59$ 
Figure 3.20 A tetrahedral mesh of a VOI using ScanIP software $\quad \ldots . .60$

Figure 3.21 A triangular mesh of a VOI using ScanIP software $\quad \ldots . .60$

Figure 3.22 Simulation of $2 \mathrm{D}$ static crack obtained from

Figure 3.23 Arbitrary crack growth simulated using Abaqus $\quad \ldots \ldots \ldots \ldots .61$

Figure 3.24 Flowchart demonstrating proposed tools for exporting microCT scans to Abaqus and their employment modes

Figure 4. 1 RVE of a composite subjected to (a) axial tensile load and b) transverse tensile load

Figure 4. 2 Damage modes; opening, in-plane shear and out-of-plane shear 67

Figure 4. 3 Ply layer thickness under microscope $\quad \ldots \ldots \ldots \ldots \ldots \ldots . . \ldots 8$

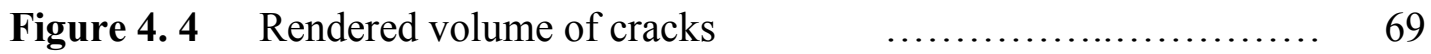

Figure 4.5 Virtual cross-section of the sample in X-Y (transverse) plane clearly identifying the $0 / 90$ layers

Figure 4. 6 Virtual cross-section image of X-Z plane (a), minimum threshold method (b) and triangle threshold method (c)

Figure 4. 7 Minimum auto threshold levels for stages of fatigue life in the $X-Z$ plane

Figure 4. 8 Virtual cross-section of X-Y plane (a), minimum threshold method (b) and triangle threshold method (c)

Figure 4.9 Virtual cross-section of Y-Z plane (a), minimum threshold method (b) and triangle threshold method (c)

Figure 4. 10 Threshold levels applied by auto 'minimum' method for the (a) Y-Z and the (b) X-Y planes and auto 'triangle' method for the (c) Y-Z and the (d) X-Y planes

Figure 4. 11 Suspected fibre breakage detected in virtual X-Z cross-section 75

Figure 4. 12 Fatigue crack growth of mode I opening cracks $\quad \ldots \ldots 77$

Figure 4. 13 Fatigue crack growth of mode I closing cracks $\quad \ldots \ldots 77$ 
Figure 4. 14 Figure 4.14: Crack displacements of mode I opening cracks $\quad 78$

Figure 4. 15 Crack displacements of mode I closing cracks $\quad \ldots \ldots \ldots \ldots \ldots . . .68$

Figure 4. 16 Fatigue crack growth of mode II opening cracks $\quad \ldots . . \quad 81$

Figure 4. 17 Fatigue crack growth of mode II closing cracks $\quad \ldots . . \quad 81$

Figure 4. 18 Crack displacements of mode II opening cracks $\quad \ldots \ldots \ldots \ldots . . . \quad 82$

Figure 4. 19 Crack displacements of mode II closing cracks $\quad \ldots \ldots \ldots \ldots . . . \quad 82$

Figure 4. 20 Fatigue crack growth of mode III opening cracks $\quad \ldots \ldots \ldots \ldots . . . . \quad 84$

Figure 4. 21 Fatigue crack growth of mode III closing cracks $\quad \ldots \ldots \ldots \ldots . . . . \quad 84$

Figure 4. 22 Crack displacements of mode III opening cracks $\quad \ldots \ldots \ldots \ldots . . . . \quad 85$

Figure 4. 23 Crack displacements of mode III closing cracks $\quad \ldots \ldots \ldots \ldots \ldots \quad 85$

Figure 4. 24 General trend of fatigue crack growth rate in the 3 orthogonal

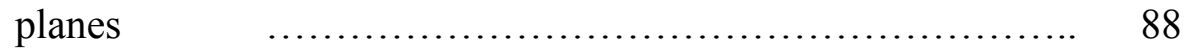

Figure 4. 25 General trend of crack displacements in the 3 orthogonal planes 88

Figure 5.1 Void content as a \% of area throughout fatigue life $\quad \ldots \quad 93$

Figure 5.2 Accumulated cracks in Y-Z plane, pristine state $\quad \ldots . \ldots \ldots \ldots . . .65$

Figure 5.3 Accumulated cracks in X-Y plane, pristine state $\quad \ldots \ldots \ldots \ldots . . . .65$

Figure 5.4 Accumulated cracks in Y-Z plane, 200,000 cycles $\quad \ldots \quad 96$

Figure 5.5 Accumulated cracks in X-Y plane, 200,000 cycles $\quad \ldots \quad 96$

Figure 5.6 Accumulated cracks in Y-Z plane, 400,000 cycles $\quad \ldots \quad 97$

Figure 5.7 Accumulated cracks in X-Y plane, 400,000 cycles $\quad \ldots \quad 97$

Figure 5.8 Accumulated cracks in Y-Z plane, 600,000 cycles $\quad \ldots \quad 98$

Figure 5.9 Accumulated cracks in X-Y plane, 600,000 cycles $\quad \ldots \quad 98$

Figure 5.10 Accumulated cracks in Y-Z plane, 800,000 cycles $\quad \ldots \quad 99$

Figure 5.11 Accumulated cracks in X-Y plane, 800,000 cycles $\quad \ldots . \quad 99$ 
Figure 5.12 4 cracks spotted in the $Y-Z$ plane (upper left), and the same cracks were traced in the $X-Y$ plane (under), with their through-thickness position defined for each. (Upper right) A 3D reconstructed rendered volume of the VOI under investigation

Figure 6.1 Asymmetric boundary conditions on $\mathrm{X}$ and $\mathrm{Y}$ axes of quarter sample

Figure 6.2 Biased seeding of 3D Abaqus model 107

Figure 6.3 3D Abaqus model mesh 107

Figure 6.4 Abaqus composite lay-up 107

Figure 6.5 Principle stress in Case 1 107

Figure 6.6 Principle stress contours of the segmented model case 1 109

Figure 6.7 A predefined crack at mid-notch 110

Figure 6.8 Von Mises stress contours of stationary crack 110

Figure 6.9 3 Contours of $\mathrm{K}_{\mathrm{s}}$ versus time at the crack set of nodes

Figure 6.10 Crack location

Figure 6.11 Von Mises stress contours of growing crack

Figure 6.12 Crack before loading (left) and after loading (right)

Figure 6.13 Level set $\boldsymbol{\psi}$ for crack

Figure 6.14 Level set $\varphi$ for crack

Figure 6.15 VOI of 59 images imported from microCT scans

Figure 6.16 Rendered volumes of successive intervals of fatigue life

Figure 6.17 Cracks mask of the VOI imported from microCT

Figure 6.18 Matrix mask of the VOI imported from microCT

Figure 6.19 Matrix quadratic tetrahedral mesh of VOI

Figure 6.20 Virtual cut in the mesh to identify cracks 
Figure 6.21 Cavities traced in virtual slices of solid mesh $\quad \ldots \ldots \ldots \ldots \ldots, 118$

Figure 6.22 Plots of density over fatigue life for 3 material sections $\quad \ldots \ldots \quad 119$

Figure 6.23 Plots of density contours on virtual slices of meshed volumes 120

Figure 6.24 Orphan mesh of VOI imported into Abaqus excluding voids mask 121

Figure 6.25 (a) Cracks locations and (b) boundary conditions on orphan mesh

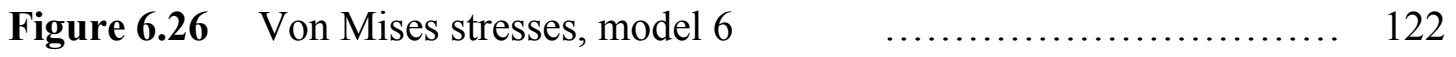

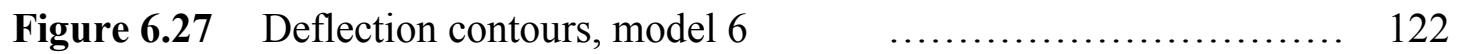

Figure 6.28 Cross-section of deformed model 6 across $\mathrm{Y}$-axis (left) and $\mathrm{X}$-axis (right)

Figure 6.29 Plots of deflection contours, partition of model $6 \quad \ldots \ldots \ldots \ldots . . .6123$ 


\section{List of Tables}

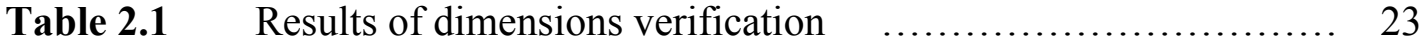

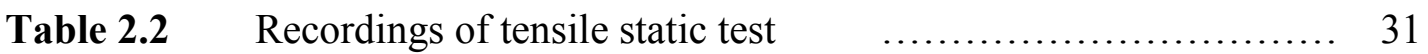

Table 2.3 Properties of normalized main spectra $\quad \ldots \ldots \ldots \ldots \ldots \ldots \ldots, 33$

Table 2.4 Properties of normalized main spectra per flight phase $\quad \ldots \ldots \quad 33$

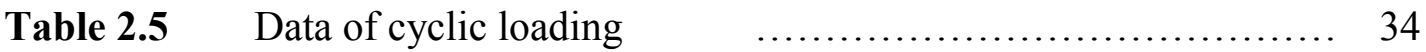

Table 2.6 Actual applied loads on fatigue testing frame $\quad \ldots \ldots \ldots \ldots \ldots . . . .34$

Table 4.1 Cracks coordinates and change in length for X-Z plane $\quad \ldots . .79$

Table 4.2 Cracks coordinates and change in length for $\mathrm{Y}-\mathrm{Z}$ plane $\quad \ldots \ldots \quad 80$

Table 4.3 Cracks coordinates and change in length for X-Y plane $\quad \ldots \ldots \quad 83$

Table 4.4 Summary of quantitative analysis $\quad \ldots \ldots \ldots \ldots \ldots \ldots \ldots \ldots \ldots \ldots \ldots \ldots \ldots$

Table 4.5 Comparison of published and experimental data $\quad$............. 87

Table 5.1 Change of crack length in sample with number of fatigue cycles 100

Table 5.2 Progression of crack length in the in-plane shear plane $\quad \ldots \ldots \quad 101$

Table 5.3 Progression of crack length in the out-of-plane shear plane $\ldots \ldots \quad 101$

Table 6.1 Calculation of stress concentration factor, $\boldsymbol{K}_{t} \quad \ldots \ldots \ldots \ldots \ldots$

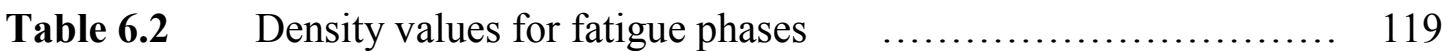

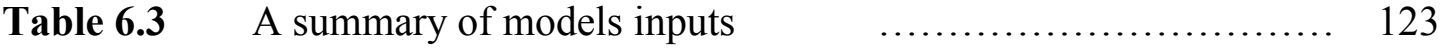

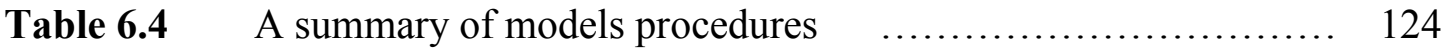




\section{List of Appendices}

Appendix A Test Sample Specifications $\quad$........................... 142

Appendix B Material: DA 409U/G35 150 Unidirectional Carbon/Epoxy Prepreg

Appendix C CLT calculations of Laminate Constants $\quad$............... 144

Appendix D MATLAB Code for 3D Plotting of MicroCT scans ........ 


\section{Nomenclature}

\section{List of Symbols}

Latin
a Crack length
$\boldsymbol{C}_{\boldsymbol{I}} \quad$ Constant (normal mode)
$\boldsymbol{C}_{\boldsymbol{I I}} \quad$ Constant (in-plane shear mode)
$\boldsymbol{C}_{\text {III }}$ Constant (out-of-plane shear mode)
$\boldsymbol{E}_{\boldsymbol{L}} \quad$ Longitudinal modulus
$\boldsymbol{E}_{\boldsymbol{T}} \quad$ Transverse modulus
$\boldsymbol{E}_{1}, \boldsymbol{E}_{2} \quad$ Elastic Constants
$\boldsymbol{G}_{\boldsymbol{L} T} \quad$ Shear modulus
$\boldsymbol{G}_{\mathrm{n}} \quad$ Fracture energy (normal mode)
$\boldsymbol{G}_{\boldsymbol{s}} \quad$ Fracture energy (in-plane shear mode)
$\boldsymbol{G}_{\boldsymbol{t}} \quad$ Fracture energy (out-of-plane shear mode)
$\boldsymbol{G}_{\boldsymbol{I C}}$ Critical Fracture Toughness (Opening Mode I)
$\boldsymbol{G}_{\text {IIC }}$ Critical Fracture Toughness (In-plane Shear Mode II)
$\boldsymbol{G}_{\text {IIIC }}$ Critical Fracture Toughness (Out-of-Plane Shear Mode III)
$\boldsymbol{h} \quad$ Laminate thickness
I Moment of inertia
$\boldsymbol{K}_{\boldsymbol{t}} \quad$ Theoretical Orthotropic Stress Concentration Factor
$\boldsymbol{K}_{t \infty} \quad$ Stress Concentration Factor for an Infinite Orthotropic Continuum
$\boldsymbol{m}_{\boldsymbol{I}} \quad$ Constant (normal mode)
$\boldsymbol{m}_{\boldsymbol{I I}} \quad$ Constant (in-plane shear mode)
$\boldsymbol{m}_{\text {III }} \quad$ Constant (out-of-plane shear mode)
$N \quad$ Number of cycles
$\boldsymbol{Q}_{i j} \quad$ On-axis stiffness terms
$P \quad$ Load
$\boldsymbol{R} \quad$ Stress Ratio
$S_{i j} \quad$ On-axis compliance terms
$\boldsymbol{S}_{\boldsymbol{n}} \quad$ Normal strength
$\boldsymbol{S}_{\boldsymbol{s}} \quad$ In-plane shear strength
$\boldsymbol{S}_{t} \quad$ Out-of-plane shear strength
$\boldsymbol{t} \quad$ Threshold

$\begin{array}{cll}\text { Greek } & \alpha & \text { Finite width correction factor } \\ & \sigma_{\max } & \text { Maximum principle stress } \\ \boldsymbol{\sigma}_{\text {nom }} & \text { Nominal stress } \\ \boldsymbol{\mu} & \text { Micron } \\ \boldsymbol{\rho} & \text { Density } \\ \boldsymbol{\nu} & \text { Poisson's ratio } \\ \boldsymbol{\psi} & \text { level set of crack tip } \\ \boldsymbol{\varphi} & \text { level set of crack surface }\end{array}$




\section{List of Acronyms}

$\begin{array}{ll}\text { AE } & \text { Acoustic Emmisions } \\ \text { AU } & \text { Acousto-Ultrasonics } \\ \text { CFRP } & \text { Carbon Fibre-Reinforced Polymer } \\ \text { CLT } & \text { Classical Laminate (Plate) Theory } \\ \text { CT } & \text { Computed Tomography } \\ \text { DCB } & \text { Double Cantilever Beam } \\ \text { DIC } & \text { Digital Image Correlation } \\ \text { DT } & \text { Destructive Testing } \\ \text { ENF } & \text { End Notch Flexure } \\ \text { FALSTAFF } & \text { Fighter Aircraft Loading Standard For Fatigue } \\ \text { FEA } & \text { Finite Element Analysis } \\ \text { LEFM } & \text { Linear Elastic Fracture Mechanics } \\ \text { MMB } & \text { Mixed-Mode Bending } \\ \text { MMELS } & \text { Mixed-Mode End-Loaded Split } \\ \text { MTS } & \text { Material Test System } \\ \text { NCS } & \text { Non-Crimp Stitched } \\ \text { NDT } & \text { Non-destructive Testing } \\ \text { PAN } & \text { Polyacrylonitrile } \\ \text { PE } & \text { Polyethylene } \\ \text { PET } & \text { Polyethylene terephthalate } \\ \text { PMC } & \text { Polymer Matrix Composites } \\ \text { PP } & \text { Polypropylene } \\ \text { RUC } & \text { Repeat Unit Cell } \\ \text { RVE } & \text { Representative Volume Element } \\ \text { SCF } & \text { Stress Concentration Factor } \\ \text { SEM } & \text { Scanning Electron Microscope } \\ \text { SIF } & \text { Stress Intensity Factor } \\ \text { SRCT } & \text { Synchrotron Radiation Computed Tomography } \\ \text { TWIST } & \text { Transport Wing Standard } \\ \text { UT } & \text { Ultrasonic Technique } \\ \text { VCCT } & \text { Virtual Crack Closure Technique } \\ \text { VOI } & \text { Volume of Interest } \\ \text { WWFE } & \text { World-Wide Failure Exercise } \\ & \end{array}$




\section{Chapter 1:}

\section{Introduction}

\subsection{Background}

In the late 1950s, carbon fibres were developed in Britain and since then, have been used as reinforcements for polymer matrix composites (PMC) mainly for military applications. The carbon fibre industry faced varying fortunes over time, with reduced activity from the late 1960s to late 1970s, and again in the 1990s after the end of the Cold War. By 2002, the economic climate for producing carbon fibres was more favourable, prices decreased and market experienced growing demand for lightweight materials of high stiffness and strength for manufacturing composites used in expanding commercial segments such as commercial aerospace, sporting goods, reinforcements for structures and buildings in earthquake zones, reinforced fuel tanks and wind turbines [1].

Particularly in the aircraft industry, where high stiffness-to-weight is a crucial demand, the last two decades have seen a steep rise in the use of advanced laminated carbon fibre 
reinforced polymer (CFRP) composites to replace metal parts with the objectives of improving fuel efficiency, reducing airframe weight and decreasing total part numbers. Contribution of composite structures to the overall body weight is around $25 \%$ in some aircraft applications such as the Airbus A380, whereas the Boeing 787 Dreamliner and A350 are composed of 50\% composites by weight and the aluminum structures in the wings and fuselage are entirely replaced by composites [2], as shown in Figure 1.1. Nowadays, the largest design and manufacturing entities continue to integrate composite parts into new aircraft at a rapid pace, at the same time aircraft composite parts are under increasing consideration from the civil aviation authorities.

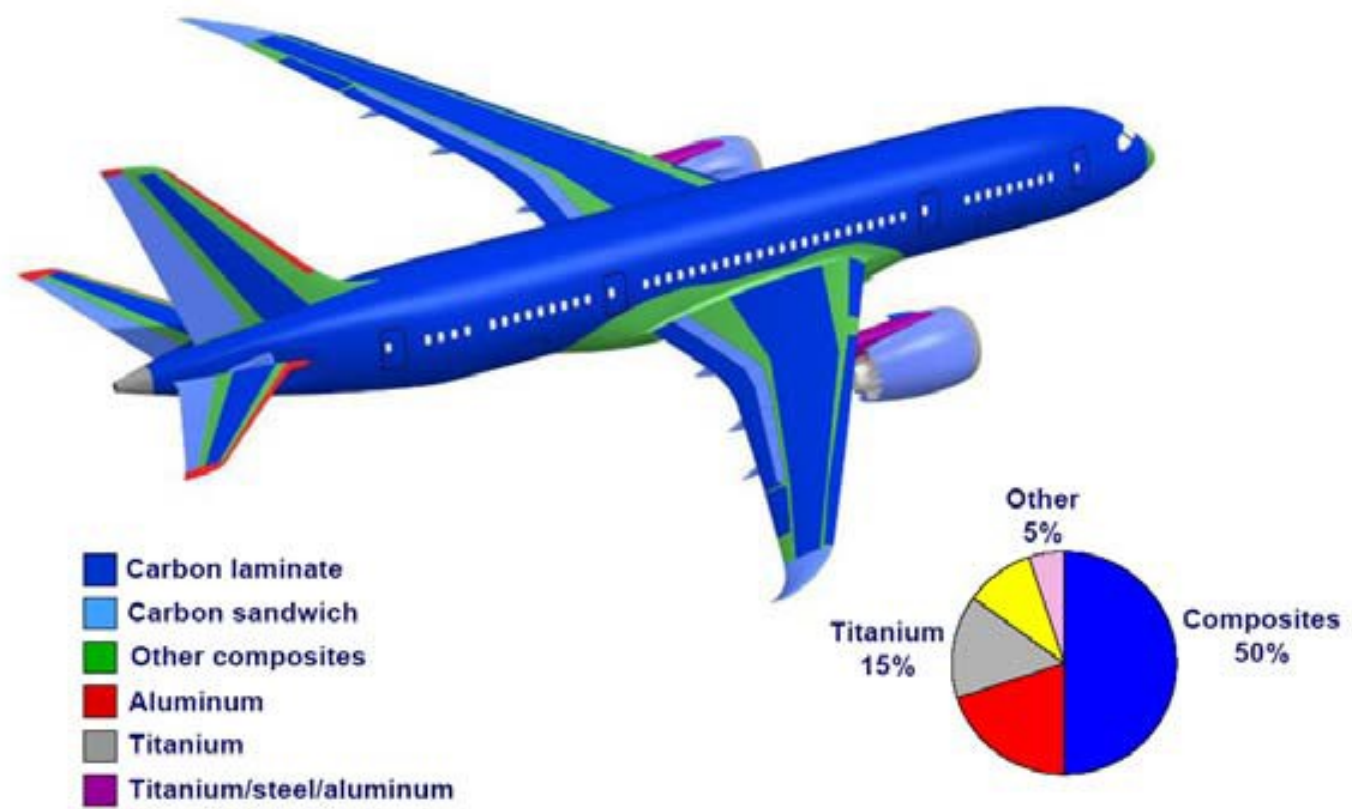

Figure 1.1: Composite Materials Applications in Commercial Aircraft [2]

Considering that textiles made of carbon fibres and other available organic and inorganic fibres are primarily manufactured for their technical and performance properties, their geometrical structure had been thoroughly studied over years to develop models that are capable of predicting their mechanical behaviour under various loading conditions. These models will be discussed herein. 


\subsection{Rationale}

Quality standards and damage tolerance analysis techniques are unique for each industry; within the aircraft industry there is an overarching need to maintain flight safety over the entire life of the aircraft and under a wide range of operating conditions. In order to achieve this objective, fatigue damage in parts experiencing constant or variable amplitude loading are required to lie within damage tolerance limits for composites [3, 4], requiring accurate damage prediction methods during design.

Typically characterized by their orthotropic and heterogeneous nature, prediction of evolution and progression of damage in multidirectional laminated composites becomes a complicated task. The Third World-Wide Failure Exercise (WWFE-III) [5] was concluded in 2011; aimed at establishing a benchmark for damage, fracture and continuum mechanics theories for fibre composites and to reduce the gap between academia and industry in terms of reliability. Professor Hashin [6], whose theories are widely implemented for predicting failure in laminated reinforcement composites, declined to participate in WWFE-I with the following reason (quoting from his letter to the WWFE-I organizers) [5]:

".. my only work in this subject relates to failure criteria of unidirectional fibre composites, not to laminates ............ I must say to you that I personally do not know how to predict the failure of a laminate (and furthermore, that I do not believe that anybody else does)".

Unfortunately, the above statement was true to a great extent. Results of the later WWFEII and III were associated with uncertainty and failure theories that had significant variability in failure prediction accuracy $[5,7]$. Continuum models for defining failure criteria in textile composites are available in literature and are widely implemented for 
simulating failure scenarios using Finite Element Analysis (FEA) and analytical design software [8 - 14]. However, these criteria can only predict the final event of damage at the macroscopic scale and have a limited capability for predicting the preceding progression scenario until failure, damage mechanisms and defining the criteria leading to the early evolution of cracks at the microscopic scale [15].

To compensate for the lack of understanding of the damage evolution mechanisms in PMCs, aircraft designers have adopted a conservative design approach relying heavily on experimental testing, load enhancement factors and over-designed components. While the resulting structures meet or exceed safety requirements, they may be overweight and structurally inefficient. In order to support the development of improved damage models for the aerospace industry, the phenomenological experimental and numerical study described in this thesis was carried out to examine the effect of cyclic loading on the evolution of microscopic damage in unidirectional laminae.

\subsection{Thesis Objectives}

This research focused on cyclic fatigue loads generally experienced by typical modern aircraft structures during flight. The initiation-to-failure process of fatigue damage in composite laminates was explored by utilizing a systematic approach using data collected directly from composite samples subjected to constant amplitude loading and examined non-destructively. FEA is implemented for simulating damage propagation of various case studies. However, simulations using available FEA tools have limited ability of combining multi-mode damage mechanisms; delamination, splitting, ply crack and fibre rupture. Hence, different simulation methods were employed within the FEA framework to address 
several cases. Definite cracks were simulated traced and compared to experimental findings.

Experimental work was carried out employing state-of-the-art non-destructive inspection with newly developed methods for performing analytical investigations to produce quantitative information on internal structures. To improve the robustness and efficiency of PMC aircraft structures as the overall goal, this research is aimed at answering the following research questions through a combined experimental and analytical approach:

- Is it possible to use the X-ray microCT tool to qualitatively identify the progressive sequence of damage mechanisms, namely delamination, interfacial debonding (matrix cracks), interfacial slippage and fibre breakage?

- What algorithms are needed to incorporate microCT scan data into Abaqus?

- What are the fatigue crack growth rates in the 3 orthogonal planes of a composite laminate and what are their strain energy release rate according to Linear Elastic Fracture Mechanics (LEFM) laws as measured non-destructively?

- Contrary to literature reviews assuming a similar behaviour of traction-separation response for both in-plane and out-of-plane shear modes (e.g. mode II and mode III), is it possible to capture the crack displacements in either modes separately?

\subsection{Thesis Contributions}

The core contribution of this work is the establishment of a methodology to quantify damage in polymer matrix composites to support damage modelling based on linear elastic fracture mechanics simulations. This topic was the subject of a conference paper presented 
by the author in SAMPE 2015 [16], as well as a more comprehensive journal paper addressing the quantitative analysis of genuine damage cracks. The paper was accepted in a peer-reviewed journal and appeared online in October 2015 [17]. Architecture of textile reinforcements used for composites occupied the introductory part of research through a study of geometrical modelling techniques at the meso-scale. A review article discussing this topic was published in a peer-reviewed journal in October 2014 [18].

For studying micro-damage propagation and geometrical configurations, a state-of-the-art high resolution XR-microcomputed tomography (XR microCT) instrument recently acquired at Carleton University was used. Novel experimental procedures and preliminary results were developed and presented in two reviewed conference papers. Qualitative assessment of damage propagation in CFRP subjected to cyclic fatigue loading was presented in SAMPE 2014 [19], whereas a procedure for importing microCT scans into Abaqus tool was proposed and described in CAMX 2014 [20]. A summary of papers published and presented are listed hereunder.

- ElAgamy N and Laliberté J (2015). Verification of parameters for modelling of fatigue propagation in laminated CFRP Composites. In: SAMPE conference (Society for Advanced Materials and Process Engineering), Baltimore MD, USA, May 18-21 [16].

- ElAgamy N, Laliberté J and Gaidies F (2015). Quantitative Analysis of fatigue cracks in laminated CFRP composites using micro-computed tomography. Journal of Composite Materials. First published online on October $1^{\text {st }}, 2015$.

DOI: $10.1177 / 0021998315608252$. 
- ElAgamy N and Laliberté J (2014). Historical development of geometrical modelling of textiles reinforcements for polymer composites: a review. Journal of Industrial Textiles. First published on October 20, 2014. DOI: 10.1177/1528083714555781 [18].

- ElAgamy N, Laliberté J, Gaidies F and Goldak J. (2014). Qualitative characterization of fatigue damage propagation in laminated carbon fibre reinforced polymers by using micro-computed tomography. In: SAMPE conference (Society for Advanced Materials and Process Engineering), Seattle WA, USA, June 2-6 [19].

- ElAgamy N, Martin C, Sachdeva T and Laliberté J (2014). Proposed procedure to simulate 3D multi-mode fatigue behaviour in laminated CFRP using micro-CT scans in ABAQUS. In: CAMX conference (The Composites and Advanced Materials Expo), Orlando FL, USA, October 12-16 [20].

This research received "Carleton University Development Grant" in 2014; awarded for the project entitled: "Development and Validation of a Physical-based Methodology to Predict Progressive Damage Development in Advanced Polymer Composite Materials”.

\subsection{Research Methodology}

Work began with a study of the structures of available textiles reinforcements for selecting material for subsequent investigations. Laminated cross-ply configuration was selected and the supplied material was analytically and experimentally studied. Out of eight coupons, one coupon was subjected to consecutive fatigue testing which was interrupted at intervals to perform microCT scanning then perform quantitative analysis. In a parallel procedure, four samples of different material were tested and scanned, each at a different fatigue interval for providing a qualitative assessment of fatigue progression. A procedure was implemented for importing microCT scans in Abaqus using these qualitative scans. 
Analyzing void content combined with LEFM laws provided information on each of the fracture modes independently. Genuine pre-existing voids were imported and simulated in Abaqus for comparing with experimental results. Simulation models were performed to define limitations in modelling. A flowchart highlighting main steps is seen in Figure 1.2.

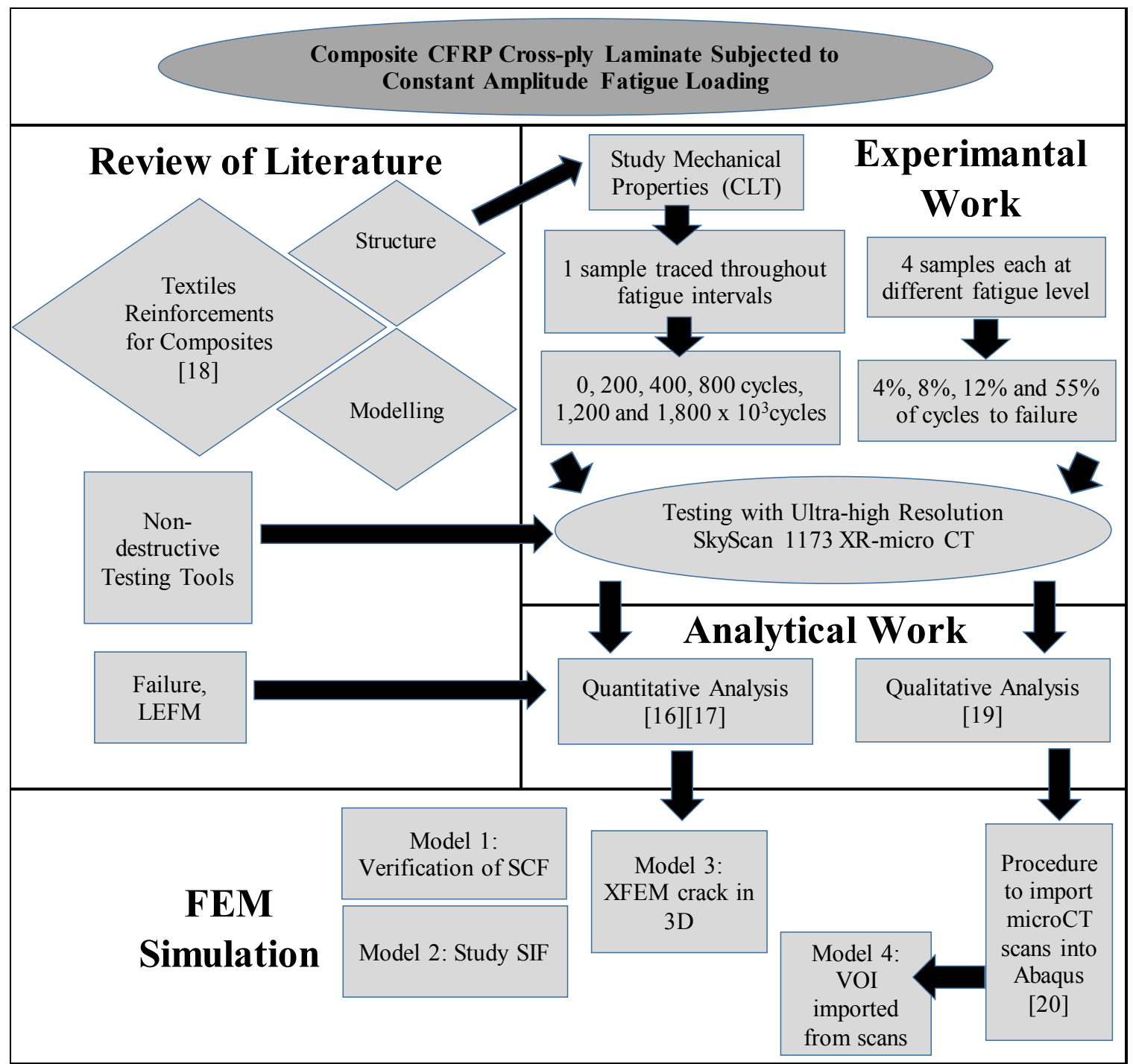

Figure 1.2: Flowchart of Research Methodology

\subsection{Thesis Organization}

The structure of this thesis dissertation is divided over 7 chapters, organized as follows:

Chapter 2 presents a review of literature discussing the conventional and recently developed geometrical structures of technical textiles used for polymer reinforcements. 
Further, geometrical modelling techniques that are available in literature are presented and discussed. Based on this review, the structural configuration of material used for this work was selected, and its physical and mechanical specifications were investigated which led to estimating the material's theoretical properties; presented herein. Fabrication of testing coupons and quality inspection procedures are also detailed.

Methodology is introduced in Chapter 2 detailing both mechanical testing and description of the non-destructive inspection technology (x-ray micro computed tomography). Tensile static and fatigue testing are detailed in terms of testing parameters, machine set-up and preliminary results. Scanning parameters and set-up associated with XR-microCT method are detailed.

A qualitative assessment of the probed material using XR-microCT is presented in Chapter 3. This chapter begins with a review of available non-destructive testing methods and their areas of implementation, with a focus on the XR-microCT technique in terms of theory, applications and published results. Then, an analysis of the obtained scans was introduced from a qualitative perspective, by studying the volume rendered images of each sample, hence concluding a hysteresis of interacting modes of damage. A procedure for simulating $\mathrm{XR}$ microCT scans in commercial FEA tool, Abaqus, is proposed and demonstrated. The procedure involved virtual slicing, segmentation, 3D plotting, meshing and simulation.

In Chapter 4, a quantitative analysis was carried out on damage in terms of crack counts, their statistical distribution and progressive length with no destructive processes associated with the experimentation by using the high resolution microCT scanning. This allowed for 
tracking genuine pre-existing cracks rather than artificially embedded cracks commonly imposed within experimental procedures.

Further implementation of theoretical aspects are presented in Chapter 5. A thorough investigation was carried out, where 4 distinct cracks were traced starting their pristine state and forward throughout their fatigue life in both virtual Y-Z and X-Y planes, relative to in-plane and out-of-plane shear modes. This allows for extracting the data necessary for defining the crack growth rate, and displacements relative to the onset of both shear modes. Hence the traction-separation response in both in-plane and out-of-plane shear modes could be distinguished; unlike the conventional assumption of similar response for both modes.

Chapter 6 introduced four simulation model cases for assessing capabilities and limitations of available FEA software. Case 1 was created to verify theoretical data of stress concentration factors of a notched specimen, whereas case 2 presented a study on the stress intensity factors. An extended finite element method crack type was presented in case 3 . Finally in case 4, a genuine X-ray microCT scanned object was imported into Abaqus following the procedure detailed in Chapter 3.

Chapter 7 presented a discussion of the thesis outcome, overall concluding remarks and suggested future work. 


\section{Chapter 2:}

\section{Experimental Methodology}

\subsection{Introduction}

Textiles used as reinforcements for composite materials broadly vary in their geometrical structures and physical properties. Therefore part designers consider thorough simulation procedures in order to predict their future mechanical response and adapt their end-use accordingly. It is a question of both economical aspect and safety measures.

In this chapter, a review of literature was conducted to study conventional and recently developed textile geometrical structures used for polymer reinforcements and their available modelling techniques. A more detailed article discussing this topic was published in a peer-reviewed journal [18]. The objective of this review was to present all available composite reinforcement structures and to highlight difficulties in their geometrical modelling despite their superior potential performance, so as to justify selecting the structural configuration of the material used. Mechanical specifications of the investigated material were discussed, and a detailed testing plan was designed. 


\subsection{Review of Literature}

Mechanical behaviour of fibre reinforced polymer composites is governed primarily by the geometrical structure of the textile reinforcements. Consistent permeability, predictable impregnation and even distribution of resin are key factors in reaching targeted mechanical performance, whereas these parameters and others are functions of the geometrical composition. Hence numerous analytical geometrical models have been described in literature covering most of the conventional commonly used weave reinforcements. This section aimed to trace the physical concepts used for modelling the geometrical structures of textile reinforcements at the meso-scale and compare applicability of various analytical and numerical models to types and geometrical dimensionality of woven textile structures.

\subsubsection{Geometrical Structure of Technical Textiles}

Structures of textiles used as reinforcements for polymer composites are hierarchical in nature $[21,22]$. They can be described in terms of dimensional scale involving 5 length scales : (1) nanoscopic scale; an intermediate size between microscopic and molecular structures; referring to dimensions ranging $1-100 \times 10^{-9} \mathrm{~m},(2)$ microscopic scale, relating to individual fibres with diameters around $10 \mu \mathrm{m}$ and represented by repeating unit cells (RUC), (3) mesoscopic scale with dimensions ranging between $0.5 \mathrm{~mm}-10 \mathrm{~mm}$ and characterized by the representative volume element (RVE), (4) macroscopic scale, relating to composite structures with dimensions ranging typically between $0.1 \mathrm{~m}$ and $1.0 \mathrm{~m}$, and finally (5) megascopic scale representing the part with typical dimensions above $1.0 \mathrm{~m}$.

Alternatively, these structures can be described relative to their geometrical dimensionality at the mesoscopic level. Geometrical dimensionality is generally relative to the mode of 
manufacture starting with 1D fibre assemblies and progressing towards more complex 2D, 2.5D and 3D textile structures. This section presented a review of geometrical modelling of reinforcements in terms of their geometrical dimensionality at the mesoscale; unit cell.

Yarns, tows and rovings constitute the common, basic 1D textile form. Yarns are made up of a bundle of twisted staple fibres, whereas a tow is a large strand of continuous fibre filaments devoid of definite twist, collected in loose, rope-like form, usually held together by crimp. In yarn production, a roving is an intermediate state between a sliver and yarn, i.e. a drafted sliver, different from that in composites manufacturing, where a roving is a lightly twisted flat tow [23].

2D textiles are classified as being of limited thickness; a maximum of 2 yarns will be superimposed at any point of a 2D textile. 2D weaves are made of 2 sets of warp and weft yarns interlacing at right angles in one plane. They are produced on a weaving loom where warp yarns go through a lifting mechanism composed of a number of harnesses, and weft yarns are fed at a constant rate through sheds, which are the gaps formed between warp yarns when the harnesses go up or down [24]. The sequence of lifting warp yarns defines the weave pattern [25] expressed in $n / m$, meaning that the warp end passes over ' $n$ ' and under ' $m$ ' number of weft picks, thus the sum $(m+n)$ indicates the number of harnesses needed to create that pattern. Common patterns are the plain weave (1/1), Figure 2.1(a), twill weaves (1/2, 2/2, 2/3 etc.), Figure 2.1(b), and satin weaves (5H or 8H), Figure 2.1(c).

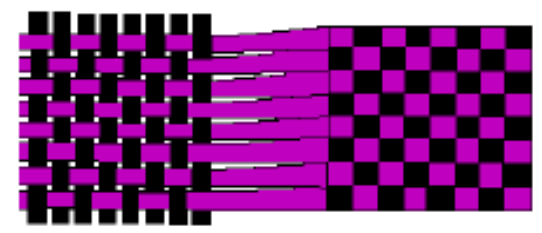

Figure 2.1:

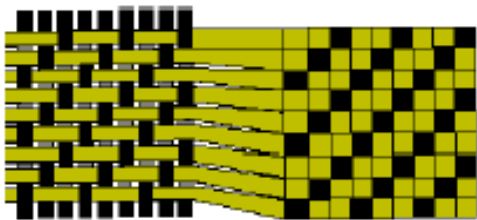

(b) $1 / 2$ Twill weave modified from [24]

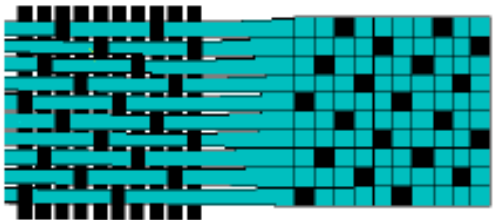

(c) 1/4 Satin weave 
Other forms include braids, knitted and non-woven textiles. 2D braids are analogous to weaves but they are formed as flat or tubular textile structures in diagonal interlacing. 2D knitted fabrics are either warp knitted, Figure 2.2 (a), or weft knitted, Figure 2.2 (b). 2D knitted fabrics are not ideally suitable for technical applications, because yarn paths are not conducive to high fibre volume fractions in the preforms. 2D non-woven textiles are fibreto-fabric products requiring no prerequisite yarn spinning and they are used for insulation. Holding of the fibrous layers, called 'batt', is performed either mechanically by needle felting, chemically with a bonding agent or by thermal bonding [24].

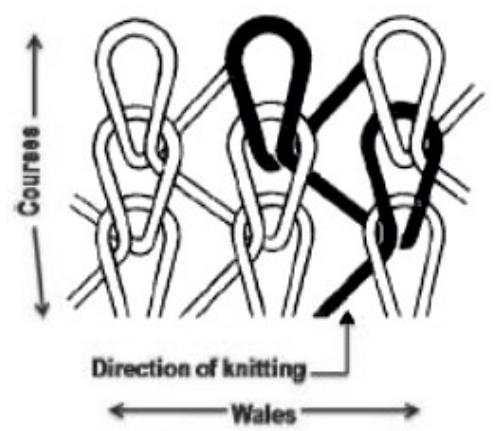

Figure 2.2: (a) Warp knit,

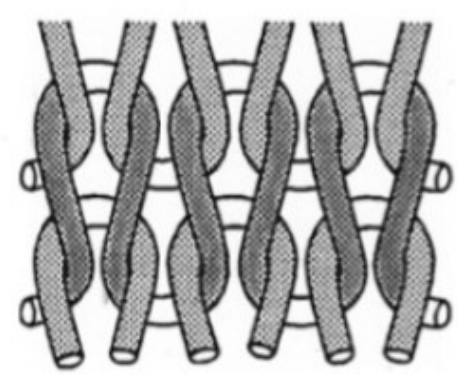

(b) Weft knit, modified from [25]

Textiles that are relatively thick and feature a higher number of superimposed layers made of multiple planes of 2D structures with possibly some light yarns interlaced in the third dimension to bind the structure, but devoid of significant vertical interlacing from different layers are sometimes labeled as $2.5 \mathrm{D}$ textiles. Some nonwovens, non-crimp stitched fabrics (NCS), velvets and interlock weaves can be considered as $2.5 \mathrm{D}$. They generally behave much like 2D textiles with the exception of greater bending rigidity due to their thickness. Carbon reinforcements made of NCSs, Figure 2.3, combine unidirectional crimp-free yarn layers laminated at different angles and assembled by warp-knitting; the layered structure being designed such that the PMC part to be produced can withstand the expected stresses in a specific required direction [24]. Interlock weaves are another form of $2.5 \mathrm{D}$ textiles. In 
2.5D interlock weaves, warp weavers penetrate through planes of warp and weft yarns at an angle of inclination to form the integral structure.

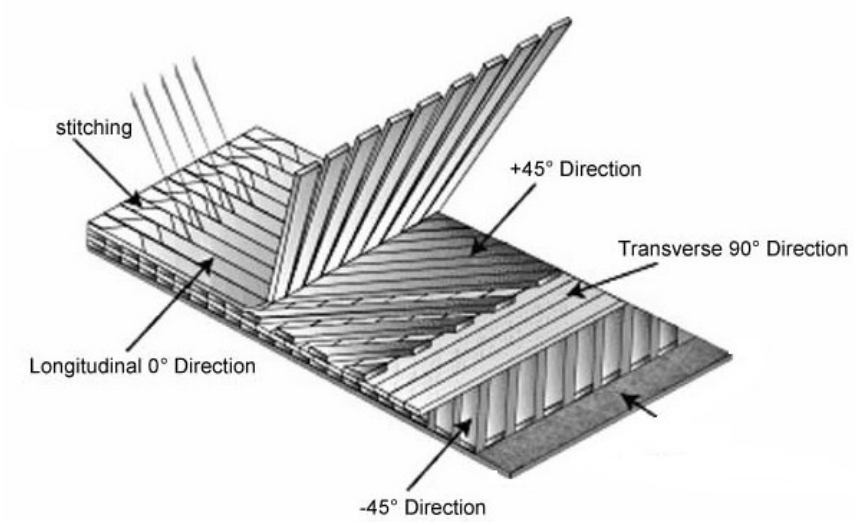

Figure 2.3: Non-crimp stitched fabric, modified from [25]

Generally, 3D textiles are flexible textile products; either planar with a substantial thickness compared to the other 2 planar dimensions, or produced in complex non-planar shapes. 3D reinforcements in the composites industry may optionally replace multilayer laminated 2D reinforcements for providing better through-thickness and inter-laminar properties, higher impact resistance [25], and avoiding the weak resin-rich interfaces commonly present in laminated 2D reinforcements [26].

Examples of 3D textiles are the spacer fabrics, Figure 2.4, which are composed of 2 parallel layers of woven or warp knitted fabrics with z-directional yarns inserted between both layers to bond the structure and create its thickness. They provide good dimensional stability and high stiffness to weight ratios in the composite parts [27]. Particularly for composites production using resin vacuum infusion, Mack [28] recommended 3D spacer fabrics as reinforcing inter-laminar infusion mediums to enhance resin distribution and facilitate its penetration. Another major application of developed forms of 3D warp knitted spacer fabrics is textile reinforced concrete [29]. 


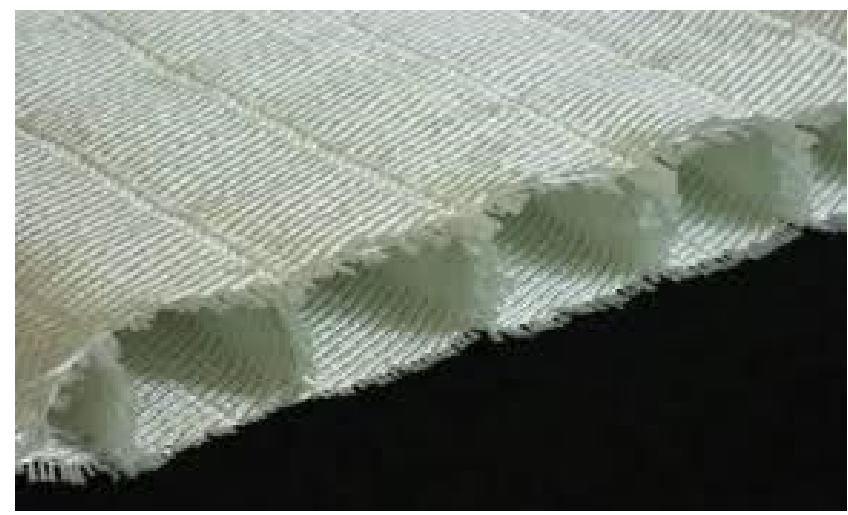

Figure 2.4: Spacer fabric manufactured in single weaving step [27]

As opposed to 2D braids, 3D braided composites combine shear stiffness and strength, reduce or eliminate delamination, and provide high impact resistance, high conformability and high torsional rigidity [30]. One last form of 3D textiles are the crimp-free 3D orthogonal non-woven preforms, which refer to structures of orthogonally non-interlaced yarns that can be produced on 3D weaving machines. The process involves the insertion of pure matrix to fill the areas between fibre bundles, to form composites in a further processing step $[25,31]$.

\subsubsection{Geometrical Modelling of Technical Textiles}

Geometrical modelling of textile structures has been the subject of investigations starting from the early decades of the 20th century. The introduction of early basic models for dry fabrics paved the way to their mechanical analysis. It started with the early attempt introduced in classical Peirce [32] model for monofilament plain weave fabrics defined at the mesoscale, later extended by Kemp [33], whose model was in turn criticized and modified by Hearle and Shanahan [34, 35].

For the developed CFRP composite materials, where different textile reinforcements are used for providing strength and stiffness, the mechanical behaviour of dry reinforcements 
(without resin) and that of composites made of reinforcements and matrix are quite different, since the matrix prevents motion between the fibres. Ishikawa and Chou [36] developed three 1D analytical geometrical models among the first that were designed especially for textile composites. These models study the knee phenomenon and in-plane stiffness coefficients of composites by using the classical laminate theory (CLT) introduced by Tsai and Hahn [37]. These models are categorized as 1D for they consider geometrical parameters in the loading direction only, which is a major limitation to accuracy. Naik et al. introduced a series of $2 \mathrm{D}$ analytical models for analysing the elastic moduli of plain weave lamina [38] and plain weave laminated composites [39], and also for optimizing laminate design [40].

In a further of development of the aforementioned 1D models [36], Raju and Wang [41] introduced a modified classical laminate theory for the 3D mosaic model. 3D geometrical descriptions of the repeating unit cell of plain weave, $5 \mathrm{H}$ and $8 \mathrm{H}$ satin weaves were developed assuming yarns with a rectangular cross-section and undulating in the gap between orthogonal tows. A number of 3D codes based on mathematical description of unit-cell were later introduced. Whitcomb et al. [42] developed the SAWC code, assuming that yarns follow sinusoidal paths. The finite element method was then used for predicting a stiffness matrix, but this code is limited in application to plain weave reinforcements.

Naik [43] developed the TEXCAD code, the most generalized model that addresses different reinforcements: plain weaves, $5 \mathrm{H}$ and $8 \mathrm{H}$ satin weaves, different forms of braids, laminates and interlock weaves. With a few geometrical parameters, yarn paths are defined as a combination of straight and sinusoidal segments. The yarn structure is modelled discretely. Applying the isostrain assumption throughout the unit cell, stiffness of the 
reinforcements of the composites are obtained along with the 3D stiffness constants, thermal expansion coefficients, initial failure and progressive failure strength.

Sheng and Hoa [44] developed a 3D micro-mechanical model to predict 3D stiffness of composites by applying variational potential energy and variational complementary energy methods. Implementation was based on 3D geometrical analysis of woven composites where, instead of using simplified sinusoidal functions to describe the yarn paths, the model identifies the paths by defining planar orientation angles and crimp function. In an simpler approach and basically applicable on dry fabrics used as reinforcements for composites, Hivet and Boisse [45] introduced the consistent 3D geometrical model for 2D dry nondeformed fabrics, calling it consistent as it guarantees no penetration between yarns.

Several 3D analytical models based on decomposition of the unit-cell were introduced. Hewitt et al. [46] developed computer-generated models of general fabric unit cells for 2D woven reinforcements. In the Porous Matrix Model, Kuhn and Charalambides [47] treated the composite unit-cell as 4 separate unidirectional layers of non-uniform thickness. The model aimed at producing piecewise continuous shape functions representing the top and bottom surfaces of each of the 4 layers by employing unit-cell dimensional parameters in describing yarn profiles.

Rao et al. [48] extended Kuhn and Charalambides' work [47] by modelling unit-cells of $4 \mathrm{H}, 5 \mathrm{H}$ and $8 \mathrm{H}$ satin weave composites while introducing a new middle matrix layer. The same modeling procedure was followed, first by describing the geometry of the unit-cell through a different approach where it was subdivided into binary sub-cells using the method introduced by Hewitt et al. [46]. 
On the other hand, software packages; mainly TexGen and WiseTex are available, provide a versatile tool for modelling any type of weave structure more efficiently with an easy implementation in the FEA methods. However challenges are envisaged with simulating deformed scenarios. TexGen was originally developed by Robitaille et al. [49] and later Sherburn [50] modified the code. The aim was to implement an algorithm for a generic geometric model at the level of the unit cell that can be applicable to a large array of 2D and 3D textile structures. Yarns are treated as solid volumes and the cross-section is approximated to be the smallest region that encompasses all of the fibres within the yarn. The yarn path is modelled by specifying its centerline, defined by a position in three dimensional space as a function of the distance along the yarn.

The WiseTex software package, introduced by Verpoest and Lomov [51], is assumed to be the only multi-scale numerical model integrating the micro-meso-macro characteristic hierarchy of textile reinforcements. Based on using the principle of minimum energy, and given the weave geometrical and mechanical parameters, the software defines all bend intervals and consequently computes the spatial displacement of fibres. For predicting the mechanical properties, some analytical models were integrated into the WiseTex software package, allowing virtual design of textile patterns. Among the integrated models are the TEXCOMP model [52-54], lattice Boltzmann solver [55] and LamTex model [56] predicting the stiffness of textile composites, permeability of textile reinforcements and internal geometry of textile laminates respectively.

Reviewed geometrical analytical models vary in their complexity and modelling strategies. They cover most of the commonly used weave reinforcements. However, no model can be generalized to include all 2D, 2.5D or 3D weave structures in any deformed state. 


\subsection{Preparation of Testing Coupons}

Hallal et al. [57] evaluated the available analytical models from a specific perspective by classifying them in terms of micro-macro homogenization methods, i.e. by applying CLT, iso-stress and/or iso-strain assumptions or inclusion method. The authors concluded that the CLT can be reliable in predicting stiffness properties in case of laminates or simple 2D geometrical structures where subdivision of the RVE is straightforward, whereas the isostrain assumption, while can be more generic, but lacks accuracy.

To simplify the calculations and assumptions, 2D laminated CFRP was selected to be the focus of this study. Stacking configuration replicated that appearing in Figure 2.3, however excluding the stitch featuring the $2.5 \mathrm{D}$ composite structures. The $2 \mathrm{D}$ laminated configuration having a cross-ply stacking sequence of $\left[0^{\circ} / 90^{\circ} / 0^{\circ} / 90^{\circ}\right] \mathrm{s}$ had been selected.

The material described in this chapter was used for quantitative analysis discussed in Chapter 4 of this thesis. The structure was obtained by stacking several laminae in the specified sequence to form the laminate. Each lamina, seen in Figure 2.5, features a large number of fibres pre-impregnated with a thin layer of resin matrix. It was cut into coupons using a waterjet machine. Dimensions of coupons appear in Appendix A.

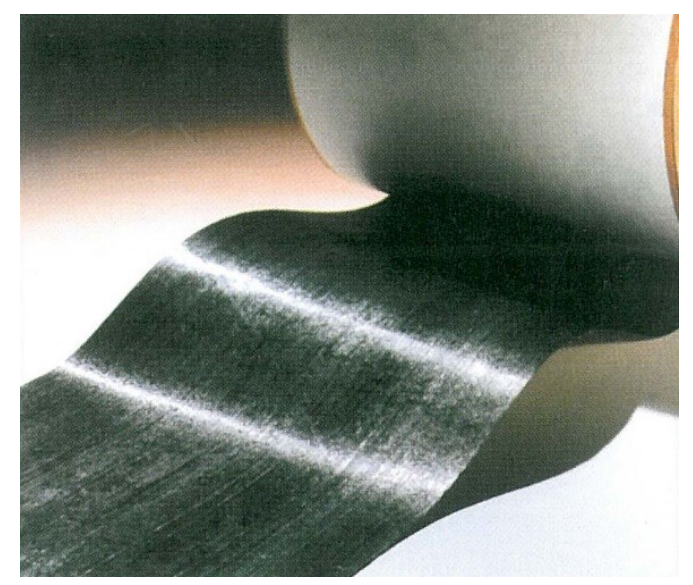

Figure 2.5: Prepreg carbon/epoxy [58] 


\subsubsection{Material Specifications}

Material used has a brand name: DA 409U/G35 150 Unidirectional Carbon/Epoxy Prepreg supplied by APCM [58], which is characterized by being a tough, versatile modified epoxy resin prepreg. Material was supplied in the form of a standard modulus carbon prepreg tape of specific area of $150 \mathrm{~g} / \mathrm{m}^{2}\left(0.060 \mathrm{lbs} / \mathrm{ft}^{2}\right)$. Resin content was $48 \%$ by weight and fibre volume fraction was 0.57 , whereas nominal laminate density was $1.96 \mathrm{~g} / \mathrm{cm}^{3}$, tow count was $12 \mathrm{~K}$ and fibre density was $1.77 \mathrm{~g} / \mathrm{cm}^{3}$ according to the supplier's specification sheet [58]. Other physical properties included a resin flow of $24 \%$, gel time of 12 minutes at $250^{\circ} \mathrm{F}$ and a shelf life of one year at $0^{\circ} \mathrm{F}$, six months at $40^{\circ} \mathrm{F}$ and thirty days at $75^{\circ} \mathrm{F}$.

Mechanical properties provided by the supplier included information on flexural strength of $224 \mathrm{ksi}(1544 \mathrm{MPa})$ and flexural modulus of $17.9 \times 10^{3} \mathrm{ksi}(123 \mathrm{GPa})$ both according to ASTM D 790. Beam Sheer was estimated being $13.8 \times 10^{3} \mathrm{ksi}$ (95 GPa) according to ASTM D 2344, while ASTM D 3039 lead to an estimation of tensile strength of $280 \mathrm{ksi}$ (1930 MPa) and tensile modulus of $18.8 \times 10^{3} \mathrm{ksi}(129 \mathrm{GPa})$. An average thickness of $0.375 \mathrm{~mm}$ was recorded upon investigating the samples in lab.

Material used, DA 409U/G35, is similar in mechanical properties to DA 4518U which was investigated by Rubio-González et al. [59]; both supplied by APCM [58]. Hence, published elastic properties were used: $\boldsymbol{E}_{1}=107.70 \mathrm{GPa}, \boldsymbol{E}_{2}=8.10 \mathrm{GPa}, \boldsymbol{v}_{12}=0.34, \boldsymbol{G}_{12}=3.85 \mathrm{GPa}$ and $\boldsymbol{\rho}=1505.8 \mathrm{~kg} / \mathrm{m}^{3}$. Where $\boldsymbol{E}_{1}, \boldsymbol{E}_{2}, \boldsymbol{v}_{12}, \boldsymbol{G}_{12}$ and $\boldsymbol{\rho}$ are Young's moduli in 2 orthogonal directions, Poisson's ratio, shear modulus and material density respectively [59].

Material specifications and its mechanical properties are listed in Appendix B of this work. 


\subsubsection{Dimensions of Testing Coupons}

The laminated panel had been manufactured as per ASTM standards $[60,61]$ at the NRC Composites facility in Ottawa. The material was laminated in typical cross-ply stacking sequence of $\left[0^{\circ} / 90^{\circ} / 0^{\circ} / 90^{\circ}\right] \mathrm{s}$. Each of the 8 plies was $0.375 \mathrm{~mm}$ thick, so the laminate plate had a thickness of $3 \mathrm{~mm}$ and a width of $200 \mathrm{~mm}$.

The plate was precisely cut by waterjet machine available in the Scientific Research Centre at Carleton University. It was cut into 8 coupons, Figure 2.6, and notched at mid-point of each side using a milling machine so as to study the effect of stress concentration factors and provide a predictable location for nucleation of fatigue damage. Adhering to ASTM standards $[60,61]$, the ratio of diameter/height of coupons was kept at 1.3 while the ratio of width/diameter was 6.2. Hence, coupons were of $177.8 \mathrm{~mm}$ length, $24 \pm 1 \mathrm{~mm}$ width and $3 \mathrm{~mm}$ thickness. Notch radius was $2 \pm 0.03 \mathrm{~mm}$ and area of cross section was $72 \mathrm{~mm}^{2}$.

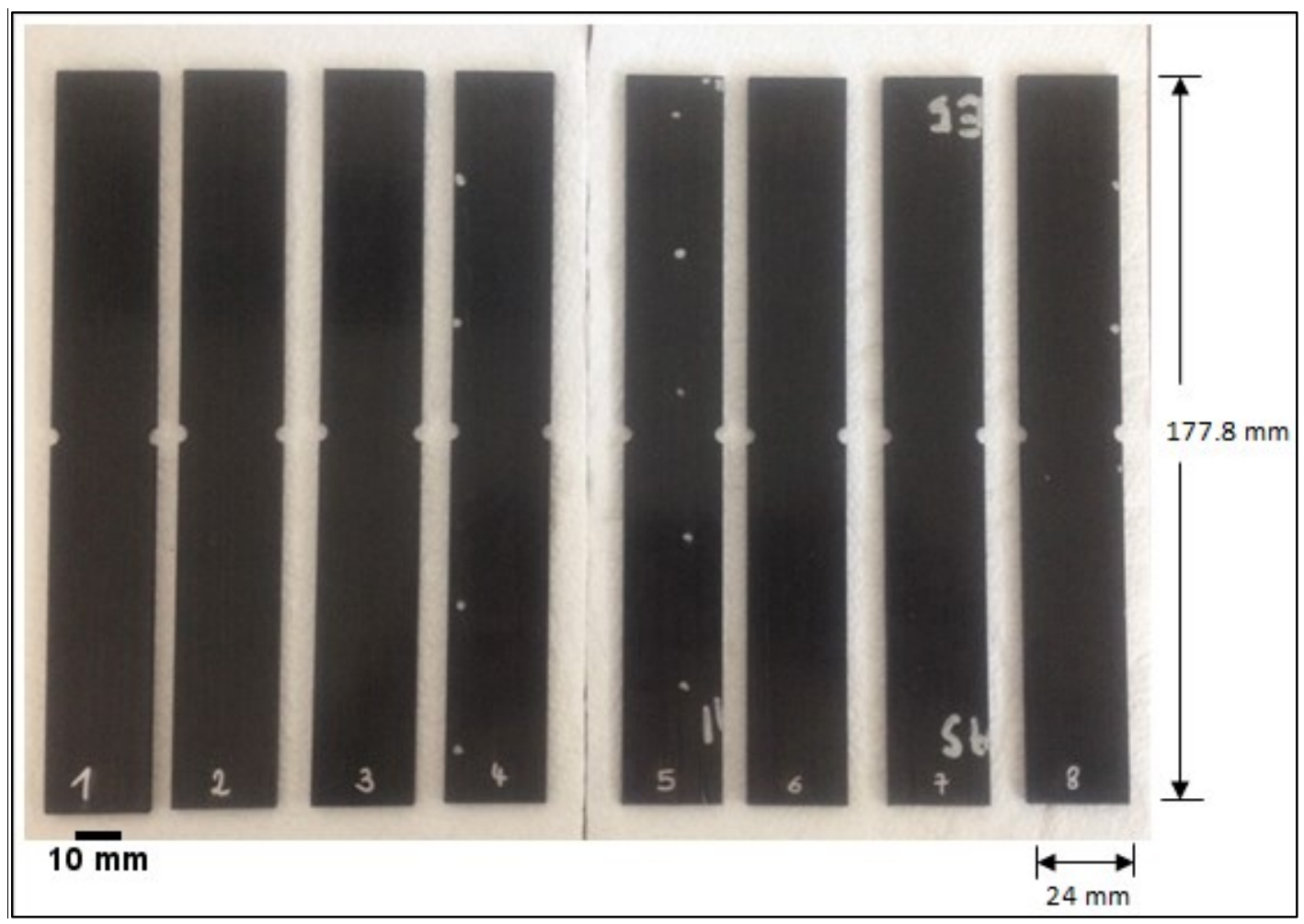

Figure 2.6: Eight fabricated coupons 
Dimensions appear in Figure 2.7, and a summary of the coupon specifications can be referred to in Appendix A. Using a Vernier caliper (accuracy of $\pm 0.03 \mathrm{~mm}$ ), the coupons were measured in order to verify their cut dimensions. The length was measured at 2 points, the width at 4 points and the notch radius at 1 point and the thickness at 5 points. The results are summarized Table 2.1. Coupons were of the same size, and coupon of the best quality was selected for fatigue testing and scanning.

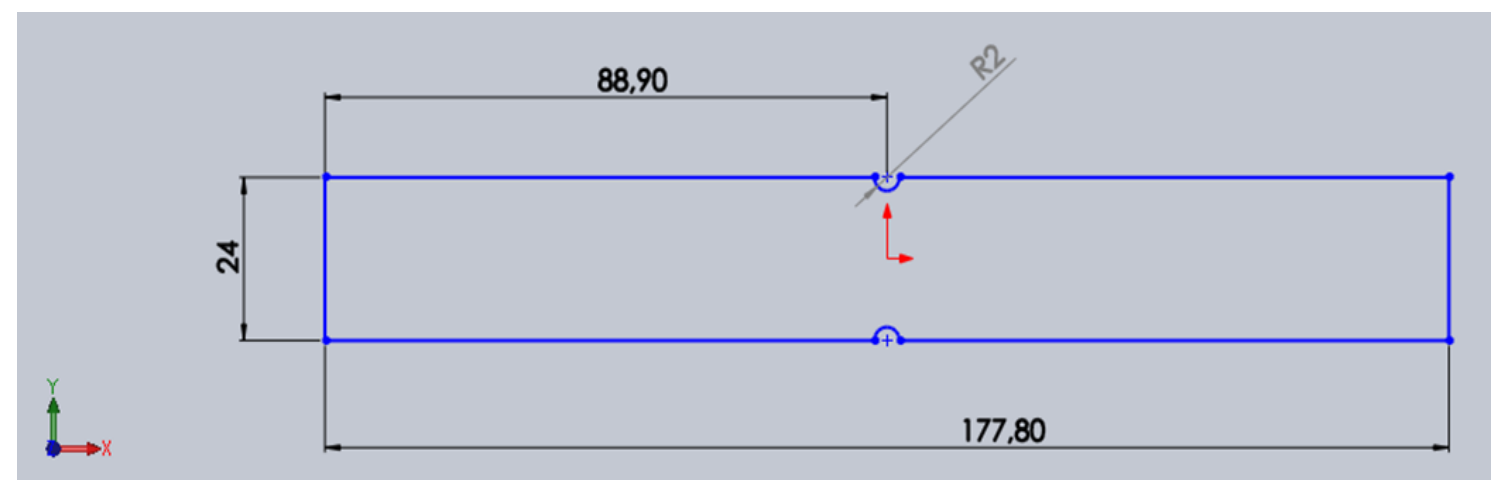

Figure 2.7: Dimensions of testing coupons (mm)

Table 2.1: Results of dimensions verification

\begin{tabular}{|c|c|c|c|c|c|c|c|c|c|c|}
\hline & Standard & 1 & 2 & 3 & 4 & 5 & 6 & 7 & 8 & Average \\
\hline Length $(\mathrm{mm})$ & 177.8 & 176.9 & 177.0 & 176.9 & 177.0 & 177.0 & 177.0 & 177.2 & 177.0 & 177.0 \\
\hline Width $(\mathrm{mm})$ & 24 & 24.14 & 24.13 & 24.13 & 24.12 & 24.13 & 24.11 & 24.14 & 24.10 & 24.12 \\
\hline $\begin{array}{c}\text { Notch radius } \\
(\mathrm{mm})\end{array}$ & 2 & 2.00 & 1.97 & 2.00 & 1.97 & 1.99 & 2.00 & 1.99 & 1.98 & 1.99 \\
\hline $\begin{array}{c}\text { Thickness } \\
(\mathrm{mm})\end{array}$ & 3 & 2.89 & 2.85 & 2.91 & 3.01 & 2.90 & 2.96 & 2.83 & 2.92 & 2.91 \\
\hline
\end{tabular}

\subsubsection{Quality Inspection of Testing Coupons}

A travelling microscope, Figure 2.8, was set up for rapid observation of external aspects of the coupons so as to verify quality standards. The microscope consists of a cast iron base, with a microscope tube attached to a 10x eyepiece and an $80 \mathrm{~mm}$ objective of high quality. The objective can be used in a vertical position as well as in horizontal observation during fatigue testing. A digital camera (Olympus SP 350, optical zoom 3x) was attached to the microscope and a light source was used to provide focused light. 


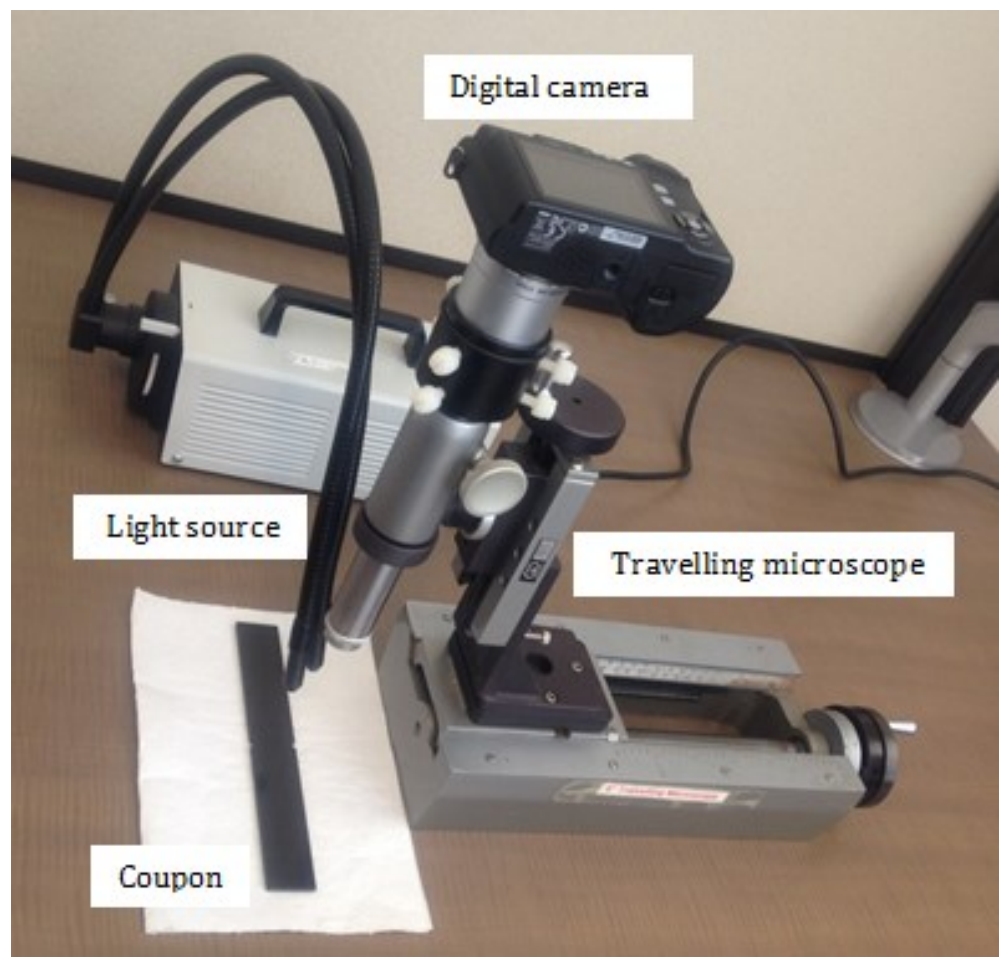

Figure 2.8: Travelling microscope set up to observe the external aspects of the coupons

Prelimenary observations confirmed the occurrence of delamination defects (separation of 2 adjacent plies, both along length and breadth of all coupons), Figure 2.9, induced by the cutting process. Frequent detection of delamination defects near the surface suggests the occurrence of more internal defects within the plies. Non-destructive visualization method was required to be adopetd in order to confirm the hypothesis, which was carried out by using the high resolution XR- microCT technique as discussed in Chapters 3 and 4 of this thesis.

Some cutting defects could be detected, Figure 2.10, especially in the notch areas. Mainly, most of the layers were not evenly laid in addition to a surface defect, interpreted as being fibre breakage, had been detected in coupon 4, Figure 2.11. Hence coupon number 4 was kept for static tensile testing to verify the maximum tensile strength, whereas coupon number 8 , visually having the best quality, was the one selected for further scanning. 


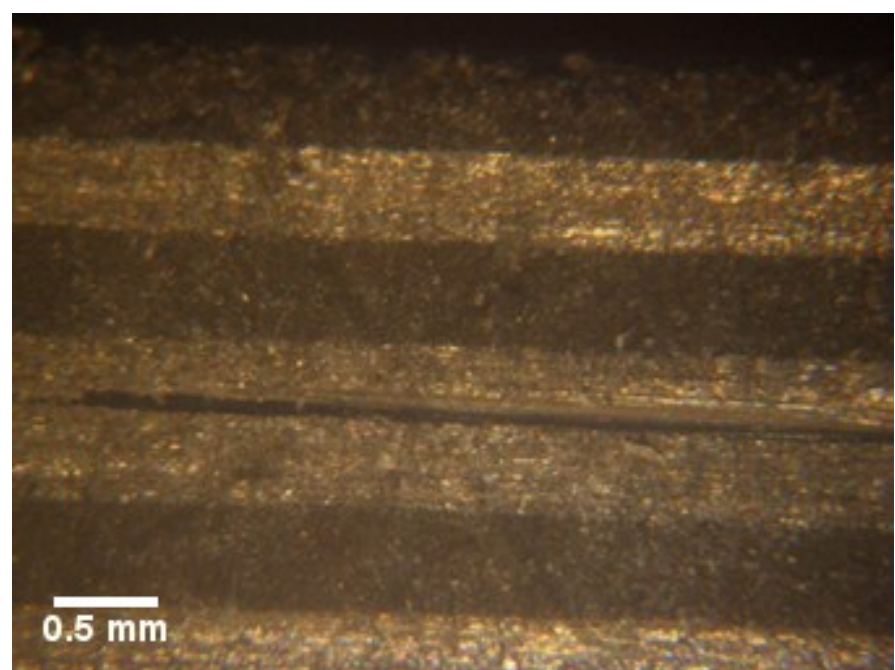

Figure 2.9: Delamination observed near the edge of coupon 6

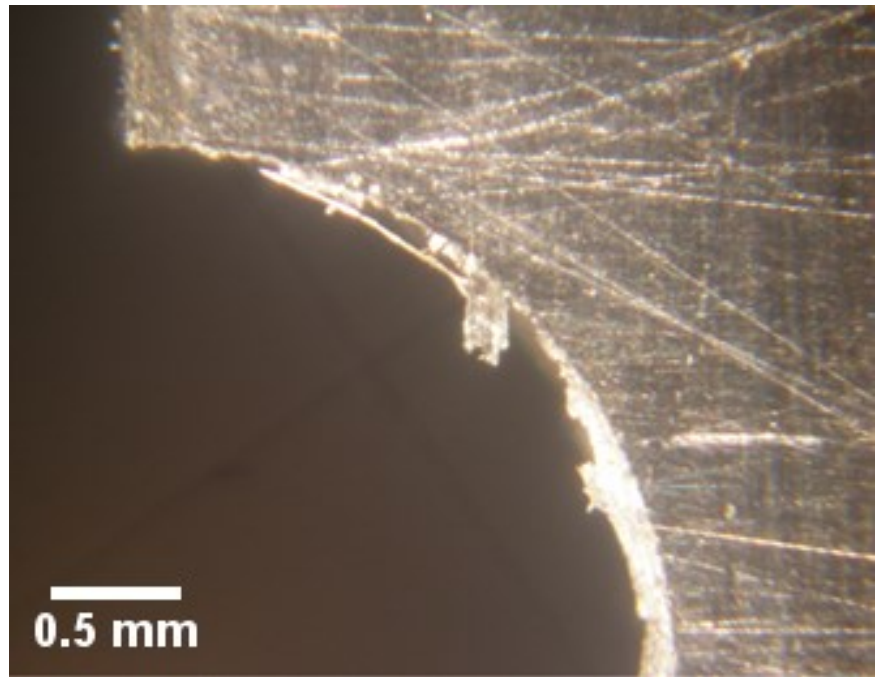

Figure 2.10: Cutting defects at the notch of coupon 7

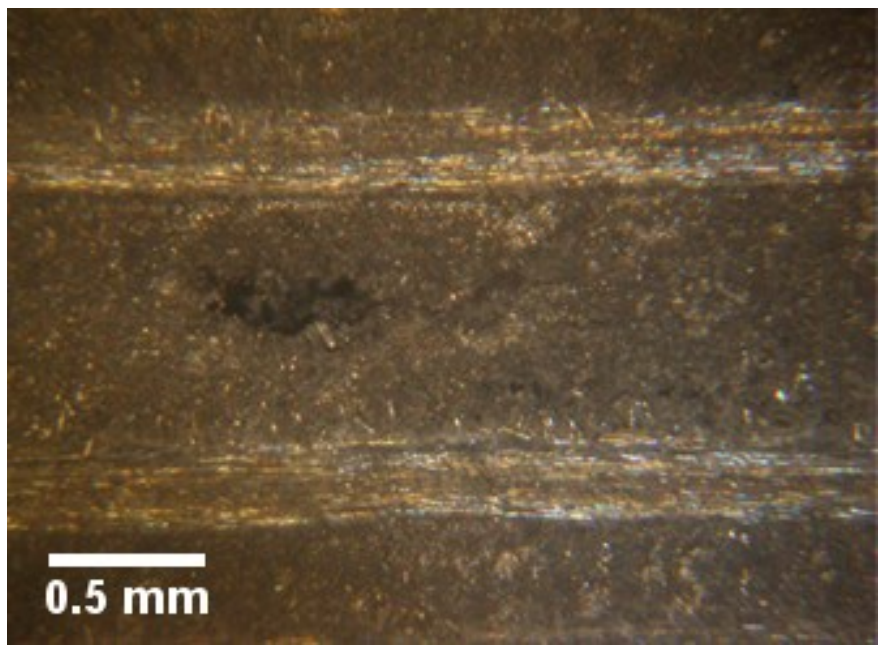

Figure 2.11: Observed surface defect in coupon 4 


\subsubsection{Material Stiffness Properties}

Calculations of ABD matrix were performed using the CLT method [37]. The ABD matrix is a $6 \times 6$ matrix that serves as a connection between the applied loads and the associated strains in the laminate. It essentially defines the elastic properties of the entire laminate. The composite material has the highest strength and modulus in the longitudinal direction of the fibres. Conversely, in the transverse direction, its strength and modulus are very low. Referring to section 2.3.1, published engineering constants [59] of each single ply in the composite lay-up were used throughout this study, following the general rules assuming that $E_{2}=E_{3}, v_{12}=v_{13}$ and $G_{12}=2 * G_{23}$.

For calculating the elastic properties of the entire 8-ply composite lay-up, the CLT [37] was used herein for processing calculations. Starting with a unidirectional single lamina, on-axis compliance terms, $S_{x x}, S_{y y}, S_{s s}, S_{y x}$ and $S_{x y}$, and on-axis stiffness terms, $Q_{x x}, Q_{y y}, Q_{s s}$, $Q_{y x}$ and $Q_{x y}$ were calculated by using the basic Equations 2.1 to 2.10 respectively. Hence, stresses or strains; whatever were unknown, could be calculated.

$$
\begin{array}{ccc}
S_{x x}=\frac{1}{E_{x}}, & \text { Equation 2.1 } & S_{y y}=\frac{1}{E_{y}}, \quad \text { Equation 2.2 } \\
S_{S S}=\frac{1}{E_{s}}, & \text { Equation 2.3 } & S_{y x}=-\frac{v_{x}}{E_{x}}, \text { Equation 2.4 } \\
S_{x y}=-\frac{v_{y}}{E_{y}}, & \text { Equation 2.5 } \\
Q_{x x}=m E_{x}, \text { Equation 2.6 } & Q_{y y}=m E_{y}, \text { Equation 2.7 } \\
Q_{s S}=E_{S}, \quad \text { Equation 2.8 } & Q_{y x}=m v_{x} E_{y}, \text { Equation 2.9 } \\
Q_{x y}=m v_{y} E_{X}, & \text { Equation 2.10 }
\end{array}
$$

Where $\quad m=\left(1-v_{x} v_{y}\right)^{-1}$ 
In addition, $U$ and $U$ " terms could be obtained using the so calculated terms, Equations 2.11- 2.20. The 4 unidirectional off-axis single laminae, stacked at $90^{\circ}, S_{i j}$ and $Q_{i j}$ terms were obtained, leading to calculating off-axis engineering constants of these 4 plies.

$$
\begin{array}{ll}
U_{1}=1 / 8\left(3 Q_{x x}+3 Q_{y y}+2 Q_{x y}+4 Q_{s s}\right), & \text { Equation 2.11 } \\
U_{2}=1 / 2\left(Q_{x x}-Q_{y y}\right), & \text { Equation 2.12 } \\
U_{4}=1 / 8\left(Q_{x x}+Q_{y y}+6 Q_{x y}-4 Q_{s s}\right), & \text { Equation 2.13 } \\
U_{3}=1 / 8\left(Q_{x x}+Q_{y y}-2 Q_{x y}-4 Q_{s s}\right), & \text { Equation 2.14 } \\
U_{5}=1 / 2\left(U_{1}-U_{4}\right) & \text { Equation 2.15 } \\
U^{\prime \prime}{ }_{1}=1 / 8\left(3 S_{x x}+3 S_{y y}+2 S_{x y}+S_{s s}\right) & \text { Equation 2.16 } \\
U^{\prime \prime}{ }_{2}=1 / 2\left(S_{x x}-S_{y y}\right) & \text { Equation 2.17 } \\
U^{\prime \prime}{ }_{3}=1 / 8\left(S_{x x}+S_{y y}-2 S_{x y}-S_{s s}\right) & \text { Equation 2.18 } \\
U^{\prime \prime}{ }_{4}=1 / 8\left(S_{x x}+S_{y y}+6 S_{x y}-S_{s s}\right) & \text { Equation 2.19 } \\
U^{\prime \prime}{ }_{5}=2\left(U^{\prime \prime}{ }_{1}-U^{\prime \prime}{ }_{4}\right) & \text { Equation 2.20 }
\end{array}
$$

For the symmetrical laminated plate, the terms $V_{1}{ }^{*}, V_{2}{ }^{*}, V_{3}{ }^{*}$ and $V_{4}{ }^{*}$ were calculated and used with $U$ terms and $U$ ” terms for calculating the normalized stiffness terms, $A_{i j} / h$ and normalized compliance terms, $a_{i j} * h$. Consequently, the $A$ matrix could be obtained by multiplying by the laminate thickness, $h$.

For obtaining the moment-curvature stiffness matrix, $D_{i j}$; the terms $W_{1}{ }^{*}, W_{2}{ }^{*}, W_{3}{ }^{*}$ and $W_{4}{ }^{*}$ were calculated and multiplied by moment of inertia, $I^{*}$, to obtain $W_{1}, W_{2}, W_{3}$ and $W_{4}$ terms. The latter were used with $U$ terms and $h^{*}$ to obtain the $D$ matrix. The moment-curvature compliance matrix, $d_{i j}$, could be hence obtained. The $B$ matrix which represents the coupling between axial and bending was null. 
Detailed calculations of laminate properties using the CLT are listed in Appendix C, whereas final results of $A$ and $D$ matrices can be seen in Figure 2.12, noting that units were in $\mathrm{GPa}$ and N.m respectively. Calculations were verified by applying an open-source software, eLaminate $\mathbb{C}$ [62]. Exactly the same results in $M P a$ and N.mm units were obtained, seen in Figure 2.13, which confirmed correct calculation procedures.

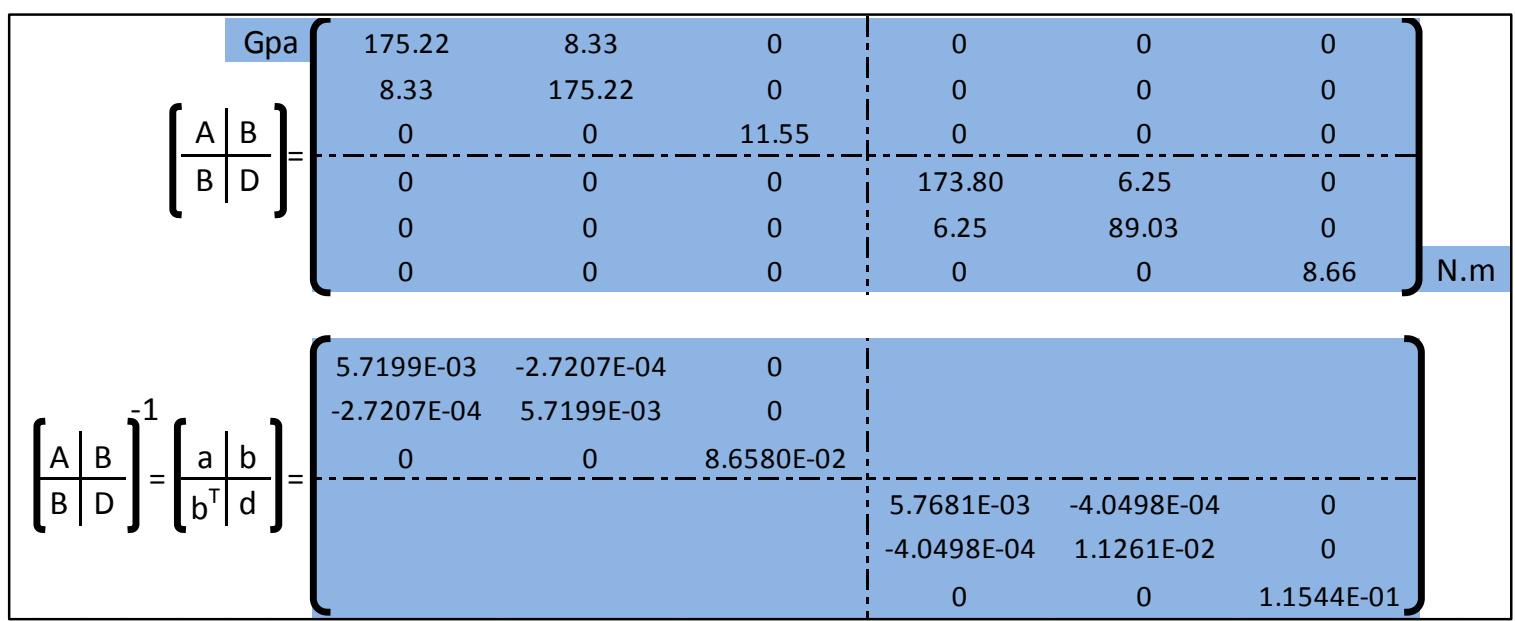

Figure 2.12: Engineering constants obtained from CLT calculations

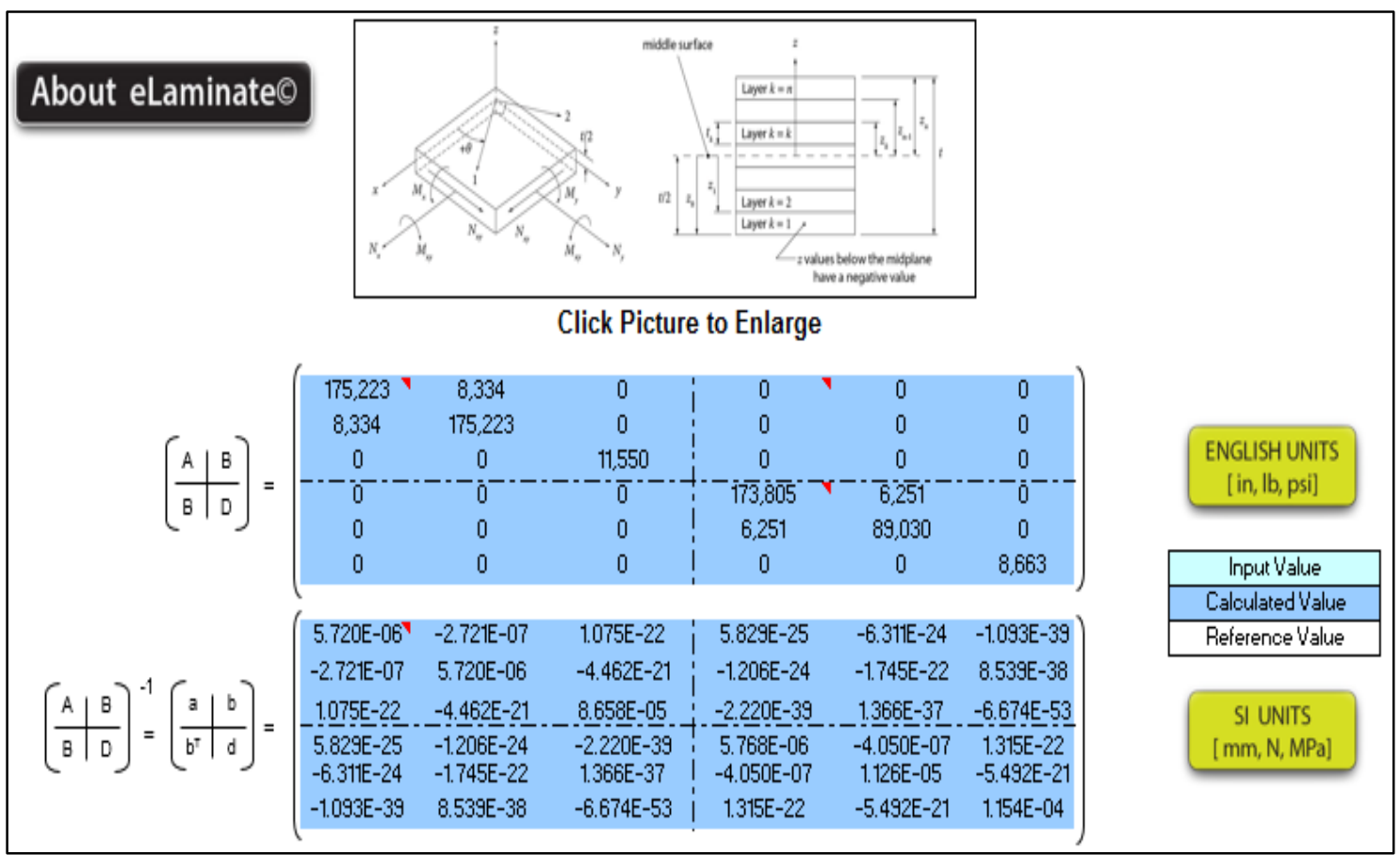

Figure 2.13: Engineering constants obtained from eLaminate (C) [62] 
Upon performing FEA simulations detailed in Chapter 6, single ply material constants were used as inputs in case of modelling composite lay-ups. Values of single ply material properties are listed in Appendix B. However, in case of assuming a homogeneous isotropic material in some of the simulations, average values were used to address the quasi-isotropic properties.

\subsection{Mechanical Testing}

\subsubsection{Identifying Static Strength}

A static tensile test had been carried out on coupon 4 for identifying the maximum static strength of the material. The test was performed on a hydraulic powered 810 Material Test System (MTS) having a maximum capacity of $100 \mathrm{kN}$. It involved applying a monotonic tensile load to the specimen in a load control mode until the point of fracture. Clamping pressure was $13 \mathrm{MPa}$, the traction speed was $0.3 \mathrm{~mm} / \mathrm{min}$. One data point was recorded per 20 second, where the displacement and the force were recorded. Strains were recorded by an extensometer of $0.5 \%$ precision, Figure 2.14, and also by using a Digital Image Correlation (DIC) method for verification.

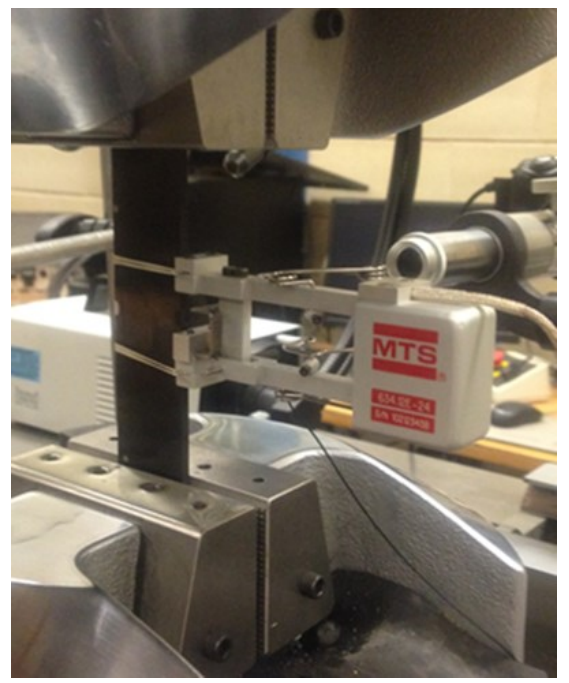

Figure 2.14: Extensometer mounted on a composite coupon during a static tensile test 
DIC set up included a digital camera (Olympus SP 350A) that was set on a tripod as well as a ring light (Digital DR-6000, LED Macro Ring Light, Vivitar) used to light the coupon. A MATLAB code was used to calculate vertical displacements and strains. In addition, a travelling microscope was mounted on the machine, keeping the base gripped and the microscope tube held in a horizontal position. The test set-up can be seen in Figure 2.15. It must be noted that either of the 2 light sources was switched off during the test so as not to heat the sample. The extensometer was removed after 10 recordings.

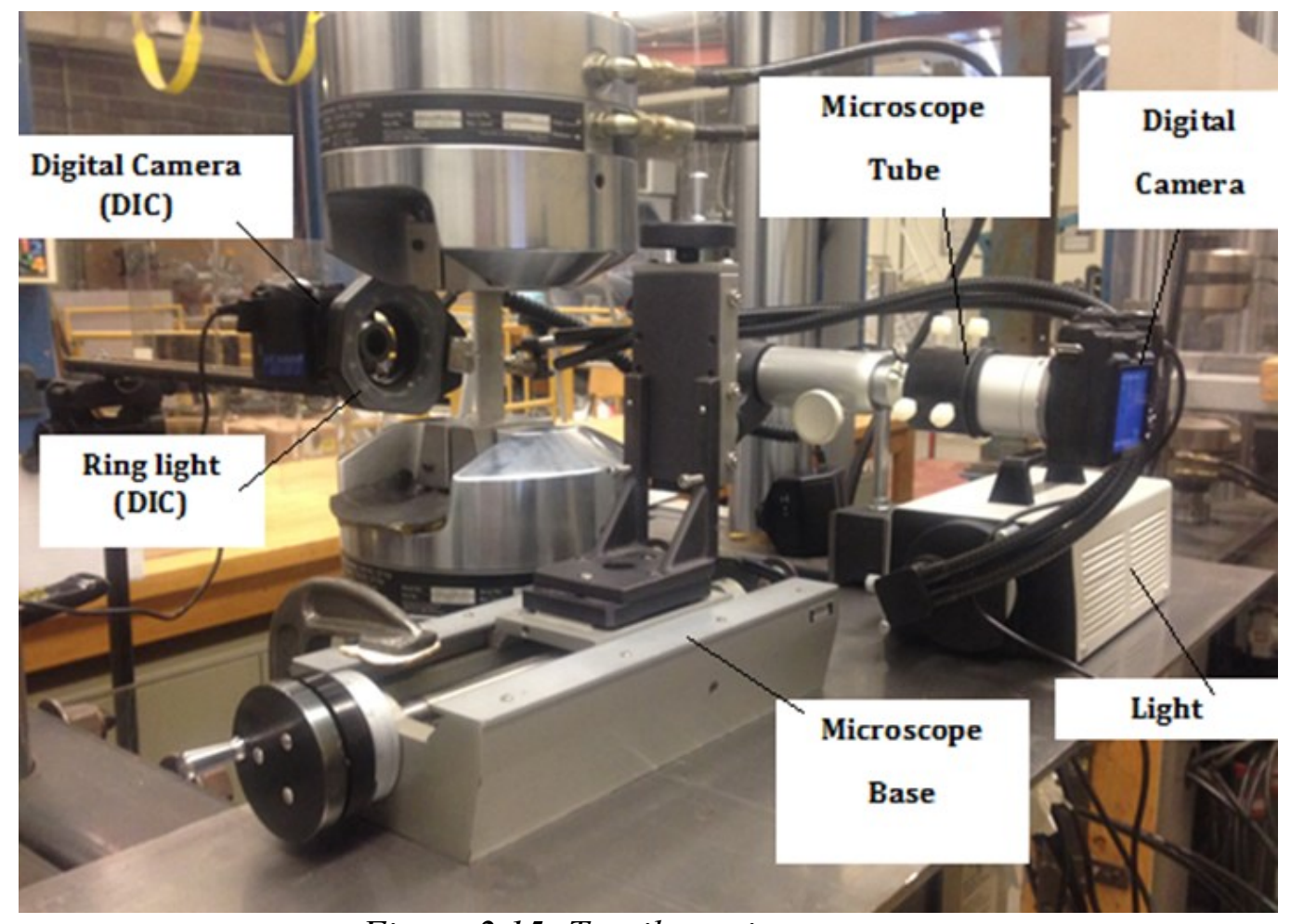

Figure 2.15: Tensile static test set up

An even failure at the mid-point of the notch can be seen in Figure 2.16. Failure occurred at a load of $35.585 \mathrm{kN}$, corresponding to a nominal stress of $494 \mathrm{MPa}$ considering a cross section area of $72 \mathrm{~mm}^{2}$. Recordings of the tensile static testing and extensometer are listed in Table 2.2, and a graph representing the stress- strain relationship appears in Figure 2.17. From the graph plot, maximum tensile strength of the laminate was $450 \mathrm{MPa}$. 


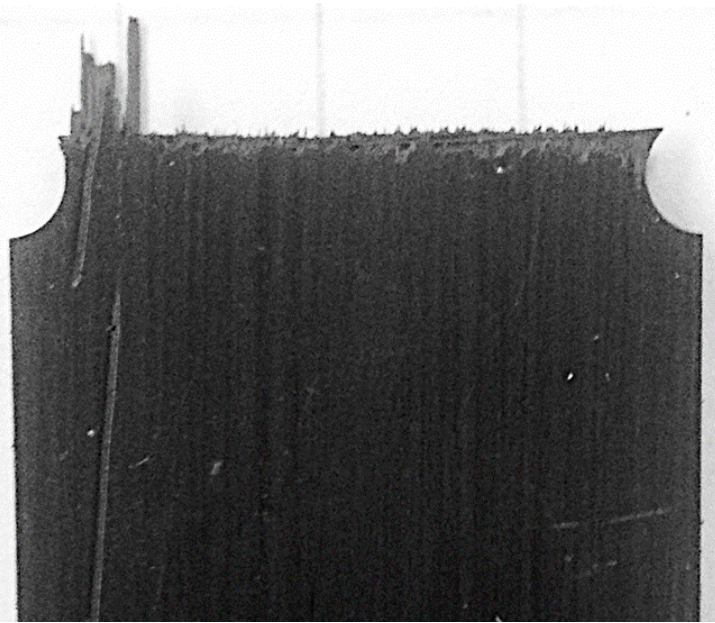

Figure 2.16: Coupon 4 after failure

Table 2.2: Recordings of tensile static test

\begin{tabular}{cccccc}
$\begin{array}{c}\text { Data } \\
\text { Point }\end{array}$ & Force $\left(\mathrm{lb}_{\mathrm{f}}\right)$ & Force $\mathrm{k}(\mathrm{N})$ & $\begin{array}{c}\text { Nominal Stress } \\
(\mathrm{MPa})\end{array}$ & $\begin{array}{c}\text { Displacement } \\
(\mathrm{mm})\end{array}$ & $\begin{array}{c}\text { Strain } \\
(\%)\end{array}$ \\
1 & 1500 & 6.672 & 92.671 & 0.239 & 0.194 \\
2 & 2000 & 8.896 & 123.561 & 0.329 & 0.263 \\
3 & 2500 & 11.120 & 154.452 & 0.425 & 0.334 \\
4 & 3000 & 13.344 & 185.342 & 0.524 & 0.407 \\
5 & 3500 & 15.568 & 216.232 & 0.624 & 0.488 \\
6 & 4000 & 17.792 & 247.123 & 0.73 & 0.568 \\
7 & 4500 & 20.016 & 278.013 & 0.838 & 0.649 \\
8 & 5000 & 22.241 & 308.904 & 0.962 & 0.739 \\
9 & 5500 & 24.465 & 339.794 & 1.088 & 0.834 \\
10 & 6000 & 26.689 & 370.685 & 1.216 & 0.923 \\
11 & 6500 & 28.913 & 401.575 & 1.365 & \\
12 & 7000 & 31.137 & 432.465 & 1.536 & \\
13 & 7500 & 33.361 & 463.356 & 4.047 & \\
14 & 8000 & 35.585 & 494.246 & 4.171 & \\
\hline 12 & & & & & \\
\hline
\end{tabular}




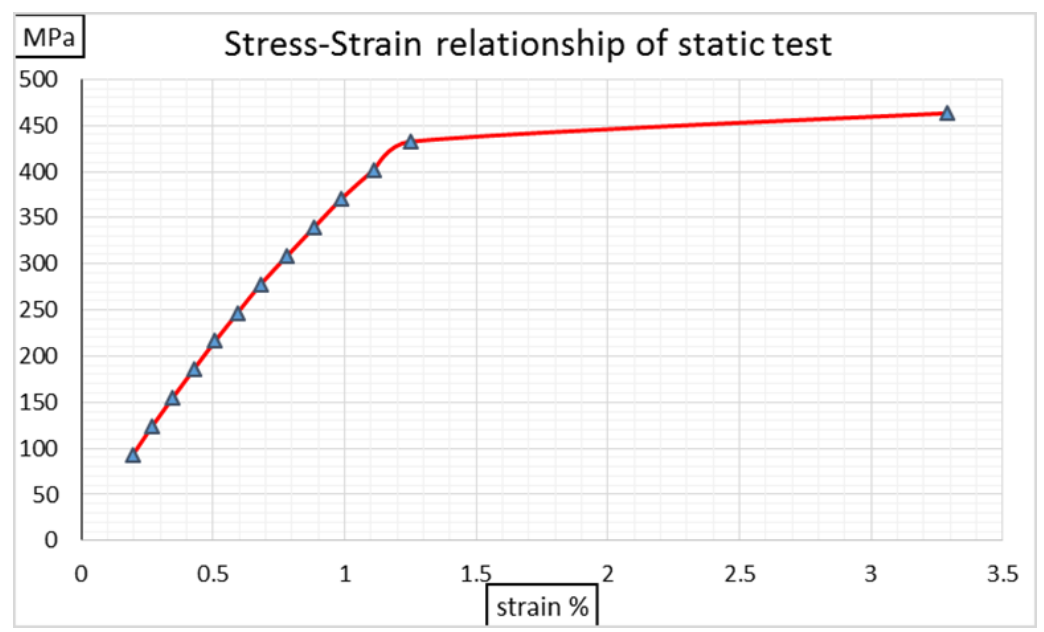

Figure 2.17: Stress-strain data recorded during the tensile static test

\subsubsection{Fatigue Spectrum}

Each structural part in the aircraft is subjected to a significant load variation over time, referred to as spectrum. Therefore, in order to accurately study the fatigue behavior of a specific part in service, it is crucial to obtain the load spectrum for that part. Mainly 2 spectra; TWIST (Transport Wing Standard Load) and FALSTAFF (Fighter Aircraft Loading Standard for Fatigue) can be used to represent the lower wing root panel [63].

For simplification, average values of peaks and valleys were determined from the original spectra, and used to set the maximum and minimum stresses for creating normalized spectra [64]. Typical cyclic loading parameters are shown in Figure 2.18, whereas properties of the normalized main spectra are summarized in Tables 2.3 and 2.4.

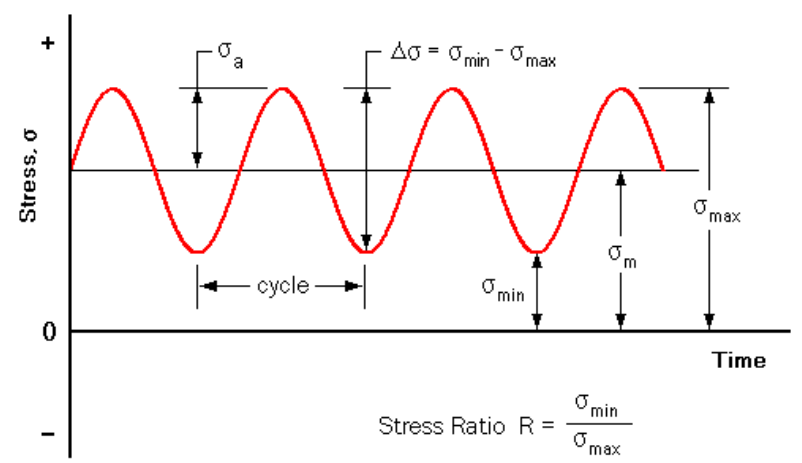

Figure 2.18: Typical cyclic loading parameters 
Table 2.3: Properties of normalized main spectra [64]

\begin{tabular}{|c|c|c|}
\cline { 2 - 3 } \multicolumn{1}{c|}{} & TWIS T Spectrum & FALS TAFF S pectrum \\
\hline $\begin{array}{c}\text { Absolute Maximum } \\
\text { Stress } \sigma_{\max }\end{array}$ & 2.6 & 6.158 \\
\hline $\begin{array}{c}\text { Absolute Minimum } \\
\text { Stress } \sigma_{\min }\end{array}$ & -0.6 & -1.644 \\
\hline $\begin{array}{c}\text { Mean Stress } \\
\sigma_{\mathrm{m}}\end{array}$ & 0.993 & 1.379 \\
\hline $\begin{array}{c}\text { Alternating Stress } \\
\sigma_{\mathrm{a}}\end{array}$ & 0.245 & 0.618 \\
\hline $\begin{array}{c}\text { Stress Ratio } \\
\text { R }\end{array}$ & 0.604 & 0.381 \\
\hline
\end{tabular}

Table 2.4: Properties of normalized main spectra per flight phase [64]

\begin{tabular}{|c|c|c|c|c|c|c|}
\hline \multirow{2}{*}{ Flight Condition } & \multicolumn{3}{|c|}{ TWIST Spectrum } & \multicolumn{3}{c|}{ FALSTAFF Spectrum } \\
\cline { 2 - 7 } & $\sigma_{m}$ & $\sigma_{a}$ & $R$ & $\sigma_{m}$ & $\sigma_{a}$ & $R$ \\
\hline Cruise Flight & $\mathbf{1 . 0 0 0 0}$ & $\mathbf{0 . 2 2 2 0}$ & $\mathbf{0 . 6 3 6 7}$ & $\mathbf{1 . 0 0 0 0}$ & $\mathbf{0 . 3 7 7 5}$ & $\mathbf{0 . 4 5 1 9}$ \\
\hline Cground Roll & $\mathbf{0 . 2 5 0 0}$ & $\mathbf{0 . 7 5 0 0}$ & $-\mathbf{0 . 5 0 0 0}$ & $\mathbf{- 0 . 0 3 8 0}$ & $\mathbf{0 . 4 7 1 8}$ & $\mathbf{- 1 . 1 7 5 2}$ \\
\hline Mane uvering & ---- & --- & ---- & 1.7549 & 1.5098 & $\mathbf{0 . 0 7 5 1}$ \\
\hline Light Gust & $\mathbf{0 . 9 9 7 3}$ & $\mathbf{0 . 2 6 8 5}$ & $\mathbf{0 . 5 7 5 8}$ & $\mathbf{1 . 1 9 1 5}$ & $\mathbf{0 . 4 7 2 9}$ & $\mathbf{0 . 4 3 1 8}$ \\
\hline Stormy Weather & $\mathbf{1 . 0 2 5 6}$ & $\mathbf{0 . 3 8 9 7}$ & $\mathbf{0 . 4 4 9 3}$ & 1.5776 & $\mathbf{0 . 7 5 6 2}$ & $\mathbf{0 . 3 5 2 0}$ \\
\hline
\end{tabular}

\subsubsection{Fatigue Testing}

Average cruise data were selected for constant amplitude fatigue testing where mean stress,

$\boldsymbol{\sigma}_{\boldsymbol{m}}$, was $30.00 \mathrm{ksi}(207 \mathrm{MPa})$, amplitude stress, $\boldsymbol{\sigma}_{\boldsymbol{a}}$, was $6.66 \mathrm{ksi}(46 \mathrm{MPa})$ and stress ratio, $\boldsymbol{R}$, was 0.63 . Hence maximum stress $\boldsymbol{\sigma}_{\boldsymbol{m a x}}$ was $36.60 \mathrm{ksi}(253 \mathrm{MPa})$ and $\boldsymbol{\sigma}_{\boldsymbol{m} \boldsymbol{i n}}$ was $23.10 \mathrm{ksi}$ (160 MPa). High cycle fatigue testing was performed.

By applying the basic relationship: $\boldsymbol{\sigma}_{\boldsymbol{i}}=\boldsymbol{P}_{\boldsymbol{V}} / \boldsymbol{A}$, the equivalent loads were $\boldsymbol{P}_{\boldsymbol{m a x}}=18.22 \mathrm{kN}$, $\boldsymbol{P}_{\boldsymbol{m i n}}=11.52 \mathrm{kN}\left(\right.$ tension - tension loading), $\boldsymbol{P}_{\boldsymbol{m}}=14.90 \mathrm{kN}$ and $\boldsymbol{P}_{\boldsymbol{a}}=3.31 \mathrm{kN}$.

Data of constant amplitude cyclic stress appear in Table 2.5, and applied loads on the fatigue testing frame are listed in Table 2.6. 
Fatigue uniaxial loading test was conducted in a load-control mode on a servo-hydraluic material test frame at Carleton University. Testing was performed at a loading frequency of $10 \mathrm{~Hz}$ with a sinusoidal waveform. Following ASTM standard [65] for evaluating data acquisition systems used in cyclic fatigue and fracture mechanics testing, the minimum bandwidth was $100 \mathrm{~Hz}$, the minimum data rate was 500 points/s and the standard deviation of noise level did not exceed $0.2 \%$.

Table 2.5: Data of cyclic loading Cyclic Stress

\begin{tabular}{|c|cccc|}
\hline \multirow{3}{*}{ Stress Level - R } & Max stress & Min stress & Mean Stress & Amplitude \\
& $\mathrm{MPa}$ & $\mathrm{MPa}$ & $\mathrm{MPa}$ & $\mathrm{MPa}$ \\
& $\sigma(\max )$ & $\sigma(\min )$ & $\sigma(\mathrm{m})$ & $\sigma(\mathrm{a})$ \\
\hline 0.63 & 253.00 & 160.00 & 207.00 & 46.00 \\
\hline
\end{tabular}

Table 2.6: Actual applied loads on fatigue testing frame

\begin{tabular}{|c|cccc|}
\cline { 2 - 5 } \multicolumn{1}{c|}{} & \multicolumn{4}{c|}{ Load Frame } \\
\hline \multirow{3}{*}{ Stress Level- $\mathrm{R}$} & Max Load & Min Load & Mean load & Amplitude \\
& $\mathrm{kN}$ & $\mathrm{kN}$ & $\mathrm{kN}$ & $\mathrm{kN}$ \\
& $\mathrm{P}(\max )$ & $\mathrm{P}(\mathrm{min})$ & $\mathrm{P}(\mathrm{m})$ & $\mathrm{P}(\mathrm{a})$ \\
\hline 0.63 & 18.22 & 11.52 & 14.90 & 3.31 \\
\hline
\end{tabular}

Axial strain was monitored using an extensometer recording an average of $0.018 \mathrm{~mm} / \mathrm{mm}$. Tests were performed at ambient temperature. Fatigue testing was interrupted at 200,000, $400,000,600,000,800,000,1,200,000$ and 1,800,000 cycles. At each fatigue phase, XRmicroCT scanning was performed on ultra-high resolution SkyScan 1173; the sample was studied at 7 phases of its fatigue life starting with the pristine state. 


\subsection{High Resolution X-ray Micro Computed Tomography}

Scanning was performed on an ultra-high resolution SkyScan 1173 XR-micro Computed Tomography (XR-microCT) unit available in the Department of Earth Sciences at Carleton University, Figure 2.19. Analysis of scans, detailed over the next 3 chapters of this work, provided information on propagation of initially occurring cracks throughout fatigue life at the specified intervals.

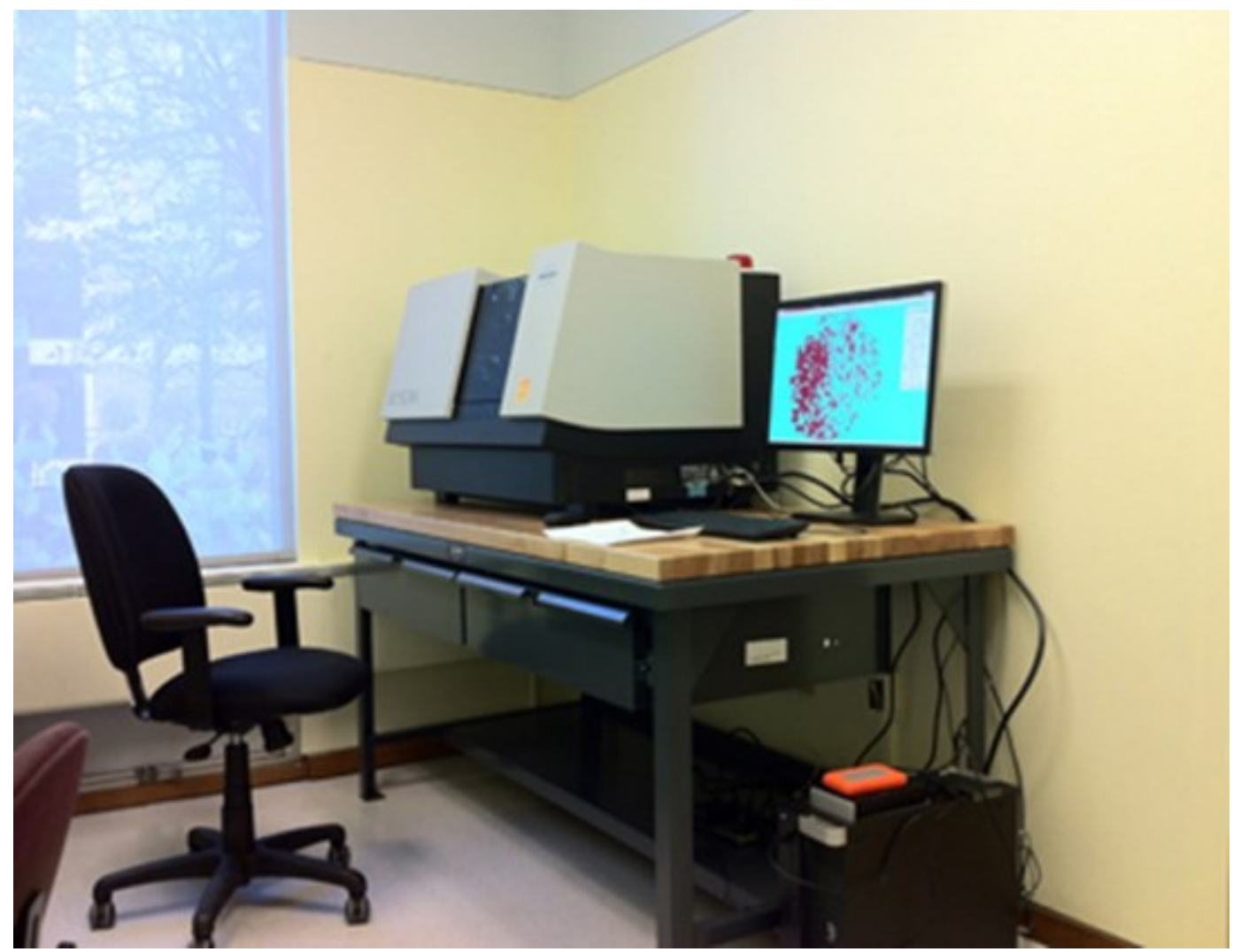

Figure 2.19: Ultra- high resolution X-ray microCT, Carleton University

The scanning unit featured a camera pixel size of $50.0 \mu \mathrm{m}$, a source voltage of $70 \mathrm{kV}$, and a source of $114 \mu \mathrm{A}$. Each sample was rotated in the X-ray beam over $360^{\circ}$ in $0.2^{\circ} /$ step using $1 \mathrm{~mm}$ - thick aluminum filter. The thickness of each slice was $12.08 \mu \mathrm{m}$ defining a spatial resolution of the 3D image of $12.08 \mu \mathrm{m}$ per voxel, hence image pixel size was 12.08 
$\mu \mathrm{m}$. Scanning time was approximately 2.6 hours for each sample. Post-processing of images was performed using Skyscan (NRecon, CTan, CTvox and DataViewer) software [66]. CTAnalyzer software included in the microCT package is a fast tool for providing STL surface meshed files that can be exported to Abaqus software, as well as providing other useful information like porosity percentage. CTVox also included in the microCT package provide a transparency-controlled volume rendered 3D images allowing one to visualize the cracks and qualitatively assess void content in $3 \mathrm{D}$ and trace their propagation. A raw scan appears in Figure 2.20.

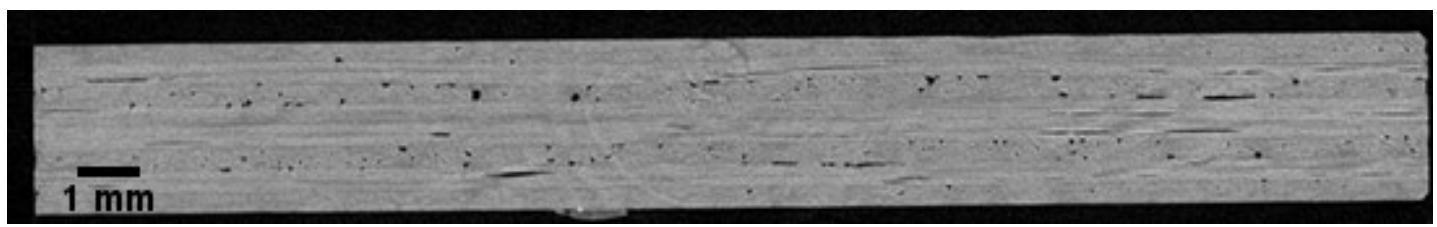

Figure 2.20: Ultra- high resolution $X$-ray raw image 


\section{Chapter 3:}

\section{Investigation of Fatigue Behaviour}

\subsection{Introduction}

Optical microscopy is often used to analyze the microstructure of materials and may be considered a destructive testing (DT) method. As the term implies, the method involves cutting the sample, polishing or preparing it for testing. The sample preparation procedure may also damage the internal architecture of interest. However, optical microscopy enables a detailed 2D analysis of microscopic features allowing for thorough study and eventual prediction of mechanical behaviour of materials. Alternatively, different non-destructive testing (NDT) methods used in assessing the microscopic 3D structure of materials are introduced in literature. These techniques are mainly categorized as based on analysis of visual, radiation, electromagnetic, mechanical vibration, optical and thermal signals [67].

Nevertheless, experimentally validated failure simulation models are not abundant in the literature for complex CFRP laminates. Nor is there abundant quantitative data capable of defining the microscopic characteristics for damage of such orthotropic laminates. Hence, 
early prediction and modelling of damage nucleation and subsequent evolution is precluded due to insufficient experimental data and verified models.

In this chapter, a qualitative visualization analysis was carried out for 3D microscopic damage behaviour observed in CFRPs subjected to constant amplitude fatigue loading. Probing the evolution of the cracks and damage was performed using Skyscan 1173 XRmicroCT testing unit previously described in Chapter 2. It was shown to be a reliable NDT tool for qualitatively visualizing and monitoring damage mechanisms relative to the fatigue life.

Further, a procedure to export 3D microCT scans to commercial FEA tool; i.e. Abaqus was carried out to assess the reliability of FEA modelling in addressing 3D fatigue problems. The proposed procedure for exporting data involved available tools which were discussed and assessed herein.

\subsection{Review of Literature}

In the context of CFRPs, manufacturing quality and in-service inspection are conducted routinely using ultrasonic techniques (UT), acousto-ultrasonics (AU); that is ultrasonic characterization combined with acoustic emission (AE) monitoring (a mechanical vibration technique), shearography (an optical technique) and infrared thermography (a thermal technique) [68-70].

These techniques have proven to be the most successful and widely used techniques and were implemented for visual probing of structures and quality control inspection of production parts. Complementary to the on-line inspection, these techniques are also implemented in the laboratory context for monitoring the mechanical behaviour of PMCs. 


\subsubsection{Implementation of Non-destructive Testing Tools}

Following a common procedure, several research teams analyzed temperature mappings and $\mathrm{AE}$ measurements and attributed their fluctuations to the number of loading cycles in fatigue testing [71], or to damage modes [72]. A relevant study was conducted by Loutas et al. [73] to acquire quantifying data relative to damage development stages in composites through analyzing $\mathrm{AU}$ and $\mathrm{AE}$ signals recorded during fatigue tests of quasi-isotropic CFRP. Typical 24 parameters of AU waveforms were calculated and plotted versus time showing 3 phases that can be correlated to the phases of fatigue life; initiating with matrix cracking, developed into delamination and finally significant fibre breakage to failure.

A quantification of the geometrical features associated with delamination defects was attempted by DeAngelis et al. [74] by implementing vibration excited digital shearography. The flexural strain profiles were derived from the recorded resonant frequencies corresponding to each defect. The depths and sizes of artificial holes that were to replicate these defects were assessed following an optimization algorithm through minimizing the difference between the experimental resonant frequencies applied for excitation and those obtained by theoretical computational methods.

A different approach was introduced by Cuadra et al. [75] who recently introduced a combination of 3 techniques; acoustic emission, digital image correlation and infrared thermography for quantifying defects in glass FRP composites subjected to tensile and fatigue loads. The obtained mappings of thermal and strain signals monitored the development of damage, and provided a correlation between strain behaviour of composites and the number of fatigue cycles. 
Rather than being combined with other NDT methods, infrared thermography can be also used independently for studying the behavior and defects in composites and is considered one of the NDT techniques that proved reliable in the recent years [70]. It provides thermal mapping of stimulated objects emitting or transmitting thermal energy relative to their emissivity coefficient. The thermally stimulated objects are visualized in either of 2 modes: the through-mode or transmission mode, where the object is placed between the heat excitation source and the infrared camera, and the face-mode or reflection mode having both the heat excitation source and the camera positioned on the same side of the object. In either visualization mode, the excitation waveform is applied following either of 2 main techniques; pulse (flash or transient) or lock-in thermography. The difference between these techniques lies in the duration of the excitation, varying from milliseconds to seconds in the case of the pulse flash technique to seconds to minutes in the case of the pulse transient technique, whereas for the lock-in technique the excitation waveform is sinusoidal or rectangular and its duration ranges from seconds to hours [76]. The typical duration for each technique is one of the major factors governing the choice of the technique to be applied in a particular case. For instance, the thermal propagation time needed for reaching a depth of $2 \mathrm{~mm}$ in composites materials is estimated to be about $30 \mathrm{~s}$, so the transient thermography is applied in this case. The thermal flow through a defective sample is partially obstructed by flaws causing localized increase in transient temperatures above the defects, showing hot spots in the thermal gradient [67].

\subsubsection{Implementation of X-ray MicroCT Technique}

X-ray micro-computed tomography (XR-microCT) is currently the only practical and readily available NDT method capable of creating digital cross-sections of a 3D-object that 
later can be used to directly recreate a virtual model of the internal structure. It is a radiation technique that is a derivative of the classic medical CT method [77], however at a higher resolution. X-rays are used to capture a large number of radiographic projections of the sample at different angular positions up to $360^{\circ}$. The concept is depicted in Figure 3.1; showing that as X-rays pass through a structure, they are absorbed where the level of absorption is dependent on the physical density of the material. The radiography method uses ionizing radiation through a structure where the level of absorption of the radiation is recorded by film or an electronic sensor on the opposite side of the structure.

The level of contrast on the recording film or sensor is dependent on the level of radiation that has passed through the structure. The presence of varying local physical densities in the composite structure will thus appear as a different contrast on the film. Thus, defects that have different physical densities to the resin and the fibres will be detected on film when oriented as the same plane as the transmitted beam. By using the isotropic resolution offered by microCT, the transverse sections are reconstructed, and then stacked to obtain a volumetric representation of the sample and to visualize the internal structure.

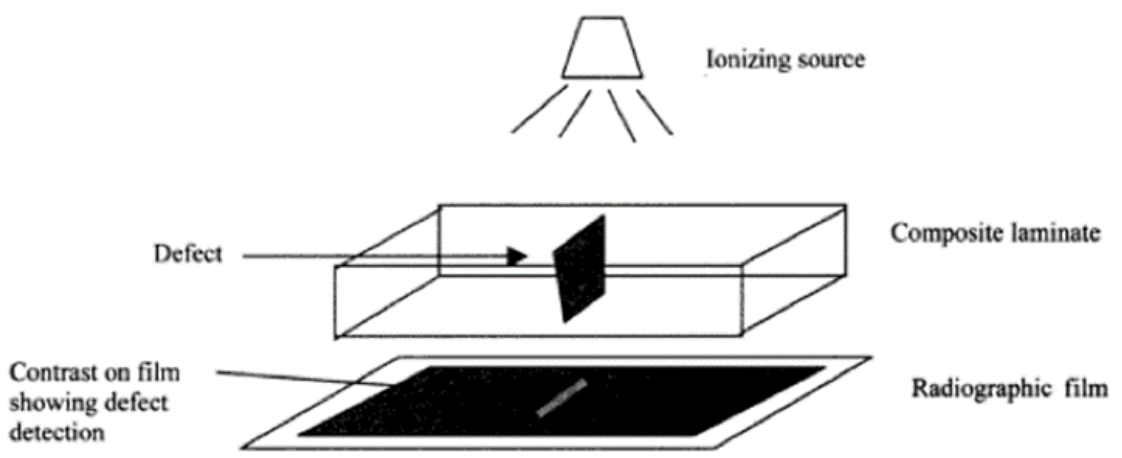

Figure 3.1: Schematic diagram of application of X-ray, modified from [78]

Buffiere et al. [78] presented an explanation of the microCT principle and its potential applications. This technique was employed by a number of research teams $[21,79,80]$ for 
providing a 3D analysis of the architectural geometry of dry textile preforms in pristine and deformed states serving as basis for permeability studies, and the same technique was used towards a quantitative estimation of fibre distribution and gaps caused by sewing tension in dry carbon preforms [81]. However, using this technique for defining geometrical structures may present difficulties in determining tow interfaces; a problem that may be overcome by tow coating [82].

A number of attempts for investigating damage in fibre reinforced polymer composites have been conducted using the microCT technique $[83,84]$. Little et al. [85] proved the reliability of microCT to characterize voids in CFRP, whereas Cosmi and Bernasconi [86] used it in demonstrating a qualitative assessment of the density of micro-voids relative to the number of fatigue cycles.

An advanced version of Synchrotron Radiation Computed Tomography (SRCT) provides what is considered to be the most well developed $3 \mathrm{D}$ experimental results that have been acquired to date. Wright et al. [87, 88] used the SRCT technique for qualitatively characterizing mechanisms of damage initiation in notched laminated CFRP composites subjected to tensile loading.

Scott et al. [89] provided an identification of damage modes relative to the percentage of final failure load by employing the same technique; they proved that transverse ply cracks occur at an earlier stage of loading than that causing splits, delaminations and then fibre breakage, respectively. Scott et al. [90] used the quantitative results obtained in [89] to validate an FEA multiscale model developed by Blassiau [91] for predicting failure in laminated composites in terms of the number of broken fibres. 


\subsection{Qualitative analysis of 3D rendered images}

The aforementioned techniques provide detection and monitoring of damage progression as a function of the mechanical parameters of the CFRP composites. Nevertheless, experimental visualization capable of defining the characteristics of initial microscopic damage evolution is not well documented in the literature except via destructive 2D optical and 3D scanning electron microscopy methods.

Hence, microCT testing in Carleton's newly acquired microCT facility is introduced in this work as a means of probing the evolution of cracks in the micro structure of CFRP composite subjected to constant amplitude fatigue loading. Images post-processing was performed using Skyscan software packages [66] and Avizo software [92]. Qualitative analysis detailed hereunder was introduced in SAMPE Conference [19].

For this analysis, 4 samples of 4 unidirectional plies $[0 / 90]_{s}$ were tested each at a different fatigue interval for preliminary assessment. Material was intermediate modulus carbon fibre in a polyurethane resin matrix. Waterjet machine was used to cut the samples at dimensions of $177.8 \mathrm{~mm}$ long and $38.1 \mathrm{~mm}$ wide (no notch). Thickness was $1.86 \mathrm{~mm}$ and area of cross section was $71 \mathrm{~mm}^{2}$. Mean stress $\sigma_{m}=275 \mathrm{MPa}$, amplitude $\sigma_{a}=55 \mathrm{MPa}$ and $\boldsymbol{R}$ was 0.63 . Hence $\boldsymbol{\sigma}_{\max }=336 \mathrm{MPa}$ and $\boldsymbol{\sigma}_{\min }=212 \mathrm{MPa}$. Corresponding testing loads were $\boldsymbol{P}_{\boldsymbol{m a x}}=24 \mathrm{kN}, \boldsymbol{P}_{\boldsymbol{m} \boldsymbol{i n}}=15.2 \mathrm{kN}, \boldsymbol{P}_{\boldsymbol{m}}=19.6 \mathrm{kN}$ and $\boldsymbol{P}_{\boldsymbol{a}}=4.4 \mathrm{kN}$ (tension - tension loading). Referring to published properties of similar material [93], Figure 3.2, the maximum applied fatigue stress throughout the experiment (336 MPa), was below the endurance limits. Therefore fatigue failure was not likely expected to occur before $1 \mathrm{E}+07$ cycles. The numbers of fatigue cycles tested were $4 \%, 8 \%, 12 \%$ and $55 \%$ of cycles to failure. 


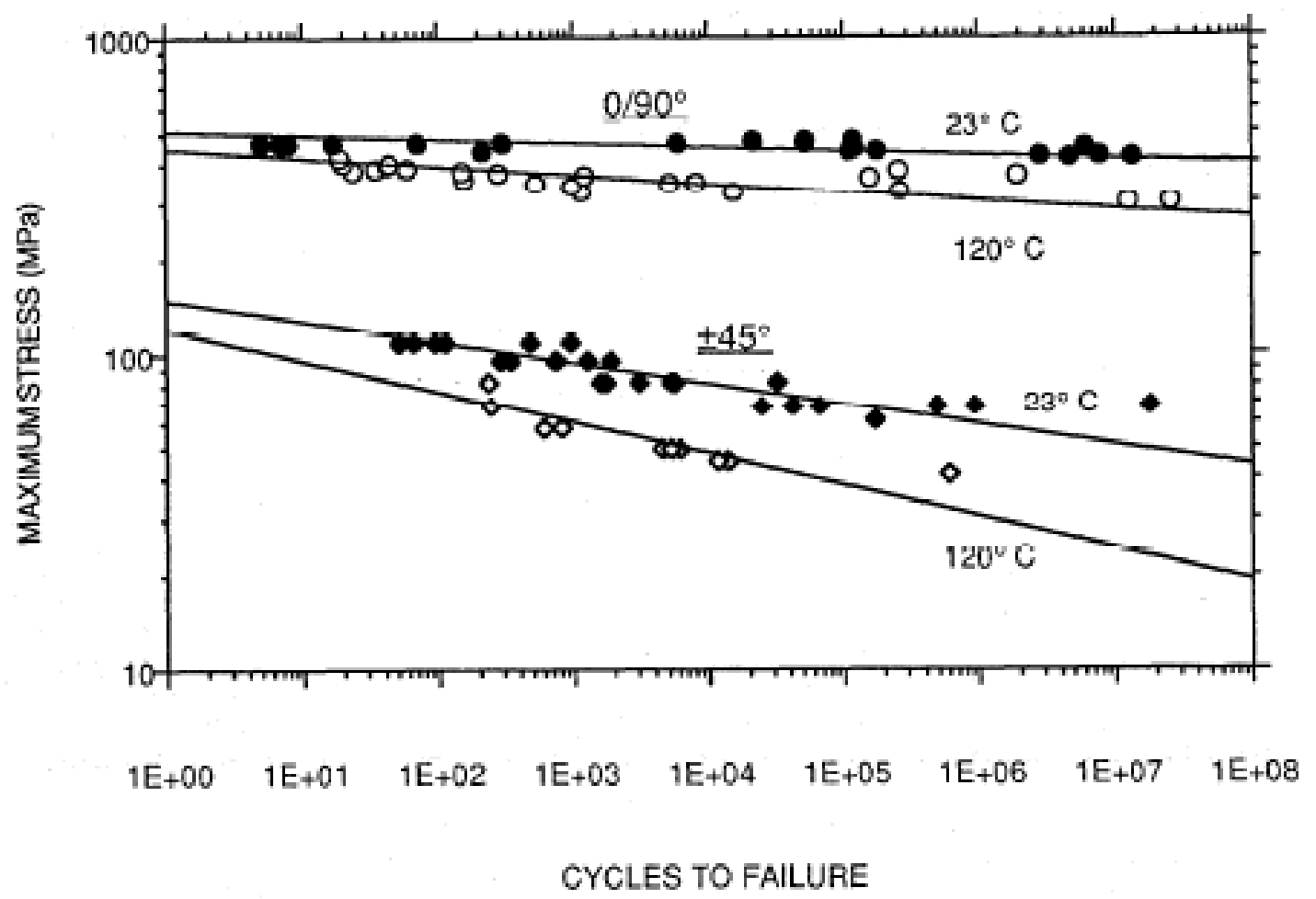

Figure 3.2: Published fatigue curves of $\pm 45^{\circ}$ and $0 / 90^{\circ}$ laminates [93]

\subsubsection{Rendered Volume Analysis}

The 3D volume reconstructed image of the first sample which was subjected to 400,000 fatigue cycles. To estimate the damage caused by cutting and processing of the sample, Scanning Electron Microscope (SEM) images were captured of the sample cut edge and zoomed, Figure 3.3. Images showed delamination occurrences at the edge end, which were attributed to cutting by waterjet machine. For emphasizing the effect of the cutting process, an image of the cross-section in the sample body was compared to an image of the cut edge, Figure 3.4, which clearly featured irregularities and flaws at the cut edge. A VOI of the 3D rendered volume is shown in Figure 3.5. Statistical analysis of density mapping was used to evaluate the material porosity in terms of ratio of voids volume relative to the volume of solid matrix. The calculated porosity was $3.96 \%$. 


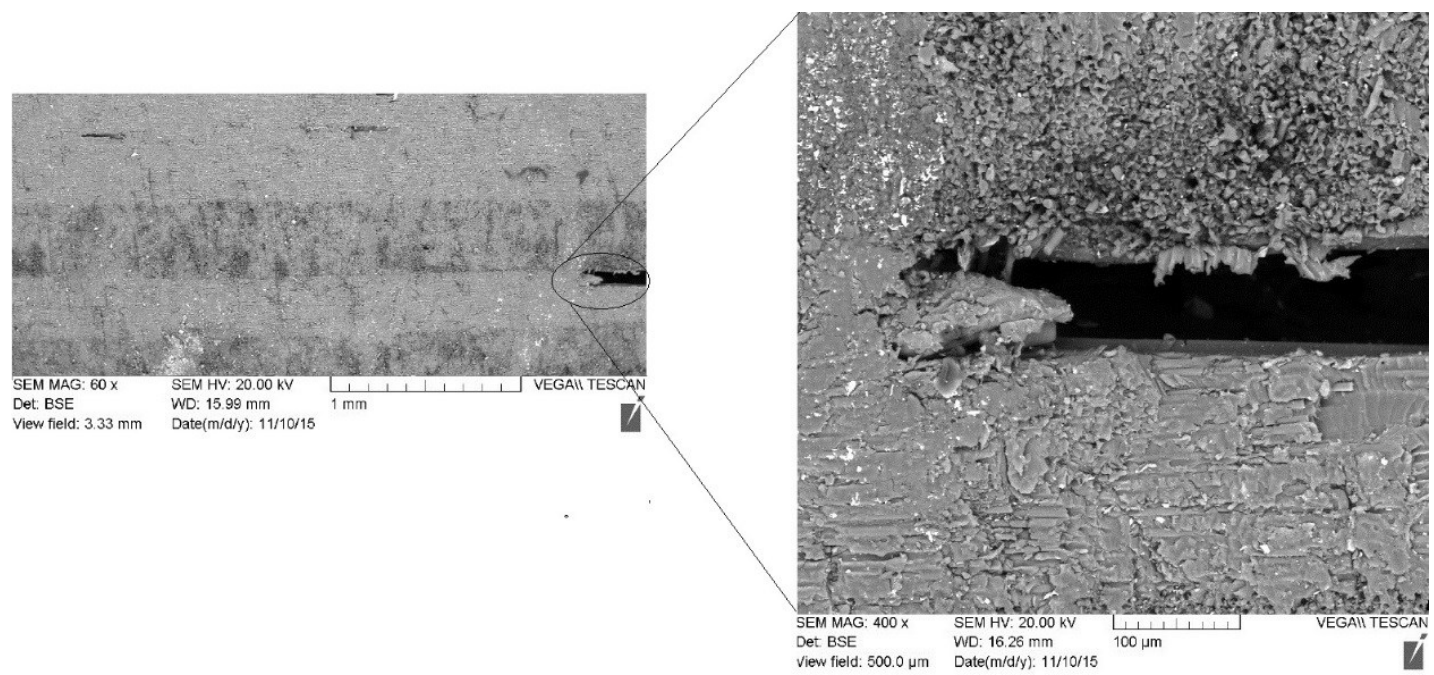

Figure 3.3: SEM image of sample edge showing delamination

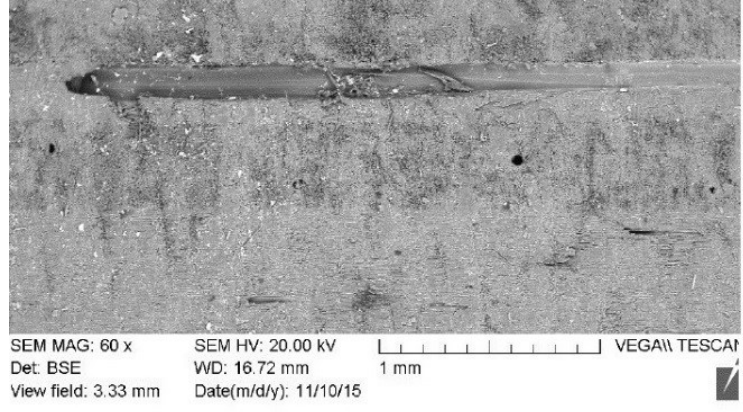

(a)

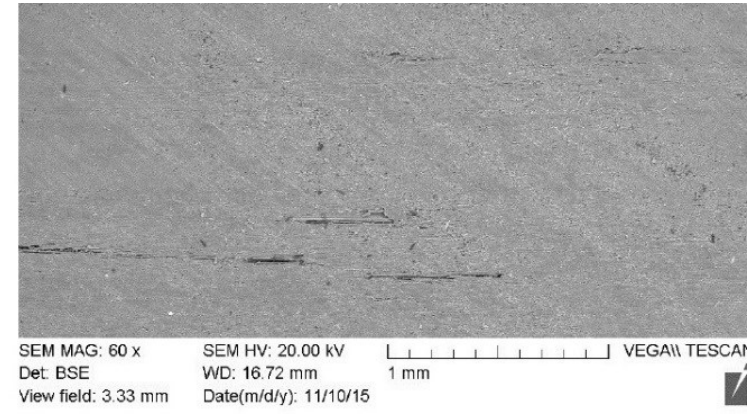

(b)

Figure 3.4: SEM images comparing the cut edge (a) to a cross-section (b) of a sample

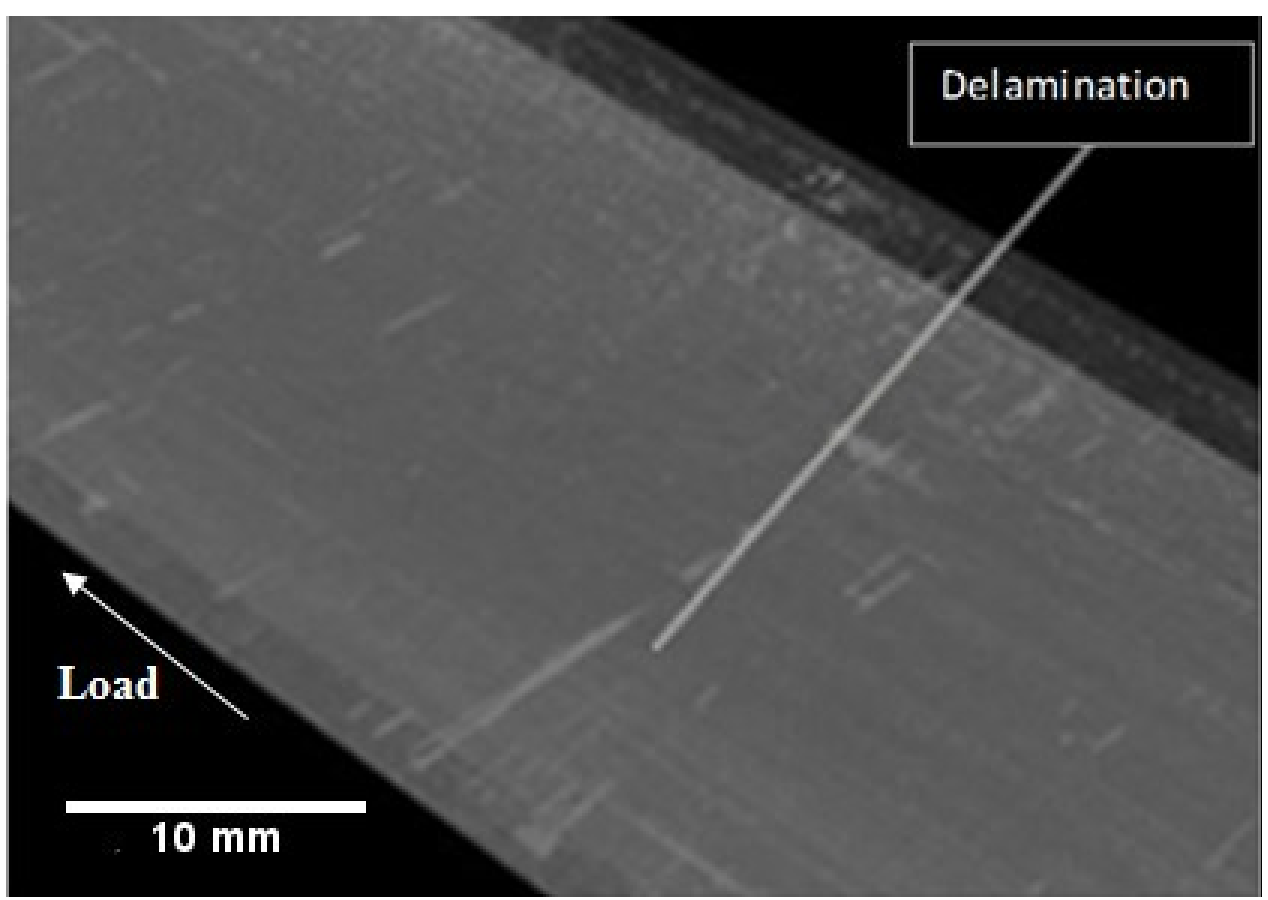

Figure 3.5: $3 D$ volume rendered VOI of CFRP subjected to 400,000 fatigue cycles 
Scanning through the thickness was more detailed by cutting virtual slices at $19 \%, 35 \%$, $54 \%, 67 \%$ and $81 \%$ across the sample thickness, Figure 3.6. It was clear that delamination effects were not likely to occur in the middle plies at that fatigue level of 400,000 cycles, whereas matrix cracks were more dominant near the edges, where the stress concentration is higher due to cutting and processing. Mild matrix cracks were initiated near the edges and in upper and lower plies but fibre breakage and interfacial sliding were not significant.

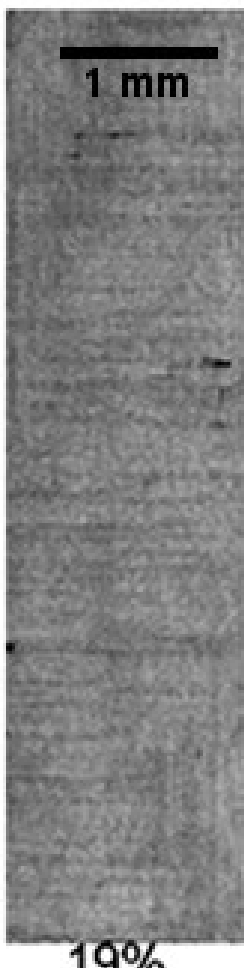

$19 \%$

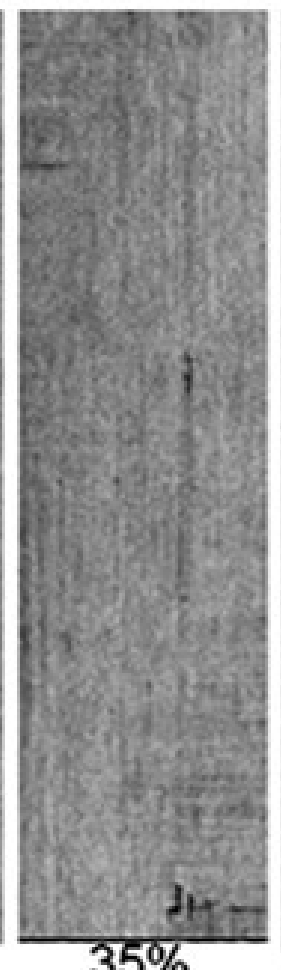

$35 \%$

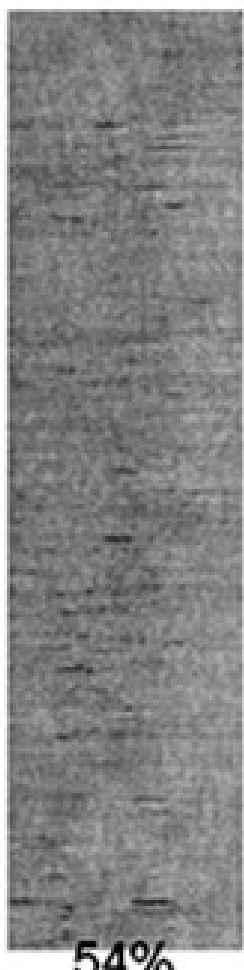

$54 \%$
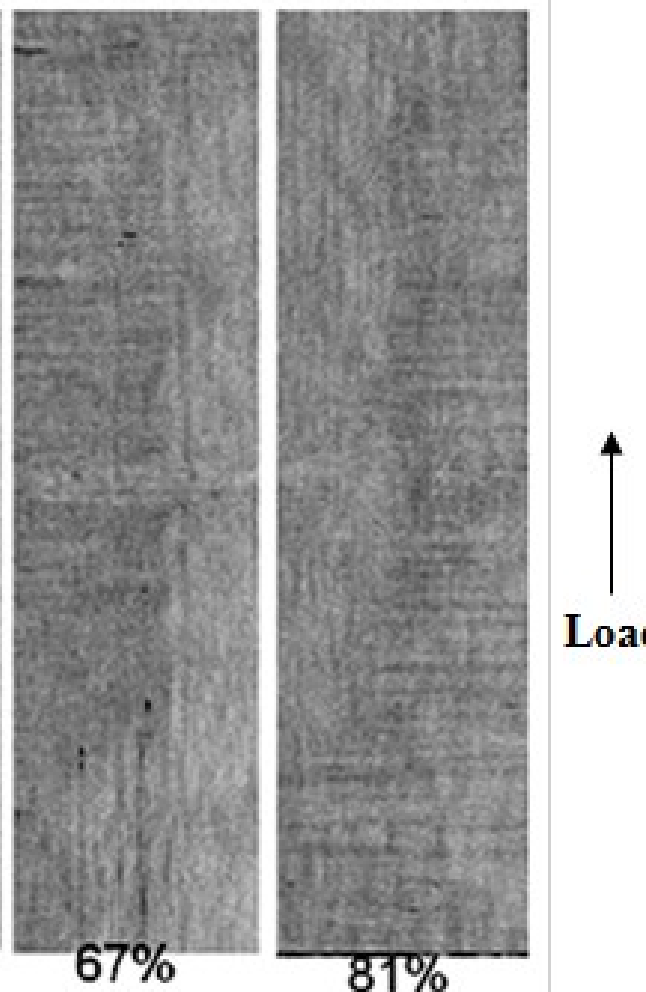

Load

Figure 3.6: Virtual slices across CFRP thickness subjected to 400,000 fatigue cycles

The next sample was subjected to 800,000 fatigue cycles. By investigating the $3 \mathrm{D}$ volume rendered image, shown in Figure 3.7, it was observed that the damage level was slightly increased with a small change in porosity to $3.94 \%$. A cluster of fibre breaks was observed near the edge; a quality-related question arises as to the occurrence of fibre rupture at that low fatigue range, since fibres are the load-bearing component and the relative matrix/fibre properties imply that the matrix was far more susceptible to loads than the fibres. As 
expected, interfacial sliding was observed in directions parallel to the fibre orientations in both $0^{\circ}$ and $90^{\circ}$ plies.

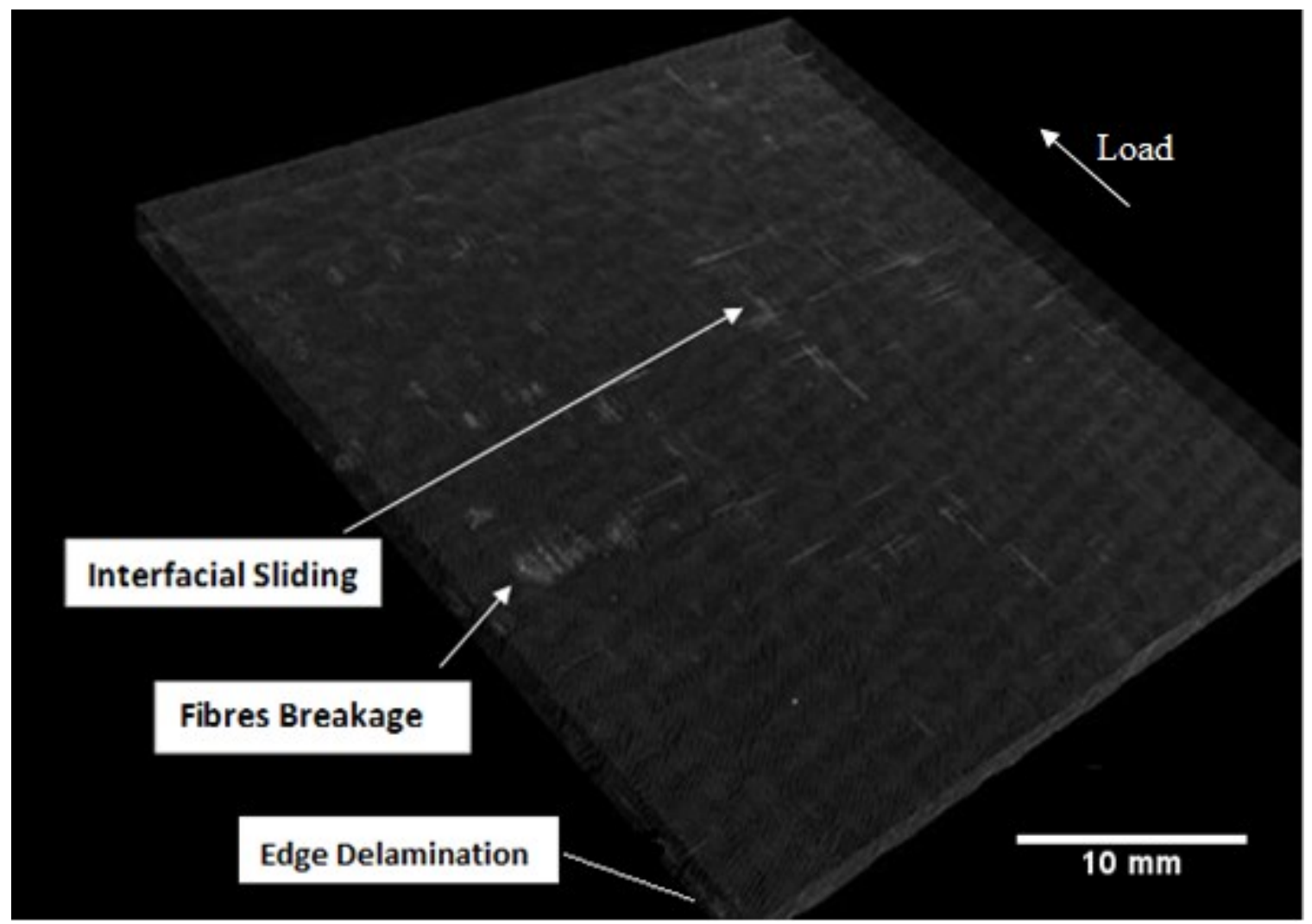

Figure 3.7: 3D volume rendered image of CFRP subjected to 800,000 fatigue cycles

An increased number of fatigue cycles to $1.2 \times 10^{6}$ cycles on the next sample raised the damage level and the porosity changed to $3.90 \%$, as been statically calculated from the density mapping of the images. As seen in the 3D volume rendered image, Figure 3.8, delamination increased significantly in both $0^{\circ}$ and $90^{\circ}$ plies, along with interfacial fibre/ matrix slippage and matrix cracks. A considerable amount of fibre breaks were also observed in the areas near the vicinity of fibre/matrix slippage proving that slippage between fibres and matrix induces weakness to the fibres hence leading to breakage. At 
this damage range, it was desirable to separate damaged areas from the material, Figure 3.9, to analyze damage mechanisms and their distribution in 3D.

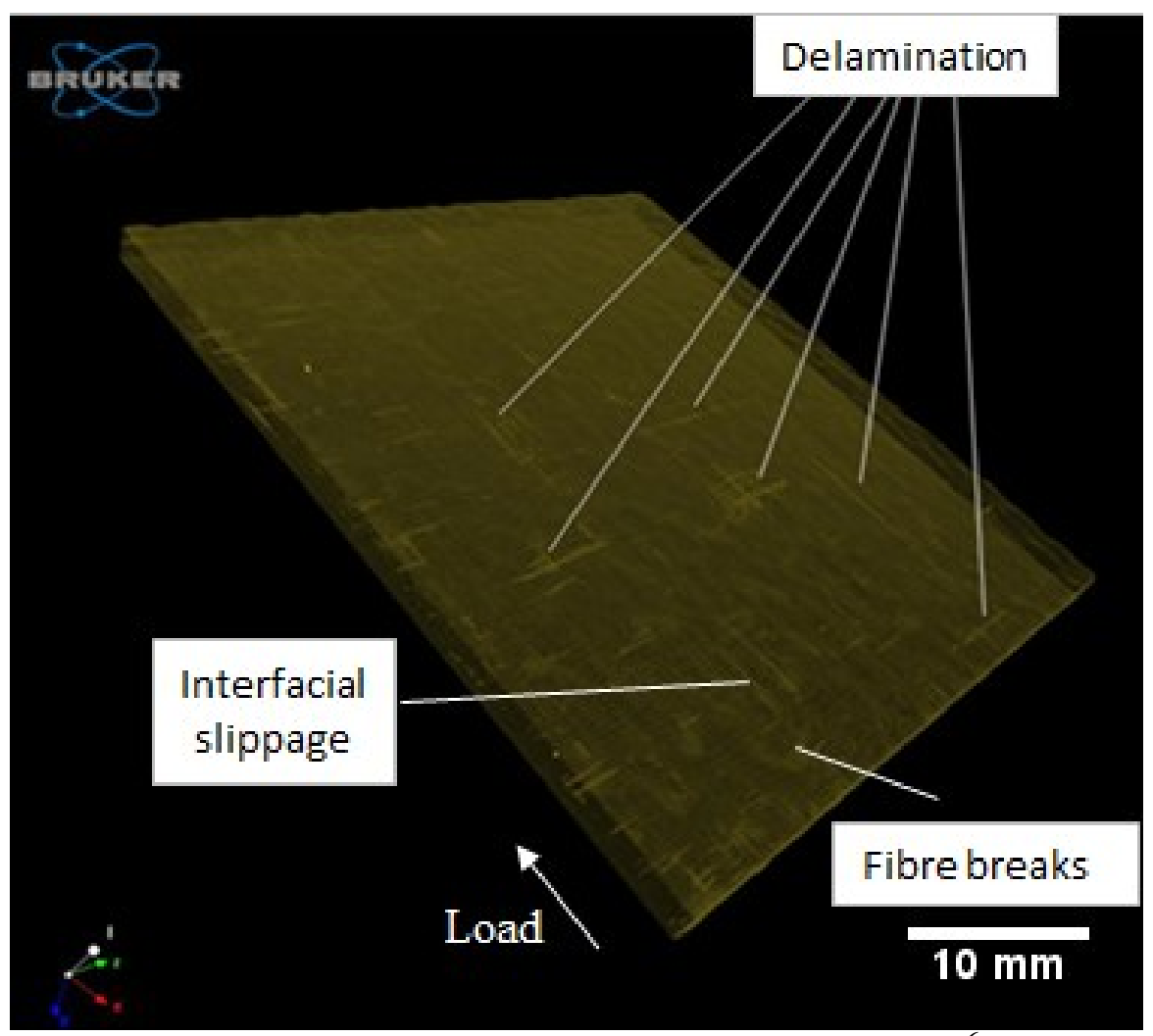

Figure 3.8: $3 D$ volume rendered image of CFRP subjected to $1.2 \times 10^{6}$ fatigue cycles

As seen in Figure 3.9, damage due to more than one mechanism has accumulated in the middle area which was expected to experience evolution of fatigue damage in the absence of acute geometrical alterations and hence no stress concentrations applied. As seen in previous images, delamination was seen at the edges, as a result of waterjet cutting, while interfacial (fibre/ matrix) slippage relative to the degree of adhesion between fibres and matrix was observed through thickness. Micro-cracks were observed followed by the fibre breakage which was an indication of impending failure. At this fatigue level, of $1.2 \times 10^{6}$ cycles, significant fibre breaks were not observed. Rather, there was a high density of micro-cracks distributed over the sample in both $0^{\circ}$ and $90^{\circ}$ directions. 
This agreed with many studies that suggested intra-laminar matrix micro-cracking is the initial damage mode and that the matrix crack density grows and the cracks join together at ply interfaces to create delamination [86-90].

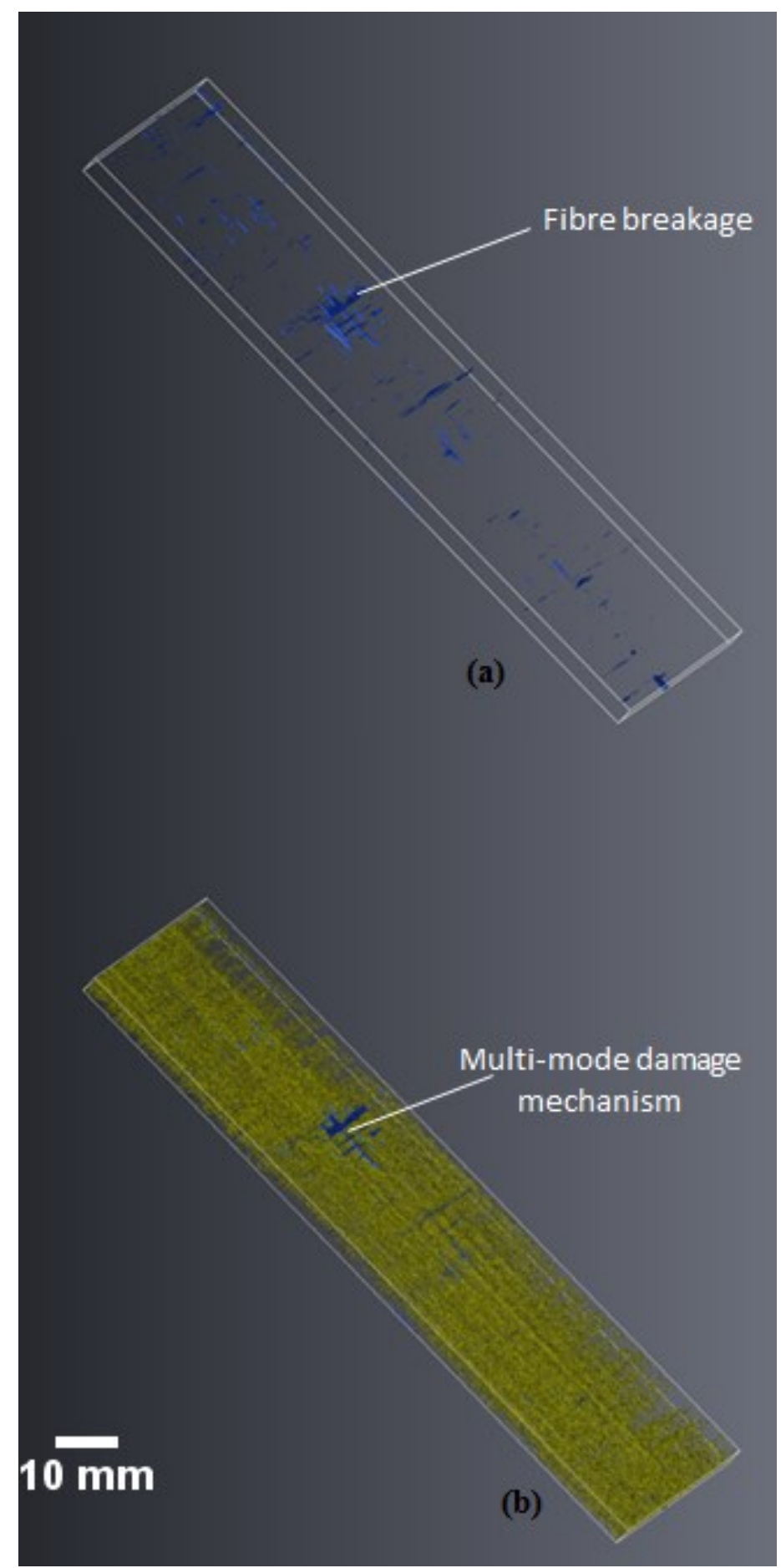

Figure 3.9: Separate damaged areas (a) and damaged material (b) of CFRP sample subjected to $1.2 \times 10^{6}$ fatigue cycles 
Finally, the last sample was subjected to an increased number of $5.5 \times 10^{6}$ fatigue cycles to carry the fatigue damage to a range close to failure, Figure 3.10 . It had a porosity of $3.36 \%$ contradicting the previous linear trend of change in porosity relative to number of fatigue cycles. The 3D volume rendered image shown in Figure 3.10 showed clear broken clusters of fibres heavily distributed at close distances at all levels of thickness. It indicates that the material is experiencing an advanced and multi-mode damage accumulation resulting from complex interactions of the above mentioned damage mechanisms that were detected at earlier stages. Virtual slices at three thicknesses identifying the damage mechanisms and distributions are shown in Figure 3.11 to Figure 3.13.

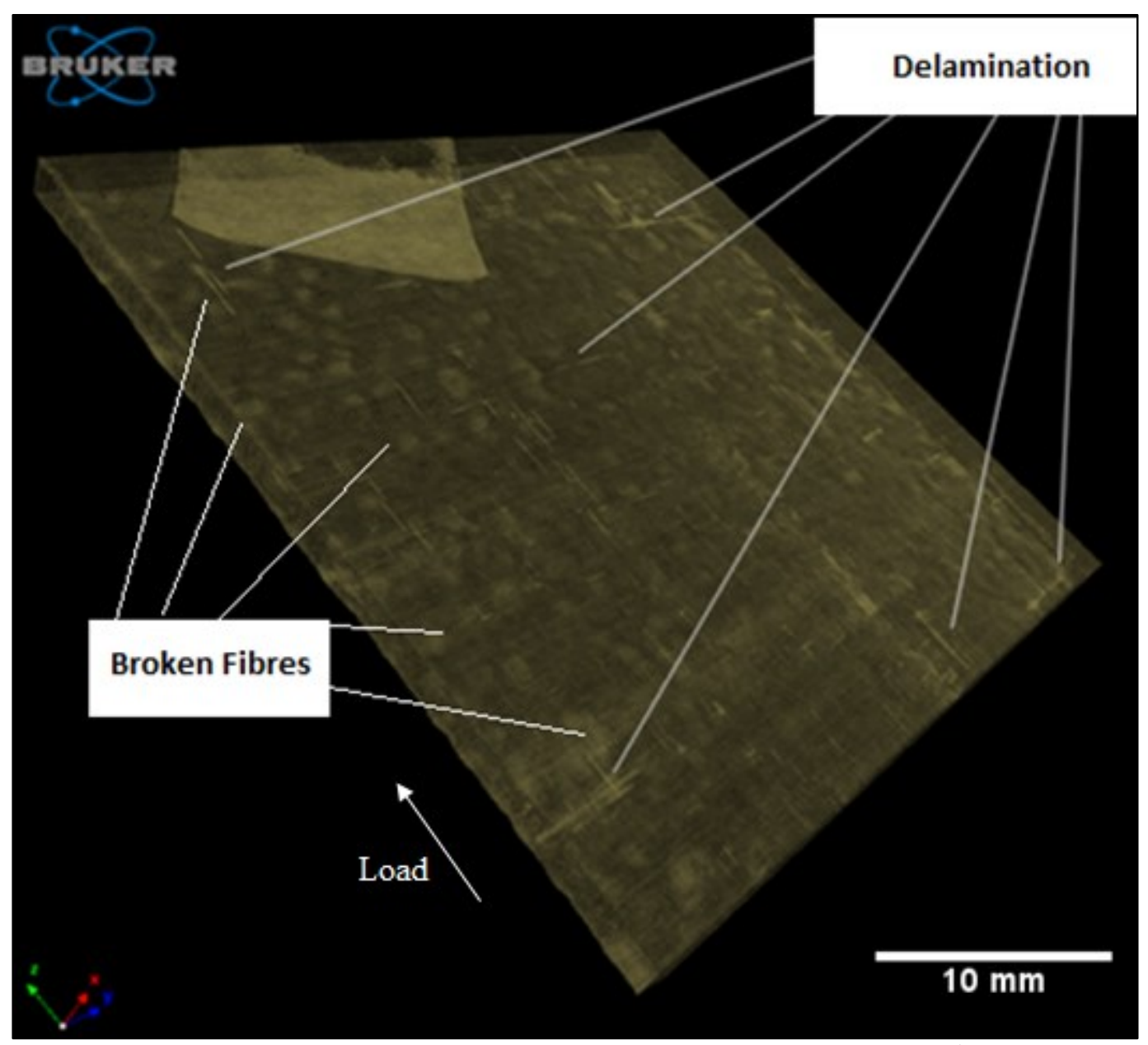

Figure 3.10: $3 D$ volume rendered image of CFRP subjected to $5.5 \times 10^{6}$ fatigue cycles 
Delamination was observed at a wide scale at different thicknesses especially near the edges. It was relevant to the manufacturing technique, cutting process by using the waterjet and the pre-occurrence of defects or voids. Interfacial slippage was detected in all levels of thickness and in middle areas. Interfacial slippage was distinguished from delamination owing to the difference in length, which is expected to be more in delamination, and due to orientation of cracks relative to the fibre direction. Fibre breakage appears to initiate near the edges then propagate to the middle areas.

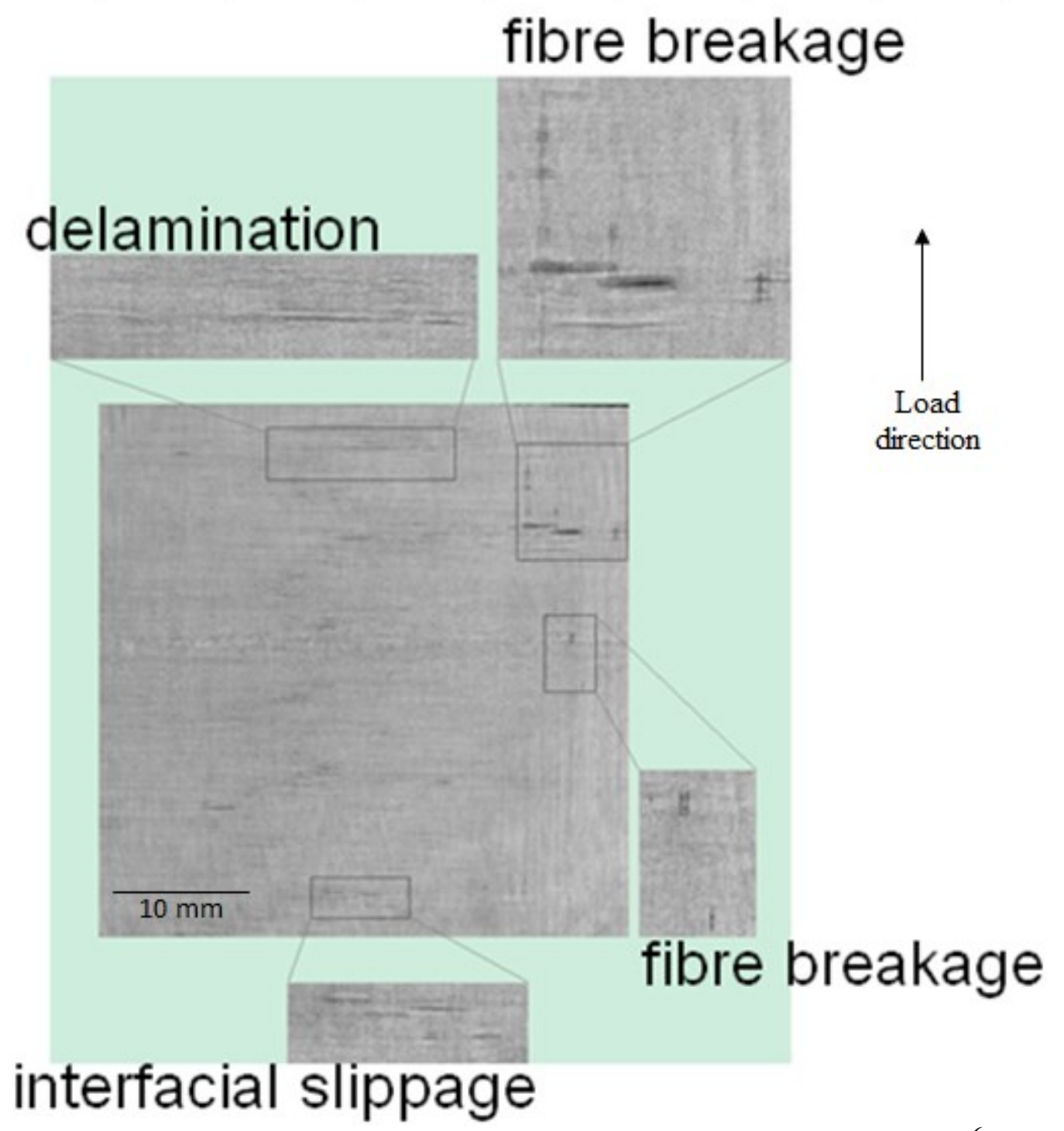

Figure 3.11: Virtual slices through 25\% thickness of CFRP subjected to $5.5 \times 10^{6}$ cycles 


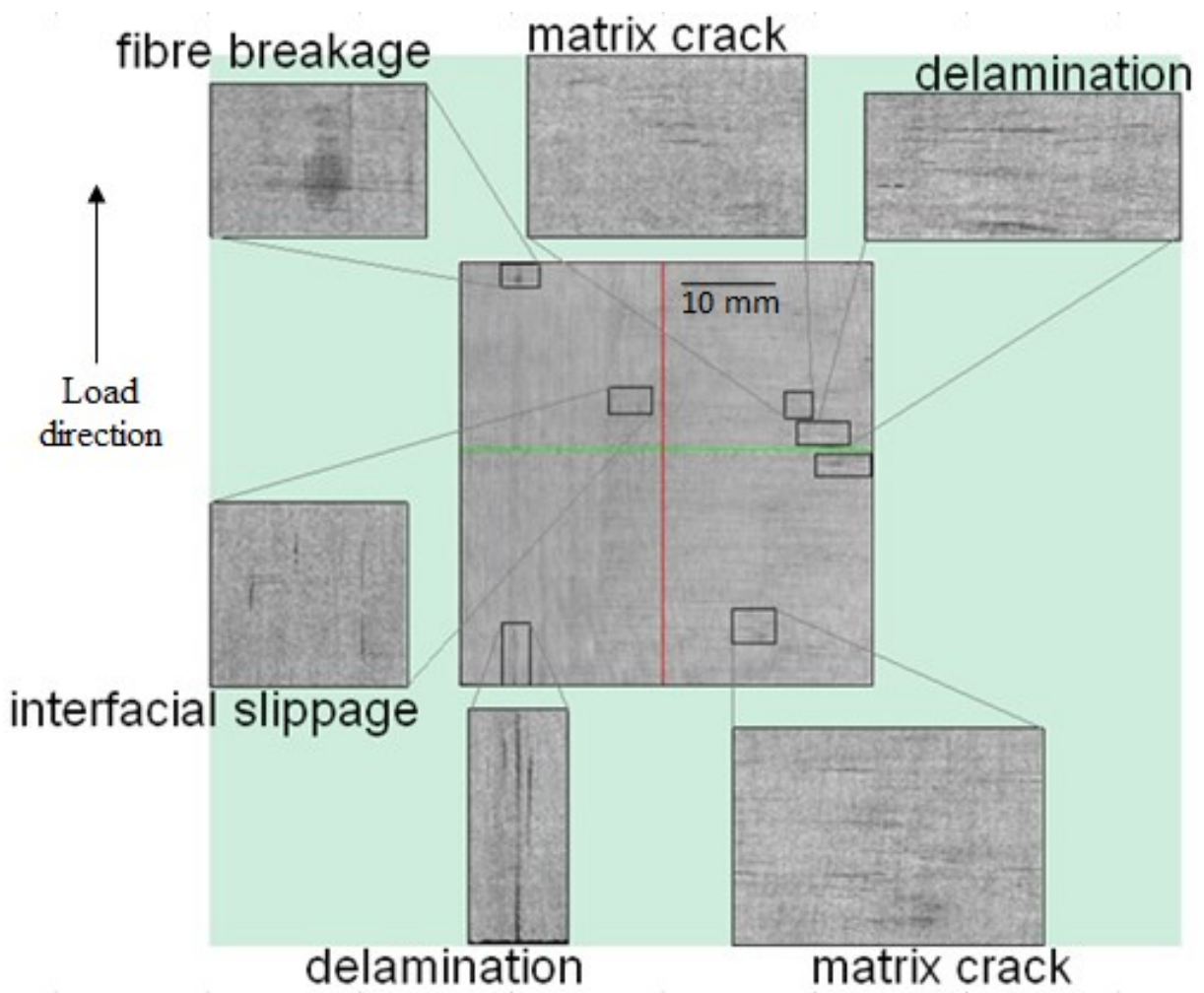

Figure 3.12: Virtual slices through 50\% thickness of CFRP subjected to $5.5 \times 10^{6}$ cycles

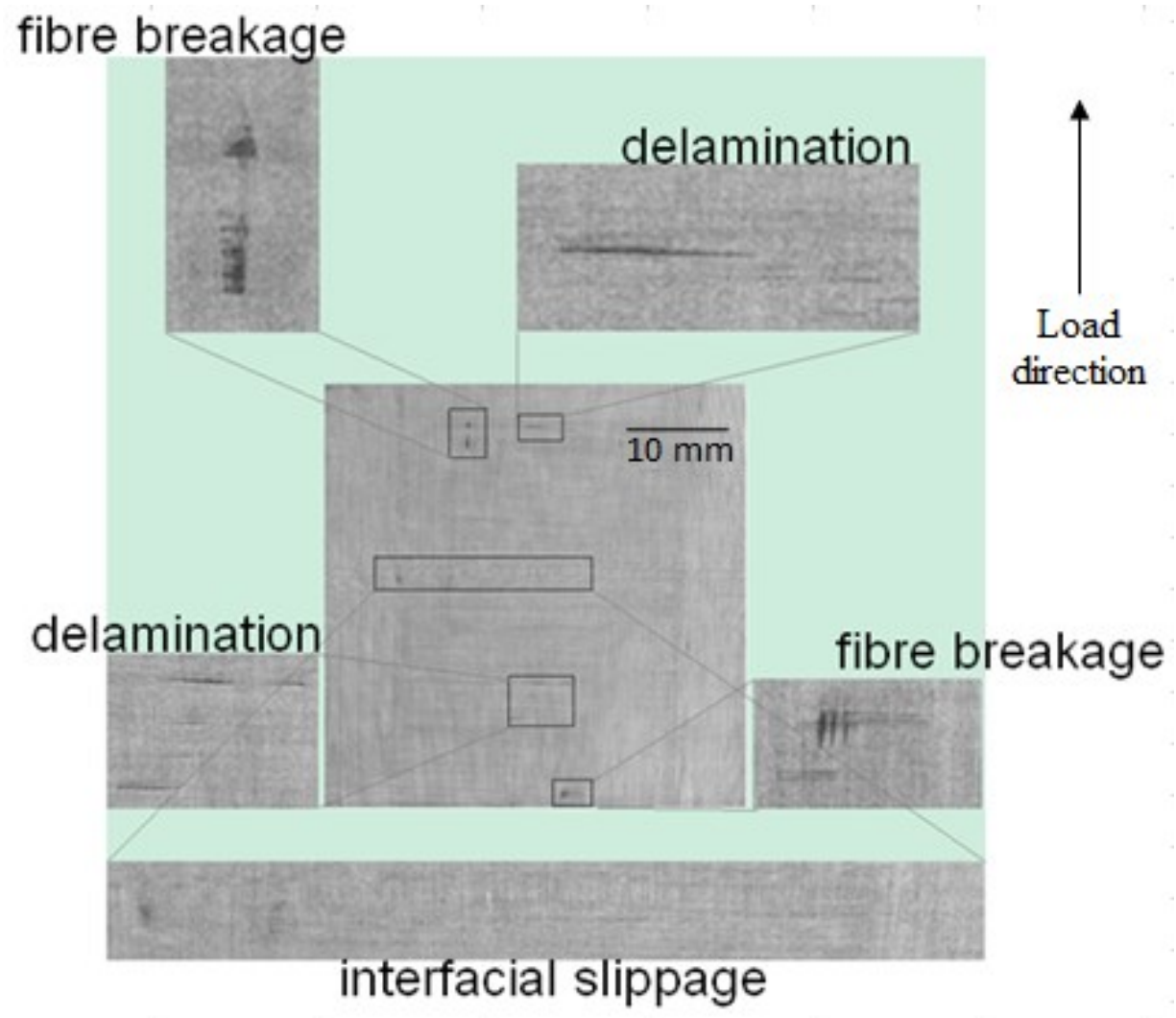

Figure 3.13: Virtual slices through 75\% thickness of CFRP subjected to $5.5 \times 10^{6}$ cycles 


\subsubsection{Conclusion}

Qualitative characterization of damage propagation of laminated CFRP is a major step to enabling the modelling and simulation of fatigue failure mechanisms using FEA tools. A major limitation is experienced with conventional methods of defining failure criteria that are available in literature and are widely implemented for simulating failure scenarios using FEA and analytical design software. That is; these criteria can only predict the final event of damage at the macroscopic scale and they have a limited capability for predicting the damage mechanisms and the criteria leading to the early evolution of cracks at the microscopic scale as explained by [7].

Data obtained from visualization of X-ray microCT 3D images were used to identify damage mechanisms, namely delamination, interfacial debonding (separation between fibres and matrix), interfacial slippage and fibre breakage. It was shown that damage initiates with delamination near the edges in the absence of stress concentrations.

Propagation featured slippage in the vicinity of the delaminated area, then the weak matrix cracked before fibre breakage finally brought the material close to the dramatic failure. Strain was recorded in longitudinal dimension using an extensometer, resulting in an average reading of 0.018 .

Material porosity tended to change by $0.01 \%-0.02 \%$ every 100,000 fatigue cycles, according to density mapping analysis obtained from CTAnalyzer software [66]. Results showed inconclusive very slight change; hence motivated a more thorough study of density distribution throughout the separate damaged surface. Density mapping will be discussed in more details in Chapter 6 of this work. 


\subsection{Proposed Procedure of Simulating Fatigue Damage by Using MicroCT Scans in Abaqus}

Commercial FEA tools are widely employed for modeling fatigue damage propagation in materials subjected to fatigue loading. Arguments were raised regarding their reliability in this context due to neglecting the stress strain redistribution at the crack tip which requires continuous remeshing. Alternatively, other techniques were introduced to overcome this shortcoming without the need to remesh at the crack tip [94]. The commercial Abaqus FEA package incorporates this new technique by implementing either Virtual Crack Closure Technique (VCCT) based on LEFM concepts [95]. However, application of VCCT in available commercial tools typically pertains only to 2D quasi-static problems.

Using Abaqus with 3D imaging was successfully introduced in literature [96, 97]. Abaqus FEA simulations were described in literature for simulating damage in different composite materials as being compared to experimental data; on the one hand for developing physical relations by implementing approaches of fracture mechanics $[98,99]$, or on the other hand, for assessing the accuracy of the recently developed approaches [100, 101].

In this section, an assessment was performed on the reliability of Abaqus tool in addressing combined-mode 3D damage propagation caused by fatigue stress, and results from simulations were compared to experimental data. Virtual slices obtained throughout the thicknesses of the samples volumes were visually analyzed and further rendered and processed by applying a threshold method to define the 3D damaged areas and classify the damage modes. Finally, the stacked segmented volumes were meshed for importing to FEA modeling. Limitations of the available commercial FEA tool in addressing the problem 
were discussed, and the introduced testing procedure was proved to be reliable in providing the quantitative information necessary for analyzing fatigue behaviour. The implemented procedure was introduced in CAMX Conference [20].

\subsubsection{Virtual slicing}

Raw images from microCT scans were imported into DataViewer software [66] to allow virtual slicing in either of the three axes. Sets of images were imported for each of the 4 samples subjected to a different number of fatigue cycles. Size of samples was $38 \mathrm{~mm} \mathrm{x}$ $40 \mathrm{~mm}$ (1854 x 1836 pixels). A volume of interest (VOI) containing a delamination defect was defined, partially appear in Figure 3.14. Its dimensions were 9 mm x 3 mm (400 x 136 pixels) where a stack of 57 consecutive images were saved for further processing.

\subsubsection{Segmentation}

Segmentation is the process of applying a threshold to separate surfaces of different densities or X-ray attenuation. ImageJ [102] was used to apply thresholds to the images. A total of 16 available threshold methods were investigated, and the Yen method [103] was

chosen. A 2D slice of the segmented set of images of the sample subjected to 400,000 cycles using ImageJ [102] Yen method appears in Figure 3.15. By implementing this method, the algorithm was extended to find on which side of the maximum peak the data goes the furthest and searches for the threshold within that largest range.

The stack of segmented sequences was saved for the next process; that is meshing. It is worth mentioning that ScanIP software environment [104] has a built in threshold tool. However, the upper and lower limits should be manually defined based on visual analysis. Lower and upper limits of 130 and 255 respectively were defined to obtain a mask of voids 
and another for "FloodFill" surface which represents the cracked matrix. ScanIP software [104] was used to provide a 3D rendered volume of segmented images, Figure 3.16, constructed of the same sample which was segmented by ImageJ [102], Figure 3.15, so as to allow 3D visual detection of voids and cracks. Different void contents were observed by comparing both segments attributed to different threshold methods.

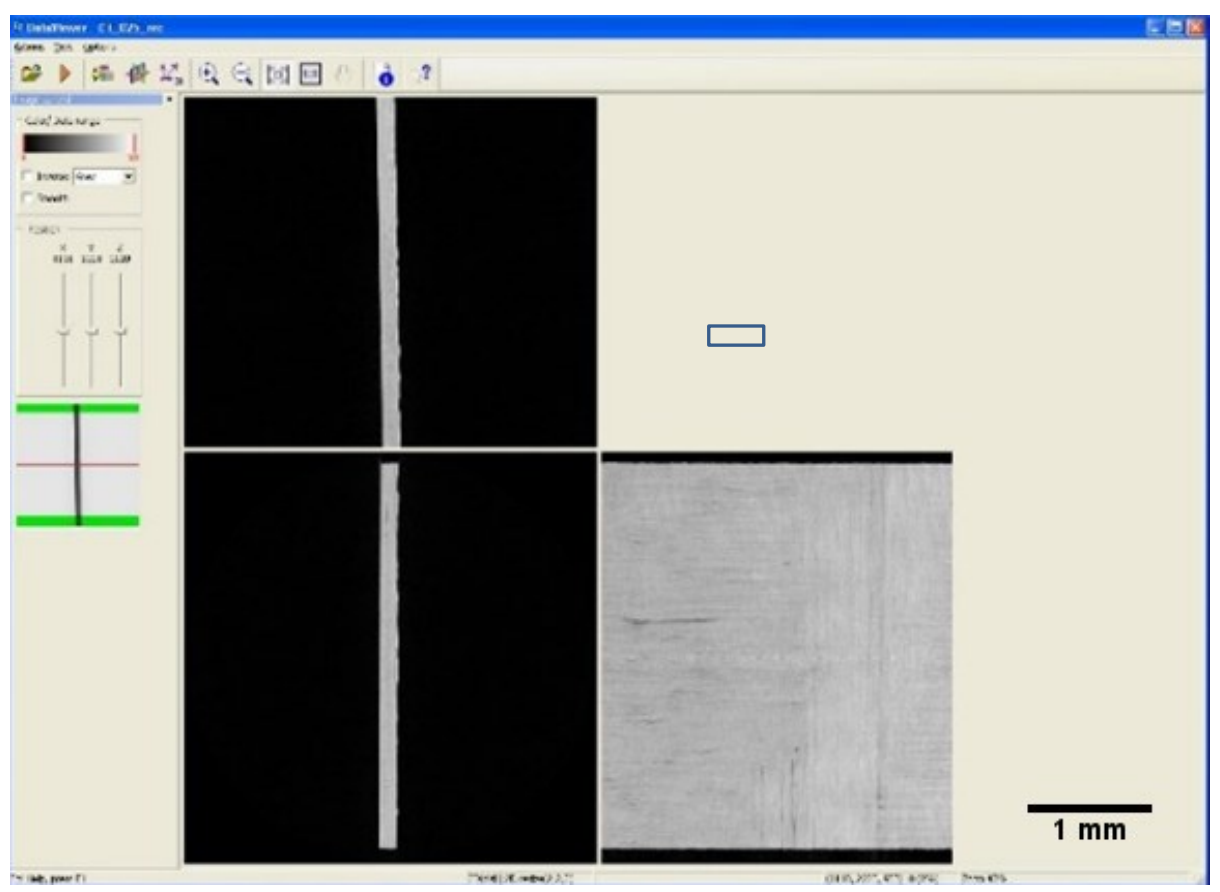

Figure 3.14: DataViewer 58mm x $58 \mathrm{~mm}$ virtual slice through thickness
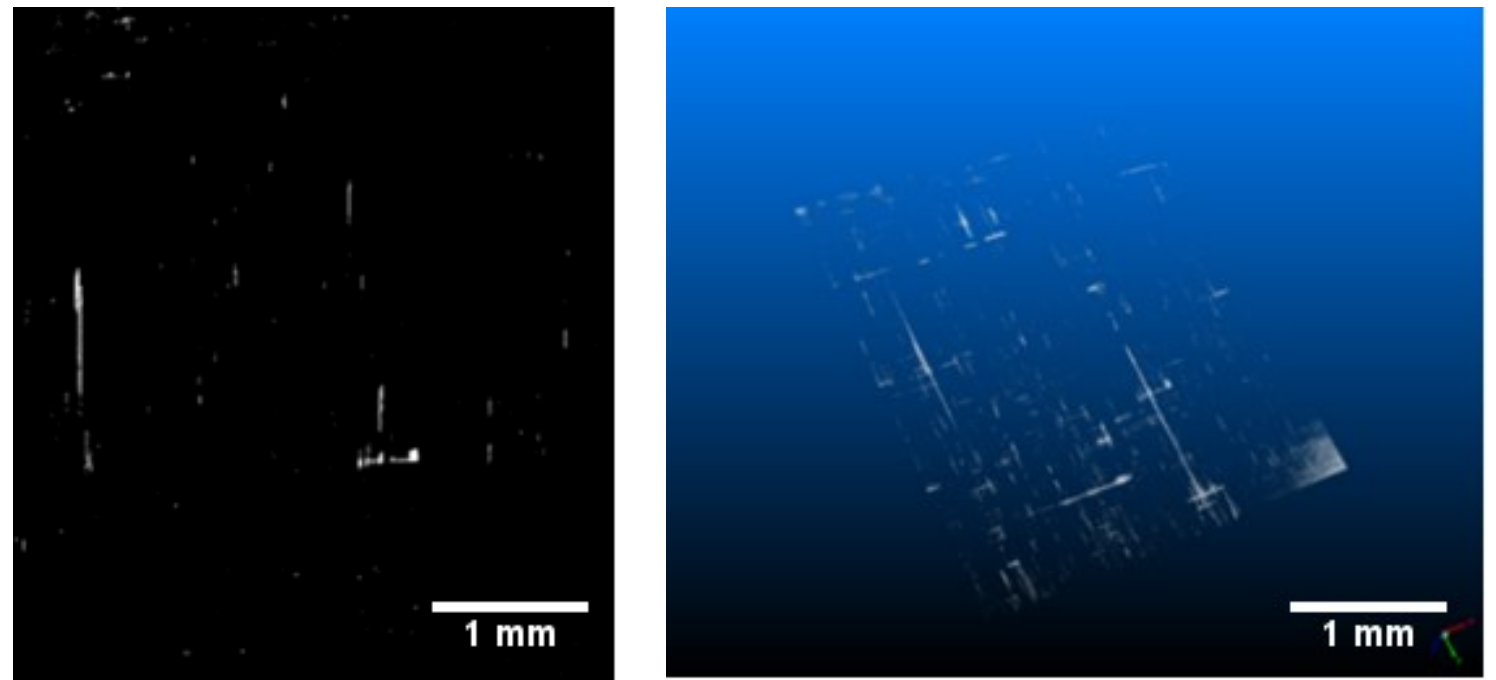

Figure 3.15: ImageJ segmentation

Figure 3.16: 3D segmented volume render - ScanIP 


\subsubsection{D plotting}

The code developed for 3D plotting is an in-house plotting tool developed at Carleton University. This tool imports the virtual slice images of the specimen, produced by DataViewer [66] into MATLAB library and stores them as an image matrix. Since the images imported in MATLAB are gray scale images, thresholding image processing technique was applied to all images in order to highlight the desired features such as cracks and voids.

The image threshold could be adjusted in order to obtain additional features such as fibre orientation and specimen orientation. The code script is attached in Appendix D. The output of one iteration is seen in Figure 3.17. It shows a 3D plot of the void content in the defined VOI. This plot is analogous to the 3D constructed volumes provided by CTVox, but with more flexibility in modifying its capabilities. This code, if expanded may give quantitative information on the location and dimensions of the voids. However, this quantitative analysis is carried out here by using ImageJ software [102].

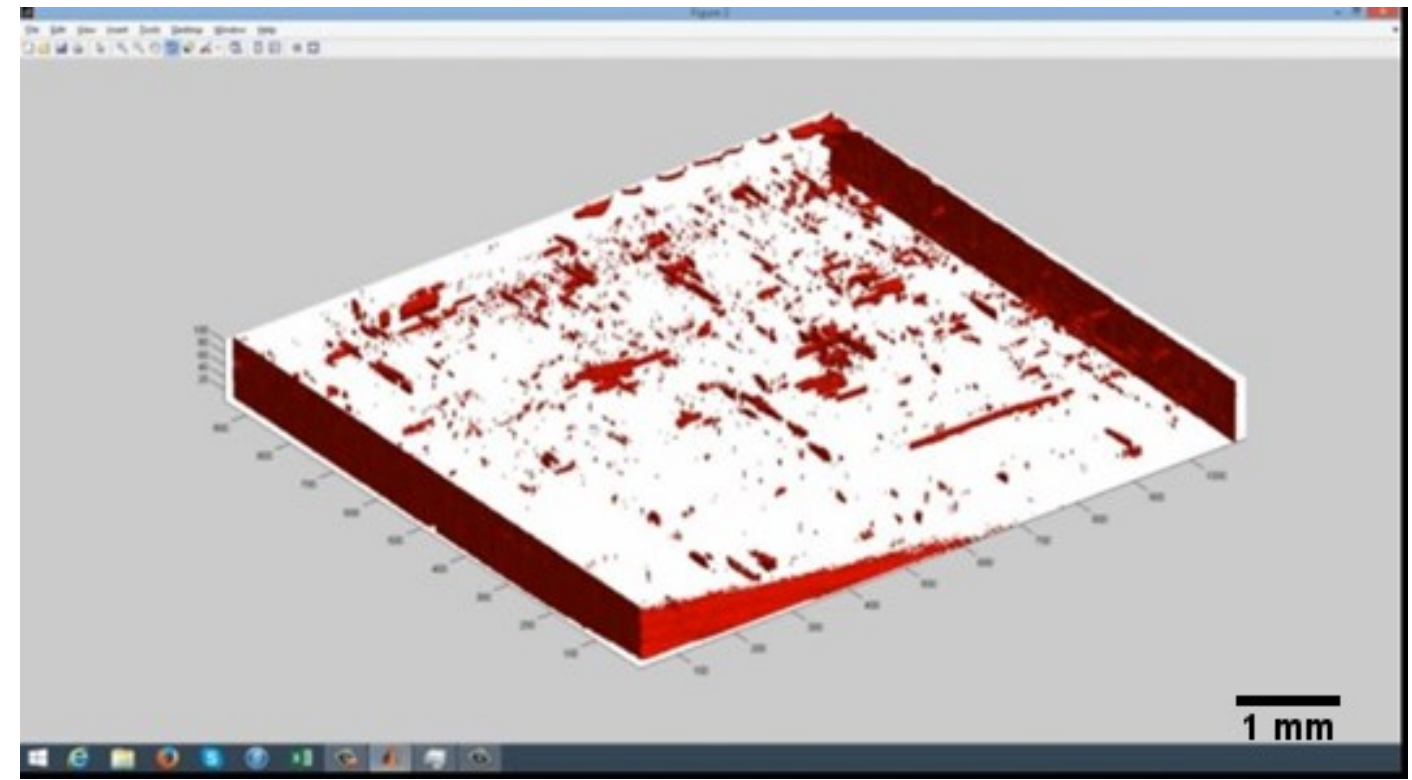

Figure 3.17: 3 D representation of cracks plotted in MATLAB 
Since the images imported are grey scale, the MATLAB code converts these images into black-and-white images. By converting the images from grey scale to black-and-white, the memory space requirement is reduced from 8 bit to 2 bit, resulting in faster post processing time. This conversion to black and white images becomes particularly important as the code connects the points between multiple images in its post processing image matrix and converts them into a solid surface which can then be plotted in 3D in MATLAB.

\subsubsection{Meshing}

Meshing was performed by using 2 techniques; Tetgen which is a sub-tool of Iso2mesh software [105] and ScanIP software environment [104]. Iso2mesh is a MATLAB-based code where a code published in [96] was adapted and used as a guide for stacking the segmented images and applying the mesh function given a predefined element size and number of nodes of a volume of interest, Figure 3.18. However, Tetgen [105] did not prove efficient for providing a refined mesh of highly curved surfaces.

Alternatively, ScanIP software [104] was used to provide a tetrahedral/ hexahedral mesh of the floodfilled mask, Figure 3.19, shows the meshed sample that was subjected to 400,000 fatigue cycles. The .inp file produced was ready to import to Abaqus but the size was 1.5 GB which was not feasible for efficient processing given an 8 GB RAM processor.

To reduce the processing burdens, a VOI was segmented and the $+\mathrm{FE}$ add-on module was used for volumetric Abaqus mesh generation for obtaining a tetrahedral mesh, Figure 3.20, and an .stl triangular mesh for rapid processing, Figure 3.21 Mesh information was as follows: points: 262, mesh tetrahedral: 863, mesh triangles: 1943, mesh sub faces: 434 and mesh sub segments: 635 . Similar files were obtained for the other 3 samples. 


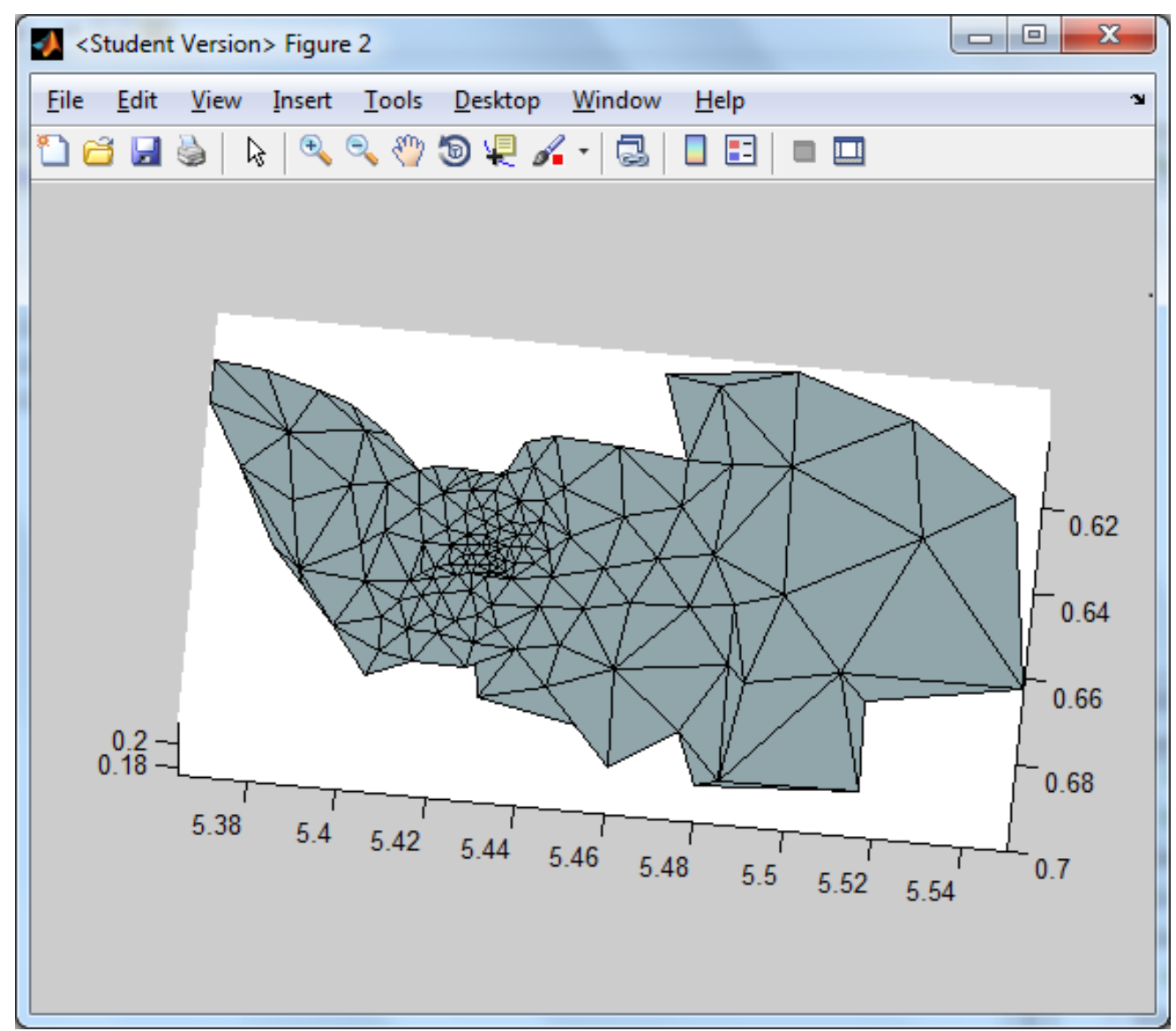

Figure 3.18: Tetgen mesh of a VOI

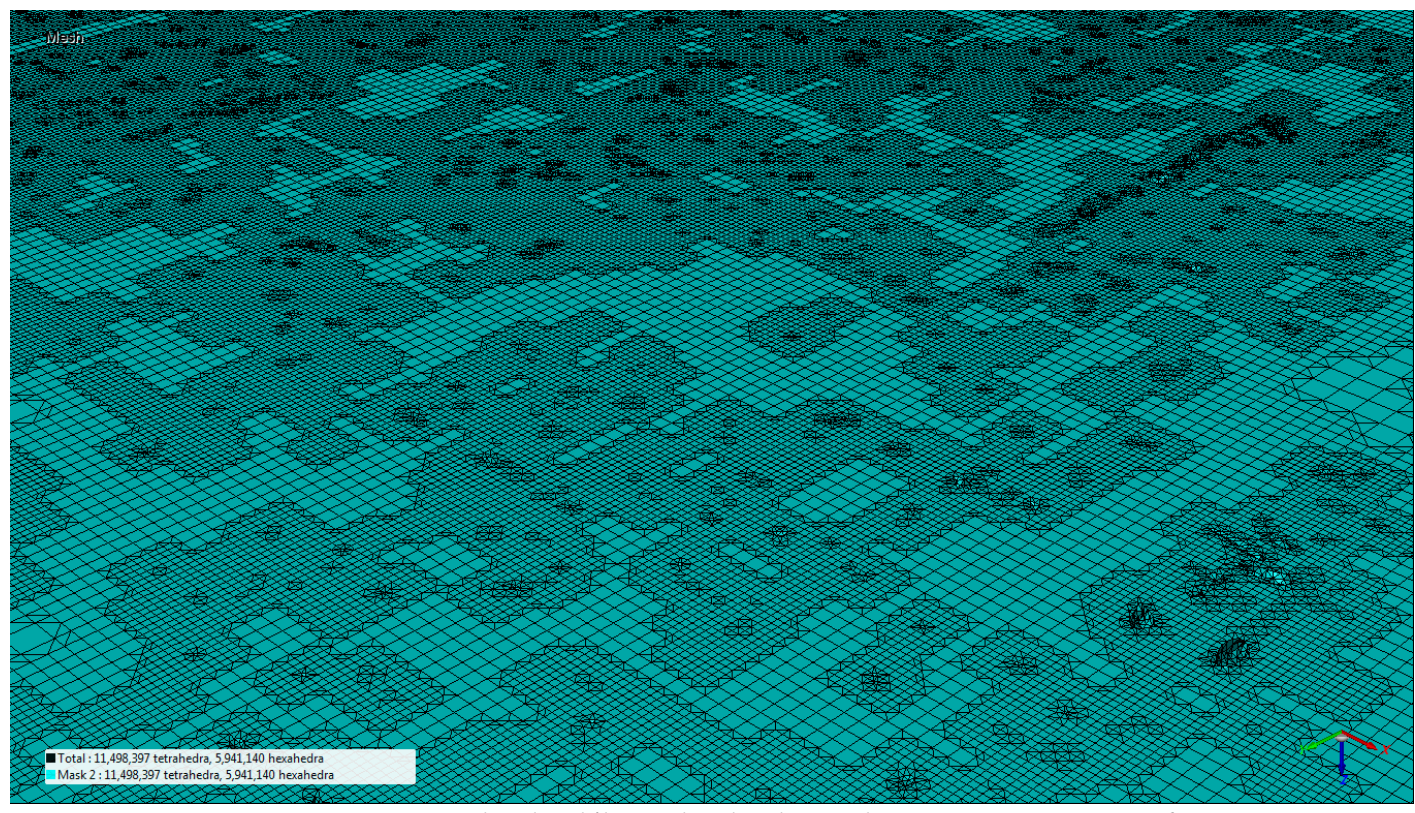

Figure 3.19: Tetrahedral/hexahedral mesh using ScanIP software 


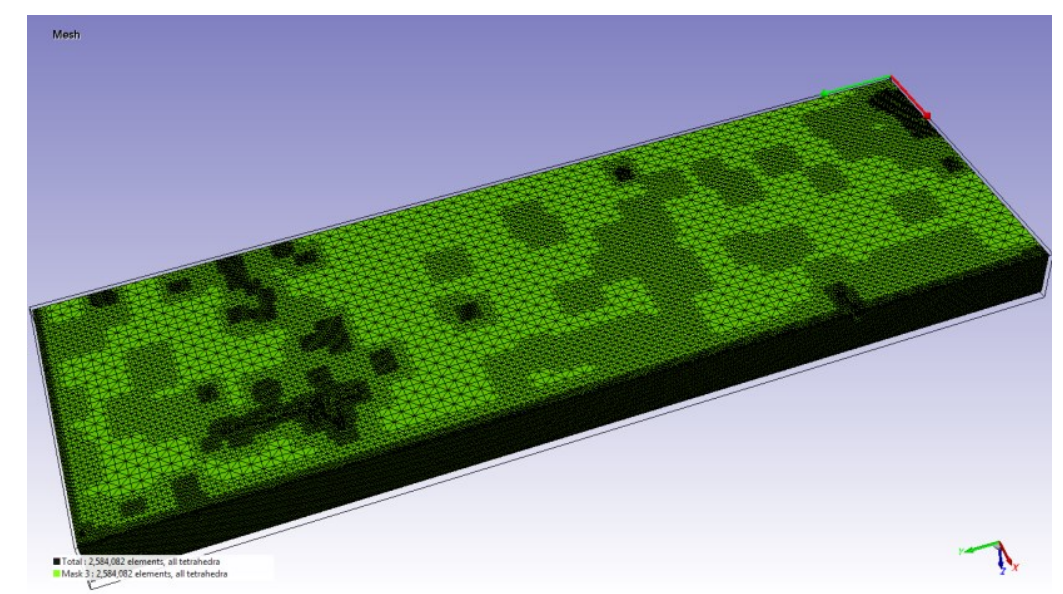

Figure 3.20: A tetrahedral mesh of a VOI using ScanIP software

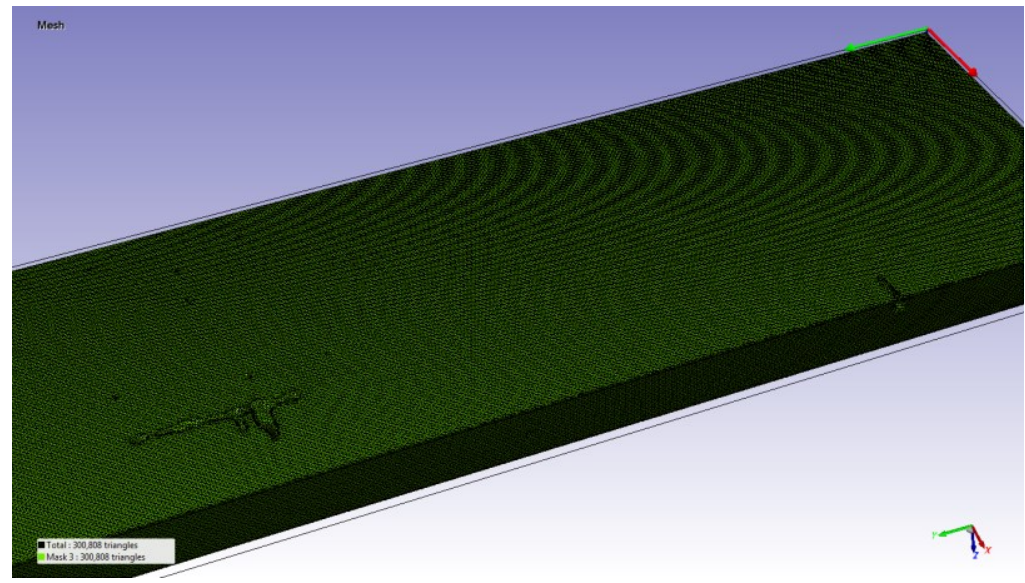

Figure 3.21: A triangular mesh of a VOI using ScanIP software

\subsubsection{Importing into Abaqus}

The .inp file was imported into Abaqus, and an initial crack was defined a priori as obtained from X-ray scan of the sample subjected to 400,000 cycles. The measured crack length was $0.31 \mathrm{~mm}$. A maximum normal stress of $330 \mathrm{MPa}$ was modelled. To simplify the simulation, material was assumed to be microscopically isotropic with Young's modulus of $70 \mathrm{GPa}$ and Poisson's ratio of 0.2 .

For simulation, the coupon was meshed with plane strain elements with 14448 total number of nodes and 14100 total number of elements (linear quadrilateral). Simulating crack propagation following an arbitrary path is tracked in Figures 3.22 and 3.23. 
For running the simulation model, stiffness data published in literature were used; normal strength $\boldsymbol{S}_{\boldsymbol{n}} 50 \mathrm{MPa}$, fracture energy, $\boldsymbol{G}_{\mathrm{n}}$ (normal mode) $=350 \mathrm{~J} / \mathrm{m}^{2}$, in-plane shear strength, $\boldsymbol{S}_{\boldsymbol{s}}=30 \mathrm{MPa}$, fracture energy $\boldsymbol{G}_{\boldsymbol{s}}$ (in-plane shear mode) $=700 \mathrm{~J} / \mathrm{m}^{2}$, out-of-plane shear strength, $\boldsymbol{S}_{\boldsymbol{t}}=30 \mathrm{MPa}$ and fracture energy $\boldsymbol{G}_{\boldsymbol{t}}$ (out-of-plane shear mode) $=700 \mathrm{~J} / \mathrm{m}^{2}[106]$.

Verifying versus microCT experimental outcome, showed that virtual simulations of Abaqus model were 50\% accurate.

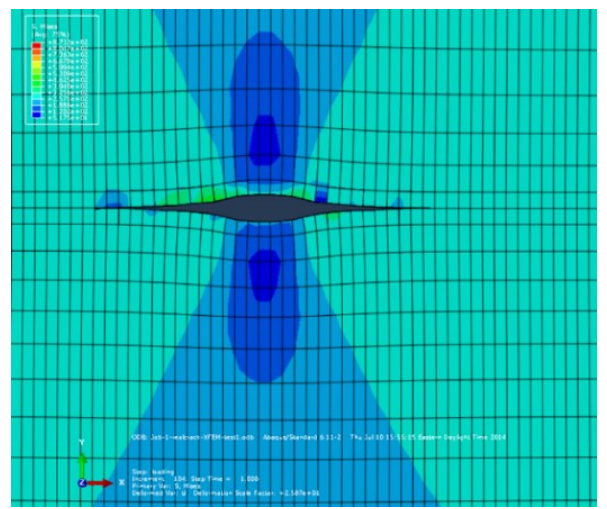

Figure 3.22: Abaqus simulation of 2D static crack obtained from microCT scans
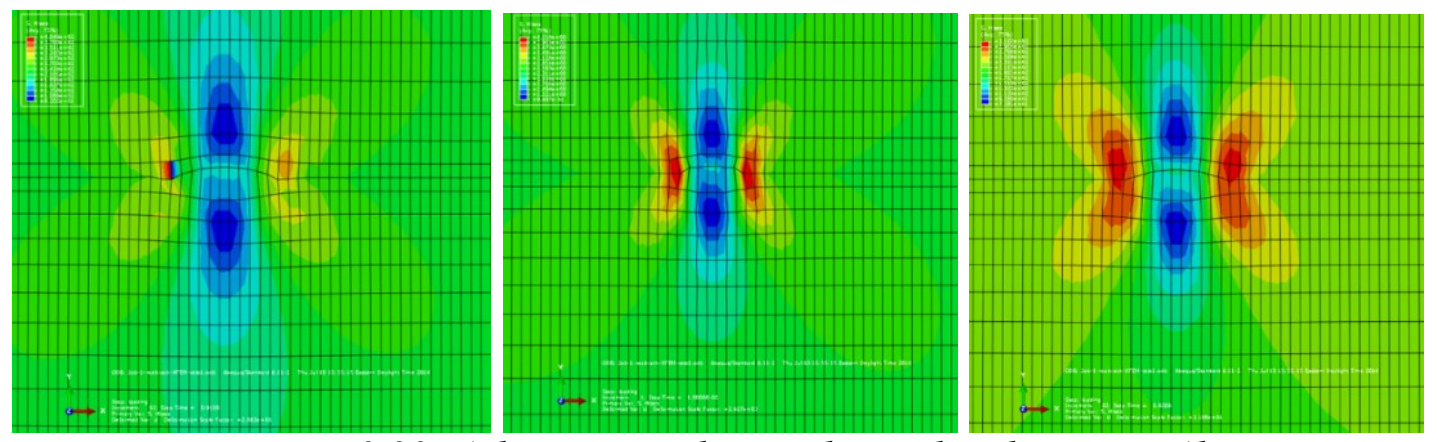

Figure 3.23: Arbitrary crack growth simulated using in Abaqus

\subsubsection{Conclusion}

Simulating fatigue failure in commercial FEA tools requires the creation of a robust algorithm for incorporating microCT scan data into Abaqus and other FEA tools. An inhouse solution was developed using MATLAB-based tools and available commercial software. However, the former did not prove its reliability with regards to mesh refinement 
with CFRP material characterized by numerous defects having complicated geometry and acute curved surfaces. Alternative integrated software tools provided improved solutions for the segmentation and meshing of such surfaces. Combining multiple tools allowed for the extraction of quantitative information in terms of defining the onset of cracks and their initial dimensions and geometry. A flowchart demonstrating proposed tools for exporting microCT scans to Abaqus appears in Figure 3.24.

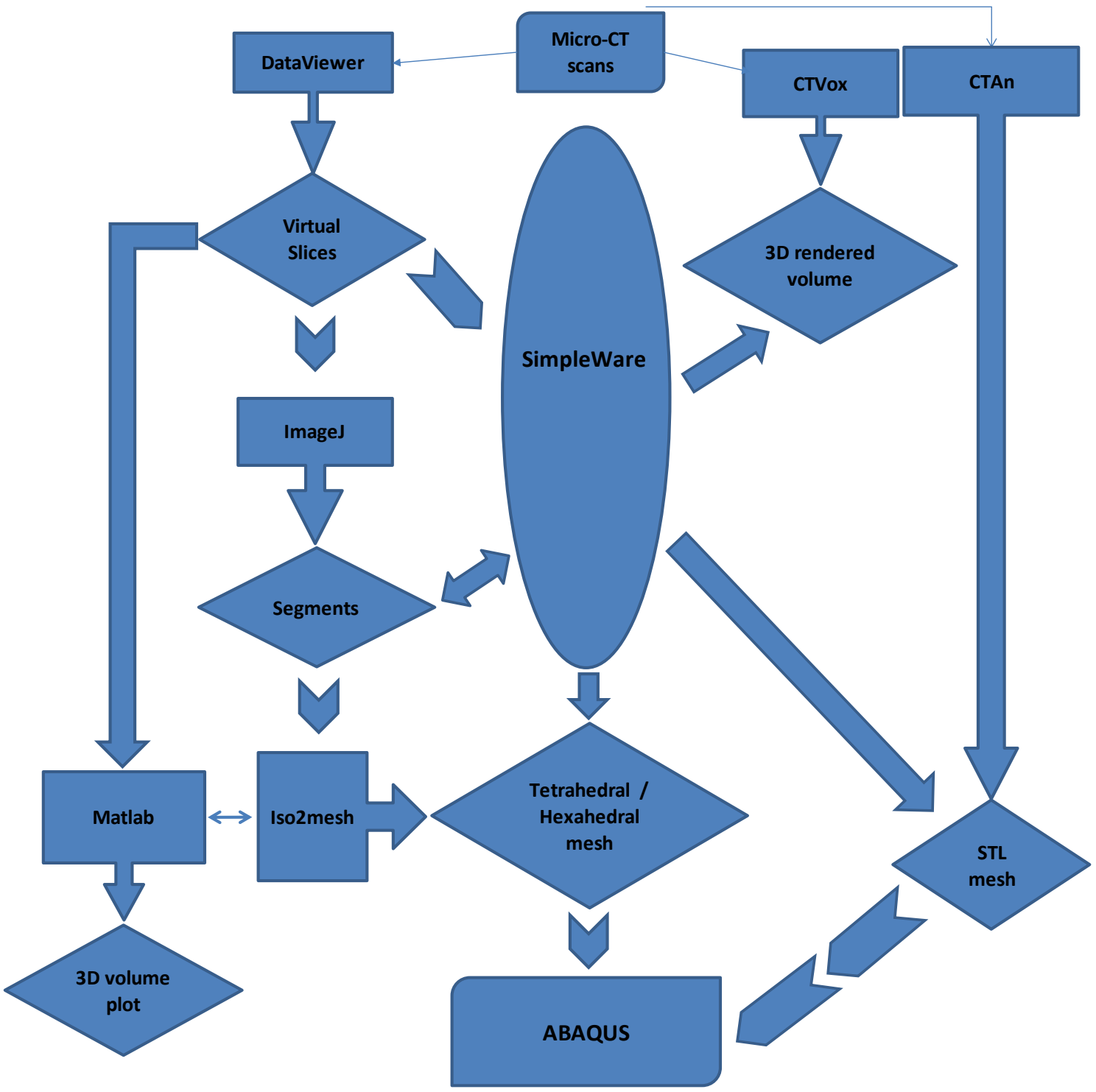

Figure 3.24: Flowchart demonstrating proposed tools for exporting microCT scans to Abaqus and their employment modes 


\section{Chapter 4:}

\section{Quantitative Analysis of Multimode Damage}

\subsection{Introduction}

Further to qualitative analysis of damage propagation described in Chapter 3, the objective of the work described in this chapter is to carry out a phenomenological study on notched laminated CFRP composites featuring orthogonal structure and subjected to constant amplitude cyclic fatigue loading. A quantitative analysis of image sequences of virtual cross-sections throughout the 3 orthogonal planes of a single sample was performed at various intervals. Hence geometry and locations of propagated cracks relative to the life cycle were statistically analyzed to obtain the damage growth trend.

Analysis resulted in defining fatigue crack growth rates, $d a / d n$ for each plane, which was interpreted in terms of the 3 damage modes; opening (mode I), in-plane shear (mode II) and out-of-plane shear (mode III). By applying LEFM laws, strain energy release rates were calculated. Considering a bi-linear triangular traction-separation curve, maximum 
tractions and separations were calculated for each of the 3 damage modes, while differentiating between modes II and III in a novel manner. Findings described in this chapter were published in a peer-reviewed journal paper [17].

\subsection{Review of Literature}

Studying the mechanical performance of PMCs can be conducted by considering RVE or repeat unit cell RUC of the structure relative to dimensional scale. Differentiating between both terms, RVE characterizes an element of the heterogeneous materials that show a statistically homogeneous behavior at the macroscopic scale, while RUC characterizes the microstructure of periodic heterogeneous materials [107]. Hence, analyzing the composite strength can be performed at different levels [108]; laminate level, ply level, or constituent level. Ply-by-ply failure is a commonly used approach to predict the failure of laminates.

\subsubsection{Failure Mechanisms}

In unidirectional plies, strain compatibility of both fibres and matrix is assumed in the case of applied axial tensile loads parallel to the fibre direction; Figure 4.1 (a), so failure occurs at the maximum strain of the fibres which is often less than that of the matrix. However, high variability in the strength of fibres results in random fibre breakage and stress concentrations at the fibre/matrix interface, which require strong interfacial bonds to prevent interfacial debonding failure. Stress concentration may be also caused by sharp variations in the geometry of part structure; e.g. at a notch or hole. In cases of transverse tensile (or compression); Figure 4.1 (b), and axial shear stresses, the failure is matrixdominated and is dependent on the local geometrical parameters [108]. 

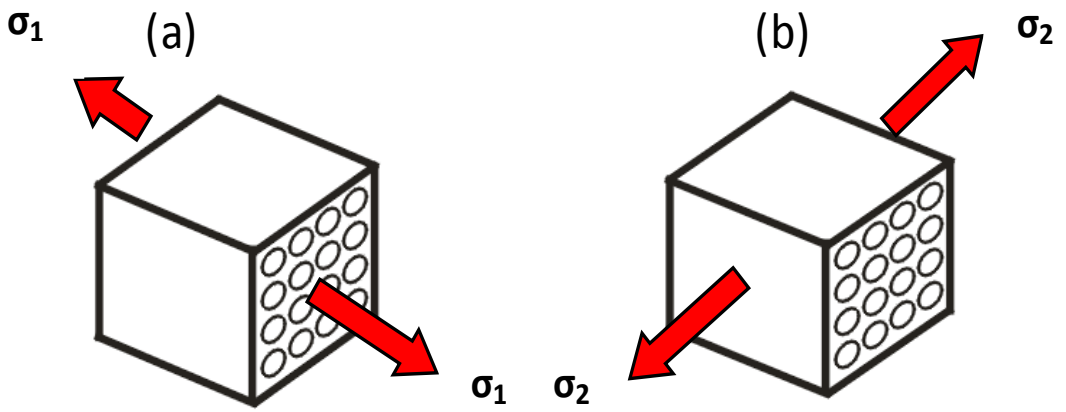

Figure 4.1: RVE of a composite subjected to (a) axial tensile load and (b) transverse tensile load

Hence, damage is an irreversible accumulated strain which is dependent on the direction of applied load(s) with respect to the orientation of fibres. Essentially failure in laminated CFRP composites is due to interfacial debonding (a matrix dominated mechanism) in combination with other mechanisms e.g. interfacial sliding. Damage may also be experienced in the form of fibre rupture (in tension), or buckling (in compression), delamination and void growth.

These mechanisms are generally attributed to the presence of internal defects, degree of strength of adhesion force between the fibres and matrix and properties of fibres relative to the matrix [7]. It is commonly observed that delamination is the prevalent type of damage often experienced in laminated CFRP composites.

Published test results for propagation of an artificially embedded delamination crack, provide data for Paris-type damage characterization caused by static loading under mode I, investigated using the Double Cantilever Beam (DCB) test [109], mode II studied by the End Notch Flexure (ENF) test [110] or a mix of both modes studied by the Mixed-Mode Bending (MMB) test for static loading [111] and fatigue loading [112,113]. Mixed-Mode End-Loaded Split (MMELS) test was also used for fatigue loading [112]. 


\subsubsection{Failure Criteria}

Failure criteria in textile composites had been extensively studied by applying continuum models; of which studies gained considerable reliability [8-14]. These criteria were implemented for simulating failure scenarios using FEA software. However, they have limited capabilities for predicting microscopic evolution of arbitrary cracks nor their complicated interacting mechanisms leading to their progression as noted by Talreja and Singh [15]. Tracing the microscopic initiation of arbitrary cracks during fatigue testing of CFRP laminates and quantitatively defining their coordinates, length and area is a cumbersome and time-intensive task if carried out manually and, to the currently available knowledge, the automation of this task had not been thoroughly addressed in literature.

To overcome the difficulties in experimental data acquisition, analytical models have been developed to predict the growth of cracks and reduce the costly testing. Several formulation was used for addressing fatigue damage propagation using quasi-static laws in conjunction with a fatigue damage degradation factor; owing to the fact the no procedures were specifically designed for damage characterization caused by cyclic fatigue loading featuring non-linearity.

Within this context, Harper and Hallet [114] developed a fatigue law in which the tractionseparation response of the interface cohesive element was discretized in a degrading manner allowing computation of the updated strain energy release rate corresponding to the rate of fatigue propagation.

De Moura and Gonçalves [115] used another approach by performing fatigue analysis on carbon-epoxy composite bolded joints by adopting a data reduction scheme that only needs 
to use the varying displacements recorded during fracture testing to estimate an equivalent crack length and consequently the strain energy release rate. As a general practice, an initial crack length should be defined.

\subsubsection{Linear Elastic Fracture Mechanics}

Propagation of existing cracks in isotropic materials subjected to fatigue loading has been extensively studied using LEFM and the well-known Paris law [116, 117] in which crack propagation rate is directly proportional to strain energy release rate in a linear relationship. Published expressions of Paris law for composites which account for contribution of both fracture modes I (opening) and II (in-plane shear) were summarized by Blanco et al. [112]. The contribution of mode III (out-of-plane shear) was found to be negligible and was disregarded in most studies [113, 114 and 106], Figure 4.2.

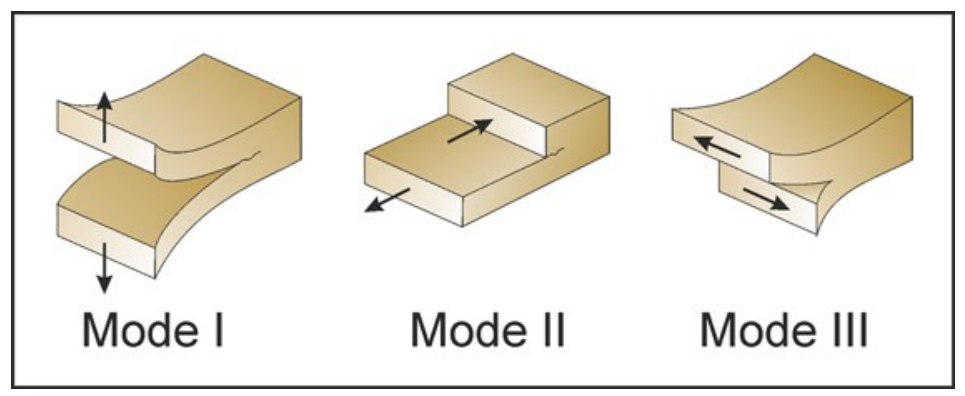

Figure 4.2: Damage modes; opening, in-plane shear and out-of-plane shear

Another approach, the VCCT was introduced by Singh and Talreja [118]. VCCT is an energy-based model in which it is assumed that the work done to open new cracks between the previously existing cracks is critical to initiate cracks in other plies by crack sliding displacement. However, the assumption of symmetrical crack propagation associated with this technique limited its popularity in addition to the commonly encountered condition in both LEFM and VCCT laws of defining a pre-existing crack length. 


\subsection{Processing of MicroCT Images}

The objective of the work described in this chapter was to perform a phenomenological study on CFRP notched sample subjected to constant amplitude cyclic fatigue loading. Sample specifications were detailed in Chapter 2. Visual images were provided using the microCT Skyscan 1173 to monitor the growth behaviour of physical cracks at prescribed intervals. These data were used to provide quantitative information on geometry and locations of propagated cracks relative to the life cycle which were then used to obtain the damage growth trend. A procedure for employing the available software tools for simulating microCT scans into Abaqus was implemented in Chapter 3.

Experimental results available in literature [110-113] provide analysis of crack propagation that pertains to artificially embedded cracks. Conversely, in this work, it was possible, by the aid of high-resolution SkyScan 1173 micro-CT, to detect pre-existing manufacturing defects in otherwise pristine samples, as shown in Figure 4.3.

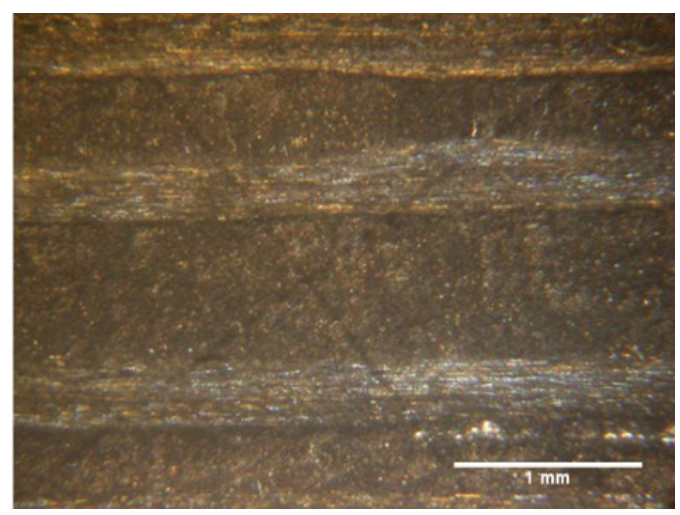

Figure 4.3: Ply layer thickness under microscope

Then, progressive non-destructive microscopic probing of damage throughout the thickness of one sample at 7 intervals during fatigue test were performed hence verifying the experimental data and providing a more realistic scenario of damage progression. Acquired raw microCT scans, which consist of approximately 1900 radiographic images 
for each phase of fatigue life ( 13000 in total), were reconstructed using NRecon software resulting in $2 \mathrm{D}$ images of area $2240 \times 2240$ pixels then processed using CTvox software for building 3D reconstructions of typically $1024 \times 1024 \times 1024$ voxels. Qualitative assessment was obtained by adjusting opacity and light levels for rendered 3D volumes, Figure 4.4, zooming on a transparent VOI on the right of the figure.

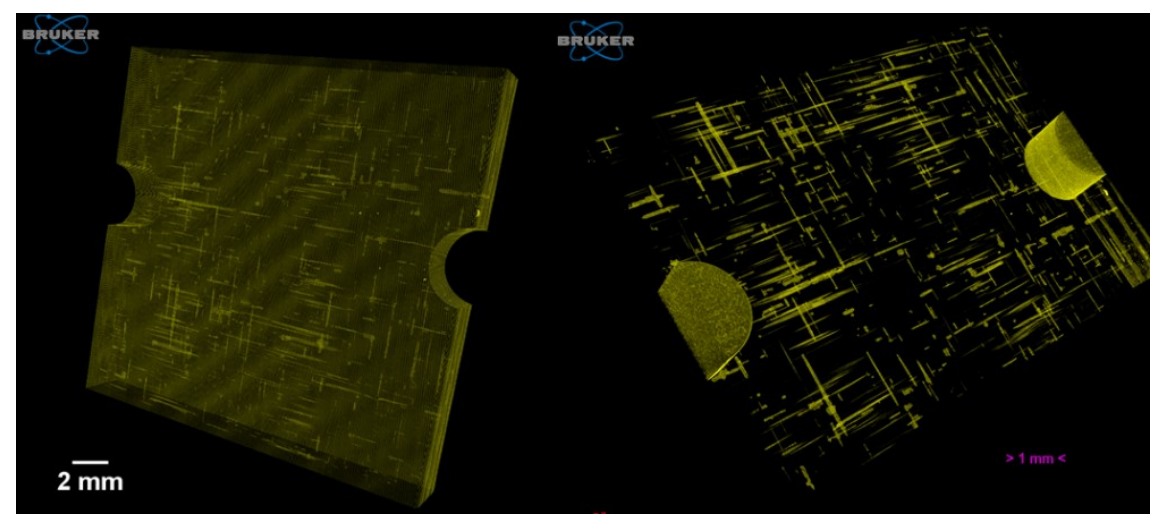

Figure 4.4: Rendered volume of cracks

Using DataViewer software, image sets for each of the 7 fatigue life phases were sliced over the 3 orthogonal 2D planes at 1 pixel intervals to provide sequences of cross-sections throughout the thickness. A VOI was defined for the area of highest stress concentration near the notches, seen in Figure 4.4, which identifies the 3 orthogonal planes. The VOI featured stacked images each of $1972 \times 1370$ pixels $(23.82 \mathrm{~mm} \times 16.55 \mathrm{~mm})$ in the $\mathrm{X}-\mathrm{Z}$ (coronal) plane, $224 \times 1370$ pixels $(2.71 \mathrm{~mm} \times 16.55 \mathrm{~mm})$ in the $Y-Z$ (sagittal) plane and $1972 \times 224$ pixels $(23.82 \mathrm{~mm} \times 2.71 \mathrm{~mm})$ in the X-Y (transverse) plane. Hence, counts of cross-sections summed up 224 images of the X-Z plane, 1972 images of the Y-Z plane and 1370 images of the X-Y plane, Figure 4.5, for each of the 7 phases.

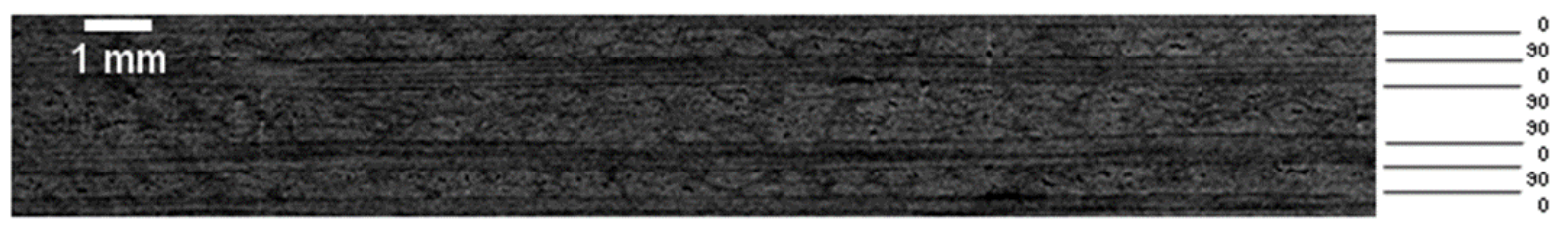

Figure 4.5: Virtual cross-section of the sample in X-Y (transverse) plane clearly identifying the 0/90 layers 


\subsection{Segmentation of Voids}

Segmentation is the process of applying a threshold to separate regions of different density levels or X-ray attenuation, in other words separate voids developed by damage from original pre-existing voids, where the detected voids correspond to areas with little or no X-ray attenuation; i.e. black areas on the 2D image.

ImageJ open-source software [102] was used to apply thresholds to the image sequences of cross-sections, where two threshold methods; minimum [119] and triangle [120] methods were visually selected and compared. The auto-threshold minimum method assumes a bimodal histogram which is iteratively smoothed using a running average of size 3 , until there are only two local maxima. Considering pixels at coordinates $(x, y)$, the threshold, $\boldsymbol{t}$, is such that [119],

$$
\boldsymbol{y}_{\boldsymbol{t}-\boldsymbol{1}}>\boldsymbol{y}_{\boldsymbol{t}}<=\boldsymbol{y}_{\boldsymbol{t}+\boldsymbol{1}}, \quad \text { Equation } 4.1
$$

Whereas the triangle algorithm assumes a maximum peak (mode) near one end of the histogram and searches towards the other end. The triangle auto threshold method was extended to find on which side of the maximum peak the data goes the furthest and searches for the threshold within that largest range.

For the $\mathrm{X}-\mathrm{Z}$ plane image sequences, the triangle auto threshold method resulted in noisy images, compared to more defined voids traced by applying the minimum auto threshold method, Figure4.6. Given that the void areas in this plane are relatively large and their values were not considerably influenced by the threshold method, the latter method was selected for separating voids for the X-Z plane. Trends of the threshold levels throughout the 224 images at fatigue life intervals were not significantly different, Figure 4.7. 


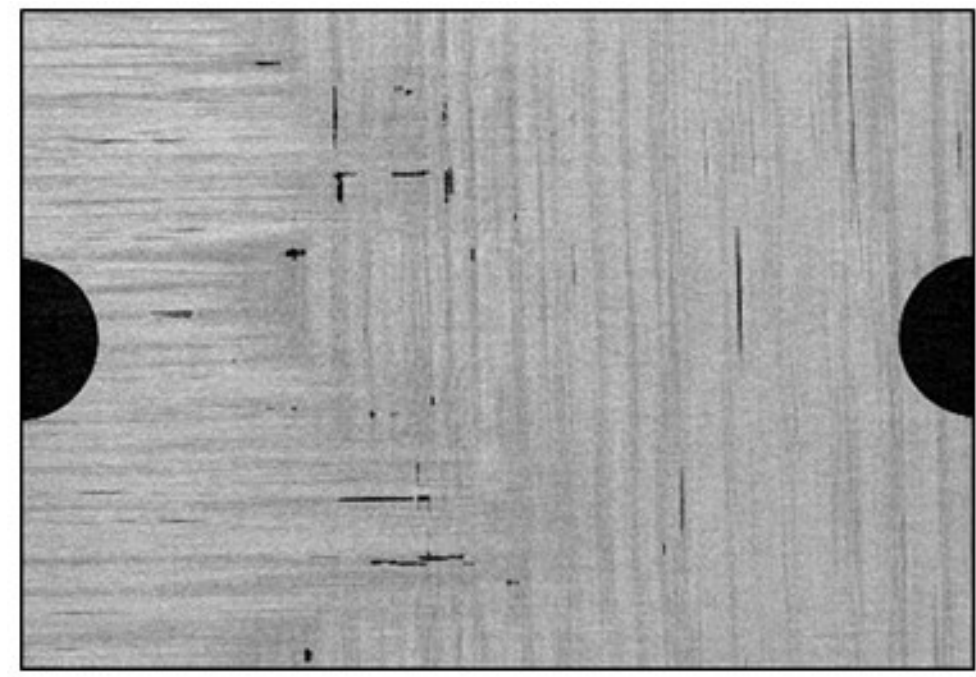

(a)

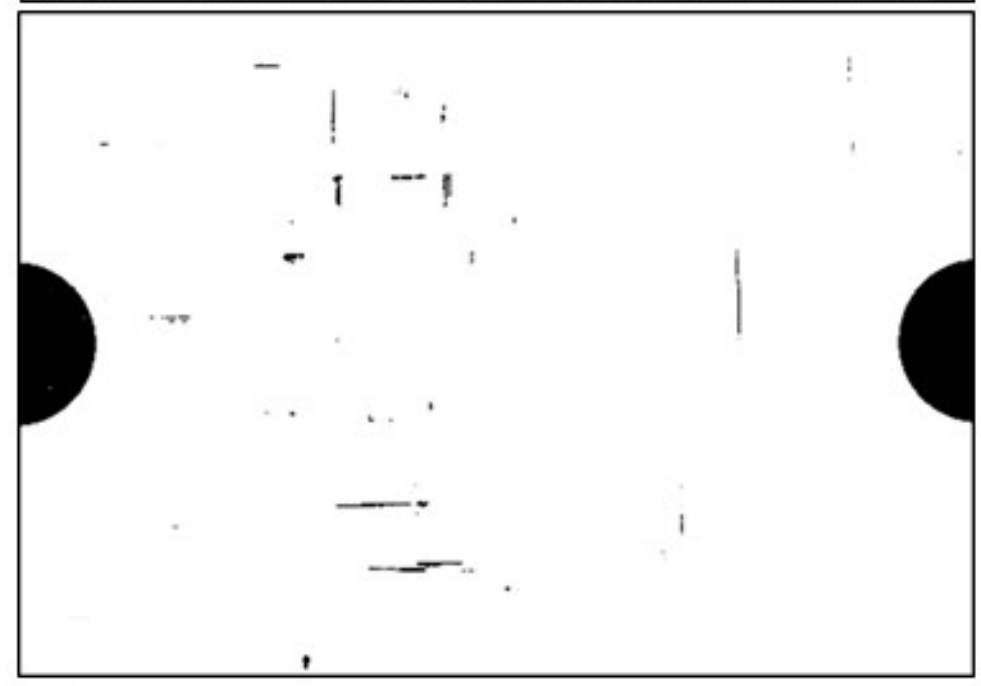

(b)

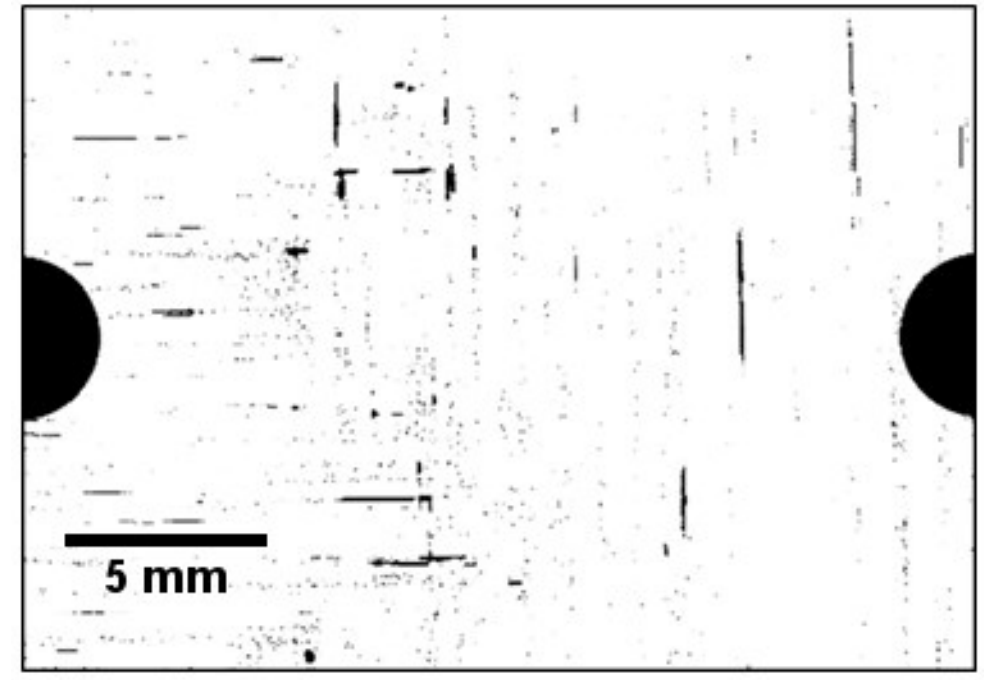

(c)

Figure 4.6: Virtual cross-section image of X-Z plane (a), minimum threshold method (b) and triangle threshold method (c) 


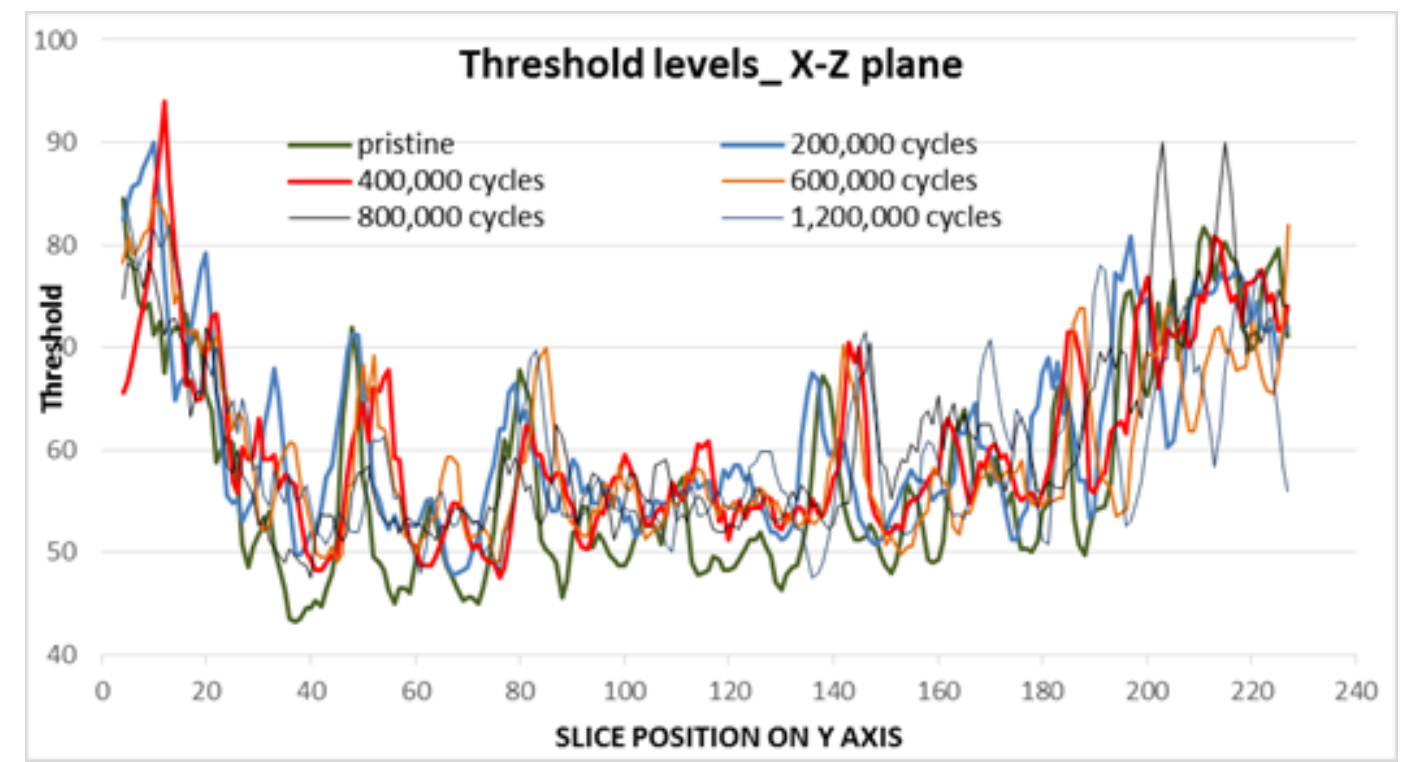

Figure 4.7: Minimum auto threshold levels for stages of fatigue life in the X-Z plane

On the contrary, for both the $\mathrm{X}-\mathrm{Z}$ and the $\mathrm{Y}-\mathrm{Z}$ planes, characterized by smaller flaws, minimum auto threshold method resulted in segmented voids of areas less than their actual values, raising concerns of resulting in misleading data of crack propagation; whereas triangle auto threshold method resulted in more realistic representation of segmented voids, Figures 4.8 and 4.9. Hence, the triangle method was selected for segmentation of these 2 planes.

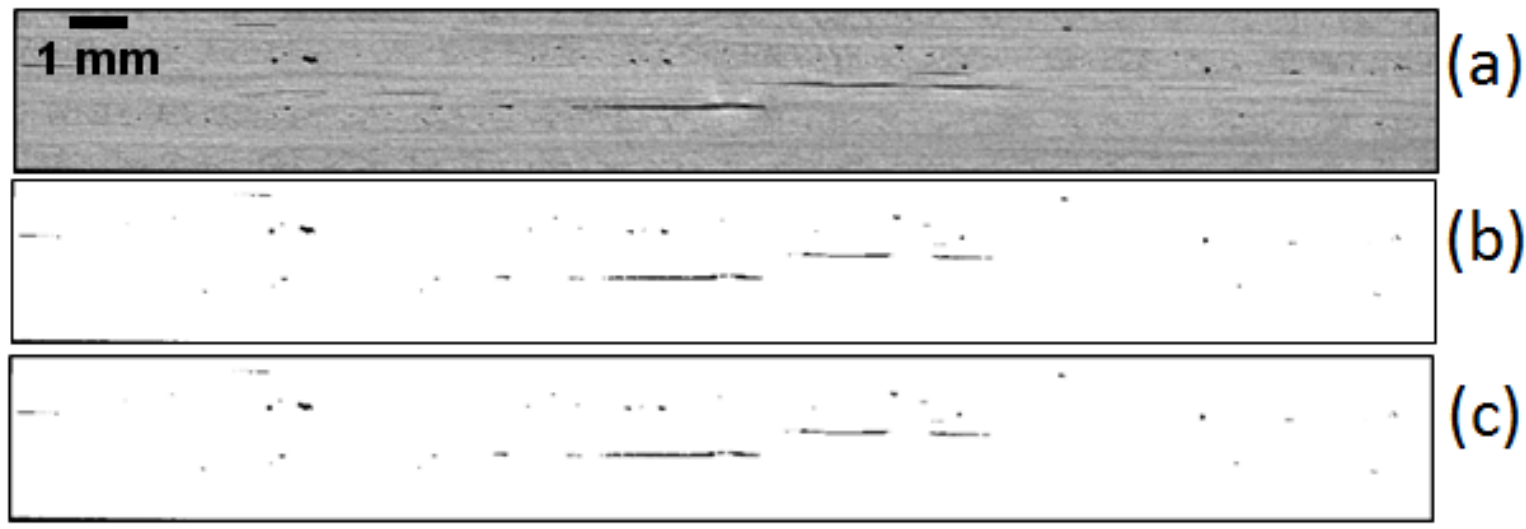

Figure 4.8: Virtual cross-section of $X$-Y plane (a), minimum threshold method (b) and triangle threshold method (c) 


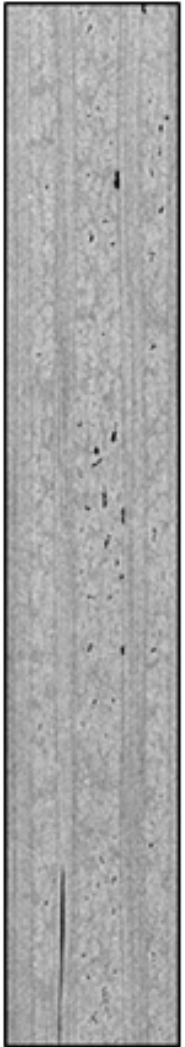

(a)

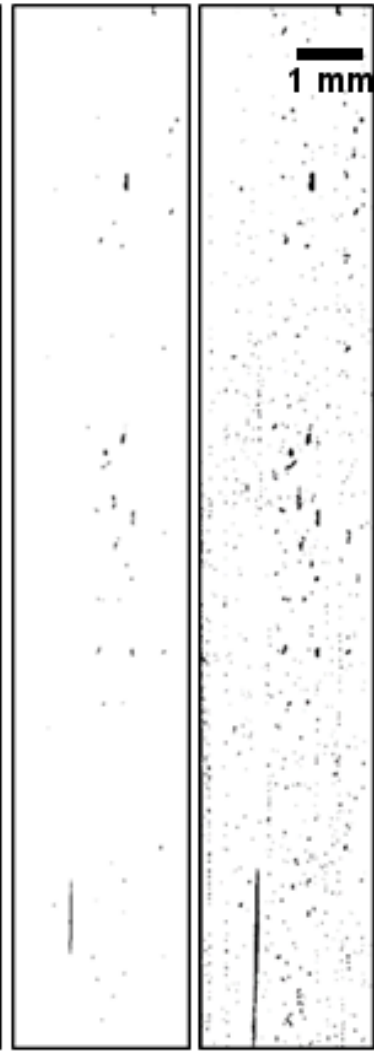

(b) (c)

Figure 4.9: Virtual cross-section of Y-Z plane (a), minimum threshold method (b) and triangle threshold method (c)

The applied threshold levels were recorded for both methods throughout the 1972 images constituting the thickness of the $\mathrm{Y}-\mathrm{Z}$ plane, and the 1370 images across the $\mathrm{X}-\mathrm{Y}$ thickness for all stages of fatigue life. Void areas were calculated and compared for further justifying the choice of triangle method. In the Y-Z plane, the auto minimum threshold levels averaged 60-70 for the images showing notches and 35-45 for the solid images, compared to 130 threshold level for solid images and 30 for the notched ones in case of the auto triangle method.

A similar pattern was observed in the $\mathrm{X}-\mathrm{Y}$ plane. No significant difference was observed in comparing phases of fatigue life. A plot of threshold levels throughout the thickness in each of these 2 spatial planes appear in Figure 4.10 for all life cycle intervals. 


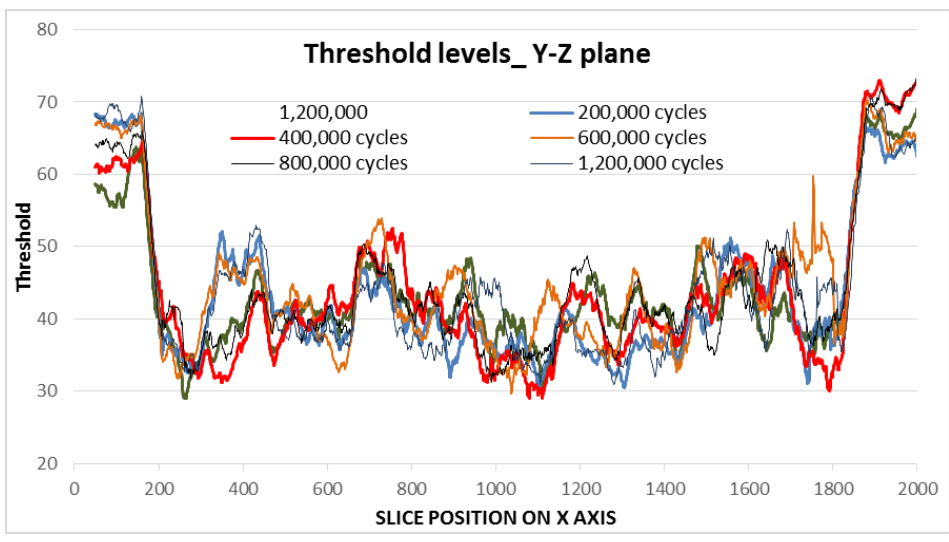

(a)

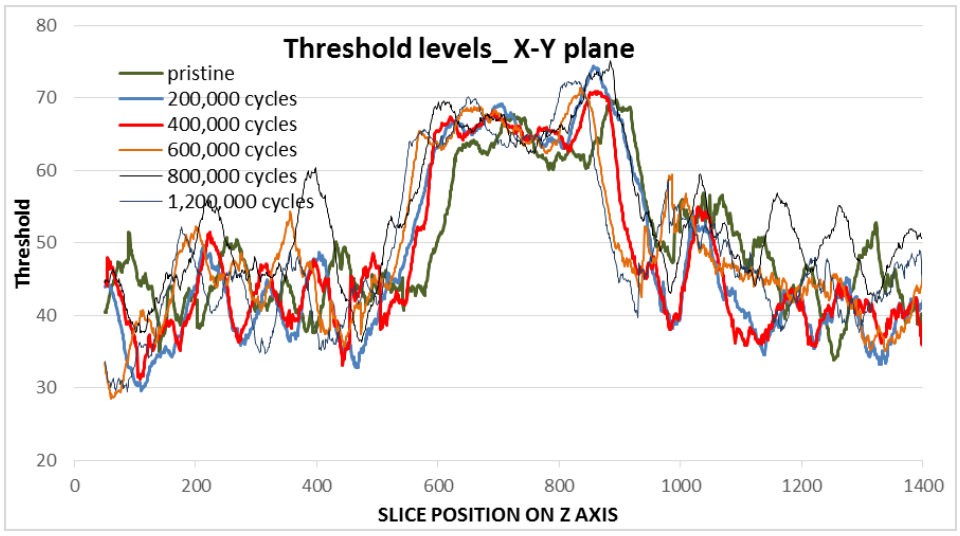

(b)

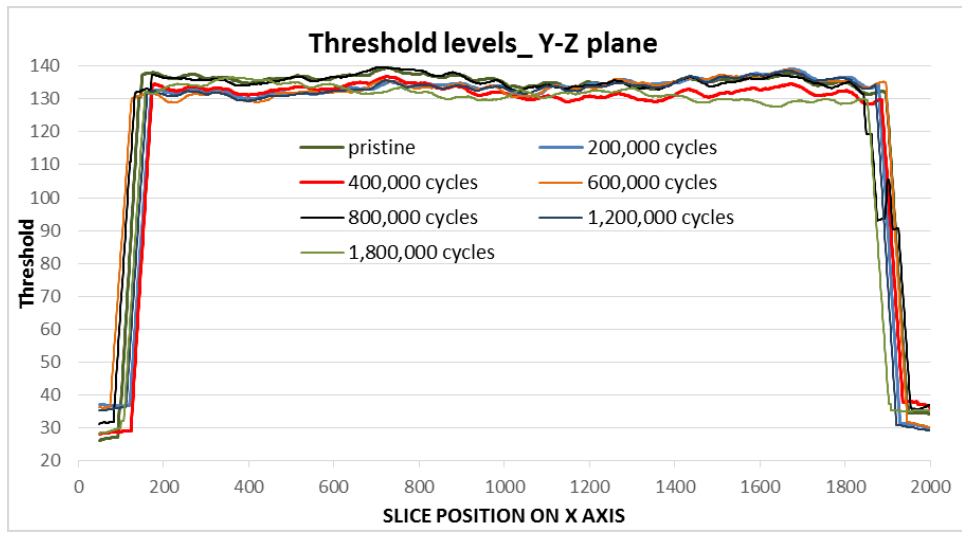

(c)

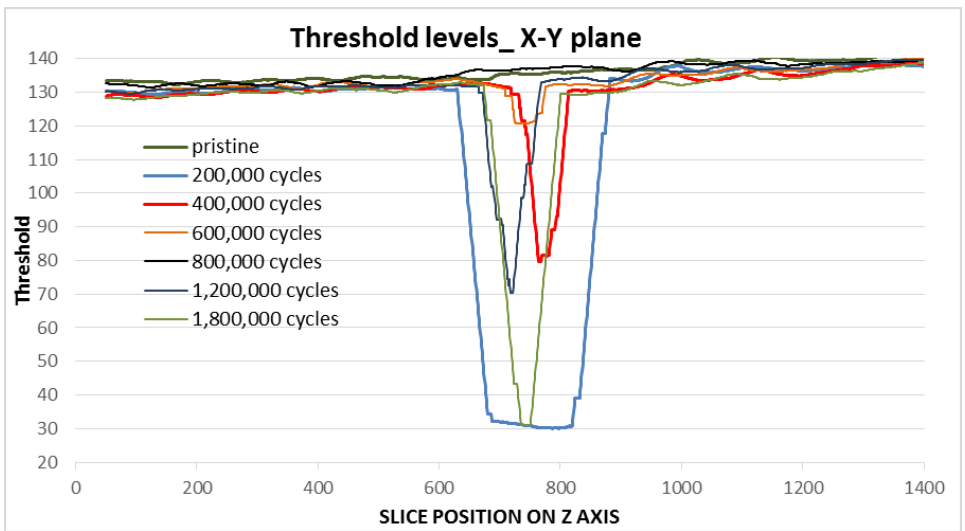

(d)

Figure 4.10: Threshold levels applied by auto 'minimum' method for the (a) $Y$-Z and the (b) $X$-Y planes and auto 'triangle' method for the (c) $Y$ - $Z$ and the (d) $X$-Y planes 


\subsection{Voids Analysis}

A higher number of voids with larger sizes and circularity was observed in the X-Z plane as compared to fewer and more longitudinal voids in both the $\mathrm{Y}-\mathrm{Z}$ and the $\mathrm{X}-\mathrm{Y}$ planes. Considering the lay-up direction of laminated plies, the voids in the $\mathrm{X}-\mathrm{Z}$ plane represent the opening (mode I) damage, where changes in size of voids correspond to fibres separation, i.e. breakage and/or splitting, Figure 4.11.

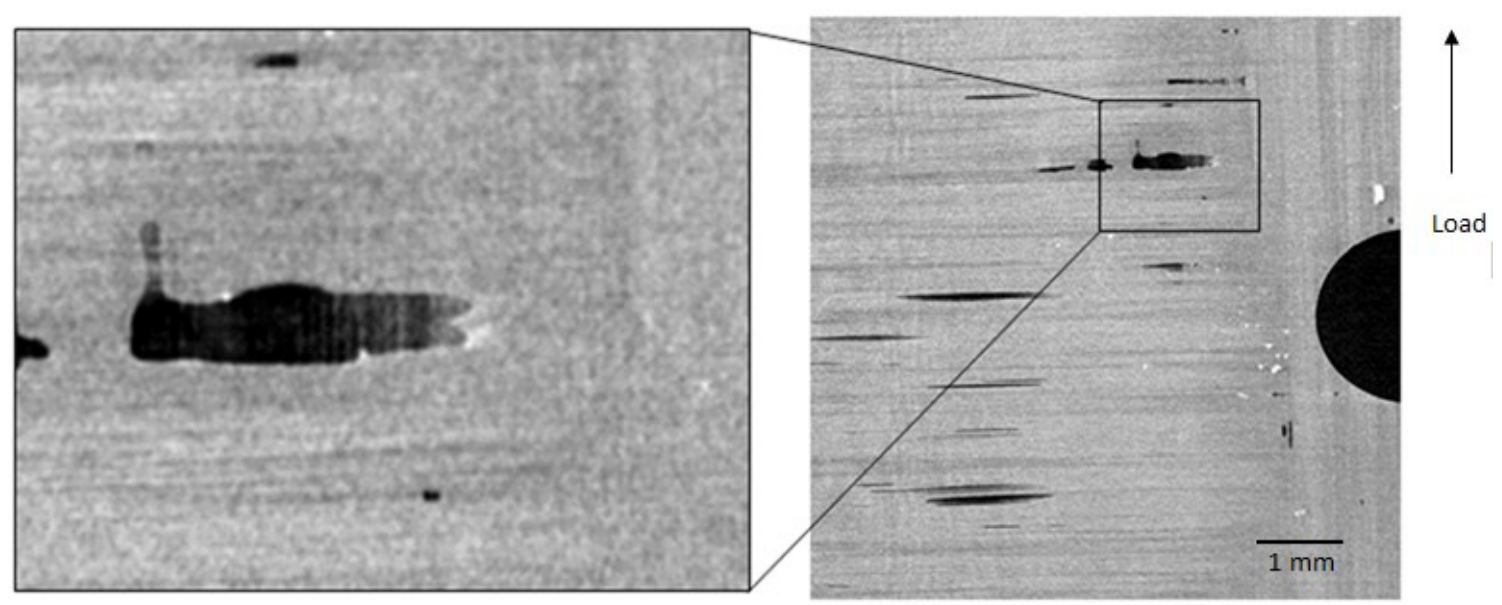

Figure 4.11: Suspected fibre breakage detected in virtual $X$-Z cross-section

On the other hand, changes in length of the longitudinal voids in the Y-Z (sagittal) plane indicate in-plane shear (mode II) damage, where parallel laminates slide in opposite directions leading to increase in length of the pre-existing voids resulting in delamination. The same delamination defects, were traced in the orthogonal X-Y (transverse) plane and the changes in length of cracks were assumed to correspond to out-of-plane shear (mode III) damage, Figure 4.2. In mode III damage, the pre-existing inter-ply cracks experience shear loads in opposite directions that change their lengths. Hence, the recorded change in crack lengths in the X-Y plane was generally of negative magnitude due to the fact that ply layers inclinely slide at acute angles if transverselly viewed resulting in decrease in their observed lengths. 
Quantitative analysis was performed by studying the cracks of which areas exceeded 1000 $\operatorname{pixel}^{2}\left(0.14 \mu \mathrm{m}^{2}\right)$ in all planes. Circularity $\left(4 \pi^{*}\right.$ area / perimeter $\left.{ }^{\wedge}\right)$ was computed by using the ImageJ [102] resulting in values ranging from 0 to $1 ; 1$ being the most circular. All circularities (0-1) in the X-Z plane were considered for analysis, whereas only longitudinal cracks of circularity below 0.25 were considered in both the $\mathrm{Y}-\mathrm{Z}$ and the $\mathrm{X}-\mathrm{Y}$ planes. Voids not meeting these criteria were neglected. Similarly, cracks that were not consistently present for 3 or more consecutive intervals at 1 pixel interval were excluded. Crack lengths were calculated assuming cracks being rectangular in shape with average width of 10 pixels as revealed from random study of voids. Length conversions and positions of voids through the thickness of each plane were calculated based on a pixel size of $12.08 \mu \mathrm{m}$.

\subsubsection{X-Z plane}

Number of initial pre-existing cracks that were detected in the $X-Z$ plane throughout its thickness was 26; the majority of which continued to open as traced at intervals of increasing the number of fatigue cycles. The average initial crack length was $2.118 \mathrm{~mm}$ in the pristine state, eventually reaching an average of $2.1475 \mathrm{~mm}$ after being subjected to 1.8 $\mathrm{x} 10^{6}$ cycles, addressing a total change in percentage void content of $1.92 \%$. A number of 10 cracks at various locations were selected for tracing their change in length, 7 of which continued to open while 3 showed a decreasing length as fatigue constant amplitude load progressed. Details of their position, progressing lengths and displacements are listed in Table 4.1. The fatigue crack growth rate, $d a / d n$ was plotted for the opening cracks, Figure 4.12 and closing cracks, Figure 4.13, whereas the displacements relative to progressive fatigue loading was plotted in Figure 4.14 for the opening cracks and Figure 4.15 for the closing cracks. The average slope of the linear trend of $d a / d n$ was $1.994 \mathrm{E}-4 \mathrm{~mm} / \mathrm{cycle}$. 


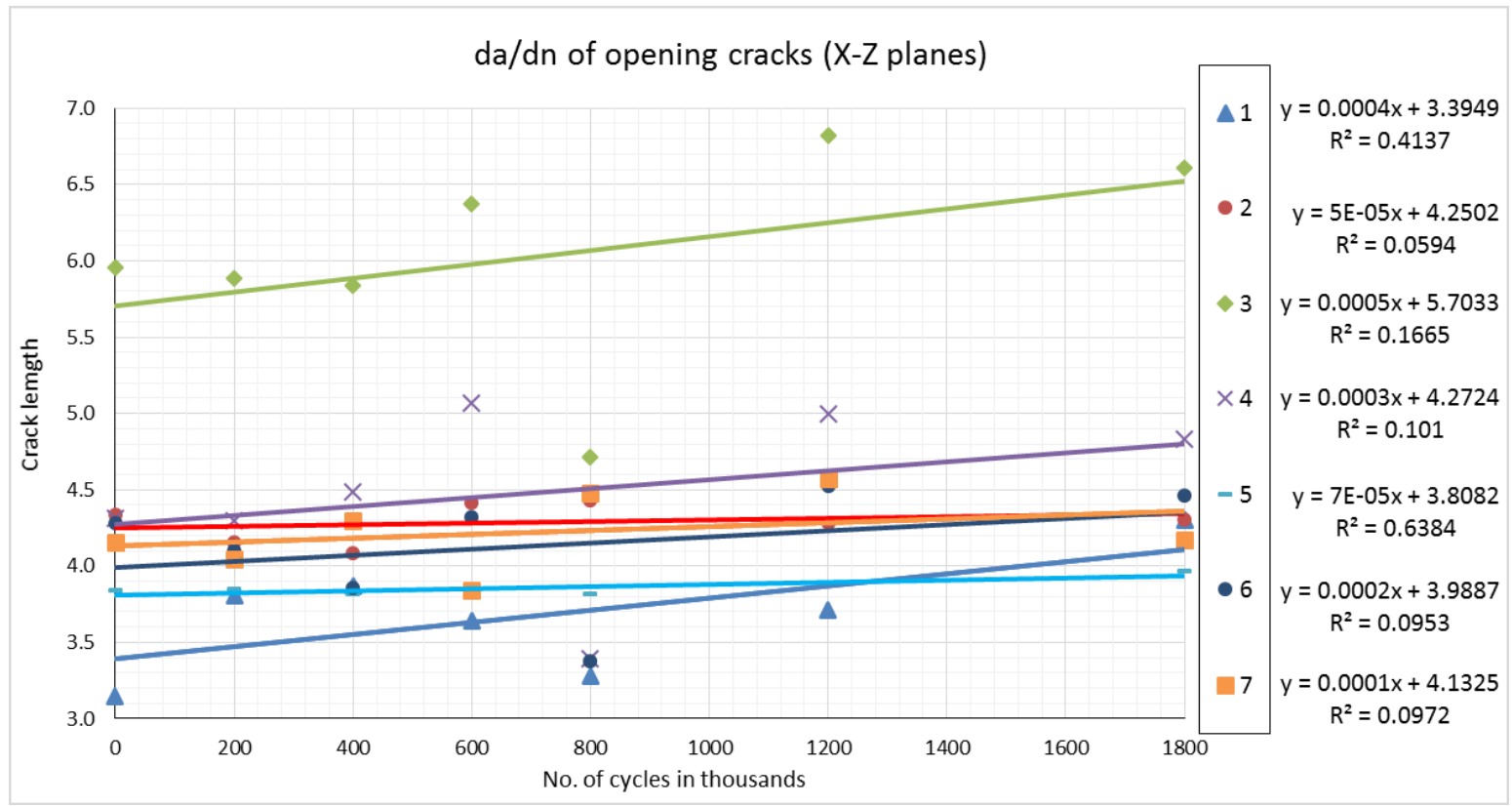

Figure 4.12: Fatigue crack growth of mode I opening cracks

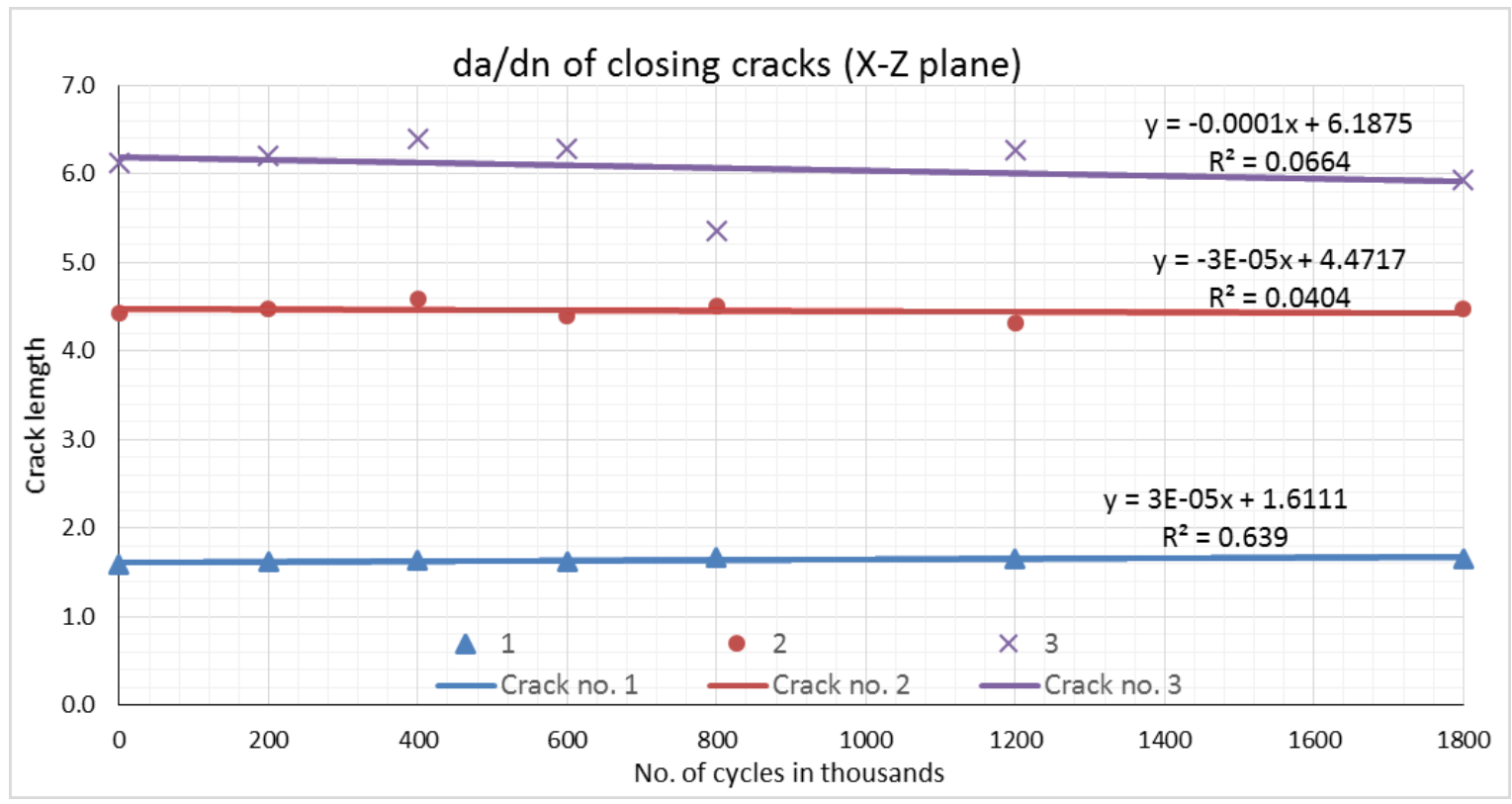

Figure 4.13: Fatigue crack growth of mode I closing cracks 


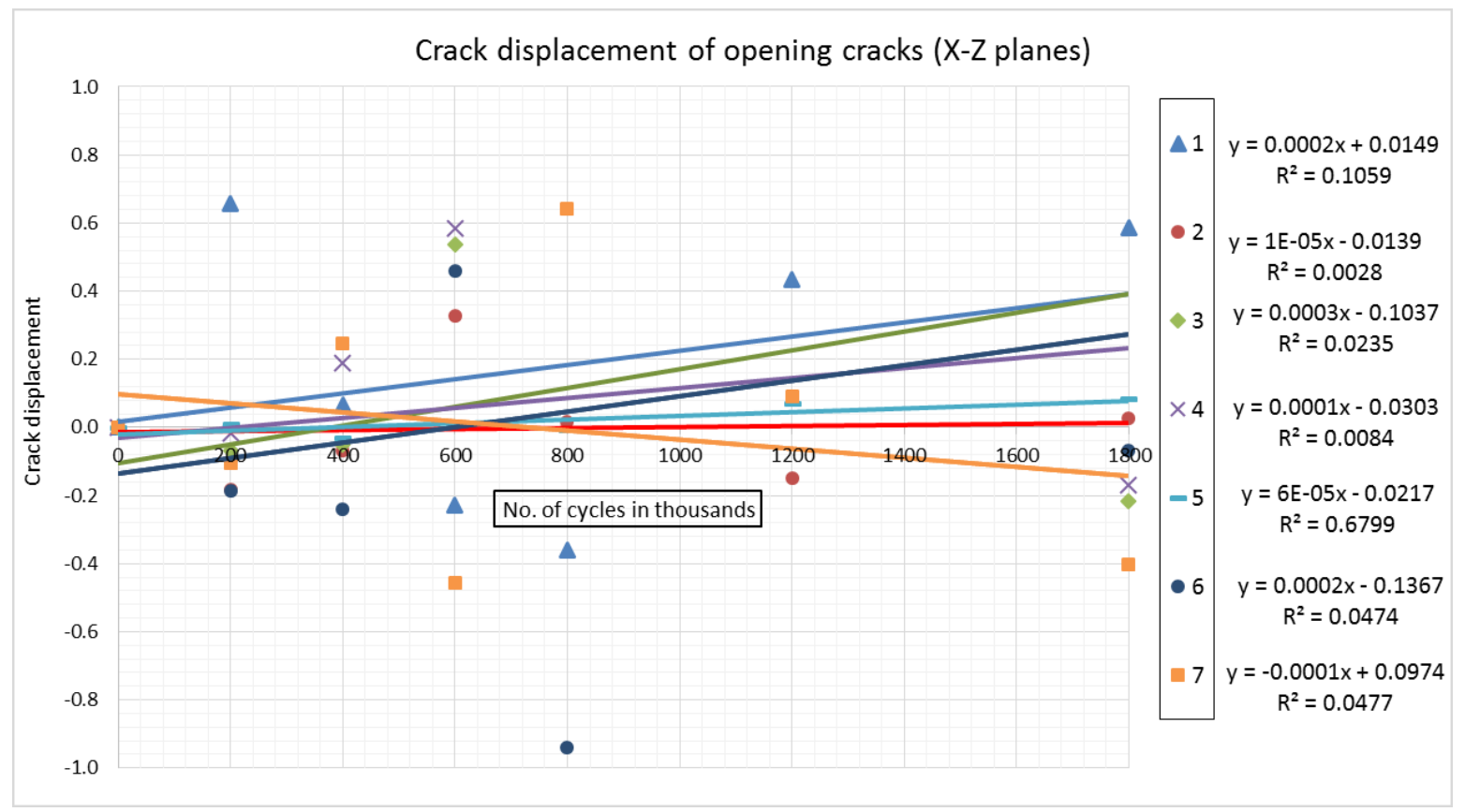

Figure 4.14: Crack displacements of mode I opening cracks

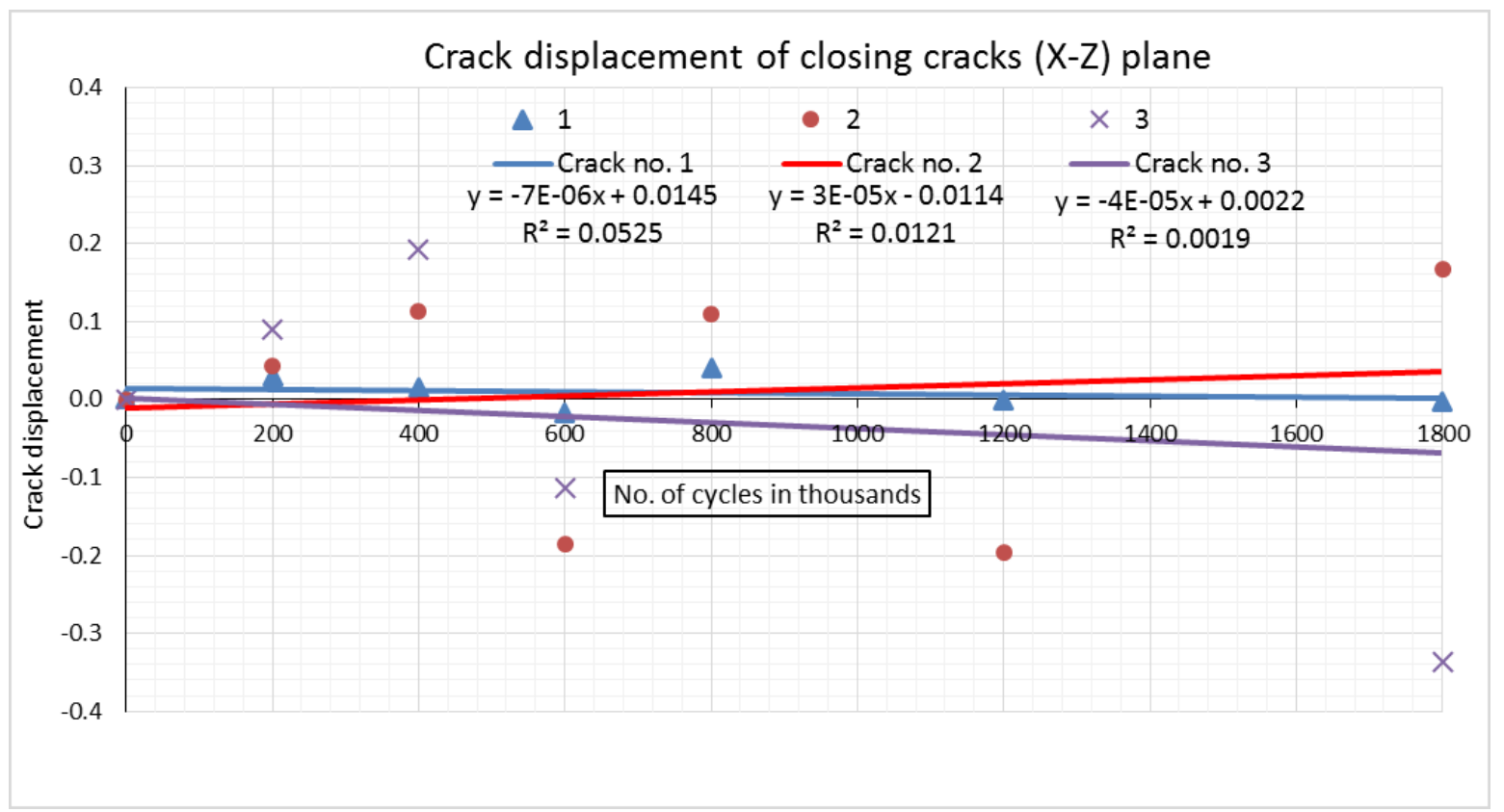

Figure 4.15: Crack displacements of mode I closing cracks 
Table 4.1: Cracks coordinates and change in length for X-Z plane (cycles in thousands)

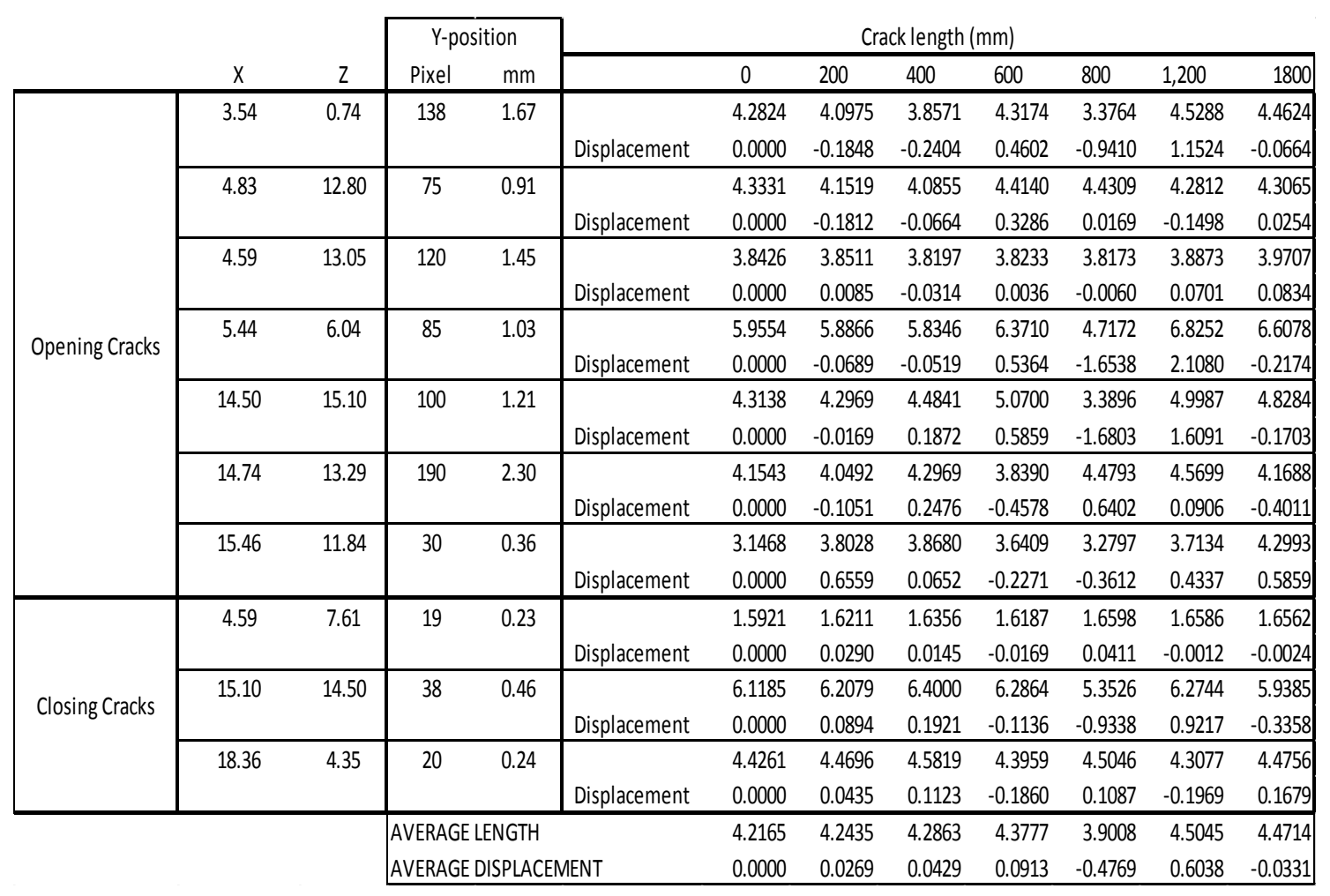

\subsubsection{Y-Z plane}

The same procedure was followed for the $\mathrm{Y}-\mathrm{Z}$ plane for delamination detection. However, a lower percentage of cracks continued to open with increasing numbers of fatigue cycles as compared to the $\mathrm{X}-\mathrm{Z}$ plane, revealed by the total change in percentage void content that was dereased by $-19.49 \%$, and the average initial crack length that was decreased from $1.7527 \mathrm{~mm}$ in the pristine state to $1.7105 \mathrm{~mm}$ after being subjected to $1.8 \times 10^{6}$ cycles. The largest 10 cracks at various locations were selected for detailed length tracking, 7 of which continued to open while 3 showed a decreasing length as fatigue load progressed. Details of their position, progressing lengths and displacements were listed in Table 4.2. The fatigue crack growth rate, $d a / d n$ was plotted for the opening cracks, Figure 4.16 and closing 
cracks, Figure 4.17, whereas the displacements relative to progressive fatigue loading was plotted in Figure 4.18 for the opening cracks and Figure4.19for the closing cracks. The average slope of the linear trend of $d a / d n$ was observed to be $9 \mathrm{E}-5 \mathrm{~mm} /$ cycle. The decrease in average crack length can be attributed to continuous stress redistribution which results in closing some cracks as others open. An overall trend of increase in average crack length in delamination is eventually expected as fatigue load progresses, agreeing with the published qualitative results proving that transverse ply cracks occur at an earlier stage of loading than that causing splits, delamination and then fibre breakage, respectively [89].

Table 4.2: Cracks coordinates and change in length for $Y$-Z plane (cycles in thousands)

\begin{tabular}{|c|c|c|c|c|c|c|c|c|c|c|c|c|}
\hline & \multirow[b]{2}{*}{ Y } & \multirow[b]{2}{*}{ Z } & \multicolumn{2}{|c|}{ X-position } & \multicolumn{8}{|c|}{ Crack length (mm) } \\
\hline & & & Pixel & $\mathrm{mm}$ & & 0 & 200 & 400 & 600 & 800 & 1,200 & 1800 \\
\hline \multirow{14}{*}{ Opening cracks } & \multirow[t]{2}{*}{0.64} & \multirow[t]{2}{*}{11.11} & \multirow[t]{2}{*}{1370} & \multirow[t]{2}{*}{16.55} & & 1.3820 & 1.3493 & 1.2660 & 1.3373 & 1.2950 & 1.4399 & 1.4448 \\
\hline & & & & & Displacement & 0.0000 & -0.0326 & -0.0834 & 0.0713 & -0.0423 & 0.1450 & 0.0048 \\
\hline & \multirow[t]{2}{*}{0.68} & \multirow[t]{2}{*}{12.44} & \multirow[t]{2}{*}{1825} & \multirow[t]{2}{*}{22.05} & & 1.8265 & 1.7444 & 1.7214 & 1.7419 & 1.8337 & 1.9098 & 1.7806 \\
\hline & & & & & Displacement & 0.0000 & -0.0821 & -0.0230 & 0.0205 & 0.0918 & 0.0761 & -0.1293 \\
\hline & \multirow[t]{2}{*}{1.03} & \multirow[t]{2}{*}{1.93} & \multirow[t]{2}{*}{300} & \multirow[t]{2}{*}{3.62} & & 2.0790 & 2.1249 & 2.3290 & 2.5730 & 2.6661 & 2.6950 & 2.8183 \\
\hline & & & & & Displacement & 0.0000 & 0.0459 & 0.2042 & 0.2440 & 0.0930 & 0.0290 & 0.1232 \\
\hline & \multirow[t]{2}{*}{1.69} & \multirow[t]{2}{*}{11.48} & \multirow[t]{2}{*}{1650} & \multirow[t]{2}{*}{19.93} & & 1.6199 & 1.5934 & 1.5450 & 1.6284 & 1.5994 & 2.1225 & 1.7431 \\
\hline & & & & & Displacement & 0.0000 & -0.0266 & -0.0483 & 0.0834 & -0.0290 & 0.5231 & -0.3793 \\
\hline & \multirow[t]{2}{*}{1.69} & \multirow[t]{2}{*}{12.68} & \multirow[t]{2}{*}{770} & \multirow[t]{2}{*}{9.30} & & 1.4883 & 1.3783 & 1.4158 & 1.4544 & 1.4810 & 1.4508 & 1.4315 \\
\hline & & & & & Displacement & 0.0000 & -0.1099 & 0.0374 & 0.0387 & 0.0266 & -0.0302 & -0.0193 \\
\hline & \multirow[t]{2}{*}{1.81} & \multirow[t]{2}{*}{6.46} & \multirow[t]{2}{*}{1775} & \multirow[t]{2}{*}{21.44} & & 2.2469 & 2.2239 & 2.3351 & 2.1889 & 1.7830 & 2.8859 & 2.1696 \\
\hline & & & & & Displacement & 0.0000 & -0.0230 & 0.1111 & -0.1462 & -0.4059 & 1.1029 & -0.7163 \\
\hline & \multirow[t]{2}{*}{1.81} & 10.27 & 1850 & 22.35 & & 2.7905 & 2.3049 & 3.0188 & 2.4619 & 2.4945 & 2.8388 & 2.9898 \\
\hline & & & & & Displacement & 0.0000 & -0.4856 & 0.7139 & -0.5569 & 0.0326 & 0.3443 & 0.1510 \\
\hline & 1.99 & 2.90 & 1320 & 15.95 & & 1.6779 & 1.7286 & 1.7093 & 1.7323 & 1.6562 & 1.6344 & 1.7033 \\
\hline & & & & & Displacement & 0.0000 & 0.0507 & -0.0193 & 0.0230 & -0.0761 & -0.0217 & 0.0689 \\
\hline Olosing crack & 1.69 & 12.08 & 350 & 4.23 & & 2.6793 & 2.4462 & 2.6455 & 2.4692 & 2.5839 & 2.5199 & 2.4981 \\
\hline Clusilitg tiacks & & & & & Displacement & 0.0000 & -0.2331 & 0.1993 & -0.1764 & 0.1148 & -0.0640 & -0.0217 \\
\hline & 2.05 & 1.45 & 475 & 5.74 & & 1.9050 & 1.8821 & 2.0306 & 1.9207 & 1.9207 & 1.8627 & 1.9062 \\
\hline & & & & & Displacement & 0.0000 & -0.0230 & 0.1486 & -0.1099 & 0.0000 & -0.0580 & 0.0435 \\
\hline & & & AVERA & ENGTH & & 1.9695 & 1.8776 & 2.0017 & 1.9508 & 1.9314 & 2.1360 & 2.0485 \\
\hline & & & AVERA & ISPLAC| & MENT & 0.0000 & -0.0919 & 0.1241 & -0.0509 & -0.0194 & 0.2046 & -0.0875 \\
\hline
\end{tabular}




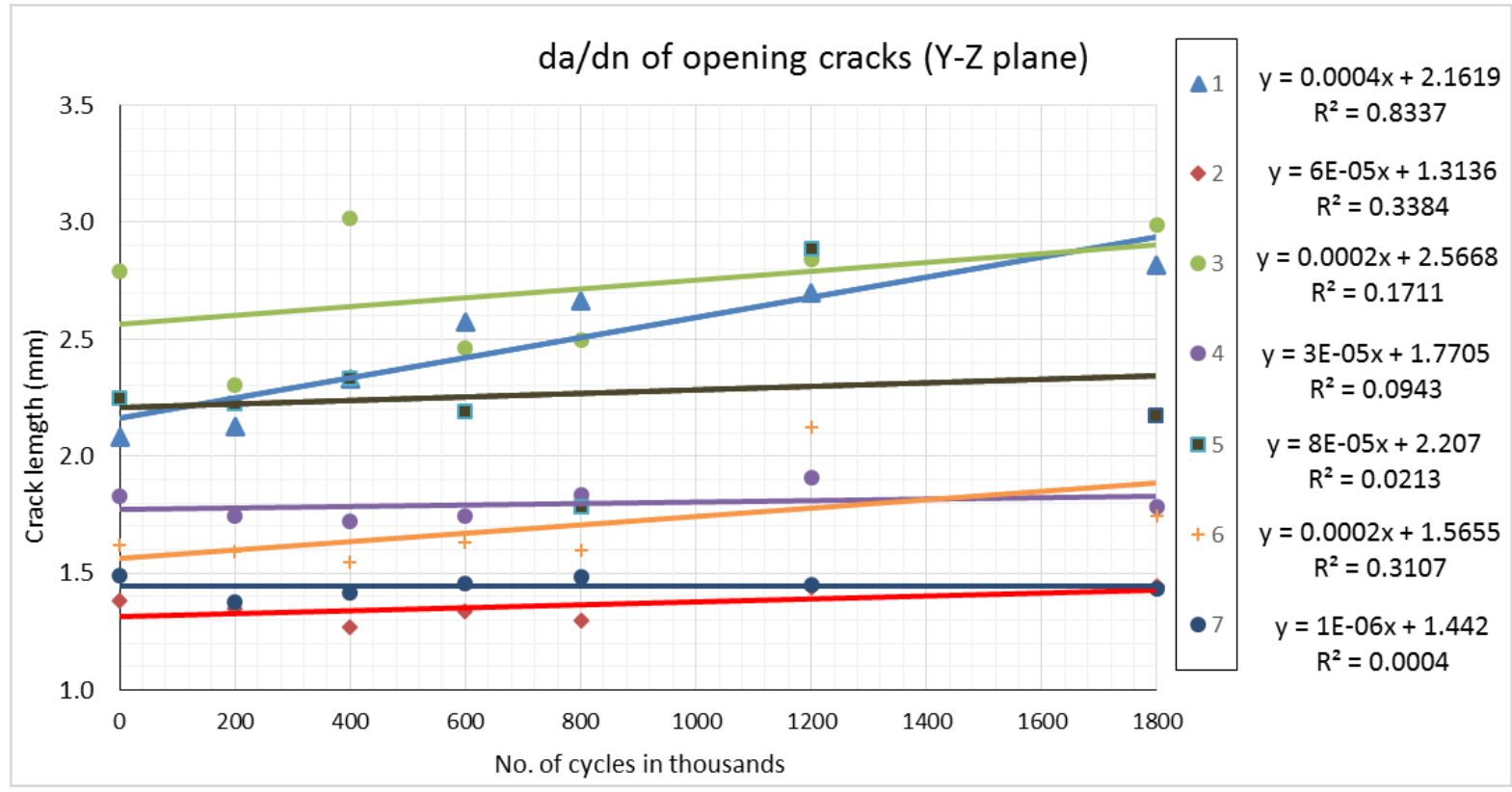

Figure 4.16: Fatigue crack growth of mode II opening cracks

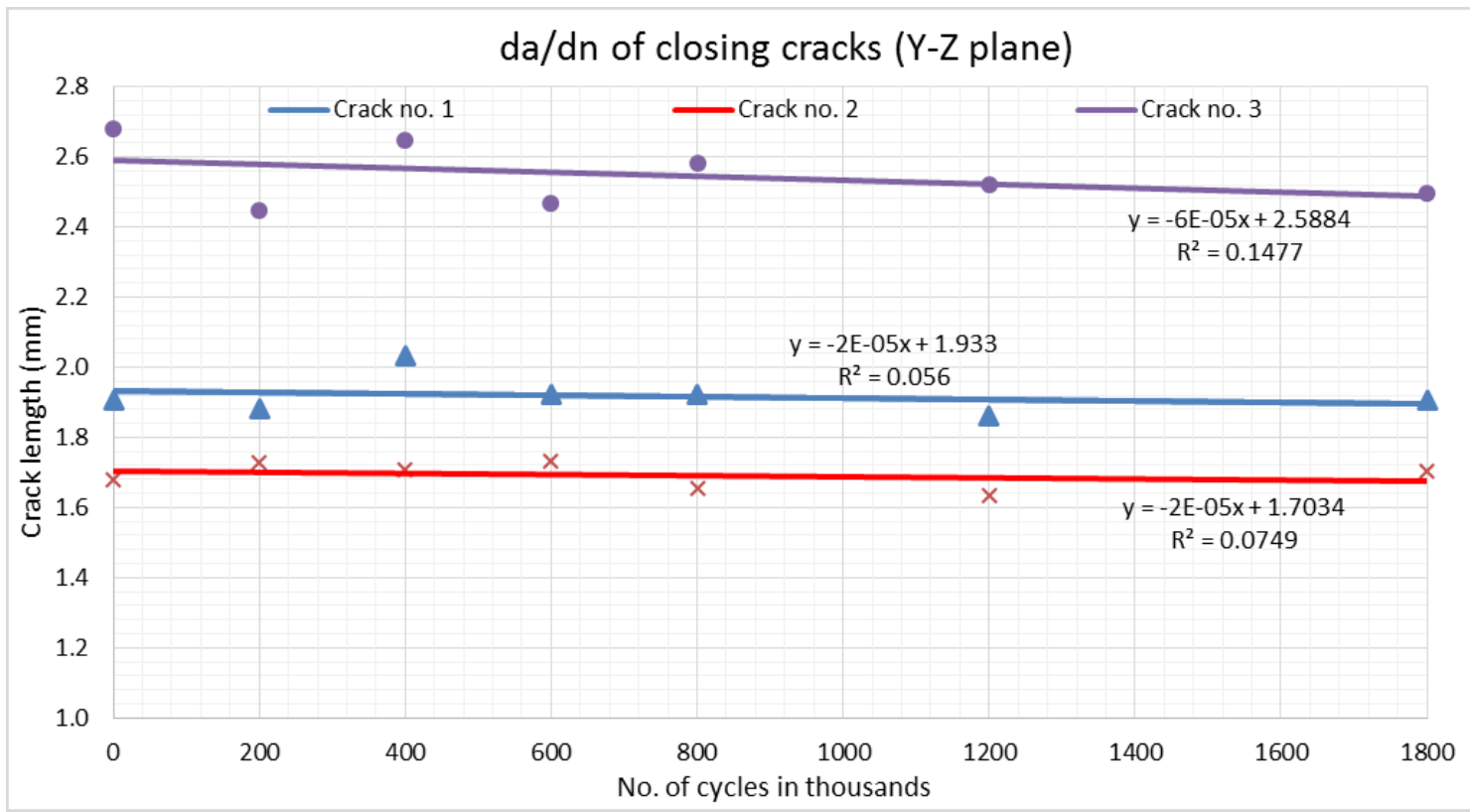

Figure 4.17: Fatigue crack growth of mode II closing cracks 


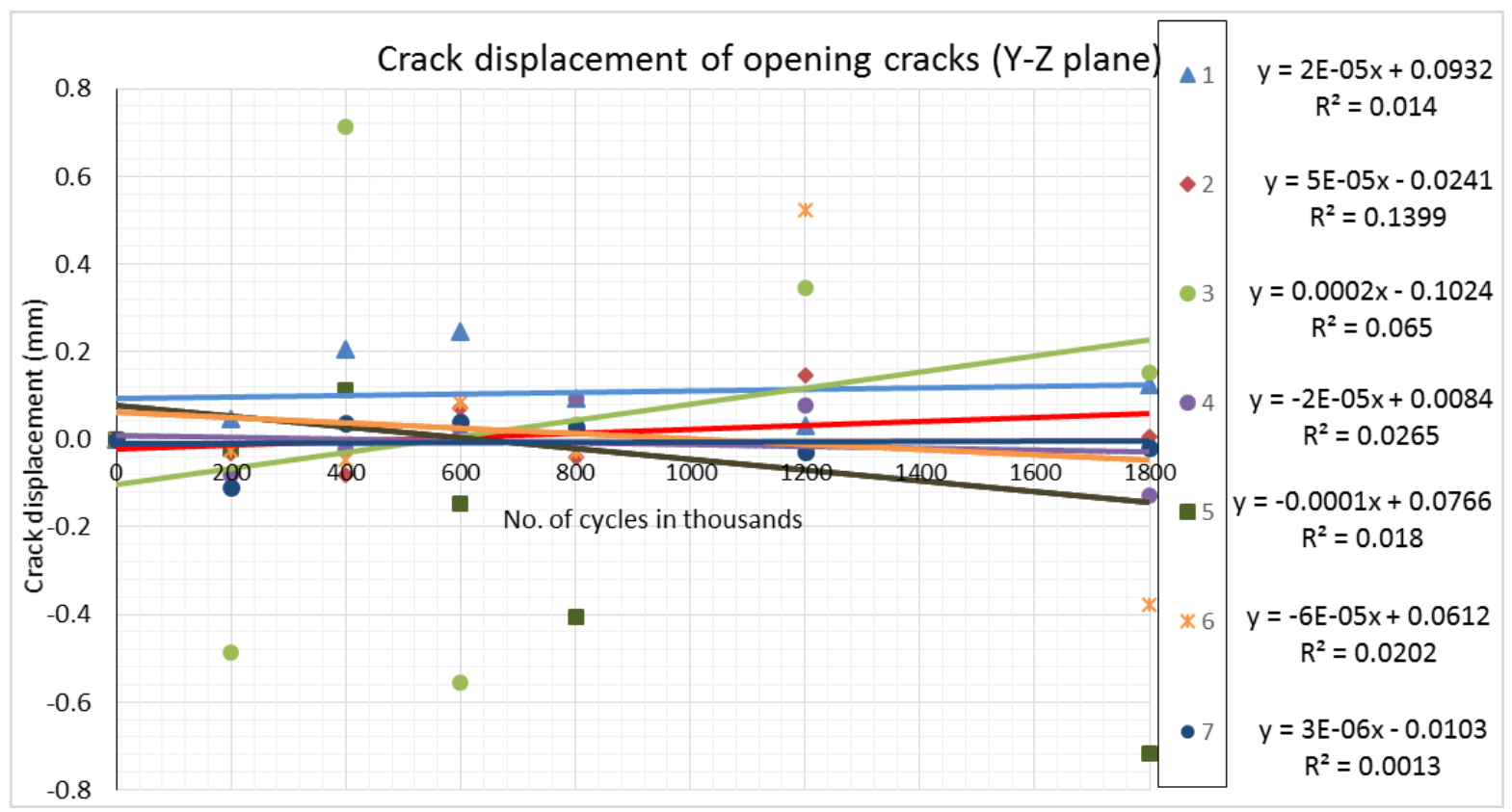

Figure 4.18: Crack displacements of mode II opening cracks

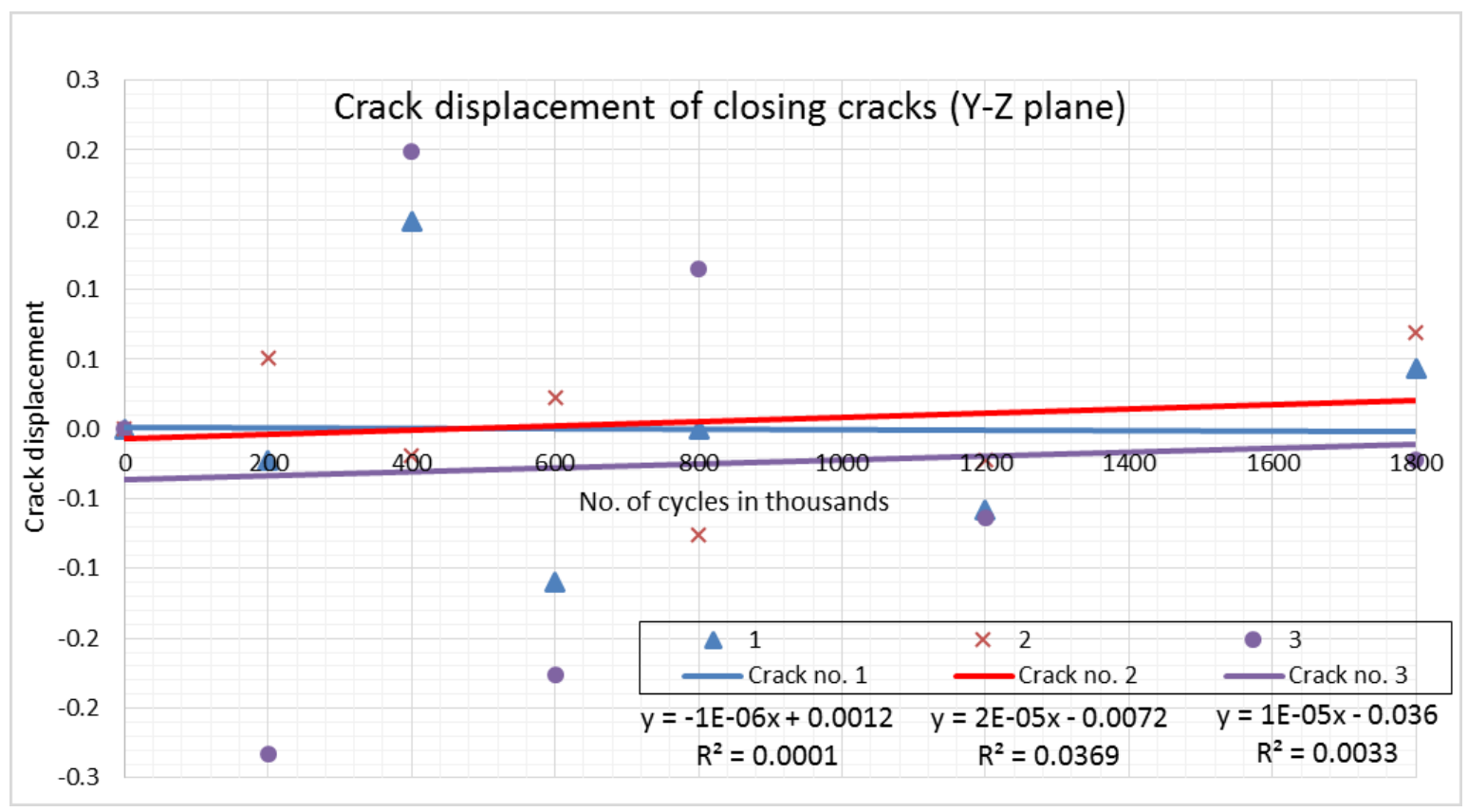

Figure 4.19: Crack displacements of mode II closing cracks 


\subsubsection{X-Y plane}

By following the same procedure for the X-Y plane, the percentage of closing cracks were observed to be higher than that of the opening ones. For that reason, out of 10 cracks that were selected for tracing their change in length, 3 continued to open while 7 decreased in length as fatigue load progressed. The total change in percentage void content that was dereased by $-11.62 \%$. The average initial crack length that was decreased from $1.8744 \mathrm{~mm}$ in the pristine state to $1.74 \mathrm{~mm}$ after being subjected to $1.8 \times 10^{6}$ cycles. Details of their position, progressing lengths and displacements were listed in Table 4.3. The fatigue crack growth rate, $d a / d n$ was plotted for the opening cracks, Figure 4.20 and closing cracks, Figure 4.21, whereas the displacements relative to progressive fatigue loading was plotted in Figure 4.22 for the opening cracks and Figure 4.23 for the closing cracks. The average slope of the linear trend of $d a / d n$ was observed as $-7 \mathrm{E}-5 \mathrm{~mm} / \mathrm{cycle}$.

Table 4.3: Cracks coordinates and change in length for X-Y plane (cycles in thousands)

\begin{tabular}{|c|c|c|c|c|c|c|c|c|c|c|c|c|}
\hline & \multirow[b]{2}{*}{$\mathrm{x}$} & \multirow[b]{2}{*}{ Y } & \multicolumn{2}{|c|}{ Z-position } & \multicolumn{8}{|c|}{ Crack length $(\mathrm{mm})$} \\
\hline & & & Pixel & $\mathrm{mm}$ & & 0 & 200 & 400 & 600 & 800 & 1,200 & 1800 \\
\hline \multirow{6}{*}{ Opening Cracks } & 4.23 & 2.17 & 1200 & 14.50 & & 3.3860 & 3.1227 & 3.3570 & 3.3884 & 3.3727 & 3.3075 & 3.3752 \\
\hline & & & & & Displacement & 0.0000 & -0.2633 & 0.2344 & 0.0314 & -0.0157 & -0.0652 & 0.0676 \\
\hline & 13.29 & 0.43 & 170 & 2.05 & & 2.7458 & 2.8195 & 2.8219 & 2.7820 & 2.8134 & 2.8183 & 2.8050 \\
\hline & & & & & Displacement & 0.0000 & 0.0737 & 0.0024 & -0.0399 & 0.0314 & 0.0048 & -0.0133 \\
\hline & 14.50 & 1.21 & 200 & 2.42 & & 2.1877 & 2.2759 & 2.2203 & 2.2928 & 2.1418 & 2.5006 & 2.3616 \\
\hline & & & & & Displacement & 0.0000 & 0.0882 & -0.0556 & 0.0725 & -0.1510 & 0.3588 & -0.1389 \\
\hline \multirow{14}{*}{ Closing Cracks } & 4.83 & 1.03 & 700 & 8.46 & & 2.7494 & 3.0152 & 2.6455 & 2.4257 & 2.4679 & 2.3314 & 1.9098 \\
\hline & & & & & Displacement & 0.0000 & 0.2658 & -0.3696 & -0.2199 & 0.0423 & -0.1365 & -0.4216 \\
\hline & 5.07 & 1.45 & 270 & 3.26 & \multirow[b]{2}{*}{ Displacement } & 2.962016 & 2.9185 & 2.9113 & 2.9125 & 2.9451 & 2.9318 & 2.8775 \\
\hline & & & & & & 0.0000 & -0.0435 & -0.0072 & 0.0012 & 0.0326 & -0.0133 & -0.0544 \\
\hline & \multirow[t]{2}{*}{5.44} & \multirow[t]{2}{*}{1.21} & 100 & 1.21 & \multirow[b]{2}{*}{ Displacement } & 2.3477 & 2.2759 & 2.2203 & 2.0693 & 2.1176 & 2.3169 & 2.1768 \\
\hline & & & & & & 0.0000 & -0.0719 & -0.0556 & -0.1510 & 0.0483 & 0.1993 & -0.1401 \\
\hline & \multirow[t]{2}{*}{7.25} & \multirow[t]{2}{*}{1.57} & 550 & 6.64 & \multirow[b]{2}{*}{ Displacement } & 2.4245 & 2.2940 & 2.4631 & 2.2771 & 2.2976 & 2.2976 & 2.2529 \\
\hline & & & & & & 0.0000 & -0.1305 & 0.1691 & -0.1860 & 0.0205 & 0.0000 & -0.0447 \\
\hline & \multirow[t]{2}{*}{11.48} & \multirow[t]{2}{*}{1.51} & 220 & 2.66 & \multirow[b]{2}{*}{ Displacement } & 1.8978 & 2.0693 & 1.9485 & 1.9473 & 1.8941 & 1.9715 & 1.8809 \\
\hline & & & & & & 0.0000 & 0.1715 & -0.1208 & -0.0012 & -0.0532 & 0.0773 & -0.0906 \\
\hline & \multirow[t]{2}{*}{13.53} & \multirow[t]{2}{*}{1.04} & 220 & 2.66 & \multirow[b]{2}{*}{ Displacement } & 1.9847 & 2.0935 & 1.9473 & 1.5994 & 1.6465 & 1.8591 & 1.8458 \\
\hline & & & & & & 0.0000 & 0.1087 & -0.1462 & -0.3479 & 0.0471 & 0.2126 & -0.0133 \\
\hline & \multirow[t]{2}{*}{13.89} & \multirow[t]{2}{*}{2.05} & 920 & 11.11 & \multirow[b]{2}{*}{ Displacement } & 2.1249 & 2.1490 & 2.1080 & 2.0004 & 2.0741 & 1.7963 & 2.0572 \\
\hline & & & & & & 0.0000 & 0.0242 & -0.0411 & -0.1075 & 0.0737 & -0.2778 & 0.2609 \\
\hline & & & \multicolumn{3}{|c|}{ AVERAGE LENGTH } & 2.481051 & 2.503338 & 2.46432 & 2.369492 & 2.377102 & 2.413101 & 2.354271 \\
\hline & & & \multicolumn{3}{|c|}{ AVERAGE DISPLACEMENT } & 0 & 0.022288 & -0.03902 & -0.09483 & 0.00761 & 0.035998 & -0.05883 \\
\hline
\end{tabular}




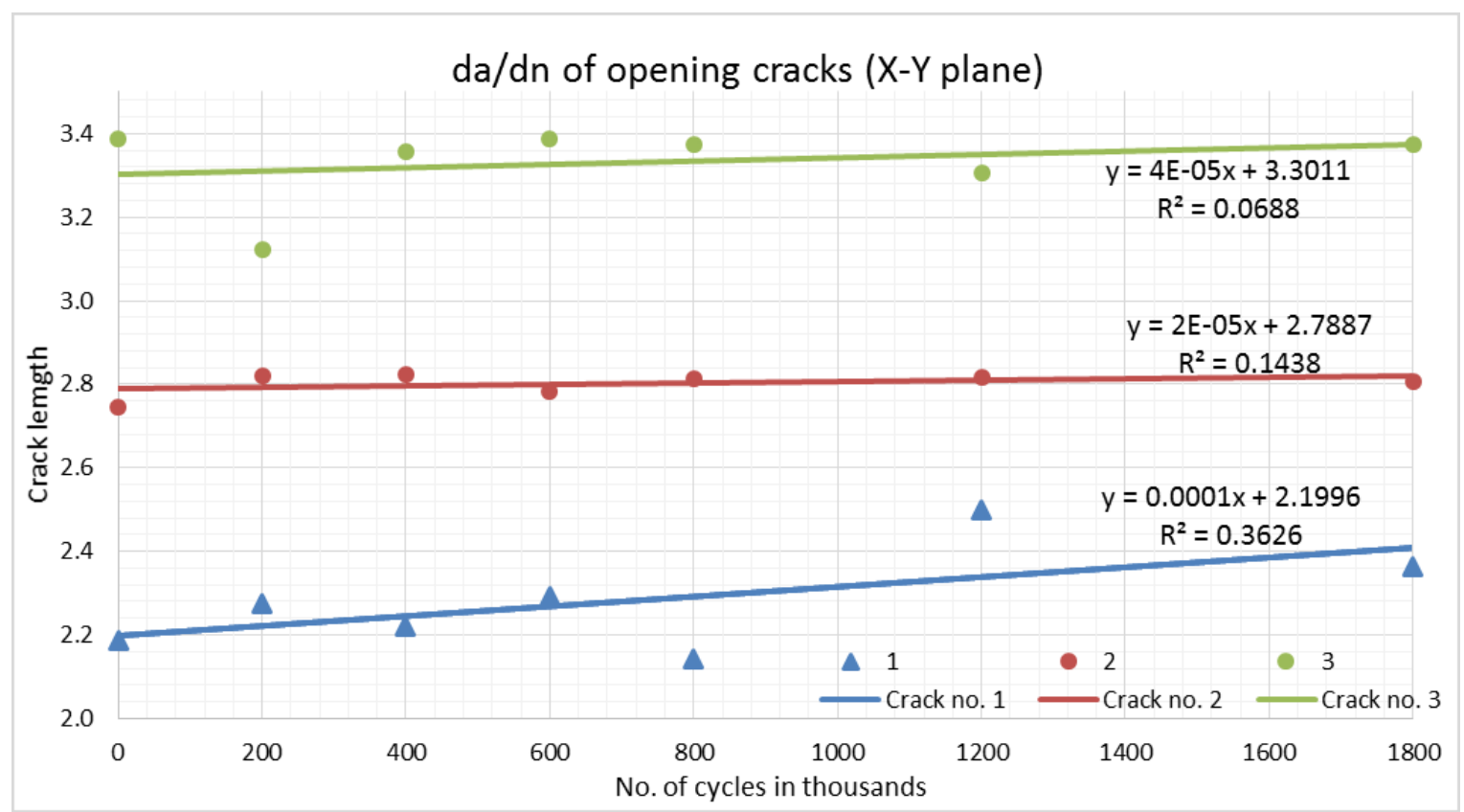

Figure 4.20: Fatigue crack growth of mode III opening cracks

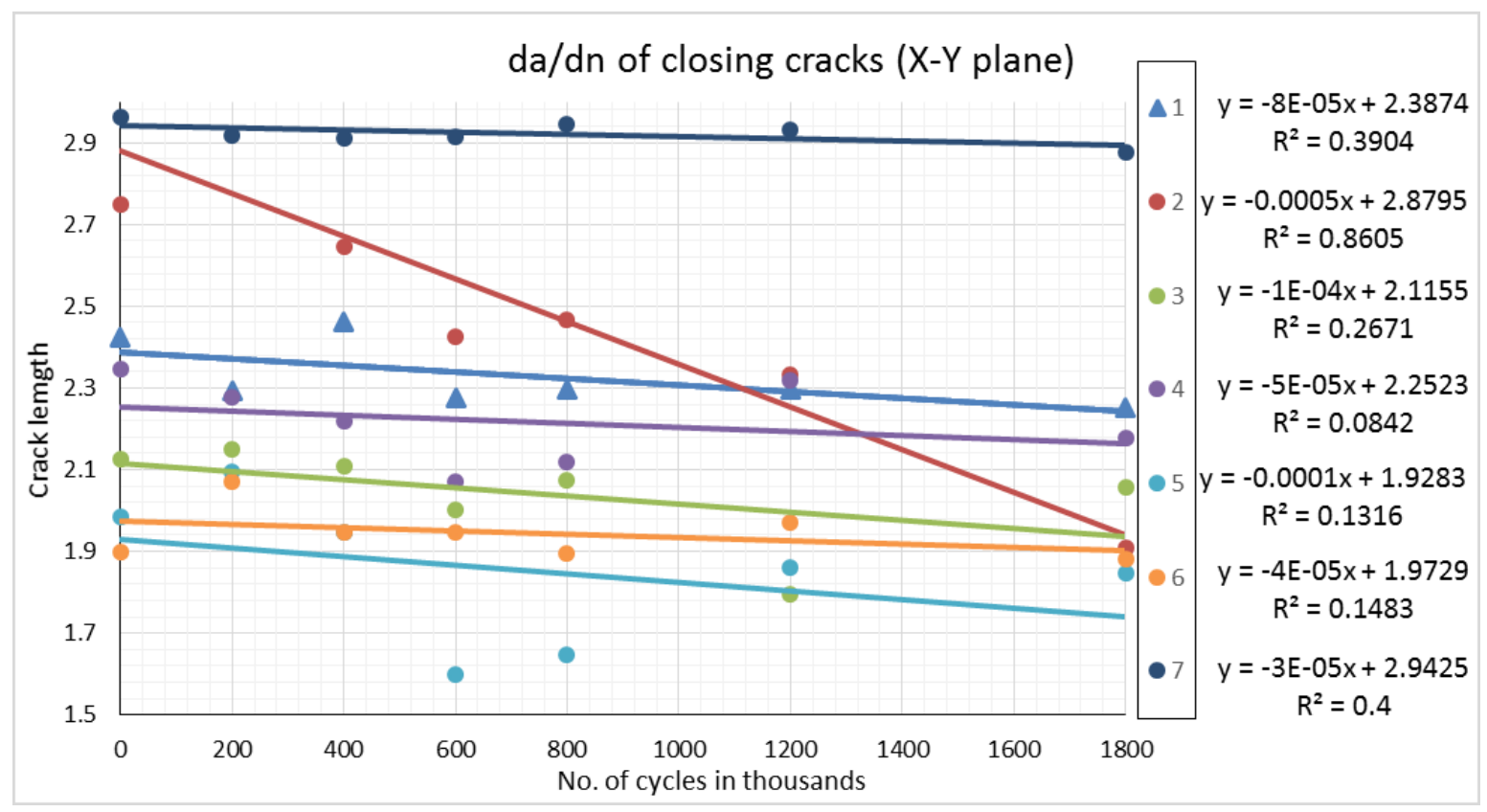

Figure 4.21: Fatigue crack growth of mode III closing cracks 


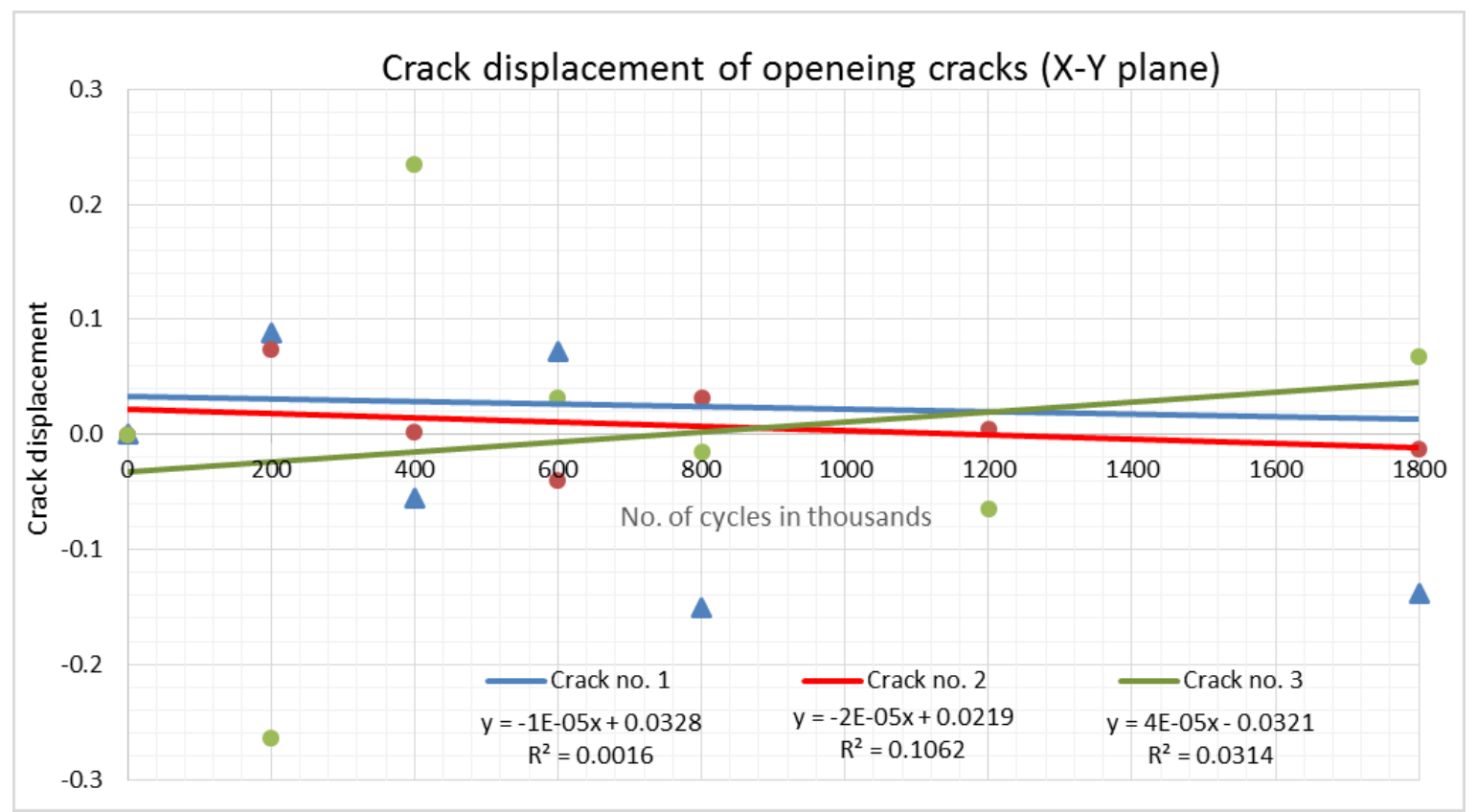

Figure 4.22: Crack displacements of mode III opening cracks

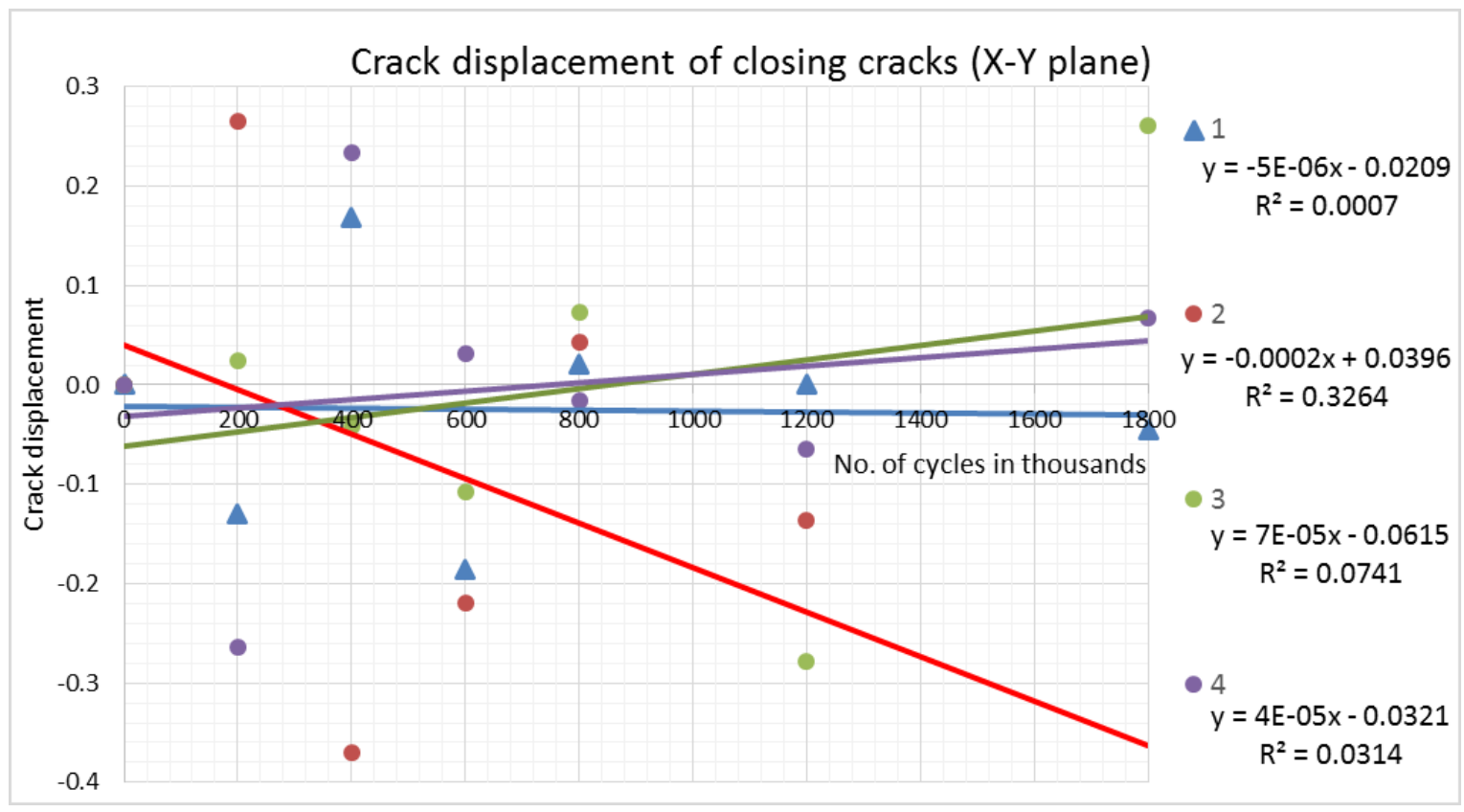

Figure 4.23: Crack displacements of mode III closing cracks 


\subsection{Fracture Energy}

Calculated values of fatigue crack growth rates in the 3 planes were directly implemented in LEFM Paris law in its general form, Equation (1), using values of constants published by Blanco et al. [112].

$$
\frac{d a}{d n}=C_{I, I, I I I} \Delta G^{m_{I, I I, I I I}}
$$

Equation 4.2

Where, $C_{I}=2.1, C_{I I}=C_{I I I}=0.12, m_{I}=5.09$ and $m_{I I}=m_{I I I}=4.38$ [112].

Strain energy release rate (fracture tougness), $\boldsymbol{G}$, was calculated for the 3 planes where the slope $d a / d n$ of the $\mathrm{X}-\mathrm{Z}$ plane was assumed to correspond to opening (mode I) fracture energy, $\boldsymbol{G} \boldsymbol{I}$, while the slope $d a / d n$ of the Y-Z plane addressed the in-plane shear (mode II) fracture energy, $\boldsymbol{G}_{\boldsymbol{I I}}$ and that of the X-Y plane corresponded to out-of-plane shear (mode III) fracture energy, $\boldsymbol{G}_{I I I}$, seen in Figures 4.24 and 4.25 .

Unit of constant $\boldsymbol{C}$ was in $(\mathrm{mm} / \mathrm{cycle})(\mathrm{N} / \mathrm{mm})^{-\mathrm{m}}$. Results were; $\boldsymbol{G}_{\boldsymbol{I}}=0.16208 \mathrm{~N} / \mathrm{mm}, \boldsymbol{G}_{\boldsymbol{I I}}=$ $0.19344 \mathrm{~N} / \mathrm{mm}$ and $\boldsymbol{G}_{I I I}=0.18265 \mathrm{~N} / \mathrm{mm}$.

All results are listed in Table 4.4. The outcome of this work was compared with previously published data of typical carbon/epoxy laminates having similar properties.

Blanco et al. [112] used a number of laminae of 5 or 20 symmetrical plies, corresponding to $\boldsymbol{\eta}=0.25$ or 1 (where $\boldsymbol{\eta}$ is the ratio between the thickness of the loaded and unloaded beams of the specimen, i.e. $h_{1} / h_{2}$ ), and Yang and Cox [106] used laminates of stacking sequence $[0 / 90 / 90 / 0]_{\mathrm{S}}$, while the results obtained from this work pertained to symmetric cross-ply laminate stacking sequence $\left[0^{\circ} / 90^{\circ} / 0^{\circ} / 90^{\circ}\right]_{\mathrm{s}}$. Results were of the same order of magnitude compared to the published data; however, considering the different fibre 
orientations, the observed variations could be justified. A comparison of results versus published data appear in Table 4.5.

Table 4.4: Summary of quantitative analysis

\begin{tabular}{|c|c|c|c|}
\hline \multirow[t]{2}{*}{ Orthogonal Planes } & $\mathrm{X}-\mathrm{Z}$ plane & Y-Z plane & X-Y plane \\
\hline & $\begin{array}{c}\text { Opening mode } \\
\text { (Mode I fracture) }\end{array}$ & $\begin{array}{l}\text { In-plane shear mode } \\
\text { (Mode II fracture) }\end{array}$ & $\begin{array}{l}\text { Out-of-plane shear } \\
\text { (Mode III fracture) }\end{array}$ \\
\hline VOI slice area (pixels $\mathrm{x}$ pixels) & $1972 \times 1370$ & $224 \times 1370$ & $1972 \times 224$ \\
\hline VOI slice area $(\mathrm{mm} \times \mathrm{mm})$ & $23.82 \times 16.55$ & $2.71 \times 16.55$ & $23.82 \times 2.71$ \\
\hline Number of slices & 224 & 1972 & 1370 \\
\hline Plane thickness (mm) & 2.71 & 23.82 & 16.55 \\
\hline Threshold method & Minimum & Triangle & Triangle \\
\hline Total number of detected cracks & 26 & 30 & 40 \\
\hline area of initial detected voids $\left(\right.$ pixel $\left.^{2}\right)$ & $5.0353 \mathrm{E}+05$ & $2.3215 \mathrm{E}+05$ & $6.1600 \mathrm{E}+05$ \\
\hline$\%$ area of initial detected voids & $0.0832 \%$ & $0.0389 \%$ & $0.1018 \%$ \\
\hline area of voids after $1.8 \times 10^{6}$ fatigue cycles (pixel ${ }^{2}$ ) & $5.1335 \mathrm{E}+05$ & $1.8974 \mathrm{E}+05$ & $5.4448 \mathrm{E}+05$ \\
\hline$\%$ area of voids after $1.8 \times 10^{6}$ fatigue cycles & $0.0848 \%$ & $0.0314 \%$ & $0.0900 \%$ \\
\hline$\%$ change in void content & $1.9202 \%$ & $-19.4911 \%$ & $-11.6204 \%$ \\
\hline Average initial crack length (mm) & 2.1180 & 1.7527 & 1.8744 \\
\hline Average final crack length (mm) & 2.1457 & 1.7105 & 1.7400 \\
\hline Observed da/dn slope & $1.9940 \mathrm{E}-04$ & $-9.0000 \mathrm{E}-05$ & $-7.0000 \mathrm{E}-05$ \\
\hline Paris law using Blanco's constants & $d a / d n=2.1 G_{I}^{5.09}$ & $d a / d n=0.12 G_{I I}^{4.38}$ & $d a / d n=0.12 G_{I I I}^{4.38}$ \\
\hline Strain energy release rate $\mathrm{G}_{\mathrm{I}, \mathrm{II}, \mathrm{III}}(\mathrm{N} / \mathrm{mm})$ & 0.16208 & 0.19344 & 0.18265 \\
\hline Modulus of Elasticity (MPa) & $E_{11}=120,000$ & $E_{22}=10,500$ & $E_{33}=10,500$ \\
\hline Maximum displacement (mm) & 0.0520 & 0.1920 & 0.1865 \\
\hline Maximum traction $(\mathrm{N})$ & 6.2369 & 2.0155 & 1.9585 \\
\hline
\end{tabular}

Table 4.5: Comparison of published and experimental data

\begin{tabular}{|c|c|c|c|}
\cline { 2 - 4 } \multicolumn{1}{c|}{} & \multicolumn{3}{c|}{ Strain energy release rate $(\mathrm{N} / \mathrm{mm})$} \\
\cline { 2 - 4 } \multicolumn{1}{c|}{} & $\mathrm{G}_{\mathrm{I}}$ & $\mathrm{G}_{\mathrm{II}}$ & $\mathrm{G}_{\mathrm{III}}$ \\
\hline Experimental results & 0.1621 & 0.1934 & 0.1827 \\
\hline Yang and Cox [106] & 0.3500 & 0.7000 & 0.7000 \\
\hline Blanco et al. [112] & 0.2600 & 1.0020 & $\mathrm{~N} / \mathrm{A}$ \\
\hline
\end{tabular}




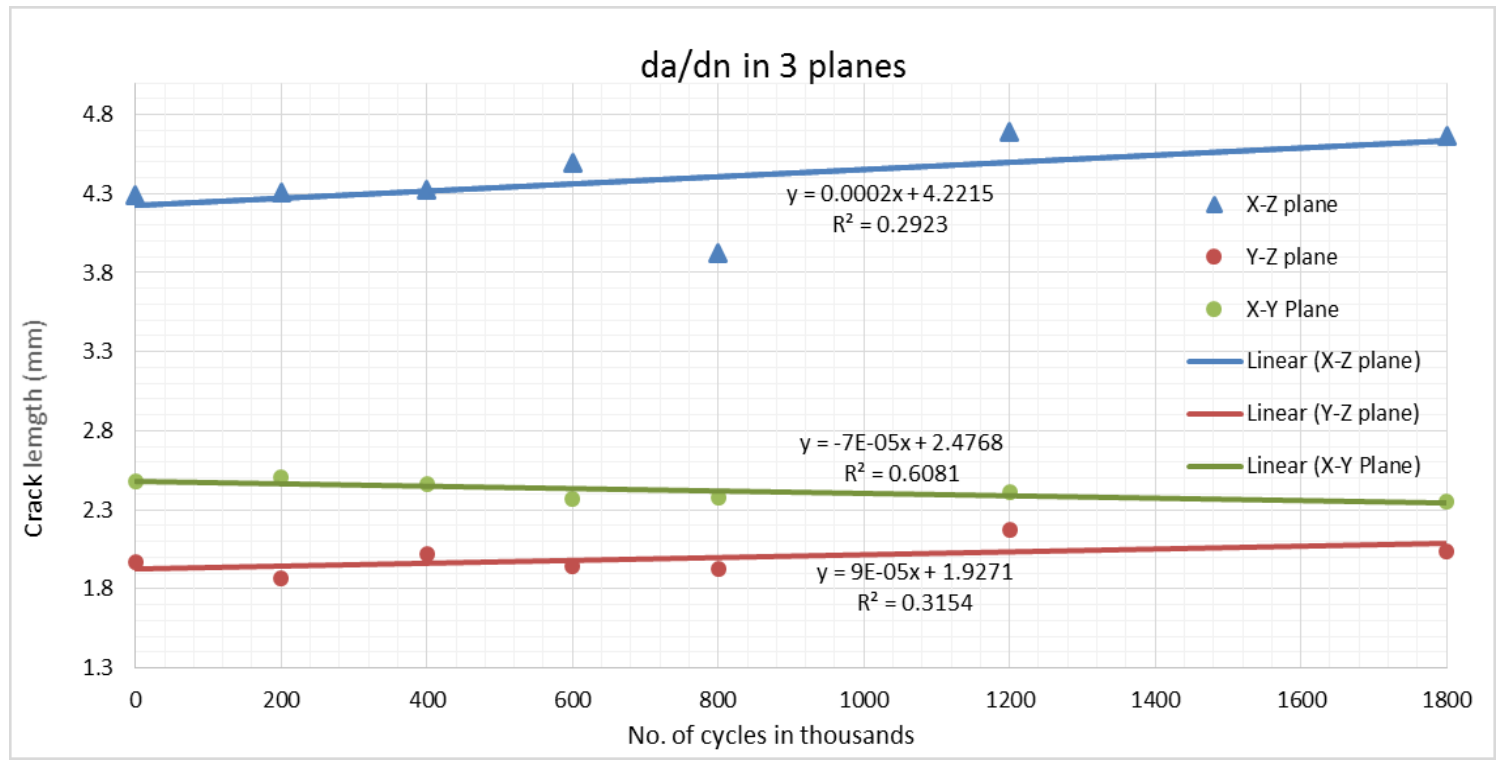

Figure 4.24: General trend of fatigue crack growth rate in the 3 orthogonal planes

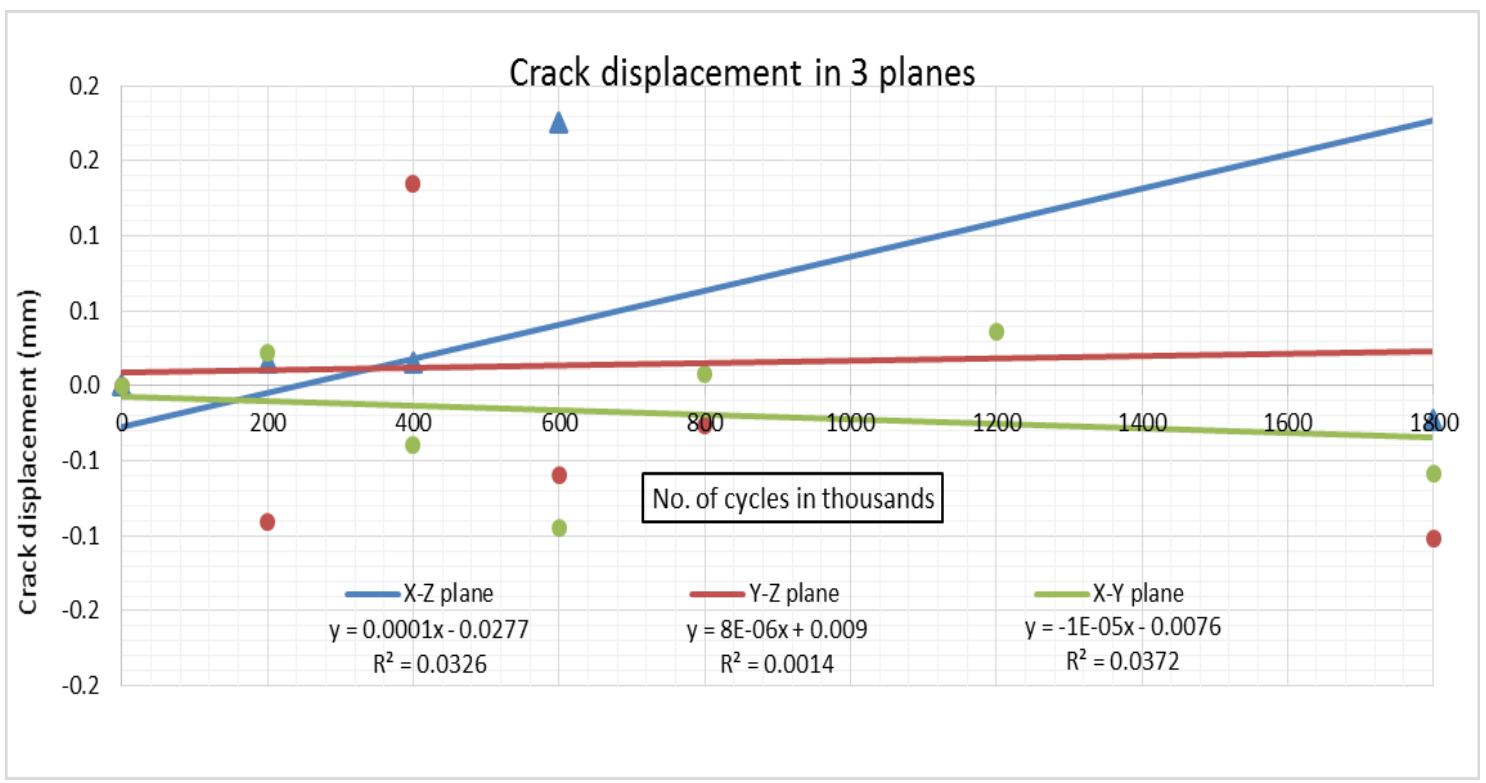

Figure 4.25: General trend of crack displacements in the 3 orthogonal planes

\subsection{Conclusion}

Ultra high-resolution microCT Skyscan 1173 successfully provided non-destructive probing of the internal damage propagation caused by constant amplitude fatigue loading in laminated CFRP composites of the type used in aircraft structures. Micro-meso data of distribution, frequency and geometry of the initially detected cracks and their progression 
over fatigue life were quantitatively analysed, through studying stacks of image sequences of virtual cross-sections in 3 orthogonal planes. Fatigue crack growth rates in the 3 planes were observed and used to calculate strain energy release rate, $\boldsymbol{G}$, by using LEFM laws. Their values were used to define traction-separation parameters for the 3 damage modes; opening, in-plane shear and out-of-plane shear. It was made possible for the first time, to the author's knowledge, to differntiate between in-plane shear and out-of-plane shear damage modes in terms of fracture toughness, maximum traction and maximum separation which were generally assumed to be identical in previous published literature. 


\section{Chapter 5:}

\section{Verification of Delamination}

\section{Variables}

\subsection{Introduction}

In Chapter 4 of this thesis, a non-destructive investigation of the through-thickness void content was described at the micro-meso scale using the high resolution microCT scanning. In the present chapter, a process developed to verify the characteristics of delamination featuring in-plane shear mode II and out-of-plane shear mode III (the predominant modes of fracture associated with delamination), is presented. Coupling between fracture modes I and II was neglected, although an opening mode I (normal displacement) was described by some authors such as the experimental work done by Zhang et al. [121] for determining the fatigue delamination growth rate under mixed I/II loadings.

In this chapter, results obtained from LEFM laws were verified by studying the propagation of 4 distinctive cracks. While damage varies in form from intra-ply matrix cracks and fibre rupture to inter-ply delamination, the latter was chosen to be the subject of this work being the most common form of damage observed in laminated CFRP. Although widely studied 
and results of MMB test for static loading [111] and fatigue loading $[112,113]$ experiments are available in literature, experimental work providing delamination variables specifically for laminated CFRP caused by fatigue loading is still very much an open area of investigation. A further motivation is that the nucleation of crack is known to more likely will initiate from the notch which is the location of stress concentration. Moreover, delamination FEA simulation features simplicity in assuming the crack path which must lie in an almost zero-thickness plane; between plies, so mesh sensitivity is not a requirement. Work introduced in this chapter was presented in SAMPE Conference [16].

Nonlinearity of damage behaviour in composites is associated with the interactions between simultaneous damage modes resulting in continuous alterations of crack paths relative to the redistribution of stress-strain mapping. Therefore, several approaches were introduced [122 - 119] including analytical tools used to account for the nonlinearity in stress-strain mapping featuring the damage behaviour in materials; composites in particular. Hence overcoming limitations associated with conventional LEFM [15]; Paris law which is more convenient in studying metals and another less common approach, VCCT [118], which assumes a self-similar crack propagation and requires a predefined crack path.

Nevertheless, coupled with continuous stress redistribution, a major problem is encountered with such applications that is the necessity of very fine mesh at the crack tip so as to capture the response of numerous discretized cohesive elements. Mesh dependency is circumvented either by defining the crack path a priori or by adopting special mesh strategies; such as enrichment of the crack-tip nodes in the partition of unity concept. 


\subsection{Image Processing}

The notched laminated CFRP sample previously studied in Chapter 4 was re-investigated at 5 intervals. Following a similar procedure, image processing involved virtual slicing throughout the thickness of each dataset of images acquired from microCT scans for each of the 5 fatigue intervals; $0,200,000,400,000,600,000$ and 800,000 cycles. Segmentation was performed by applying an automatic threshold method for separating the voids, and then statistical analysis of segmented images was carried out to evaluate the void growth throughout the fatigue life.

Voids were classified according to their size in pixel ${ }^{2}$, circularity and consistency, i.e. occurrence in at least 3 successive slices. Preliminary visual assessments suggested that delamination defects could be considered if the void area exceeded 1000 pixel $^{2}$, and could be further suspected if the void area ranged from $700 \mathrm{pixel}^{2}$ to $1000 \mathrm{pixel}^{2}$. Circularity of the delamination defects was assessed as being less than 0.25 for the longitudinal shaped defect lying adjacent to fibrous plies in the negligible-thickness resin layer. Hence, all voids of areas below 700 pixels $^{2}$ and circularity above 0.25 were neglected. Moreover, voids that did not appear in at least 3 successive cross-sections were not considered.

For calculating the length of delamination cracks, a statistical study was conducted of the average delamination thickness leading to an estimation of 10 pixels $(0.12 \mathrm{~mm})$ thickness. Assuming an almost rectangular delamination crack, the length of cracks were calculated by dividing the detected void areas by 10 . Hence the crack tip displacement (difference in subsequent crack length) relative to the fatigue life was traced. The above procedure was performed manually for the available resources at this point of research. 
Generally, void content was calculated as a percentage of the total area of cracks in pixel $^{2}$ as summed up throughout the dataset sequence relative to the total area of stacked images. Cracks were classified as above 0 (all voids), 500 pixel $^{2}, 700$ pixel $^{2}, 1000$ pixel $^{2}$ up to infinity for each fatigue phase. Results are plotted in Figure 5.1, showing that an initial void content of $0.24 \%$ was observed. The percentage dropped in the 400,000 and 600,000 cycle's phases, contrary to the expected trend. This may be attributed either to the microscopic rearrangement of fibrous plies as fatigue loading continues thus closing small voids due to re-orientation, or otherwise to errors in applying the threshold levels leading to underestimation of void content. Investigating a larger set of samples is proposed for a comprehensive analysis to verify the correct threshold limits.

Although fatigue stress loading used in this testing was about $50 \%$ of the maximum tensile strength of this material, an almost steady void content of cracks over $500 \mathrm{pixel}^{2}, 700 \mathrm{pixel}^{2}$ and 1000 pixel $^{2}$ were observed throughout fatigue life phases. Further fatigue load testing is required to verify the number of fatigue cycles resulting in observed crack progression.

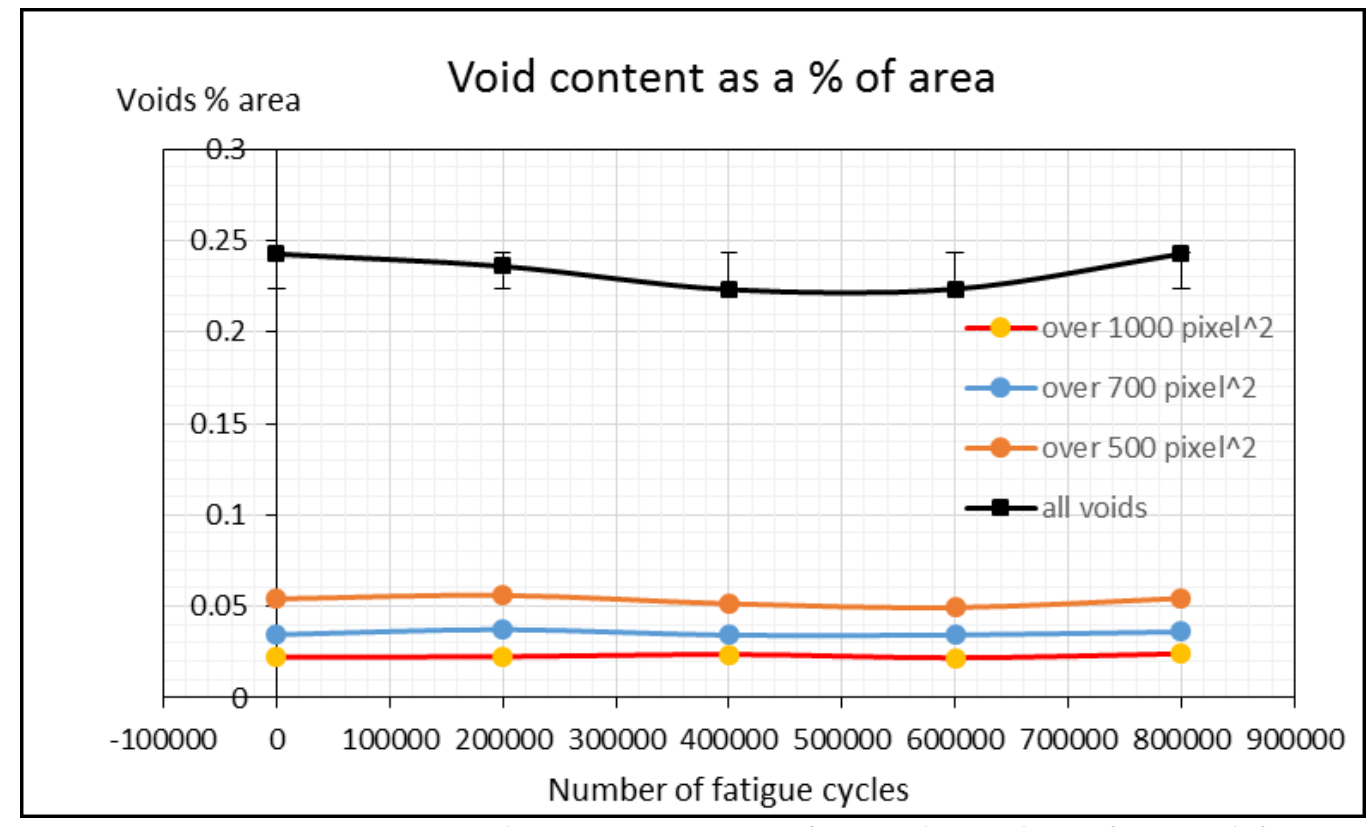

Figure 5.1: Void content as a \% of area throughout fatigue life 


\subsection{Statistical Analysis of Void Content}

Material lay-up was oriented such that the prepreg layers are aligned in the X-Z plane. Virtual cross-sections were stacked in each of the $\mathrm{X}-\mathrm{Y}$ plane, $\mathrm{Y}-\mathrm{Z}$ and $\mathrm{X}-\mathrm{Z}$ planes for all phases. However, this work considered studying the delamination mode only. Therefore, for studying the delamination mechanism, differentiation between the in-plane shear and the out-of-plane shear modes associated with the delamination cracks was performed through probing the 2 perpendicular planes; Y-Z (sagittal) and X-Y (transverse) simultaneously. The X-Z (coronal) plane was not studied owing to that the in-plane voids pertained to fibre breakage and matrix cracks rather than delamination.

The in-plane shear was traced by assessing the crack growth in the $Y-Z$ plane such that the cracks were detected adjacent to the cross-ply layers and parallel to the direction of axial fatigue loading. Further change in crack length reflects an in-plane shear stress being relatively elevated. On the other hand, for the crack growth traced in the X-Y plane, i.e. growing perpendicular to the fatigue loading, it is said to be experiencing an out-of-plane shear stress elevating relative to the crack displacement. Analysis involved calculating the length in mm of every detected crack of area over 1000 pixel $^{2}$ and circularity below 0.25 . Plots of the weighted trends of crack length as detected along $\mathrm{Y}$ axis (in $\mathrm{Y}-\mathrm{Z}$ plane) and along $\mathrm{X}$-axis (in X-Y plane) are seen in a series of graphs in Figures 5.2-5.11, illustrating the phases of fatigue life. The heights of these cracks were plotted on a secondary axis on each graph. The value of $\mathrm{Y}$ in the $\mathrm{Y}-\mathrm{Z}$ plane or $\mathrm{X}$ in the $\mathrm{X}-\mathrm{Y}$ plane normally corresponds the starting location of the crack as measured from the origin. However, upon rotating the plane of investigation, the origin is shifted, hence there is an expected shift in locations that is accounted for by subtracting the updated location from the axis length. 


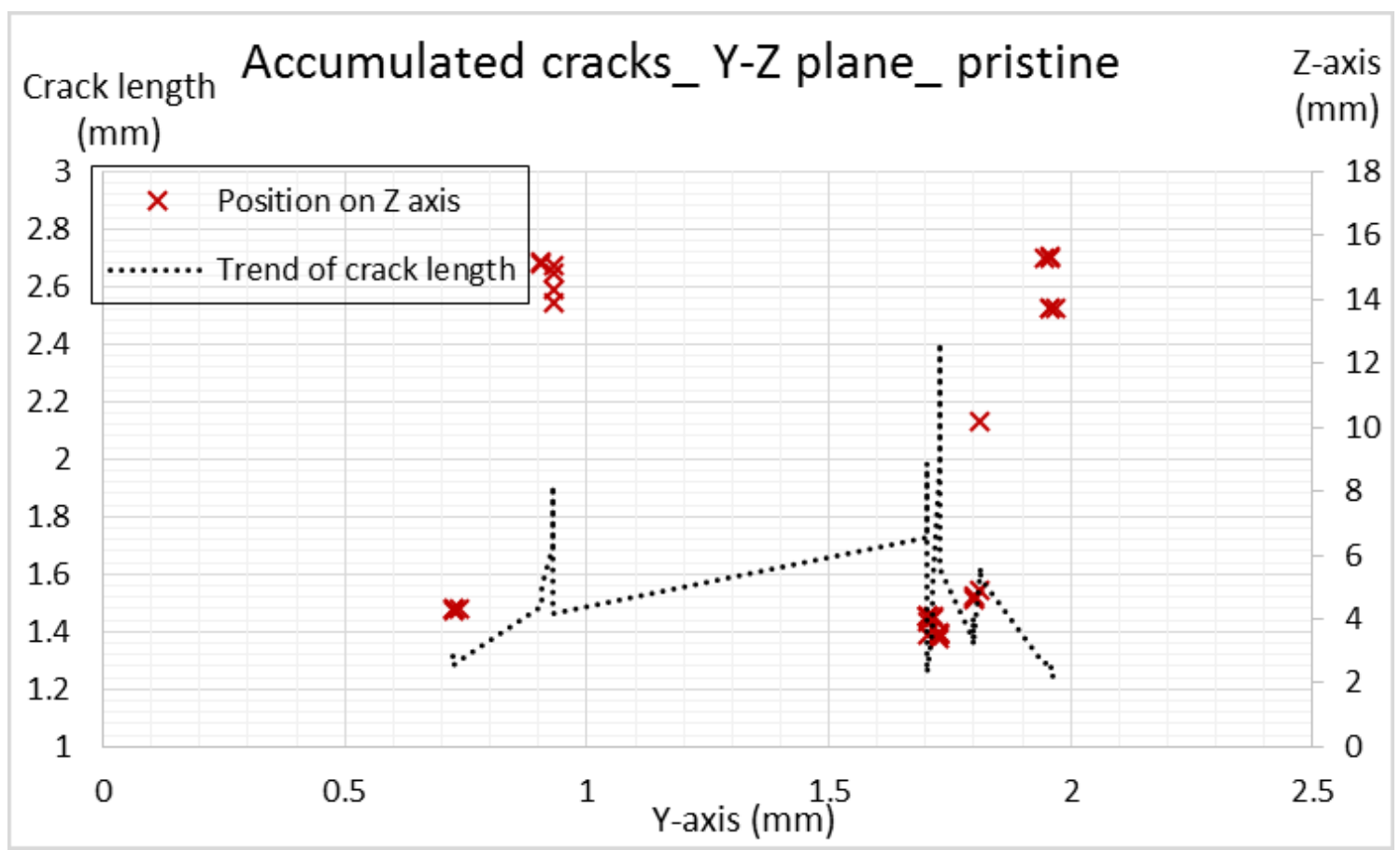

Figure 5.2: Accumulated cracks in Y-Z plane, pristine state

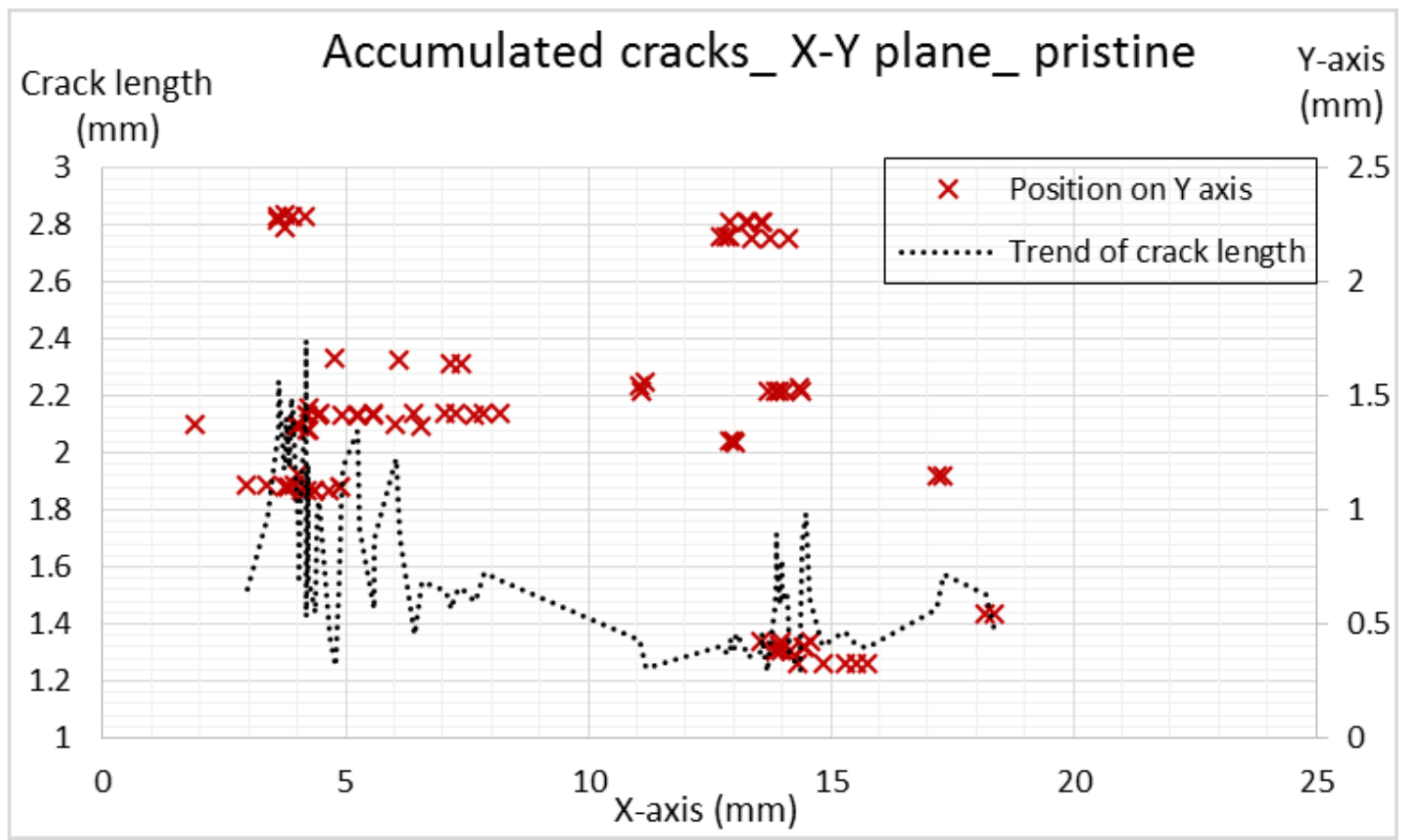

Figure 5.3: Accumulated cracks in $X$-Y plane, pristine state 


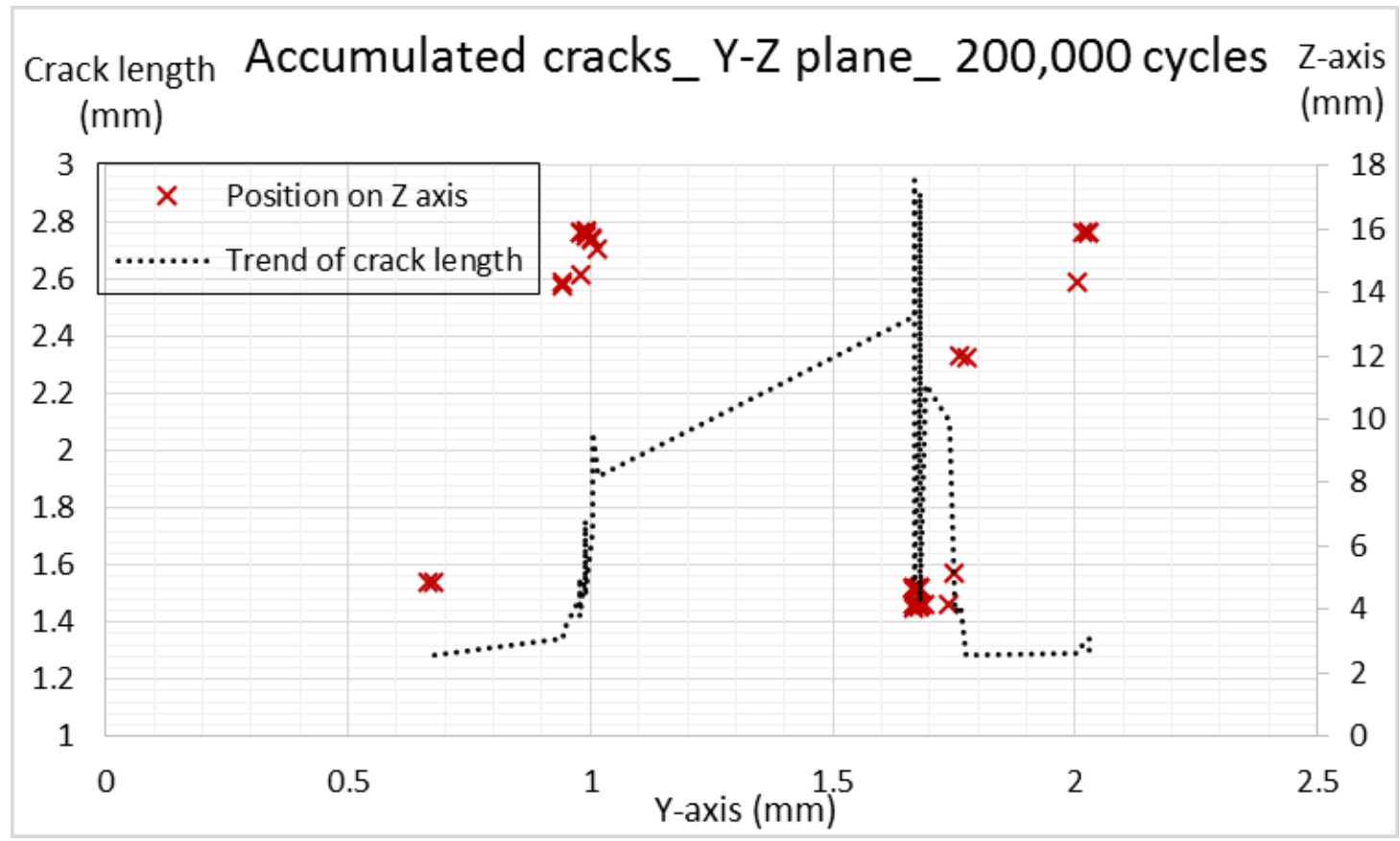

Figure 5.4: Accumulated cracks in Y-Z plane, 200,000 cycles

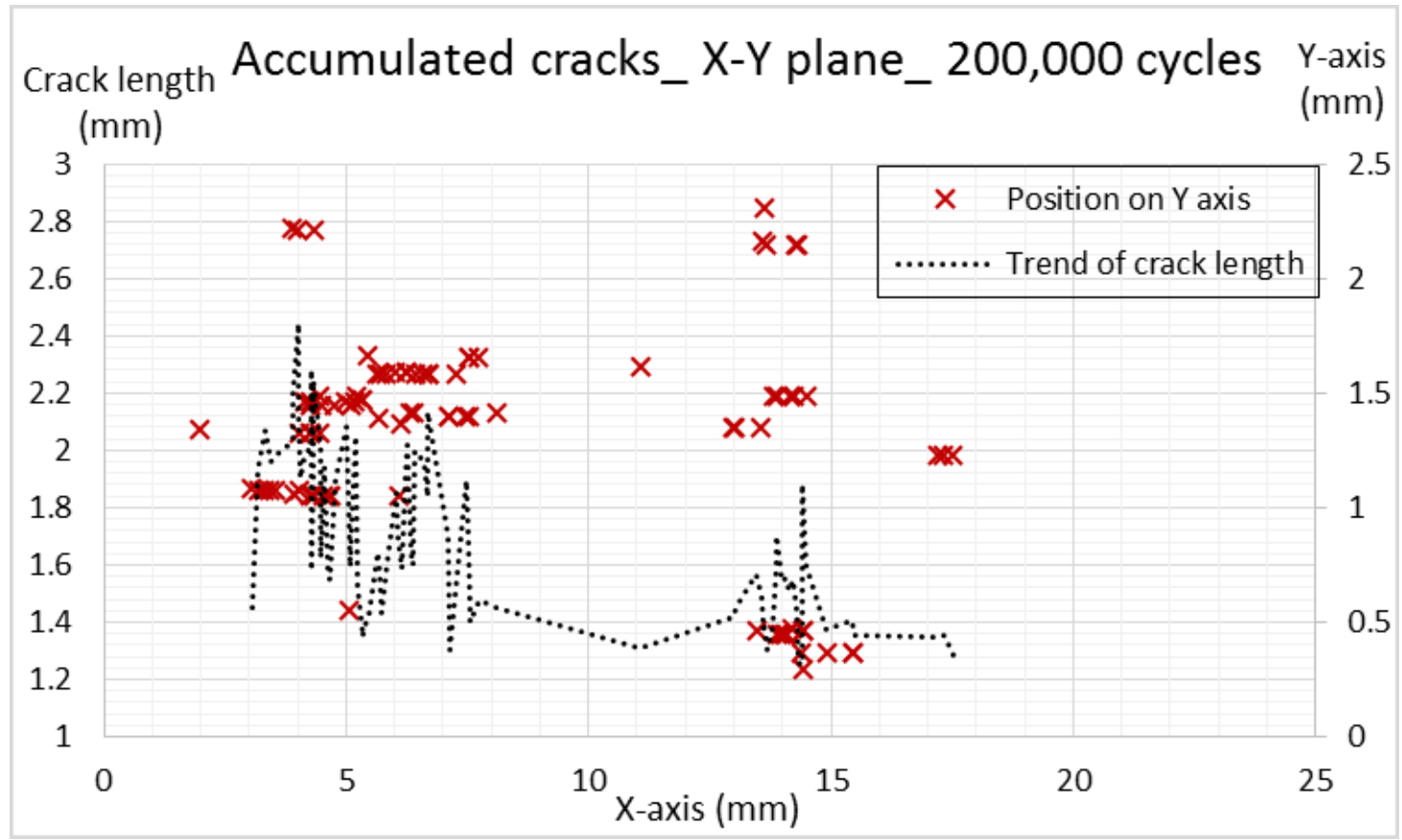

Figure 5.5: Accumulated cracks in X-Y plane, 200,000 cycles 


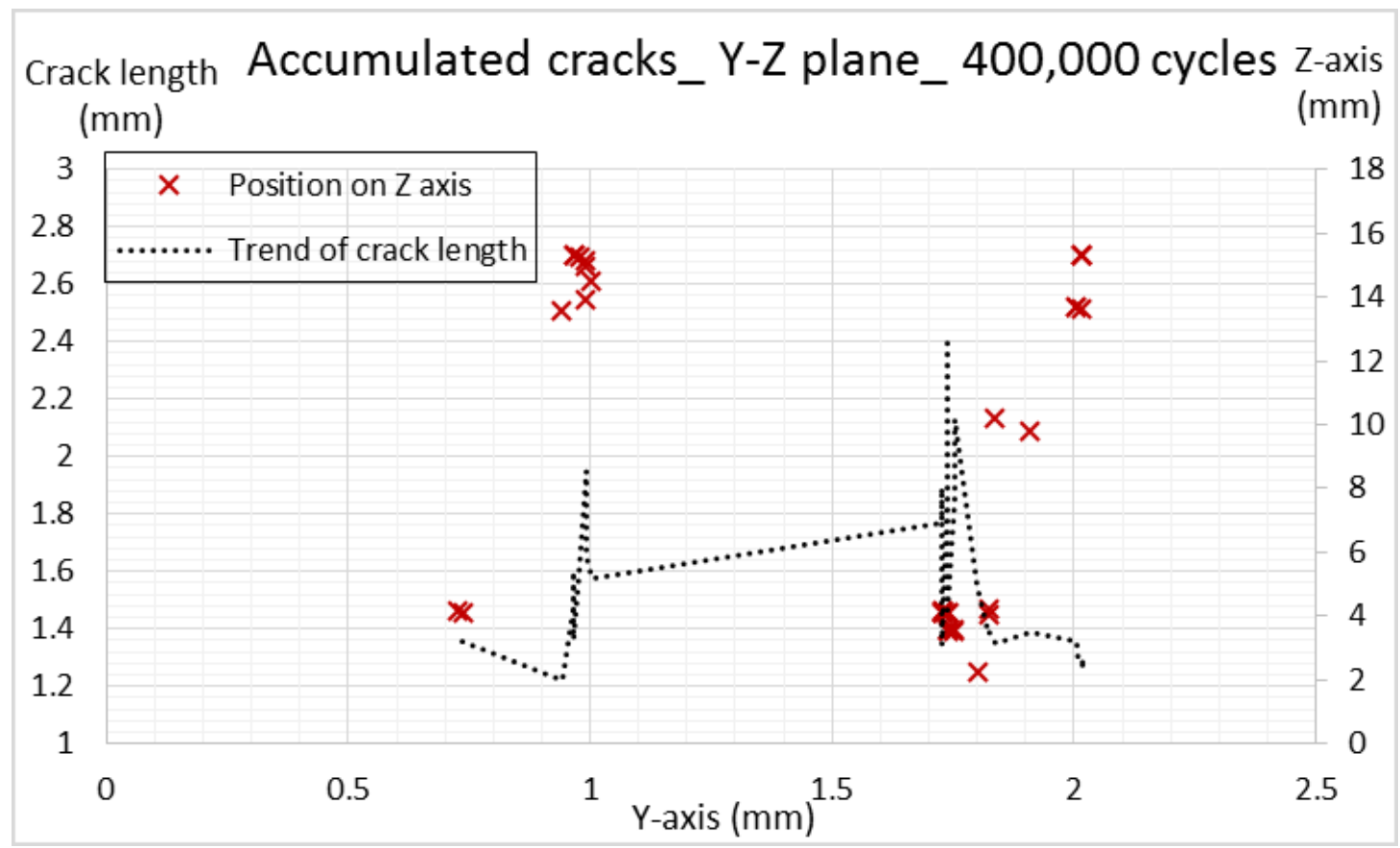

Figure 5.6: Accumulated cracks in Y-Z plane, 400,000 cycles

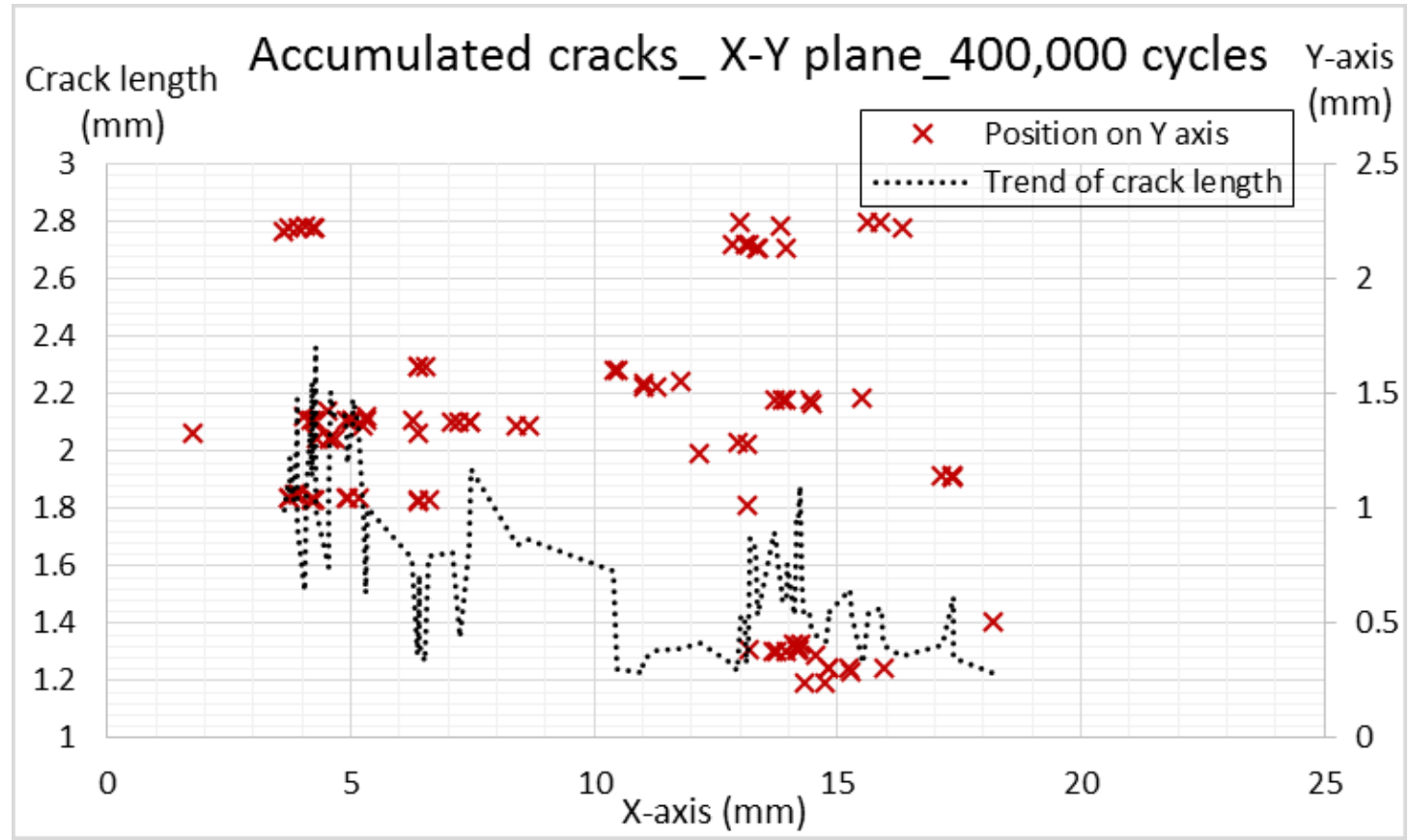

Figure 5.7: Accumulated cracks in X-Y plane, 400,000 cycles 


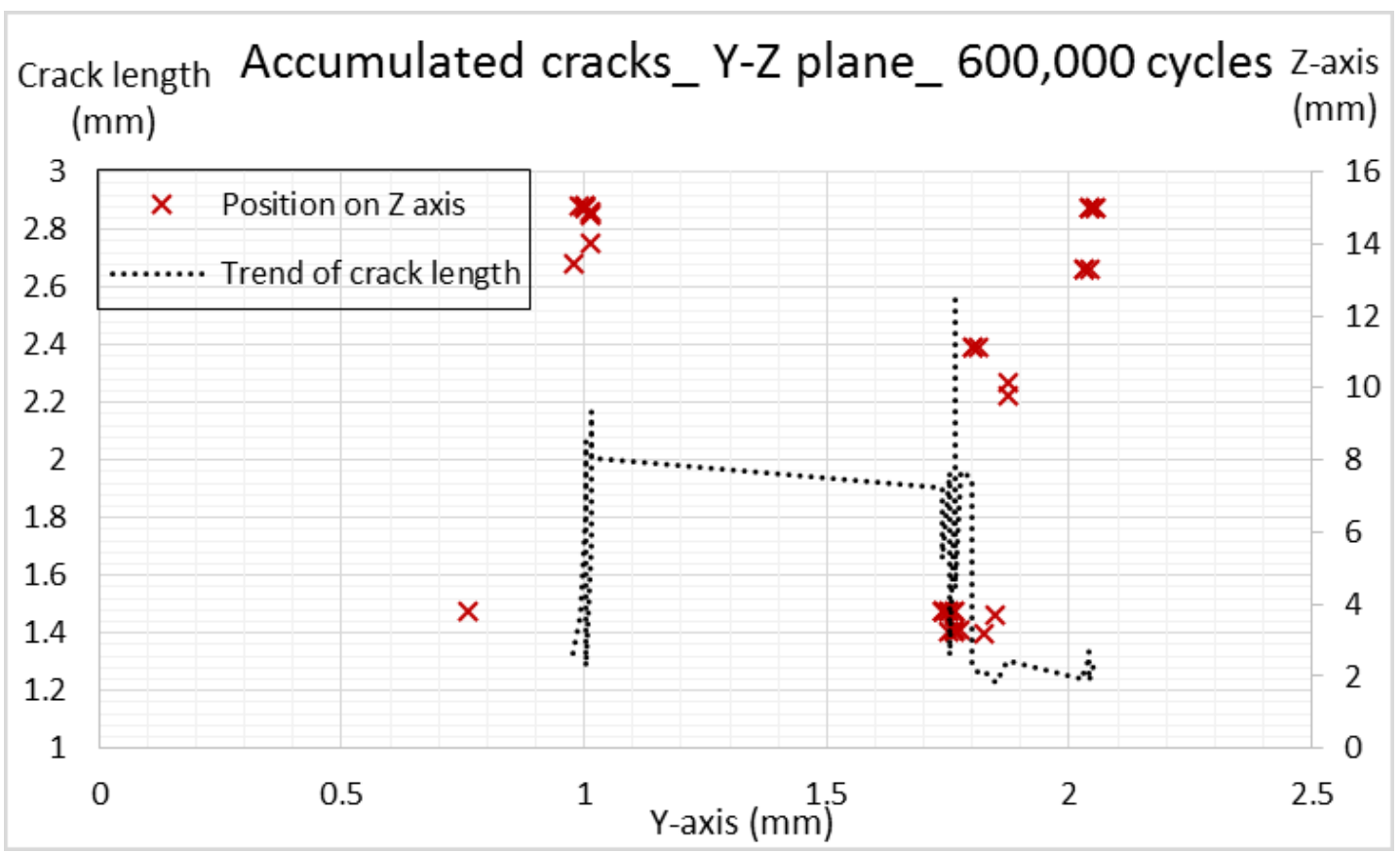

Figure 5.8: Accumulated cracks in Y-Z plane, 600,000 cycles

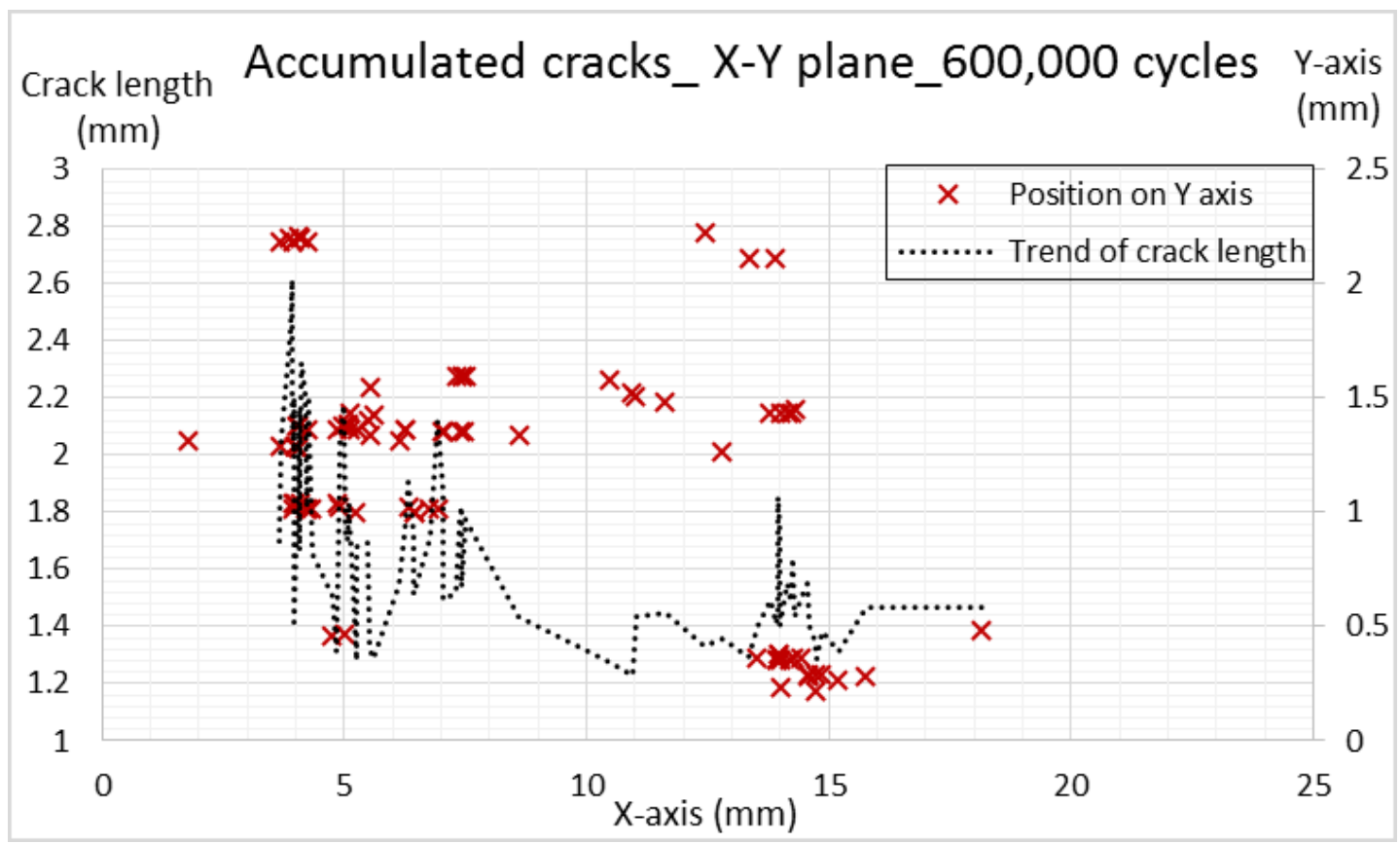

Figure 5.9: Accumulated cracks in X-Y plane, 600,000 cycles 


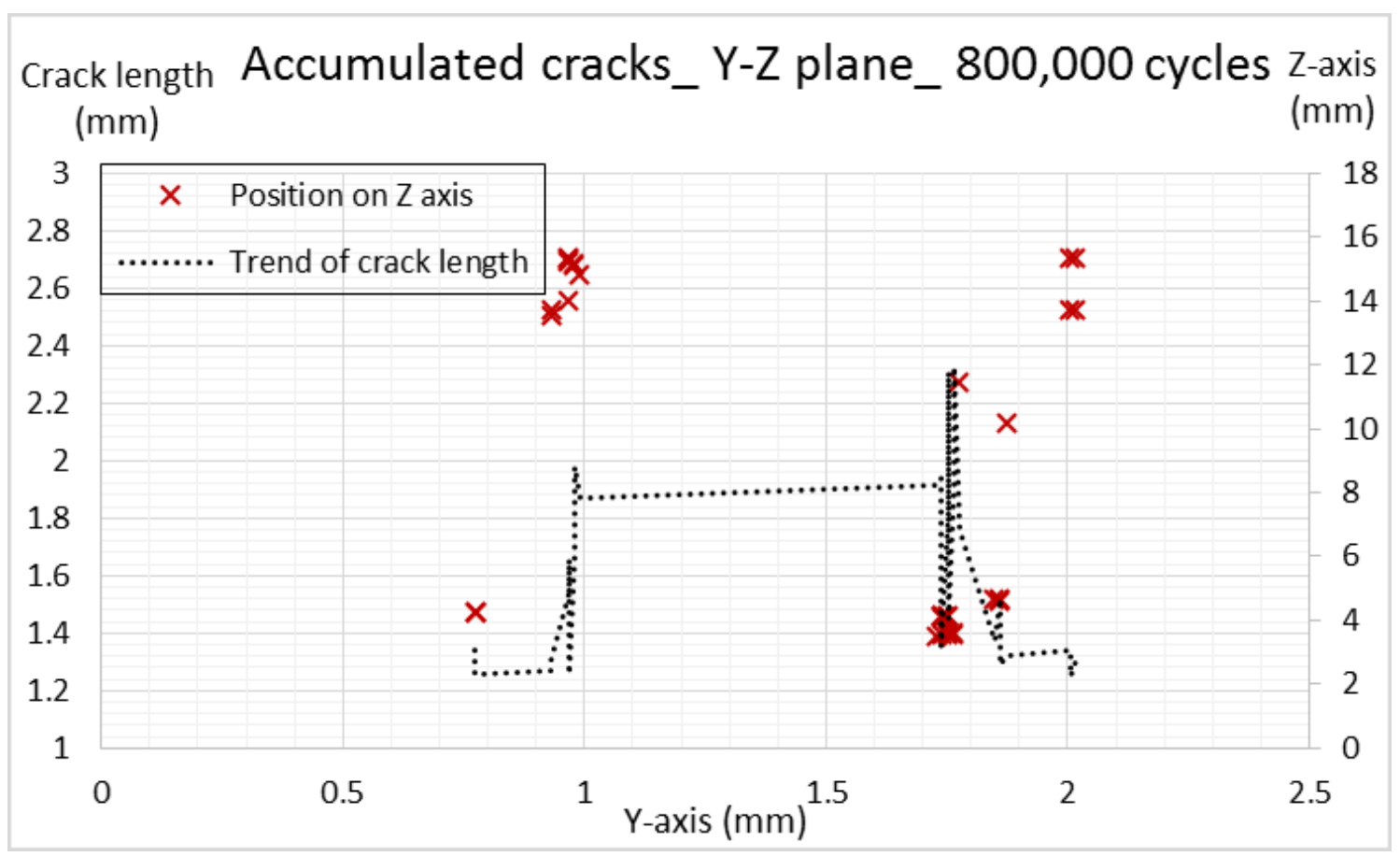

Figure 5.10: Accumulated cracks in Y-Z plane, 800,000 cycles

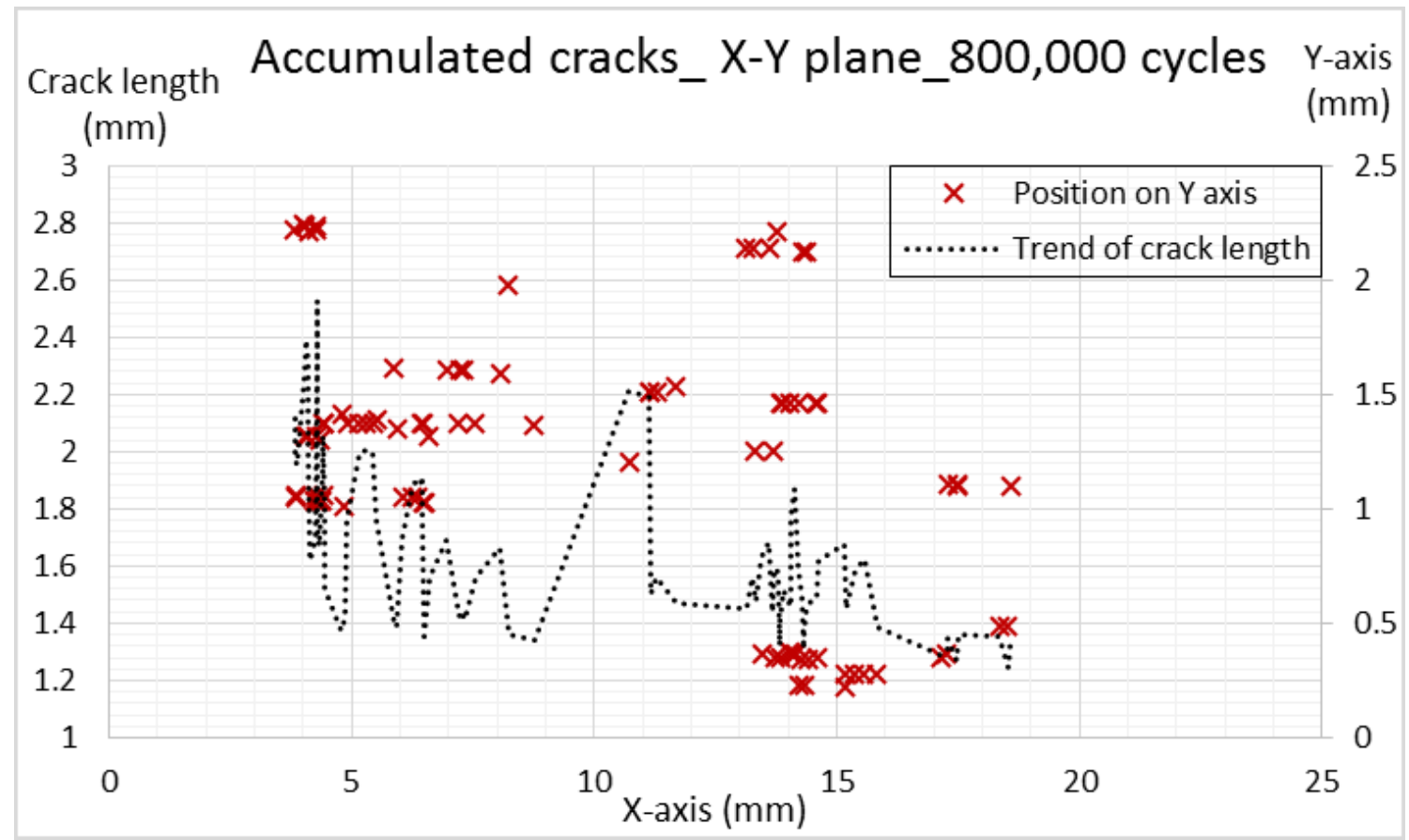

Figure 5.11: Accumulated cracks in X-Y plane, 800,000 cycles 
An overall count of number of cracks relative to number of cycles in the 3D sample and average crack length are shown in Table 5.1. Average crack length showed very slight rise from $1.58 \mathrm{~mm}$ to $1.67 \mathrm{~mm}$ by reaching 800,000 fatigue cycles with a steady crack count.

Table 5.1: Change of crack length in sample with number of fatigue cycles

\begin{tabular}{|l|ccccc|}
\cline { 2 - 6 } \multicolumn{1}{c|}{} & 0 & 200,000 & 400,000 & 600,000 & 800,000 \\
\hline Sum of total cracks lengths $(\mathrm{mm})$ & 142.36 & 149.11 & 155.63 & 141.67 & 151.92 \\
Total number of cracks in 3D sample & 90 & 89 & 96 & 85 & 91 \\
\hline Average crack length $(\mathrm{mm})$ & 1.58 & 1.68 & 1.62 & 1.67 & 1.67 \\
\hline
\end{tabular}

From analyzing the graphs listed in Figures 5.3 -5.12, and comparing plots to the collected data, it has been determined to trace the progression of 4 distinct cracks which had been repeatedly spotted in statistical data and visualized in successive virtual slices. These cracks initially existed in the pristine sample with a length range from $1.86 \mathrm{~mm}$ to $2.58 \mathrm{~mm}$ as observed in the Y-Z plane whereas length ranged $1.28 \mathrm{~mm}$ to $2.45 \mathrm{~mm}$ as seen in the transverse X-Y plane. Other smaller cracks were ignored either because they were not consistent or they were too small to count as delamination defects. The slices containing the traced cracks were spotted on the statistical data to determine their position on the image series where successive virtually sliced images were 1 pixel apart from each other. Hence the through-thickness positions were calculated in $\mathrm{mm}(1$ pixel $=12.08 \mu \mathrm{m})$. Cracks were spotted at $3.59 \mathrm{~mm}, 4.31 \mathrm{~mm}, 5 \mathrm{~mm}$ and $5.74 \mathrm{~mm}$ along the X-axis and their heights were $3.31 \mathrm{~mm}, 9.01 \mathrm{~mm}, 10.04 \mathrm{~mm}$ and $14.07 \mathrm{~mm}$ along the Z-axis.

The same positions were traced in successive fatigue life intervals in both planes, and the length of these cracks were recorded throughout. Collected data are listed in Tables 5.2 and 5.3. As previously suggested, the crack growth in the $\mathrm{Y}-\mathrm{Z}$ plane was attributed to in-plane shear progression while the crack growth in the X-Y plane was correlated to the out-of- 
plane shear progression. Results show very slow crack growth in both modes for all 4 cracks.

Table 5.2: Progression of crack length in the in-plane shear plane

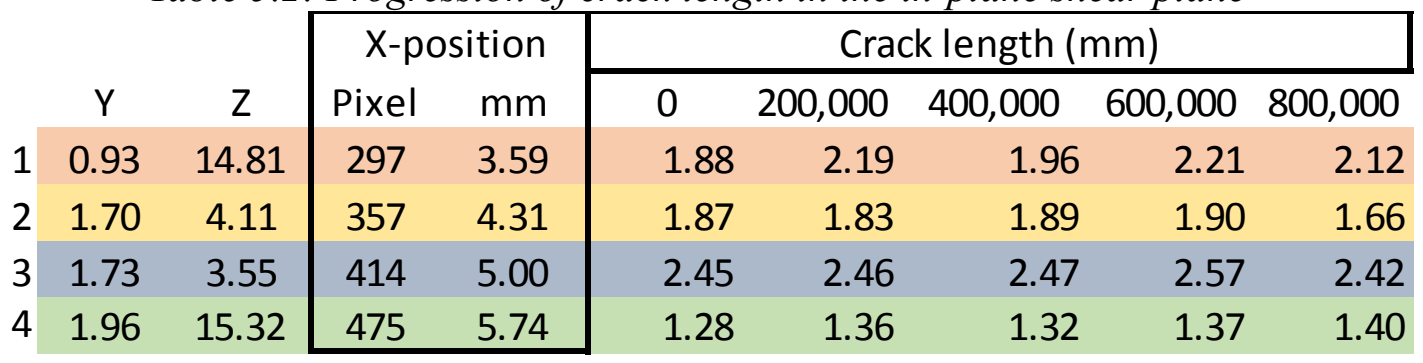

Table 5.3: Progression of crack length in the out-of-plane shear plane

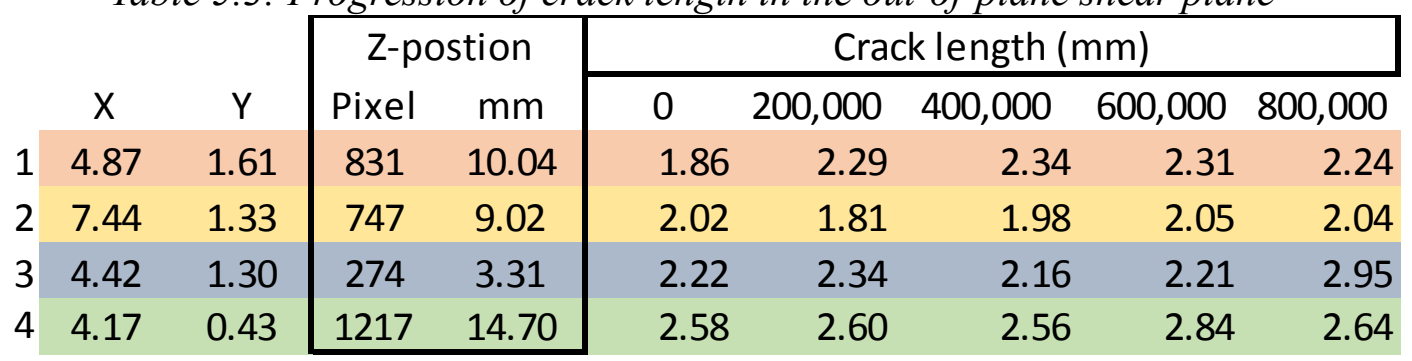

The cracks are visualized in Figure 5.12, which shows a 3D reconstructed volume rendered image of the volume of interest being studied, while the opacity was adjusted to qualitatively assess the crack content. Numerous cracks were detected but further quantitative assessment was not possible without slicing.

A link can be established between the LEFM law and traction-separation mechanism; where the peak stress in the traction-separation response corresponds to the onset of nonlinear crack propagation and the total area under the curve corresponds to the fracture toughness (or stress intensity factor) introduced in Paris law [114, 106 and 123]. The complete form of resulted data could be used to define an expression of the crack growth in terms of change of crack length relative to the number of fatigue cycles, $\mathrm{da} / \mathrm{dn}$. Hence the Paris type linear relation between the crack growth and fracture toughness can be made 
available (referring to work in Chapter 4) by implementing law coefficients available in open literature. Knowing 2 parameters allows for plotting the traction-separation response. In this work, fracture toughness and the displacements relative to the onset of shear stresses in both in-plane and out-of-plane modes were extracted form experimental micro-meso analysis, thus the bi-linear curve can be plotted by implementing a fatigue degradation factor.

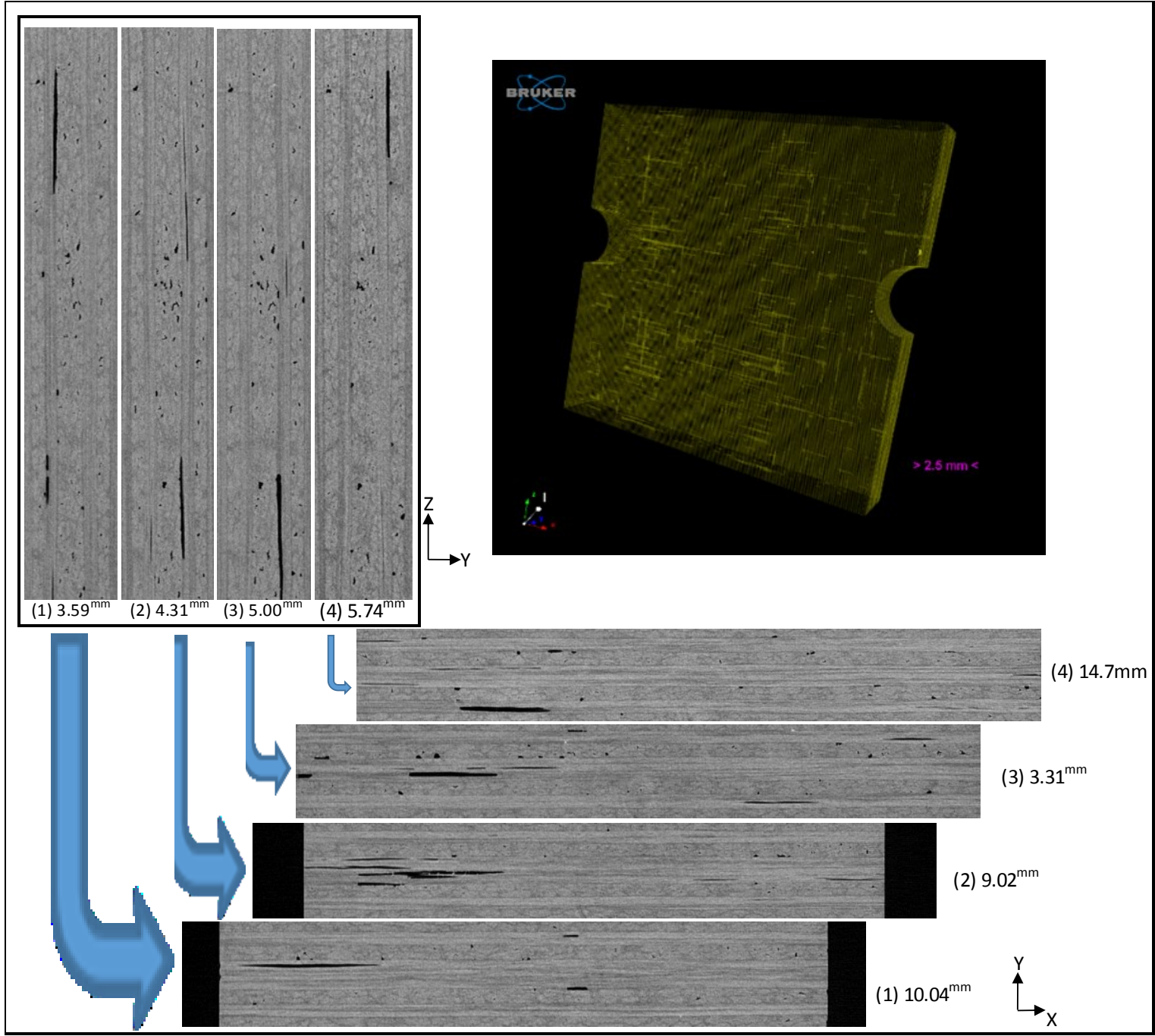

Figure 5.12: 4 cracks located in the Y-Z plane (upper left), and the same cracks were traced in the X-Y plane (under), with their through-thickness position defined for each. (Upper right) A $3 D$ reconstructed rendered volume of the VOI under investigation 


\subsection{Conclusion}

Destructive experimental procedures followed to determine fatigue growth parameters was avoided by using non-destructive microCT technique which proved powerful in providing detailed quantitative information of real cracks and their progression relative to the fatigue life. However, destructive testing is still ultimately needed for verifying the non-destructive results. Literature reviews had always assumed a similar behaviour of traction-separation response for both in-plane and out-of-plane shear modes due to lack of experimental results and difficulty associated with differentiating both modes. Aided by the non-destructive scans and by statistically analyzing virtual slices, it was possible to capture the crack displacements in either modes separately. Verification of traction-separation responses was accomplished by following the link between the extracted data and LEFM type laws. 


\section{Chapter 6:}

\section{Modelling in Abaqus}

\subsection{Introduction}

In this chapter, the results of a series of 4 simulation models addressing different test cases were created in the commercial FEA tool, Abaqus software are presented. The objective was, on the one hand verifying theoretical material properties, and on the other, validating the experimental results obtained from analyzing microCT scans.

To distinguish both terms, Roache [124] differentiated between "verification" which deals with mathematics for assuring the accuracy of code or input data and "validation" which confirms the representation accuracy of that model. Correspondence of verification to physical reality could be poor, hence leading to the necessity of validation which refers to the real experimental data as a standard.

In other words, verification answers the question: "are we solving the equations correctly" compared to the question of "are we solving the correct equations" answered by validation. 
Analysis of microCT scans conformed to the proposed procedure detailed in Chapter 3 and discussion of the results as detailed in Chapters 4. Noting that this software was introduced for the purpose of assessing its capabilities and limitations, it was not expected for the created models to adequately address the multi-mode damage behaviour as observed from the experiments. Rather, single-mode damage was modelled in each case as well as a genuine constructed VOI of microCT scans was imported and studied for comparing the 3D simulations versus observations.

Starting with verifying the theoretical data of stress concentration factor (SCF) of a notched specimen, model case 1 was used to compare simulated to theoretical results of SCF given the known material properties. Model case 2 was designed to study the stress intensity factors (SIFs) of an occurring crack. A static crack was modelled in the critical notch area and $J$ integrals were plotted versus time for several loading conditions. For both model cases 1 and 2, a 3D sample was designed having the same geometry and material properties as that of the experimental sample. However, in case 1, the geometry was modelled as an 8-ply composite, whereas in case 2 , the material was assumed to be isotropic due to a limitation in Abaqus software which precludes modelling cracks in layered materials.

In model case 3 , a crack was studied in a 3D sample of assumingly isotropic material. Experimental data of material properties and 3-mode fracture energies were used for model inputs.

In model case 4, Abaqus simulation modeling of cracks was validated versus analysis of microCT scans. A relatively small VOI near the notch was defined on the microCT scanned sample, segmented and meshed using ScanIP software [104] and then imported into 
Abaqus. Voids contained in the VOI were separated in a non-exportable mask using the ScanIP software [104], but the nodes constructing these voids were exported into Abaqus in a set used for defining the crack location of the assembly. A summary of these models is presented in Tables 6.3 and 6.4 at the end of this chapter.

\subsection{Simulation Models}

Simulation was performed using Abaqus/CAE suite version 6.14. Dimensions of the experimental sample detailed in Chapter 2 were realized. However, due to its vertical and horizontal symmetry, only one quarter of the sample was created to save computational time. Asymmetric boundary conditions were assumed on $\mathrm{X}$ and $\mathrm{Y}$ axes, Figure 6.1 whereas loading conditions and cracks parameters varied according to models designs.
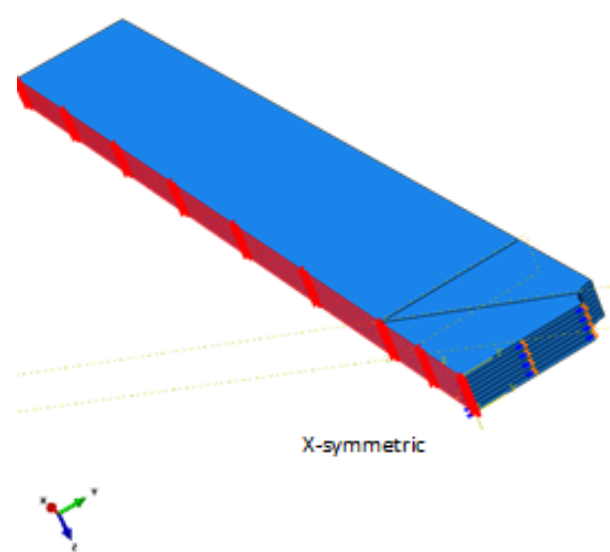

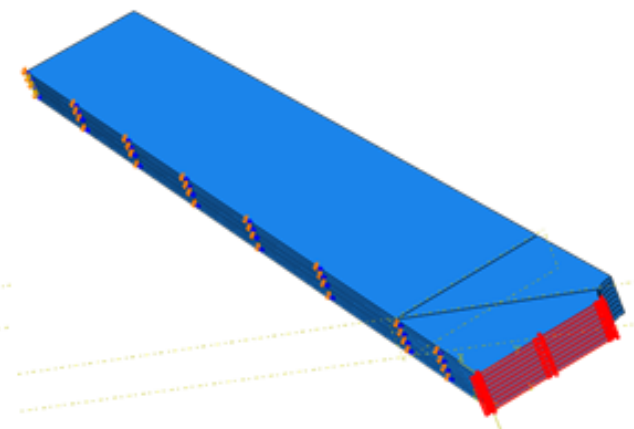

Y-symmetric

Figure 6.1: Asymmetric boundary conditions on $X$ and $Y$ axes of quarter sample

Material properties were detailed in Chapter 2 (a summary listed in Appendix B); where material \#1 referred to single ply engineering constants in the case of modelling composite lay-ups (cases \# 1 and 4) and material \#2 referred to isotropic homogeneous material (cases \# 2, 3, 5 and 6). Isotropic material was assumed in these models due to limitations in Abaqus, as will be detailed hereunder. 
Except for models \# 3 and 6, the model cell was partitioned in order to help define the mesh. The edges were assigned a biased seeding, Figure (6.2), resulted in a mesh composed of 95612 nodes and 87680 elements (8-noded brick linear hexahedral elements of type C3D8R) seen in Figure (6.3). Consistent units were kept for all analyses where length was measured in mm, stress in MPa and density in tonne $/ \mathrm{mm}^{3}$.

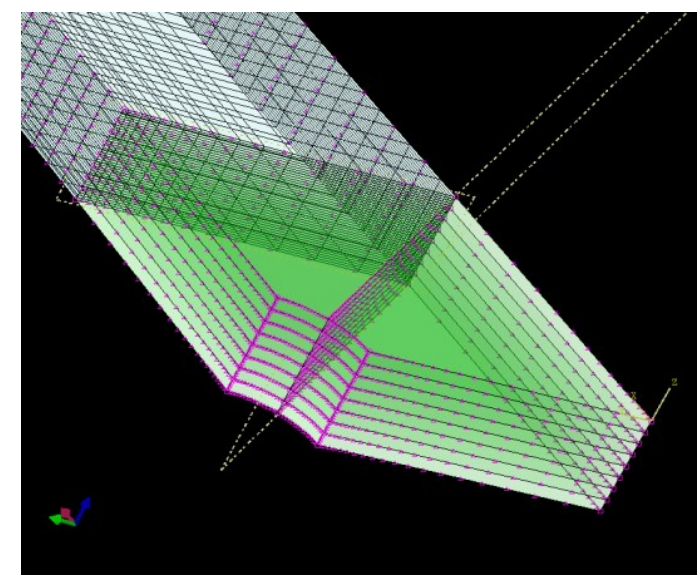

Figure 6.2: Biased seeding of 3D Abaqus model

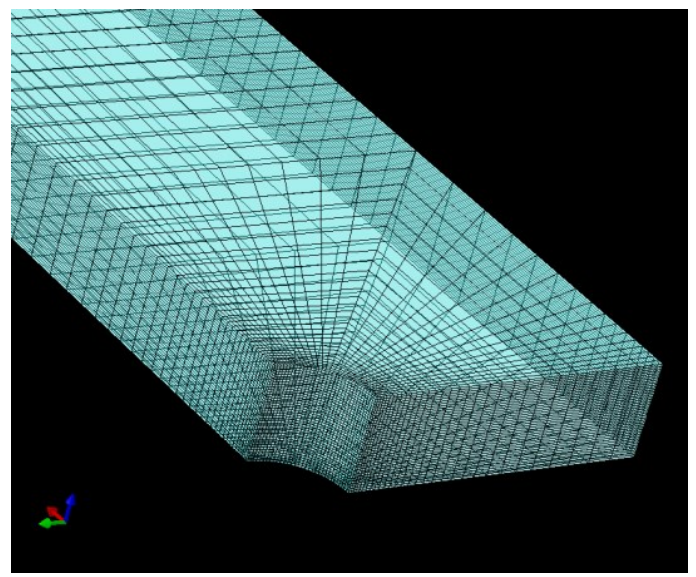

Figure 6.3: 3D Abaqus model mesh

\subsubsection{Model case 1}

This case was modelled to study the SCFs as compared to theoretical values for verifying material properties. No crack was assembled within the model, hence allowing to construct an 8-ply composite lay-up, Figure 6.4 , with a stack configuration of $[0,90,0,90] \mathrm{s}$. Ply material constants listed in Appendix B were used. A $100 \mathrm{MPa}$ tensile load was applied.

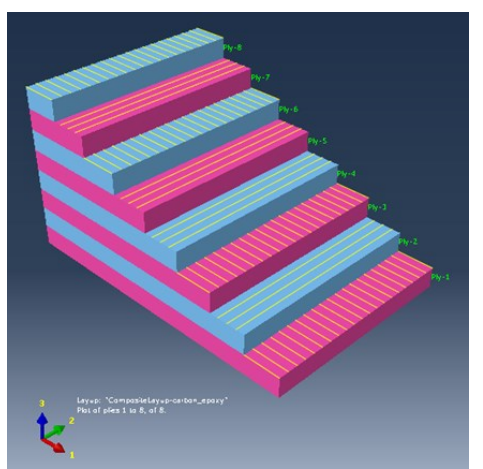

Figure 6.4: Abaqus composite lay-up

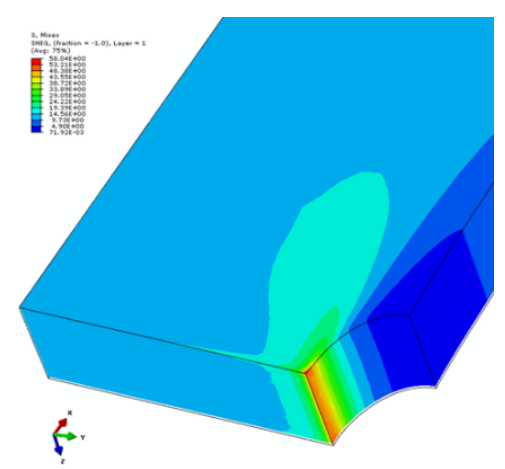

Figure 6.5: Principle stress in Case 1 
A theoretical orthotropic stress concentration factor $\boldsymbol{K}_{\boldsymbol{t}}$ for a finite width specimen can be determined from the following expression,

$$
K_{t}=\alpha . K_{\text {too }}
$$

Where $\boldsymbol{\alpha}$ and $\boldsymbol{K}_{t \infty}$ are the finite width correction factor and the stress concentration factor for an infinite orthotropic continuum, respectively. The stress concentration factor for an infinite orthotropic continuum containing a circular hole is given by,

$$
K_{t \infty}=1+\sqrt{\frac{E_{L}}{G_{L T}}-2 \cdot v_{L T}+2 \cdot \sqrt{\frac{E_{L}}{E_{T}}}}
$$

Where $\boldsymbol{E}_{\boldsymbol{L}}, \boldsymbol{E}_{\boldsymbol{T}}, \boldsymbol{v}_{\boldsymbol{L} T}$ and $\boldsymbol{G}_{\boldsymbol{L} T}$ are the longitudinal modulus, transverse modulus, Poisson's ratio, and shear modulus of the laminate, respectively.

The finite width correction factor for an isotropic material containing a circular hole is,

$$
\alpha=\frac{2+\left(1-\frac{d}{W}\right)^{3}}{3 *\left(1-\frac{d}{W}\right)}
$$

Where $\frac{d}{\boldsymbol{W}}$ is the hole diameter to specimen width ratio. Hence, theoretically $\boldsymbol{K}_{t \infty}$ is 6.88 and $\alpha$ is 1.03 resulting in $\boldsymbol{K}_{t}$ equal to 7.09 . The stress concentration factor is determined by the ratio of maximum principle stress, $\boldsymbol{\sigma}_{\max }$ to nominal stress, $\boldsymbol{\sigma}_{\boldsymbol{n} \text { om }}$,

$$
K_{t}=\frac{\sigma m a x}{\sigma n o m}
$$

The maximum principle stress, $\boldsymbol{\sigma}_{\max }$ was observed at the tip of the notch where minimum width was measured, Figure 6. 5. Segmentation showed that the maximum principle stress was concentrated at the notch mid-points of $0^{\circ}$ plies, Figure 6.6 , with a magnitude of 693.98 $\mathrm{MPa}$ whereas the nominal stress, $\boldsymbol{\sigma}_{\boldsymbol{n}}$, was $100 \mathrm{MPa}$, leading to estimate $\boldsymbol{K}_{\boldsymbol{t}}$ of 6.9398 with $2 \%$ deviation from the theoretical value. Theoretical results were compared to Abaqus 
outcome in Table 6.1. Maximum deflection was $0.5 \mathrm{~mm}$ at the loaded end. The CPU processing time was 25 minutes on a 16 GB RAM.

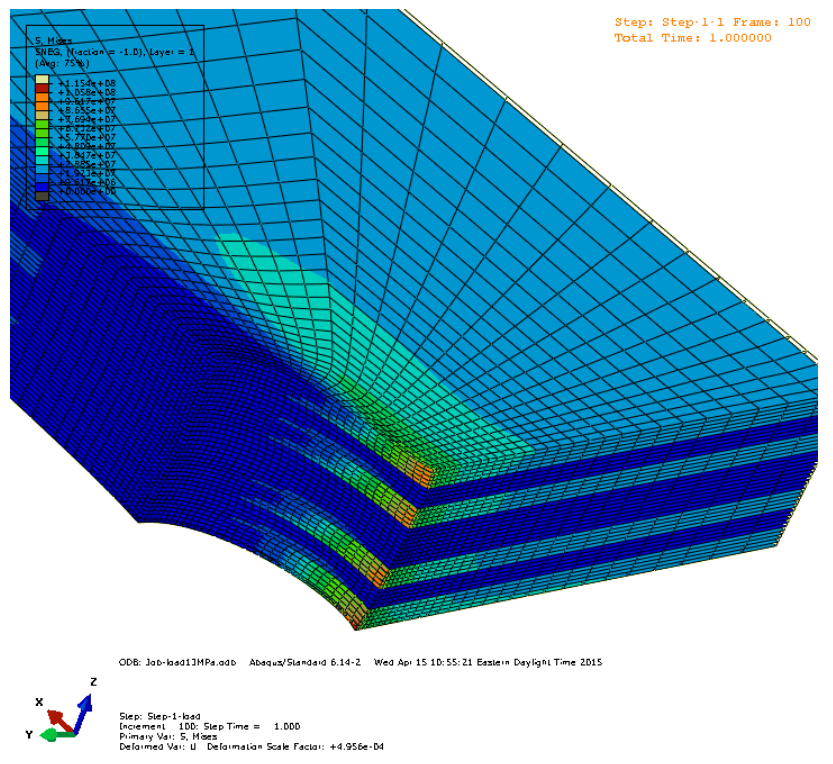

Figure 6.6: Principle stress contours of the segmented model case 1

Table 6.1: Calculation of stress concentration factor, $\boldsymbol{K}_{\boldsymbol{t}}$

\begin{tabular}{|c|c|c|c|}
\hline \multicolumn{3}{|c|}{$K_{t}$} \\
\hline \multicolumn{2}{|c|}{ Theoritical } & \multicolumn{2}{c|}{ Abaqus results } \\
\hline$K_{t \infty}$ & 6.88 & $\sigma_{\max }$ & $693.98 \mathrm{Mpa}$ \\
\hline$\alpha$ & 1.03 & $\sigma_{n o m}$ & $100.00 \mathrm{Mpa}$ \\
\hline \multicolumn{2}{|c|}{7.09} & \multicolumn{2}{|c}{6.94} \\
\hline
\end{tabular}

\subsubsection{Model case 2}

An accurate determination of the SIF is vital for reliable fatigue crack growth prediction. SIFs are dependent on load condition as well as on geometry. Hence, case 2 addresses a model with a predefined stationary crack of $2 \mathrm{~mm}$ long and $0.8 \mathrm{~mm}$ deep located at midpoint of the quarter-notch, Figure 6.7. History output data were requested for the SIF and $J$-integrals of the crack set of nodes. Data were plotted for 3 tensile load magnitudes; 100 $\mathrm{MPa}, 200 \mathrm{MPa}$ and $300 \mathrm{MPa}$. 


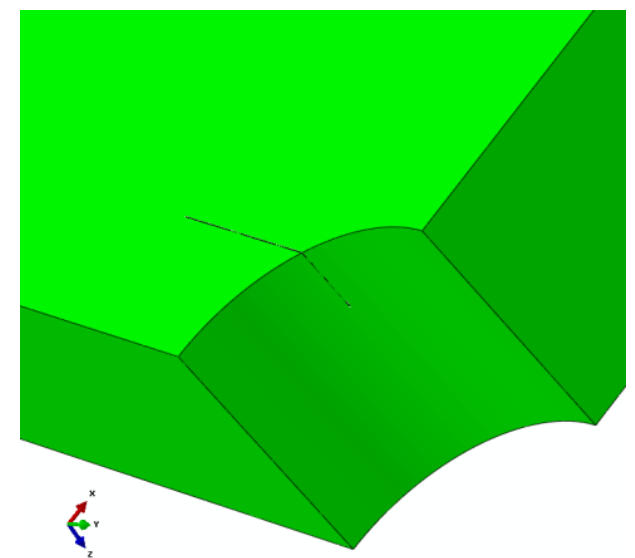

Figure 6.7: A predefined crack at mid-notch

Data of maximum energy release rates obtained from analysis in chapter 4 were input as the crack initiation criteria, where the crack was modelled using Abaqus, assuming an arbitrary, and solution-dependent crack path. The material was assumed to be homogeneous isotropic so as not to contradict with the available tool. This a major limitation in Abaqus, which does not support lay-up composite laminates for crack modelling.

Simulated maximum Von Mises stress was 1.16 GPa, Figure 6.8. Three contours of SIF, $\boldsymbol{K}_{\boldsymbol{s}}$ versus time at the crack path as obtained from the history output appear in Figure 6.9.

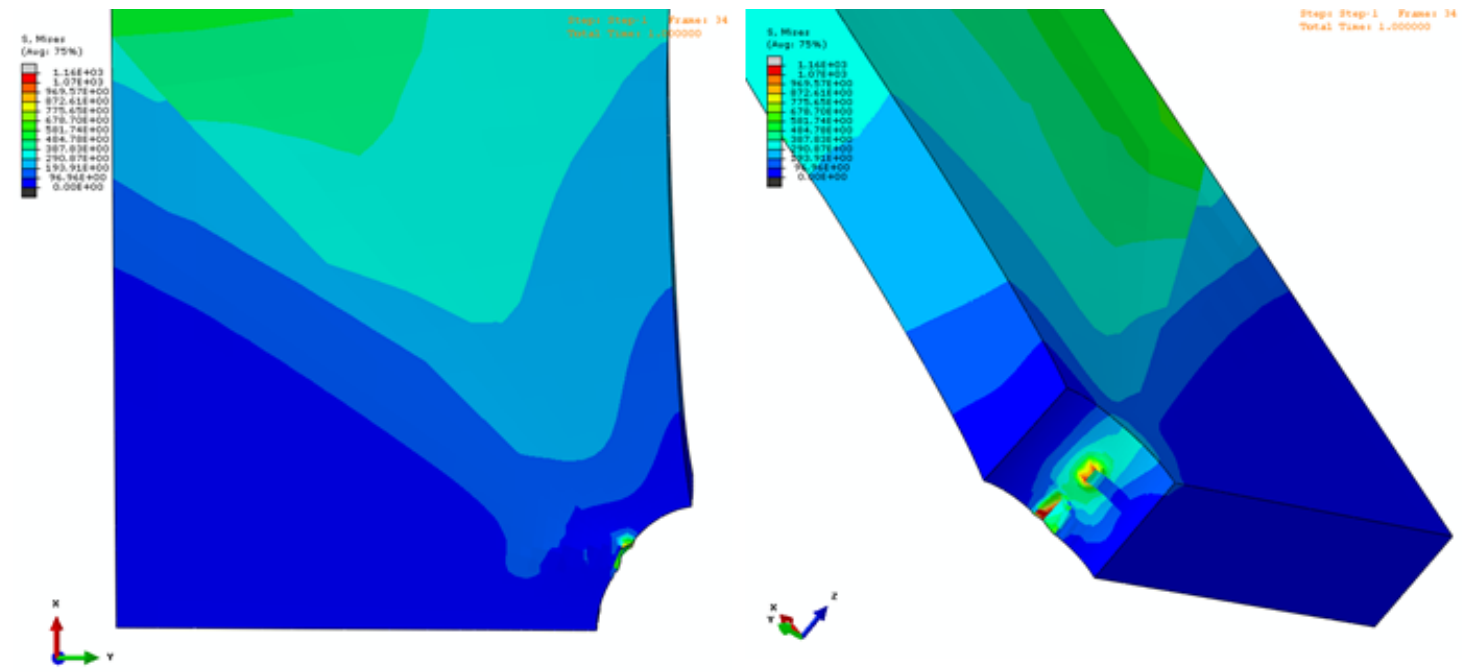

Figure 6.8: Von Mises stress contours of stationary crack 
Modelling in Abaqus
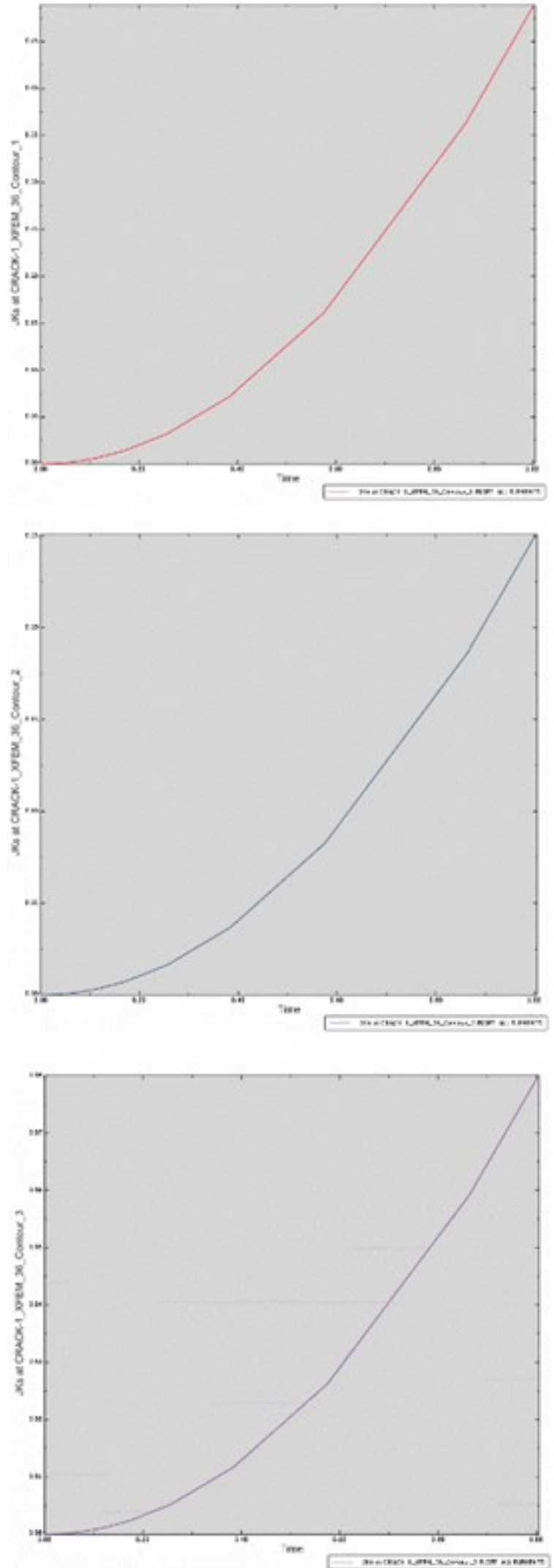

Figure 6.9: 3 Contours of $K_{s}$ versus time at the crack set of nodes 


\subsubsection{Model case 3}

A crack was studied for the same 3D model of case 4, except for substituting composite lay-ups with 8 segments and consequently assuming a homogeneous isotropic material. The crack was modelled as a separate shell part of $2 \mathrm{~mm}$ long and $0.8 \mathrm{~mm}$ deep and was assembled with the sample body at mid-point of the quarter-notch, as it appears in Figure 6.10. Another limitation in studying cracks in Abaqus is that cracks should be assembled at the surface of the part; with no possibility of modelling an inside crack.

Results of analyzing the 3-mode fracture energies, detailed in chapter 5, were used for defining damage behaviour. Maximum Von Mises stress was 1.26 GPa, Figure 6.11; close to that obtained in model case 2, despite different stress contours in both. The developed crack opening is seen in Figure 6.12.

The orthogonal level set functions, psi, $\psi$, and phi, $\varphi$, were traced and recorded, Figures 6.13 and 6.14 respectively. Where $\psi \boldsymbol{y}$ is the level set describing crack tip and $\varphi$ is the level set describing the crack surface, whereas the intersection of both sets gives the crack front.

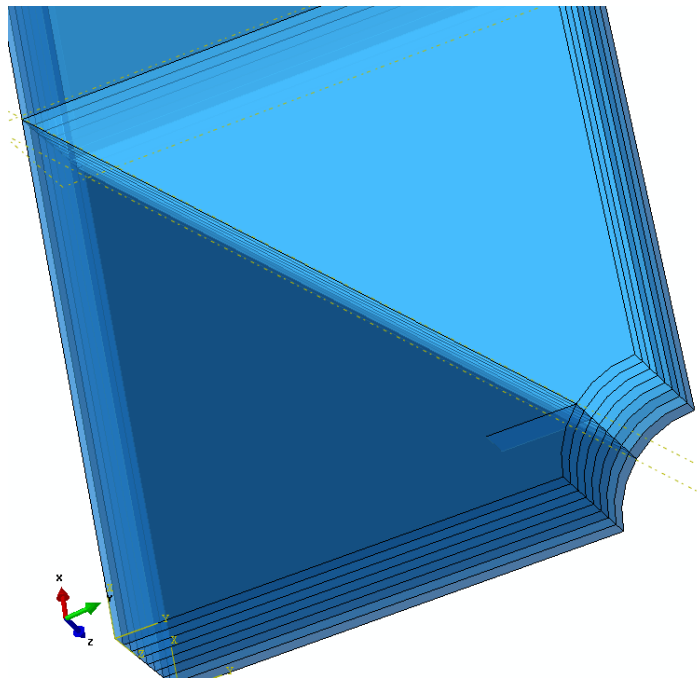

Figure 6.10: Crack location

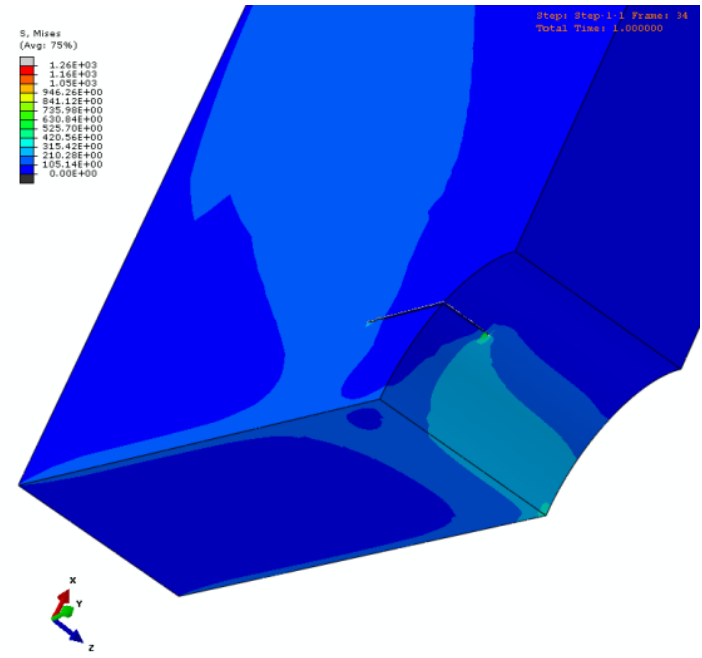

Figure 6.11: Von Mises stress contours of growing crack 

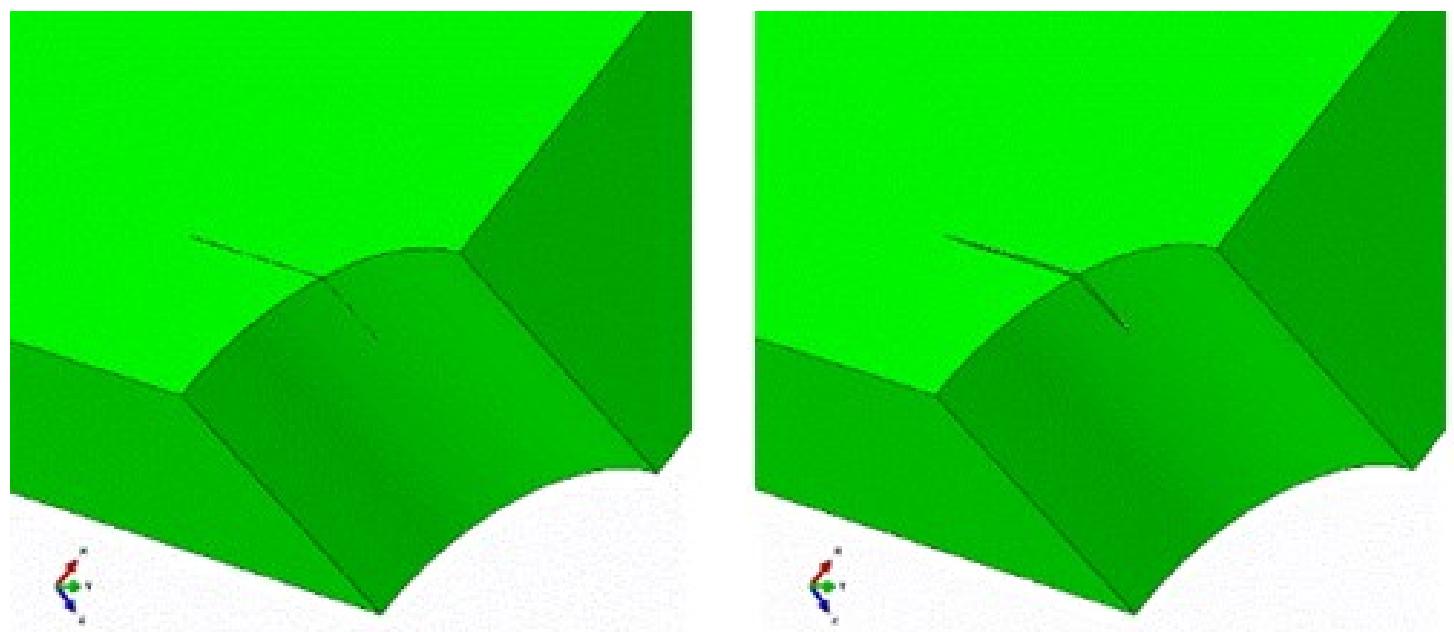

Figure 6.12: Crack before loading (left) and after loading (right)
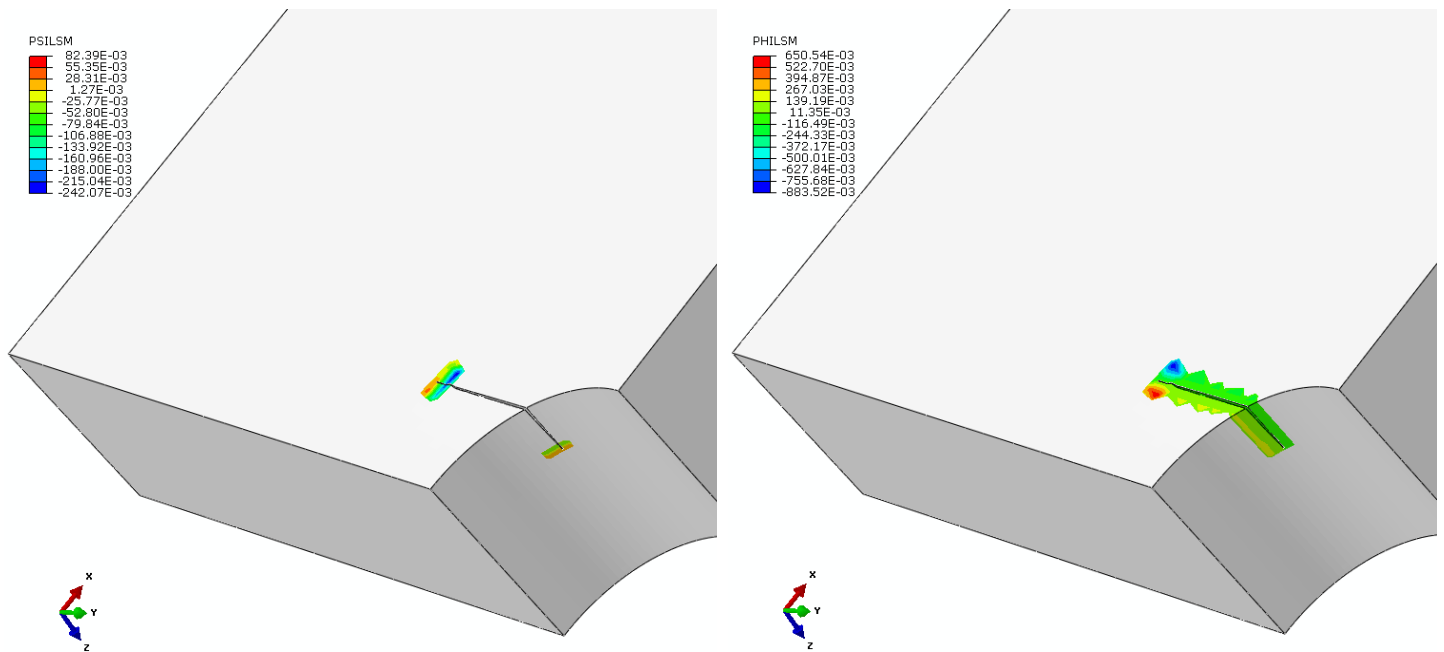

Figure 6.13: level set $\psi$ for crack

Figure 6.14: level set $\varphi$ for crack

\subsubsection{Model case 4}

Simulation in Abaqus was carried out on a definite VOI imported from microCT scans. The volume addressing part of the notch was defined over 59 successive images using CTAnalyzer software [66]. The size of the images were $913 \times 412$ pixels, and the images were 1 pixel width apart. Considering the pixel size $=12 \mu \mathrm{m}$, so the volumes of imported parts were $11 \times 4.9 \times 1.71 \mathrm{~mm}^{3}$, Figure 6.15 . 


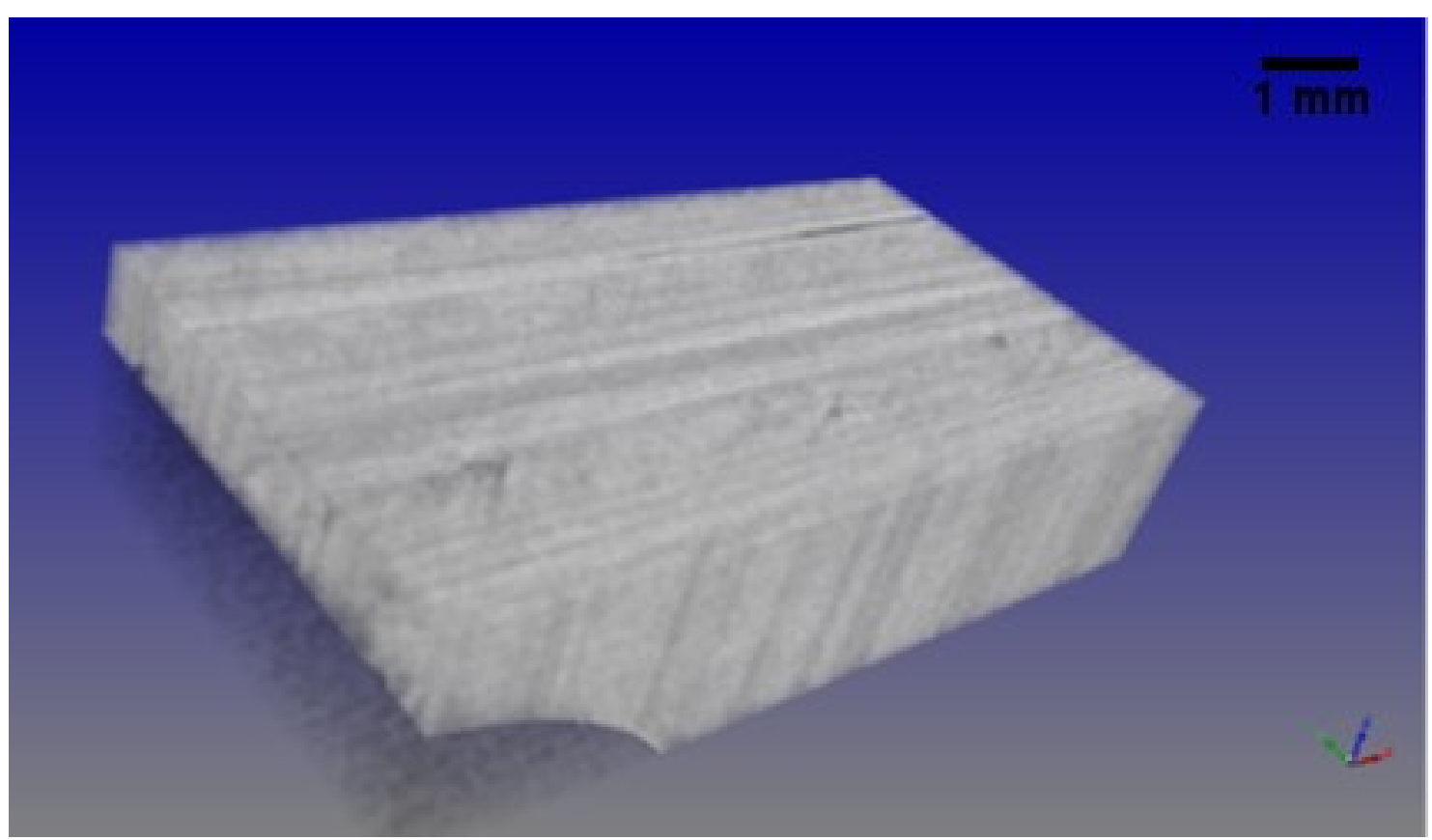

Figure 6.15: VOI of 59 images imported from microCT scans

Sets of images were imported to ScanIP software environment (Simpleware Ltd, Exeter, UK) [104] where the parts were segmented and meshed and then imported into Abaqus. The acquired sets of images were volume rendered, and their transparency was adjusted using ScanIP [104] tools. Hence, the void cracks were clearly visualized.

The procedure was repeated for all microCT scans throughout the 7 phases of fatigue life described in Chapter 4. Transparent volumes are compared in Figure 6.16 where extremely slight changes were observed, confirming the analysis detailed in Chapter 4.

One horizontal crack followed an unexpected pattern from closing after $200 \mathrm{~K}$ cycles, keeping a constant geometry for successive fatigue phases, then expanding after $800 \mathrm{~K}$ cycles and finally reduced in size. 


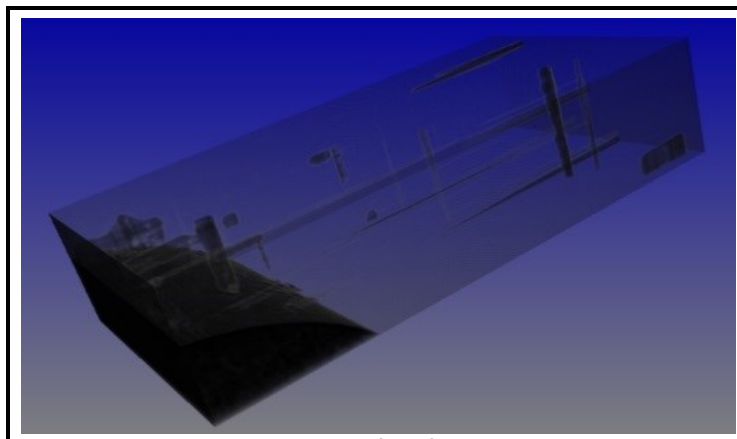

( a )

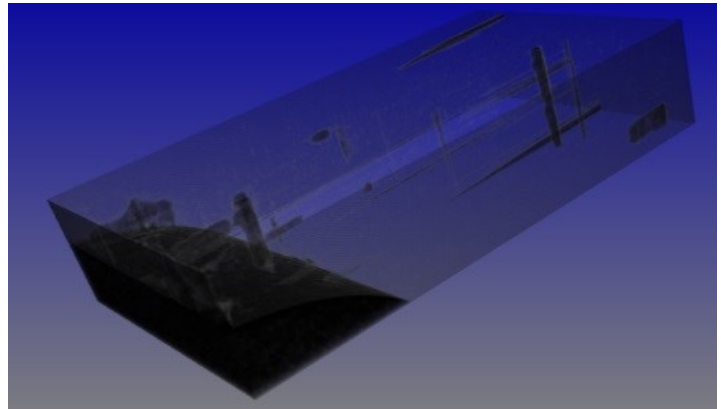

(c)

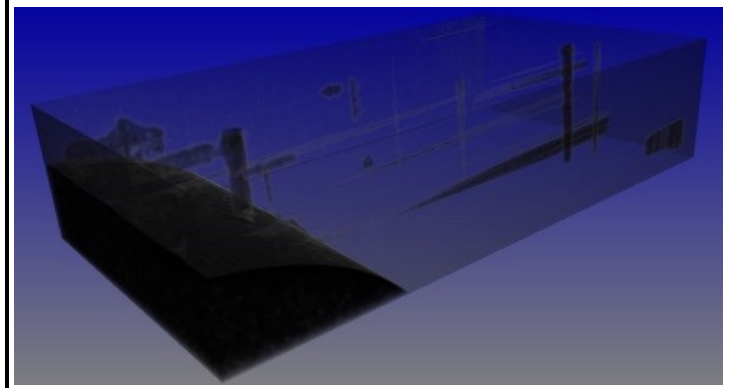

( e )

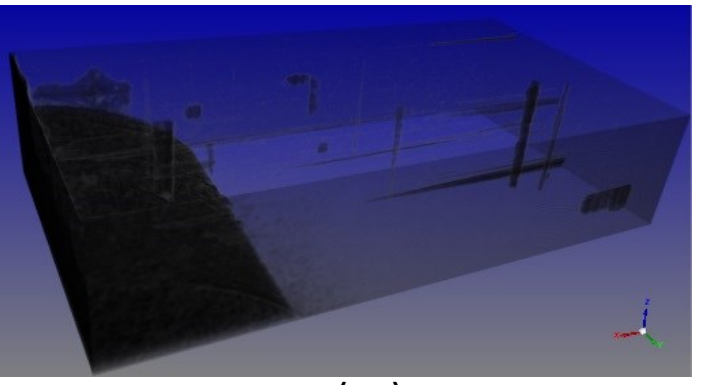

( g )

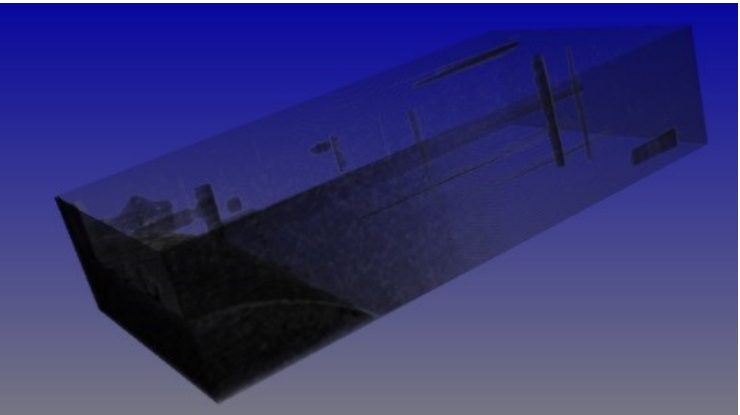

(b)

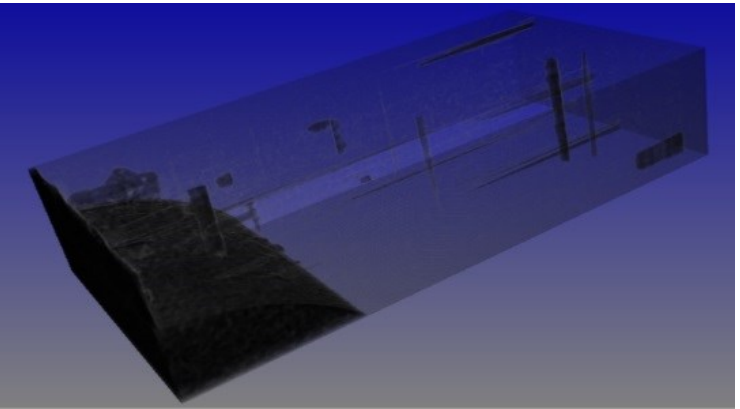

(d)

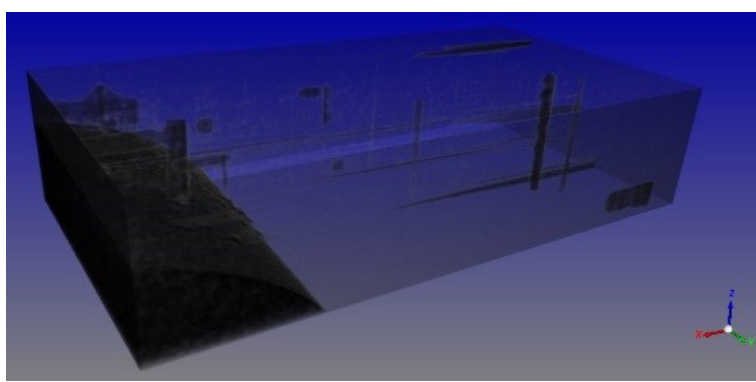

( $f$ )

Figure 6.16: Rendered volumes of successive phases of fatigue life

Segmentation process in ScanIP [104] was based on a manually selected threshold value of 90 relative to greyscale values, which resulted in 2 masks, i.e. matrix and voids. Below that value the volume corresponded to the voids, i.e. cracks mask, Figure 6.17, and above 
it the volume corresponded to the matrix mask, Figure 6.18. The cracks mask was set as a non-exportable mask, but the nodes constructing these voids were exported into Abaqus in a distinctive set used for defining the crack location of the assembly.

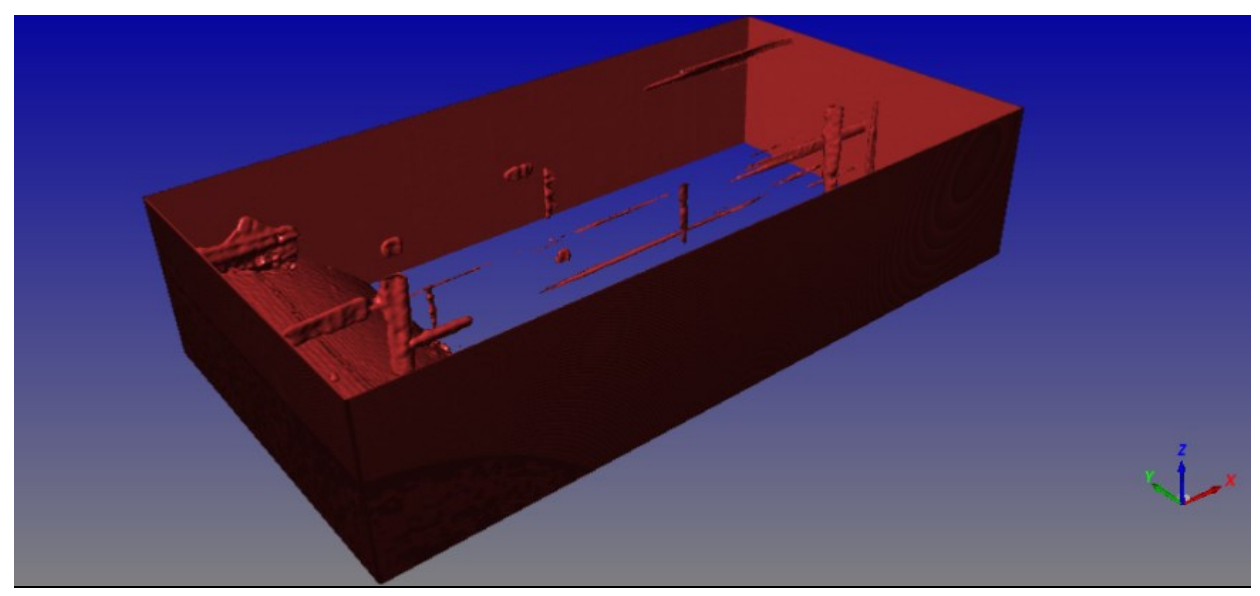

Figure 6.17: Cracks mask of the VOI imported from microCT

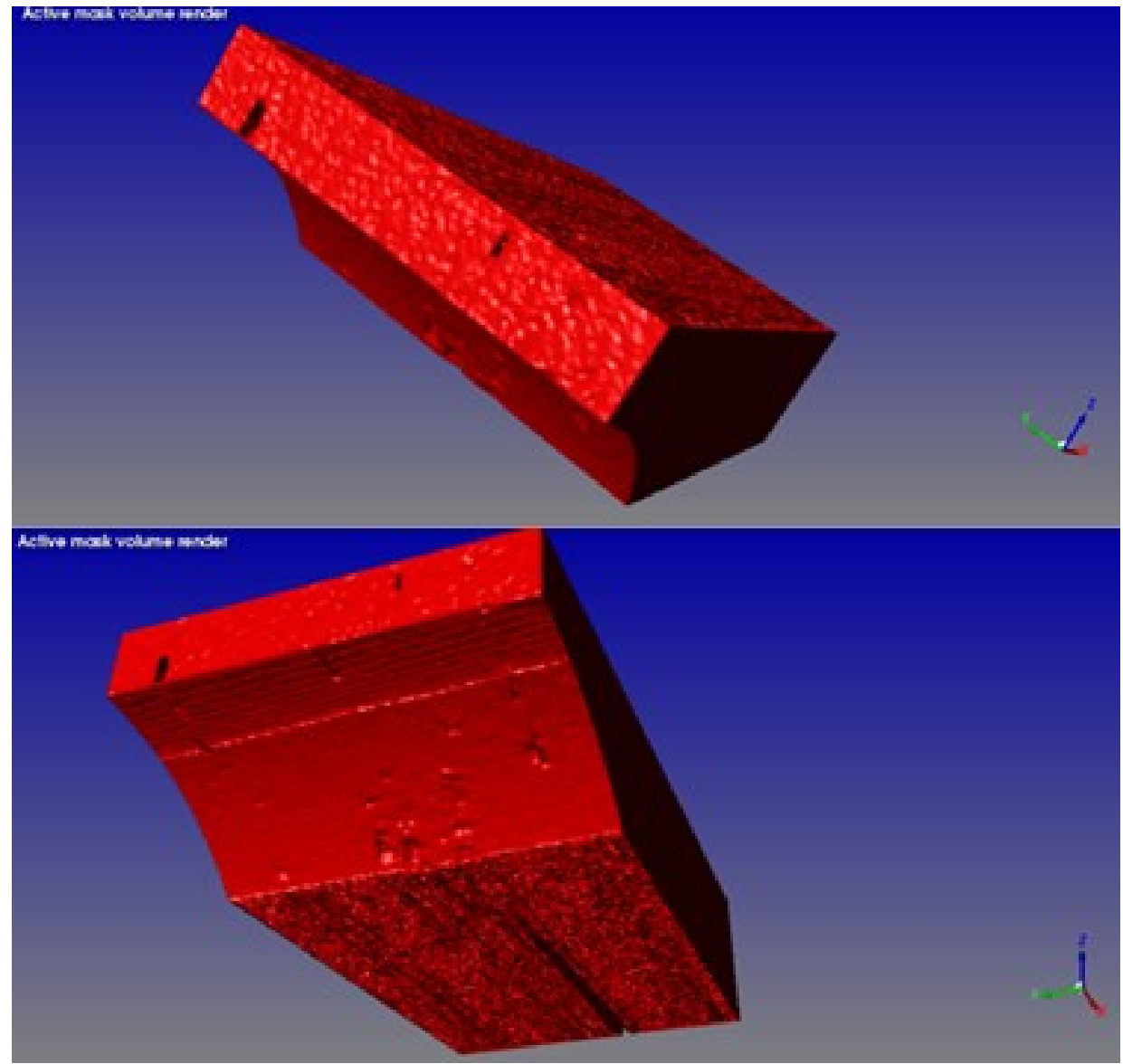

Figure 6.18: Matrix mask of the VOI imported from microCT 
To save computational efficiency, meshing was performed in a simple form of quadratic tetrahedral C3D10 (10-node) type, resulting in 513,405 elements, Figure 6.19.

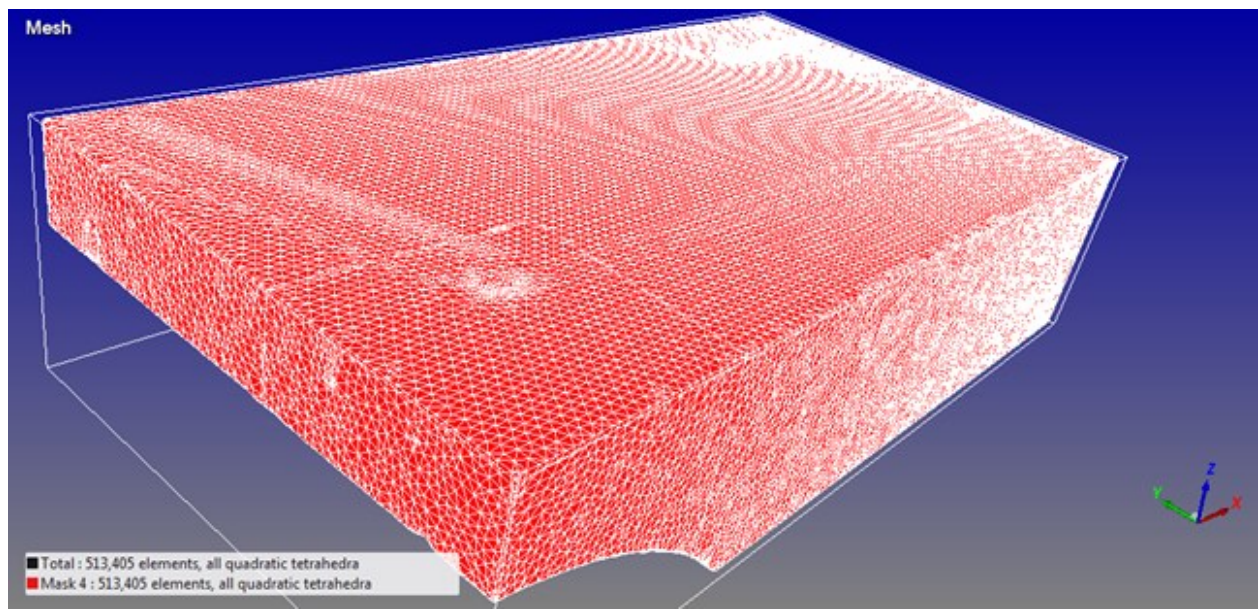

Figure 6.19: Matrix quadratic tetrahedral mesh of VOI

Cracks were spotted using transparent volume rendered images, Figure 6.16. Virtual cuts were plotted using tools of ScanIP software [104] to slice the meshed VOI where cracks were spotted, Figure 6.20, and the crack dimensions were clearly identified.

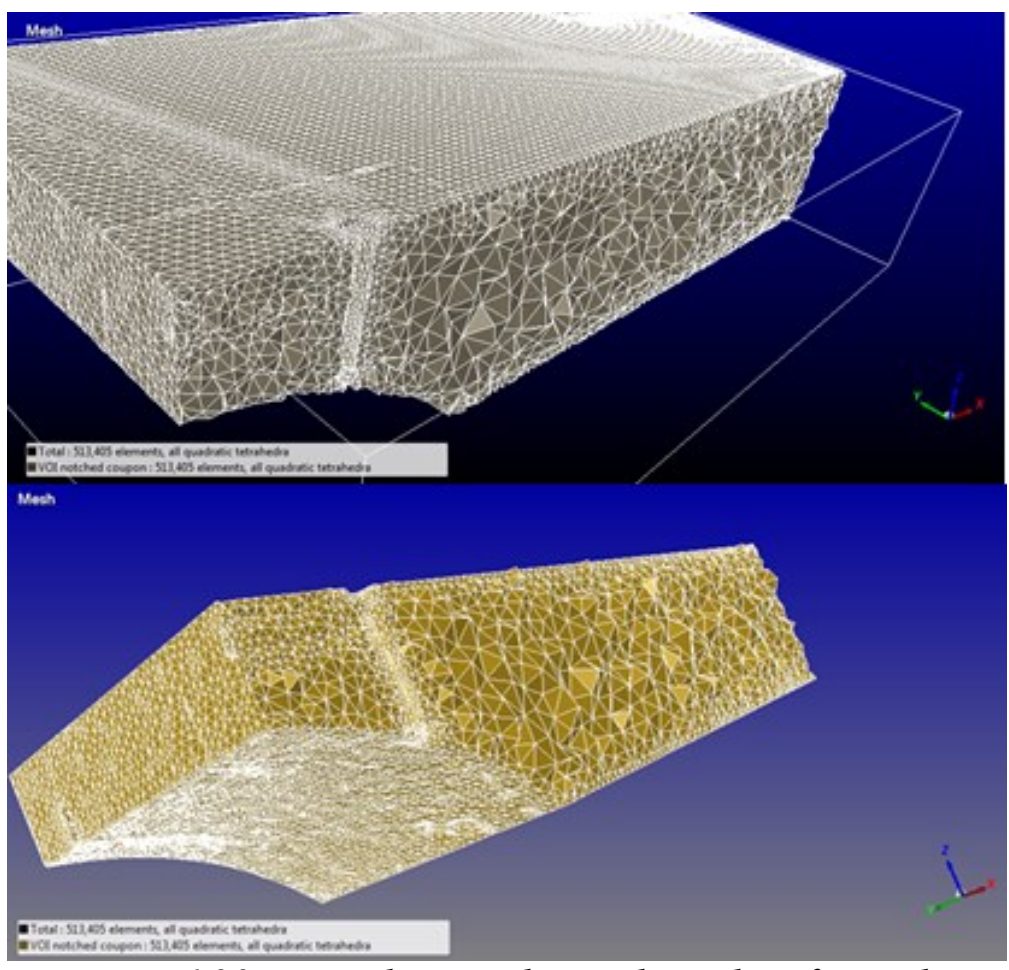

Figure 6.20: Virtual cut in the mesh to identify cracks 
Following the rendered volume visual analysis, vertical, horizontal, and an L-shaped cavities were traced in the solid meshes, Figure 6.21. However, tracing their geometries throughout the fatigue life phases did not confirm acute sensible changes throughout the fatigue life intervals.

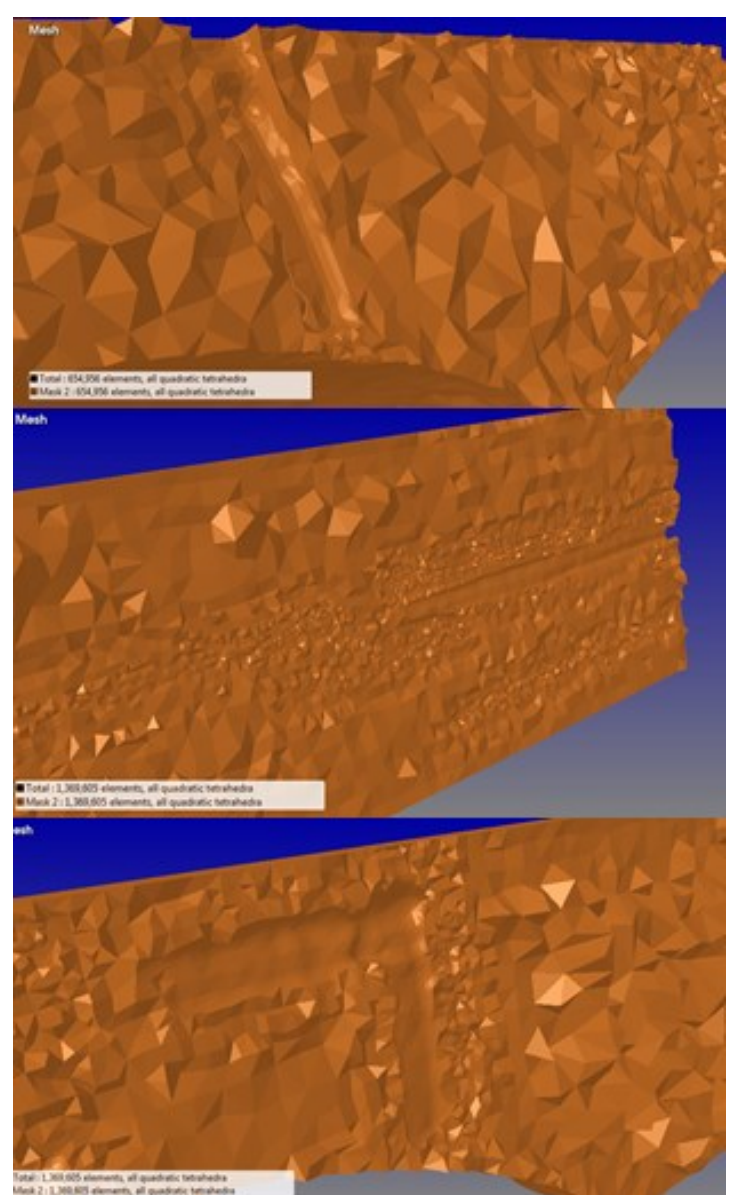

Figure 6.21: Cavities traced in virtual slices of solid mesh

- Density maps

Material properties were defined in terms of density corresponding to greyscale values obtained from microCT scans. Density mappings were manually set as 3-step, which could have been as many as 10-step, but the greyscale histogram showed only 3 levels of greyscale values which reflects the intensity of x-ray attenuation being relative to densities. Datum density of the 0-greyscale was manually input $=1.225 \mathrm{E}-12$ (air density- mimicked 
voids) whereas input density relative to the highest greyscale 255 , was manually input 1.5058E-9 (mimicked material density- Appendix B). Density units were in tons $/ \mathrm{mm}^{2}$ to be consisted with Abaqus units. Models imported into Abaqus included 3 types of materials assigned to their relative 3 sections. Density values of the 3 sections over the 7 fatigue phases are listed in Table 6.2. A graphical representation of the change in density is seen in Figure 6.22, showing an almost steady value over fatigue life for the 3 sections. Density contours were plotted on virtual slices of meshed volumes, Figures 6.23, showing a prevailing medium material density and air density concentrated in the crack cavities.

Table 6.2: Density values for fatigue phases

\begin{tabular}{|c|c|c|c|}
\hline $\begin{array}{c}\text { No of cycles } \\
\text { (thousands) }\end{array}$ & material 1 & material 2 & material 3 \\
\hline 0 & $7.53512 \mathrm{E}-10$ & $1.25504 \mathrm{E}-09$ & $2.51987 \mathrm{E}-10$ \\
\hline 200 & $6.91754 \mathrm{E}-10$ & $1.01737 \mathrm{E}-09$ & $1.34299 \mathrm{E}-09$ \\
\hline 400 & $6.45555 \mathrm{E}-10$ & $1.13712 \mathrm{E}-09$ & $1.38 \mathrm{E}-09$ \\
\hline 600 & $6.51035 \mathrm{E}-10$ & $1.13947 \mathrm{E}-09$ & $1.38 \mathrm{E}-09$ \\
\hline 800 & $6.89120 \mathrm{E}-10$ & $1.01579 \mathrm{E}-09$ & $1.34246 \mathrm{E}-09$ \\
\hline 1200 & $6.94356 \mathrm{E}-10$ & $1.01893 \mathrm{E}-09$ & $1.34351 \mathrm{E}-09$ \\
\hline 1800 & $6.91754 \mathrm{E}-10$ & $1.01737 \mathrm{E}-09$ & $1.34299 \mathrm{E}-09$ \\
\hline
\end{tabular}

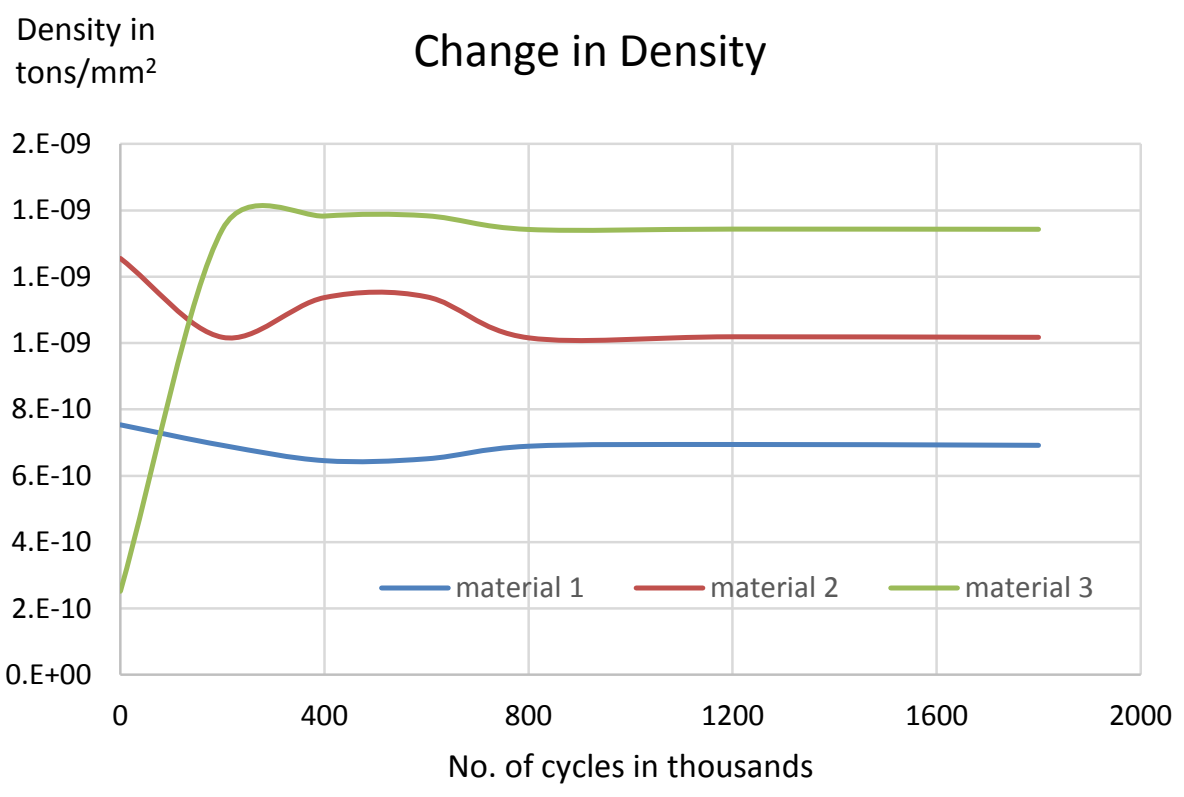

Figure 6.22: Plots of density over fatigue life for 3 material sections 


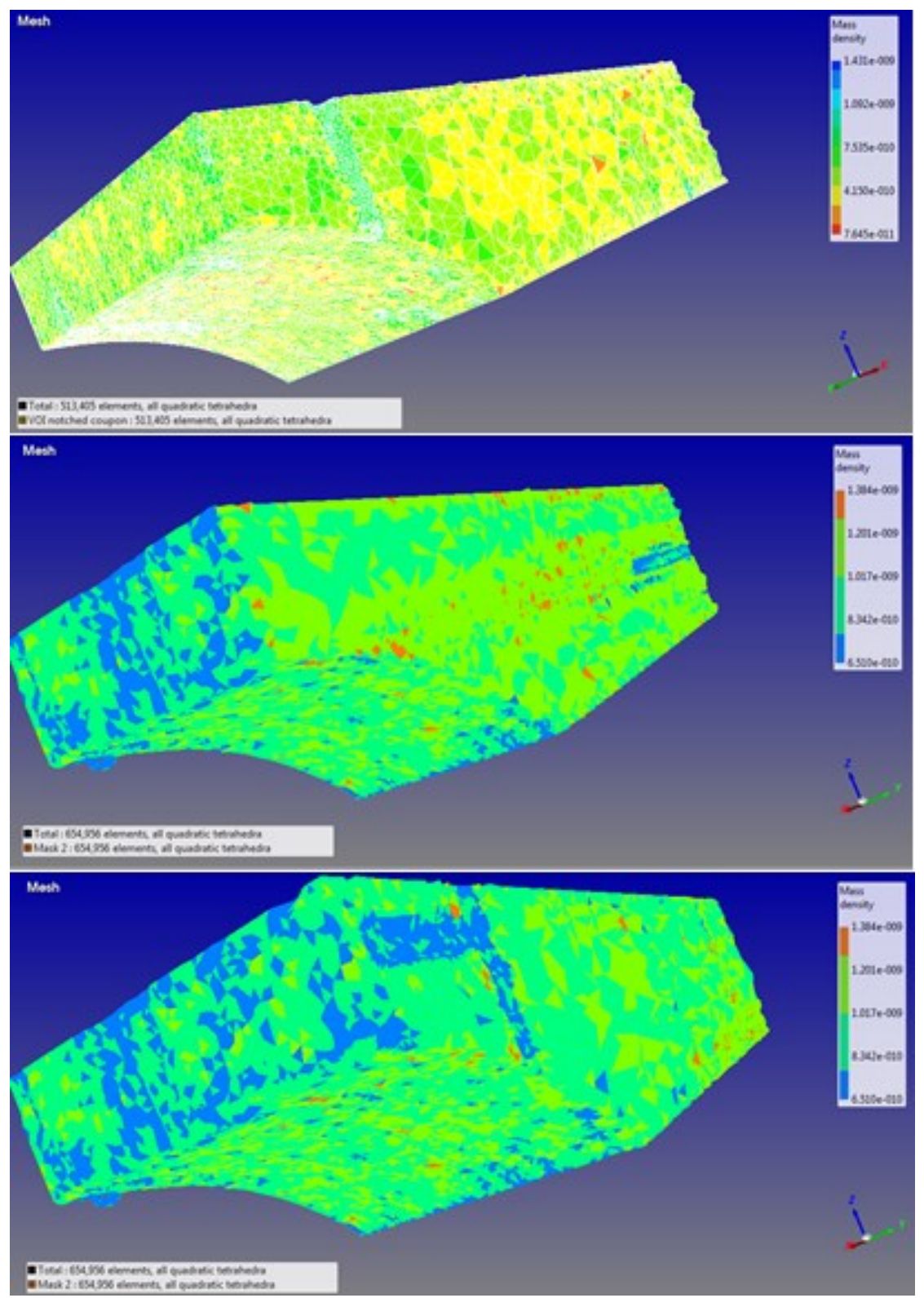

Figure 6.23: Plots of density contours on virtual slices of meshed volumes

- Importing into Abaqus

Orphan mesh of the VOI of pristine microCT scans was imported into Abaqus; having no features except material sections with their assigned properties as well as sets of elements, nodes and surfaces with no associated geometry. Voids were not included in the mesh, since the void mask was set as a non-exportable mask in ScanIP [104], Figure 6.24. However, nodes constructing these voids were imported in a separate set. 

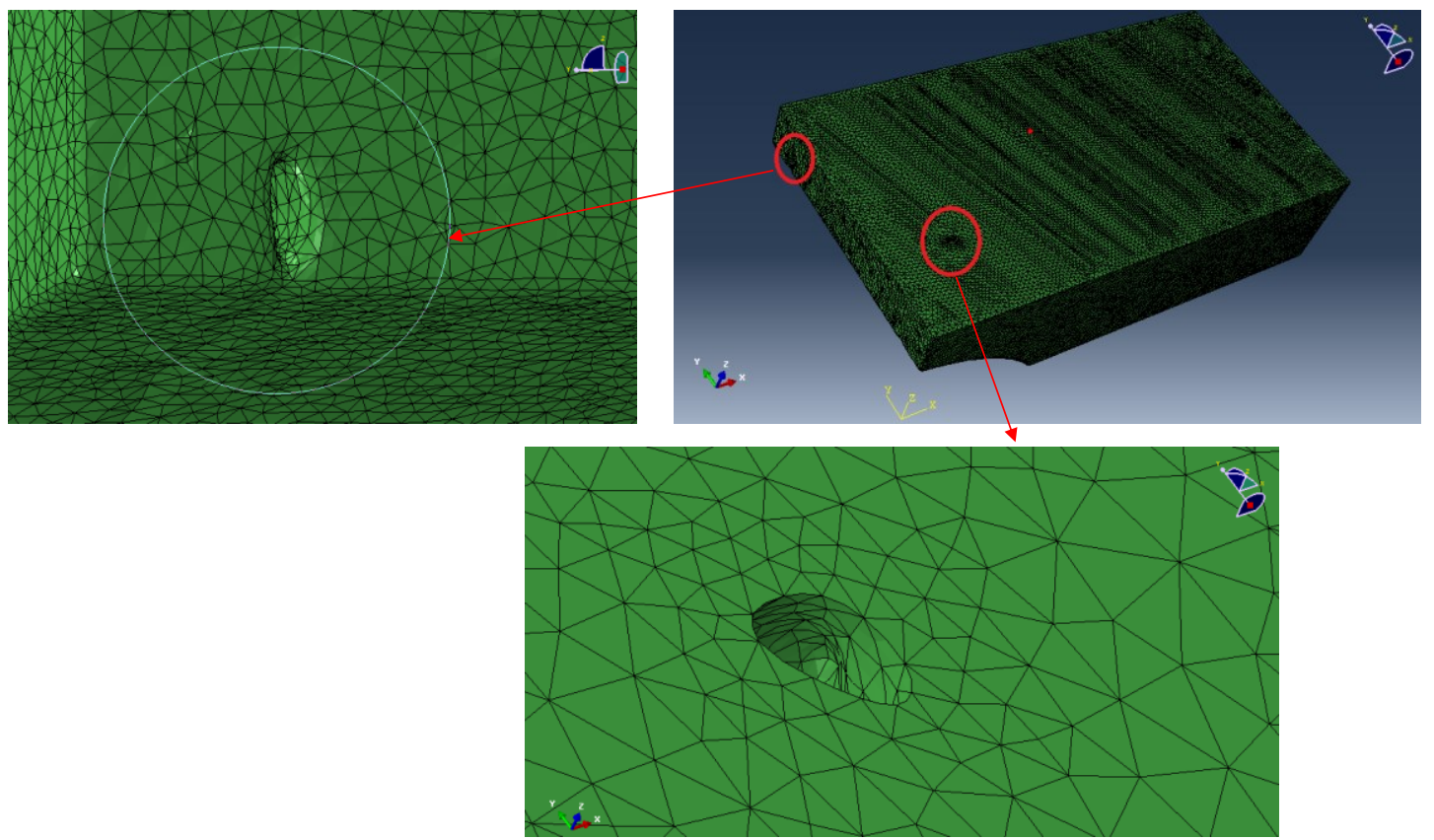

Figure 6.24: Orphan mesh of VOI imported into Abaqus excluding voids mask

A crack was created and assembled with the part, where nodes constructing the void cavities were selected as cracks locations, Figure 6.25 (a). Sets of extreme boundaries of the part in $\mathrm{X}, \mathrm{Y}$ and $\mathrm{Z}$ directions were imported from ScanIP [104], so as to use them to define boundary conditions for the orphan mesh. Minimum $\mathrm{Z}$ boundary was assigned pinned boundary condition whereas maximum $\mathrm{X}$ boundary was assigned asymmetric condition. Tensile load of $100 \mathrm{MPa}$ was applied on the minimum Z boundary, Figure 6.25 (b).

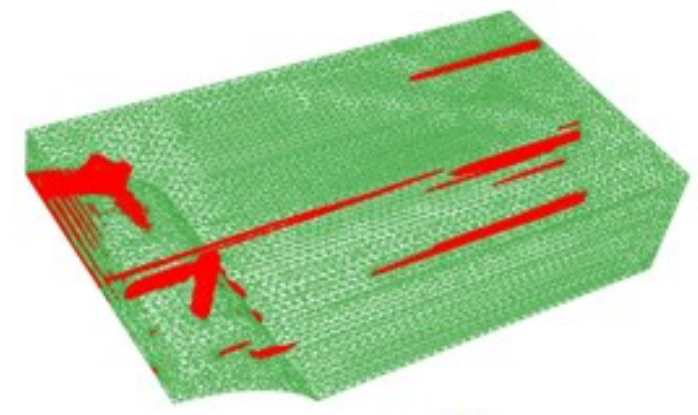

(a)

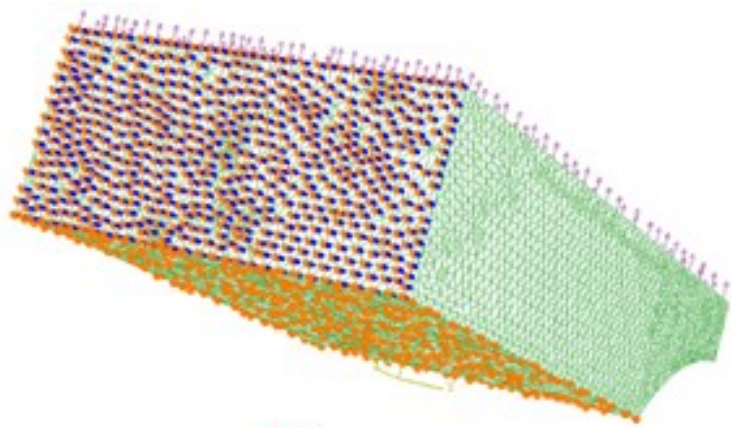

(b)

Figure 6.25: (a) Cracks locations and (b) boundary conditions on orphan mesh 
Simulation showed maximum principle stress of magnitude $15.4 \mathrm{MPa}$ at the line border of the notch,whereas maximum Von Mises stress was $11.13 \mathrm{MPa}$, Figure 6.26. Change in length ranged from $0.1 \mathrm{~mm}$ at the bottom edge to a deflection of $1.25 \mathrm{~mm}$ at the top border which is $1.7 \mathrm{~mm}$ apart according to the dimensions of the VOI investigated, Figure 6.27 .

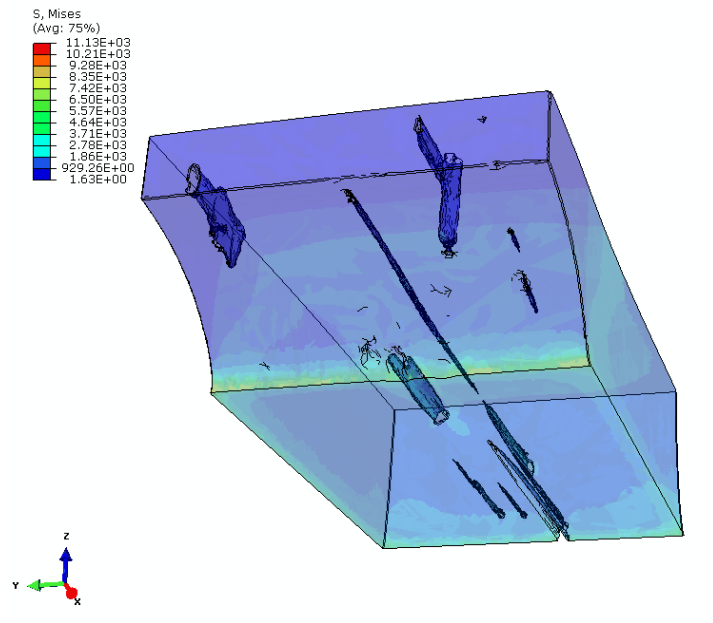

Figure 6.26: Von Mises stresses, model 6

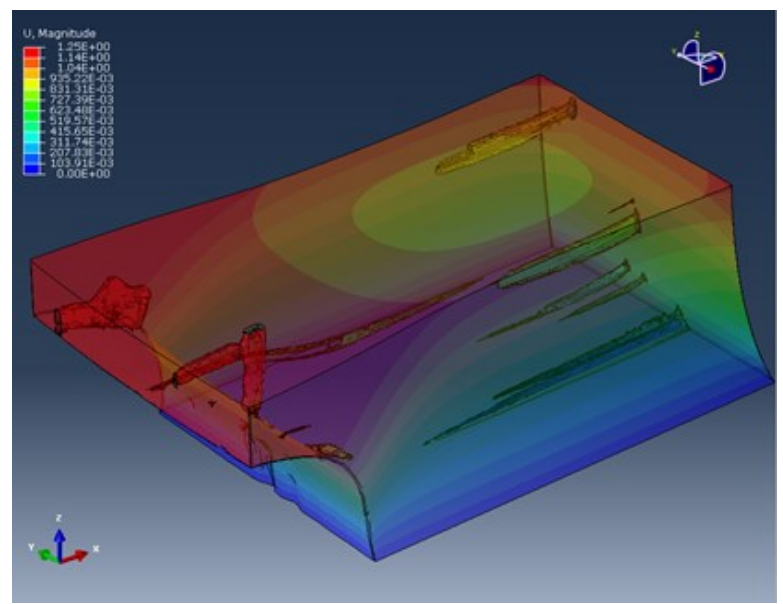

Figure 6.27: Deflection contours, model 6

Cross-sections of the deformed model, Figure 6.28, show skewness of the cracks resulting in change in their length. The change in length over fatigue intervals agreed with the experimental values, confirming the hypothesis of extremely small, almost negligible deflection; that maybe attributed the overall deflection of the part rather than acute stress causing distinctive crack propagation.
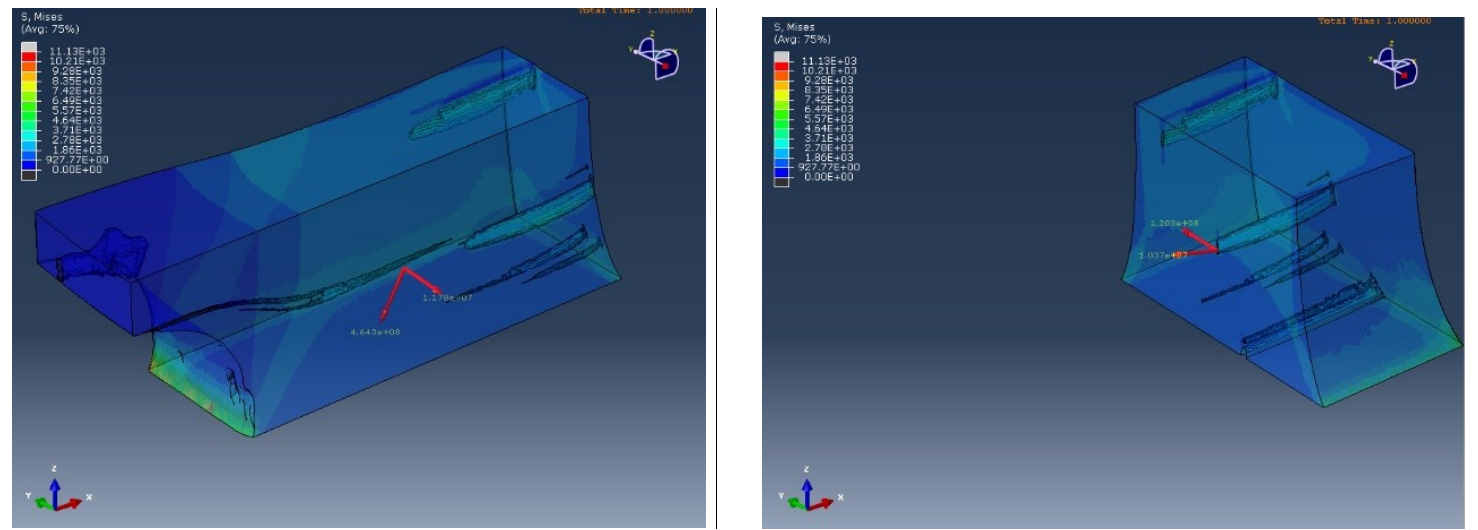

Figure 6.28: Cross-section of deformed model 6 across $Y$-axis (left) and X-axis (right) 
A smaller partition of the VOI was processed, showing plots of deflection contours. Regular increase in deflection magnitude was predicted by Abaqus, Figure 6.29, with a maximum deflection reaching $1.25 \mathrm{~mm}$. Virtual cross-section across one void showed a relative change in size.
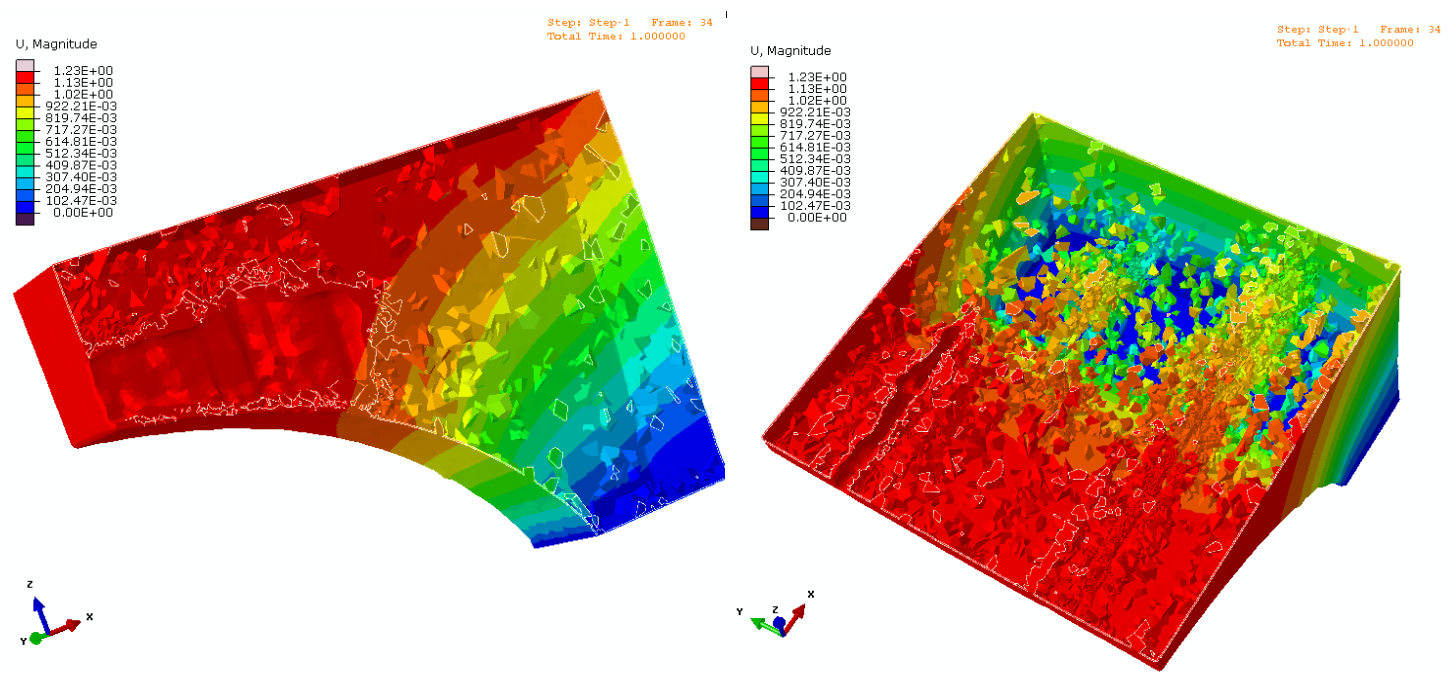

Figure 6.29: Plots of deflection contours, partition of model 6

\subsection{Summary of FEA Models}

Table 6.3: A summary of models inputs

\begin{tabular}{|c|c|c|c|c|c|c|c|}
\hline \multirow{2}{*}{ Model \# } & \multirow{2}{*}{ Objective } & \multirow{2}{*}{ Description } & \multicolumn{5}{|c|}{ Material properties } \\
\hline & & & Elastic (type) & Damage law & Interaction properties & Damage criteria & $\begin{array}{c}\text { Damge evolution } \\
\text { (energy) }\end{array}$ \\
\hline 1 & $\begin{array}{l}\text { Verify material } \\
\text { properties/ SCF }\end{array}$ & $\begin{array}{c}\text { 3D composite lay-up / } \\
\text { no crack/ Traction- } \\
\text { separation laws }\end{array}$ & $\begin{array}{c}\text { Engineering } \\
\text { constants - } \\
\text { Material \# } 1 \\
\end{array}$ & $\begin{array}{c}\text { Traction - } \\
\text { Separation } \\
\text { laws }\end{array}$ & $\mathrm{N} / \mathrm{A}$ & MaxPs $=500$ & $\begin{array}{l}\text { NMFE }=0.1620 \\
\text { SMFE1 }=0.1934 \\
\text { SMFE2 }=0.1826 \\
\end{array}$ \\
\hline 2 & SIF extraction & $\begin{array}{c}\text { Isotropic solid 3D } \\
\text { partioned in } 8 \text { layers / } \\
\text { side crack at the notch }\end{array}$ & $\begin{array}{c}\text { Isotropic } \\
\text { homogeneous - } \\
\text { Material \#2 } \\
\end{array}$ & $\begin{array}{c}\text { Traction - } \\
\text { Separation } \\
\text { laws }\end{array}$ & $\mathrm{N} / \mathrm{A}$ & MaxPs $=500$ & $\begin{array}{l}\text { NMFE }=0.1620 \\
\text { SMFE1 }=0.1934 \\
\text { SMFE2 }=0.1826 \\
\end{array}$ \\
\hline 3 & $\begin{array}{l}\text { Study crack } \\
\text { growth }\end{array}$ & $\begin{array}{c}\text { Isotropic solid 3D } \\
\text { partioned in } 8 \text { layers / } \\
\text { side crack at the notch }\end{array}$ & $\begin{array}{c}\text { Isotropic } \\
\text { homogeneous - } \\
\text { Material \#2 } \\
\end{array}$ & $\begin{array}{c}\text { Traction - } \\
\text { Separation } \\
\text { laws }\end{array}$ & $\mathrm{N} / \mathrm{A}$ & MaxPs $=500$ & $\begin{array}{l}\text { NMFE }=0.1620 \\
\text { SMFE1 }=0.1934 \\
\text { SMFE2 }=0.1826\end{array}$ \\
\hline 4 & $\begin{array}{l}\text { Validation of } \\
\text { MicroCT scans }\end{array}$ & $\begin{array}{l}\text { VOI imported from } \\
\text { microCT scans }\end{array}$ & $\begin{array}{c}\text { Isotropic } \\
\text { homogeneous - } \\
\text { Material \#2 }\end{array}$ & $\begin{array}{l}\text { Traction - } \\
\text { Separation } \\
\text { laws }\end{array}$ & N/A & MaxPs $=500$ & $\begin{array}{l}\text { NMFE }=0.1621 \\
\text { SMFE1 }=0.1935 \\
\text { SMFE2 }=0.1827\end{array}$ \\
\hline
\end{tabular}


Table 6.4: A summary of models procedures

\begin{tabular}{|c|c|c|c|c|c|c|c|c|}
\hline \multirow{2}{*}{ Model \# } & \multirow{2}{*}{ Objective } & \multirow{2}{*}{ Load } & \multicolumn{5}{|c|}{ Crack properties } & \multirow{2}{*}{$\begin{array}{c}\text { CPU time } \\
\text { (min) }\end{array}$} \\
\hline & & & $\begin{array}{c}\text { Width } X- \\
\text { axis }\end{array}$ & $\begin{array}{l}\text { Length } \\
\text { Y-axix }\end{array}$ & $\begin{array}{l}\text { Depth Z- } \\
\text { axix }\end{array}$ & Location & Simulation method & \\
\hline 1 & $\begin{array}{l}\text { Verify material } \\
\text { properties/SCF }\end{array}$ & $-100 \mathrm{MPa}$ (tensile) & N/A & N/A & N/A & N/A & N/A & 25 \\
\hline 2 & SIF extraction & $\begin{array}{l}-100 \mathrm{MPa} \text { (tensile) } \\
-200 \mathrm{MPa} \text { (tensile) } \\
-300 \mathrm{MPa} \text { (tensile) }\end{array}$ & 0 & 2 & 0.8 & mid-point of notch & XFEM - Stationary & 6.3 \\
\hline 3 & $\begin{array}{l}\text { Study crack } \\
\text { growth }\end{array}$ & $-100 \mathrm{MPa}$ (tensile) & 0 & 2 & 0.8 & mid-point of notch & XFEM-Growing & 24.6 \\
\hline 4 & $\begin{array}{l}\text { Validation of } \\
\text { MicroCT scans }\end{array}$ & $-100 \mathrm{MPa}$ (tensile) & $\mathrm{N} / \mathrm{A}$ & N/A & N/A & node set of voids mask & XFEM-Growing & 30 \\
\hline
\end{tabular}

\subsection{Conclusion}

Limitations of modelling tensile and fatigue behaviour in laminated carbon fibre reinforced composites in Abaqus commercial FEA software can be highlighted in these points;

- Studying cracks in Abaqus limits the location of cracks for they should be assembled at the surface of the part; no possibility of assuming an inside crack.

- Cracks are precluded from being assembled with composite lay-ups, so material has to be assumed isotropic.

- A complete high cycle fatigue modelling is not feasible in Abaqus.

- Direct cycling fatigue modelling is applicable. However, interaction parameters associated with cracks are not applicable in the direct cycling step of the model, despite being active in general static steps. 


\section{Chapter 7:}

\section{Summary}

This study considered investigating damage propagation in laminated CFRP composite at the coupon level while subjected to cyclic fatigue stress, in the presence of stress concentrators, i.e. a notch. Such composite structures, despite being more commonly used to replace conventional metal parts in aircraft bodies, are still been intensely questioned in terms of durability and expected damage scenarios.

Research design incorporated both experimental and analytical procedures in order to present a phenomenological approach leading to interpret damage behaviour caused by cyclic fatigue loading. Fatigue stresses were set at a constant amplitude loading.

Material cross-ply laminated stacking sequence of $\left[0^{\circ} / 90^{\circ} / 0^{\circ} / 90^{\circ}\right] \mathrm{s}$, configuration was selected for the simplicity of theoretically estimating its mechanical properties, compared to other forms of textile structures ranging from 2D, 2.5D and 3D dimensional scales, which require highly complex modelling methods. The material was assumed to be 
homogeneous isotropic rather than orthotropic upon modelling in FEA tools, so as to overcome difficulty in modelling orthotropic or anisotropic materials using these methods.

Experimental work was carried out using a NDT method allowing detailed quantitative analysis of the material microstructure. Ultra-high resolution X-ray microCT scanner was employed to offer novel information in this regard. The goal was achieved, however, not verified. Investigating the through-thickness void content at the micro-meso scale in the virtually sliced 3 orthogonal planes, with no destructive processes associated with the experimentation, was made available. This allowed for tracking genuine pre-existing cracks rather than artificially embedded cracks commonly imposed within experimental procedures.

Both qualitative and quantitative results were obtained from the experimental work, demonstrating that damage development from a visually descriptive perspective could be performed by using the unique feature of microCT method that allows creating a volumetric virtual model by stacking numerous captured scans. Furthermore, virtual crosssections were plotted for defining damage modes. Hence, delamination, fibre breakage and matrix cracks were traced throughout fatigue life intervals. It was observed that matrix cracks joined the pre-existing delamination defects, which then lead to fibre breakage at a later and almost last stage of damage development. The observed procedure agreed with literature and implied the lead of matrix micro-cracking, followed by grow of crack density causing delamination.

Through surveying the several available tools for segmenting the scanned images, meshing the models, visualizing the $3 \mathrm{D}$ defected areas and importing the mesh into FEA tools, 
setting a robust procedure to employ these tools in the correct mode was proposed and validated. Abaqus software had been used for simulation of damage, and was used for implementing the obtained scans for running simulations and comparing to experimental information.

The procedure involved either using CTAn [66] to provide a surface .stl mesh with no geometry incorporated for rapid implementation in Abaqus; or alternatively using DataViewer [66] for obtaining virtual slices and following a several-step implementation process. While the surface mesh can provide a concise assessment of the model, obtaining a tetrahedral/hexahedral mesh could provide more information on its constituent behaviour. Virtual slices obtained from DataViewer [66] could be either imported to the integrated ScanIP software [104] for further processing, or otherwise imported to ImageJ [102] for segmentation.

In a parallel process, virtual slices were processed using a MATLAB code which provided a 3D plot of the featured damaged matrix by applying a threshold technique. The segmented masks obtained from ImageJ [102] were processed using a MATLAB-based code, Iso2mesh [105], for meshing. However, severely acute geometries featuring surfaces of cracks were not adequately addressed using this simple code, and required a more sophisticated algorithm, which could be provided by ScanIP software [104]. Tetrahedral or hexahedral mesh were obtained and imported in .inp Abaqus format.

Contrary to literature reviews that suggested identical traction-separation response for both in-plane and out-of-plane shear modes, that assumption was verified herein. Within this 
work, differentiating between the 2 modes was made possible by analyzing the virtual slices of the microCT scans in 2 separate planes relative to the 2 shear modes.

Following the proposed procedure of in-house analysis, Dataviewer [66] was used to provide through-thickness virtual slices for each of the 3 orthogonal planes of the investigated sample at 7 phases of fatigue life. Segmentation and statistical analysis of these slices was performed by ImageJ [102] for providing LEFM information regarding the crack growth rate $d a / d n$ for each plane. Choosing to analyze the segmented images orthogonally was devised in terms of the conventional 3 modes of fracture, i.e. opening, in-plane shear and out-of-plane shear modes.

In other words, information on opening mode damage, was assumed to be relative to facial cracks (fibre breakage and matrix failure), which could be extracted from X-Z plane. Whereas delamination defect which corresponds to a sliding motion, is generally studied in both in-plane and out-of-plane shear modes. Information on the in-plane shear was extracted from virtual cross-sections of the $\mathrm{Y}-\mathrm{Z}$ plane since the plies are laid normal to this plane. Information on out-of-plane shear were extracted from virtual cross sections of the $\mathrm{X}-\mathrm{Y}$ plane due to that the plies were parallel to this plane.

The strain energy release rates in the 3 orthogonal planes relative to the damage modes, associated with the fatigue loading, were hence studied and defined. The crack growth rates were analytically studied and computed based on the experimental results investigating genuine cracks. Using this outcome, finding the maximum traction and separation of each of the 3 modes was made possible. 
Verifying the deamination parameters was conducted. Analysis of the experimental work was used to compute fracture toughness by using published LEFM laws. The resultant outcome corresponded to the area under traction-separation curve. The slope of the linear traction-separation response corresponded to the material constants. Consequently, by using the available information and implementing a fatigue degradation factor, other parameters can be mathematically computed, namely; maximum traction and maximum separation.

The investigated sample was modelled in Abaqus in six different modelling cases; for verifying the SCF of the notched sample which agreed with theoretical estimation, versus the SIF which relates to the presence of a crack rather than to the geometry of specimen. Forward to 3D model, however considering limitations in Abaqus modelling. The algorithm previously set for importing microCT scans in Abaqus was used in importing a VOI of the scanned pristine sample into FEA tool, hence analyze its density maps relative to attenuation intensity of X-ray, which reflected the void (crack) contents and locations. Simulating this model in Abaqus was analogous to successive scans. 


\section{Concluding Remarks}

- Simple laminated material is the most widely used composite structure in the aerospace industry, and is prevailed over other textile reinforcements of complex geometrical structures, despite the latter can provide better performance characteristics, but modelling their failure scenarios is still a premature area of investigation.

- XR-microCT testing method was proved to be a reliable NDT tool for qualitatively and quantitatively visualizing and monitoring damage mechanisms relative to the fatigue life in composite specimens.

- By using the $\mathrm{X}$-ray microCT software, creating successive virtual cross-sections of a 3D-object was possible, allowing to recreate a virtual volumetric model.

- Statistical analysis of geometry and location of experimentally detected cracks and probing the general trend of their development throughout fatigue life, lead to computing fracture energies relative to each of the 3 fracture modes adhering to LEFM theories.

- Calibration of delamination parameters was carried out, differentiating between inplane and out-of-plane shear fracture modes.

- Simulations created by using the available FEA on-the-shelf tools could model genuine pre-existing cracks within the interior structure of the material, but damage propagation could only be studied using methods that pertain only to delamination and defect of pre-determined path. 
Overall, the main contribution of the thesis are: 1) the use of micro-CT to identify and access damage progression during fatigue testing; 2) setting a methodology to process the data from scan for visualizing damage initiation and propagation; 3) setting a methodology to import the damage model into a FE tool to be used for crack growth analysis.

\section{Future Work}

It is recommended for future work that more samples are to be scanned at extended fatigue intervals for providing more datasets and confirm the hypothesis. An overall damage parameter that addresses the accumulation of total damage as a function of fatigue cycles can hence be modelled.

To overcome limitations generally experienced with commercial FEA tools, Abaqus in particular, subroutines will be developed to address specific cases, namely, a complete high cycle fatigue modelling, which is not currently applicable. Other FEA tools for fatigue analysis, e.g. fe-safe software, will be considered as potential alternatives.

Application of cracks in case of composite lay-up is not supported which requires further investigation. In this study, the material was designed to be cross-ply. However, a simplification was adopted by assuming that the material has homogeneous isotropic properties, an assumption that was justified from a theoretical perspective. Studying cracks using the Abaqus is required to be expanded to include arbitrary locations of cracks. In the currently available application in Abaqus, the crack is designed separately and assembled to the main part such that it initiates and propagates at the surface. Interior cracks can be studied, otherwise, using Hashin's criteria. However, Hashin's method is only used with 2D shells and need to run parallel to a subroutine to address the criteria in 3D models. 


\section{References}

[1] Byrne C (2000). Technical textiles market - an overview. In: Horrocks, AR and Anand, SC, editors. Handbook of Technical Textiles. Cambridge: Woodhead Publishing, ISBN 185573385 4: pp. 1-23.

[2] Tomblin J and Seneviratne W (2011). Determining the Fatigue Life of Composite Aircraft Structures Using Life and Load-Enhancement Factors. U.S. Department of Transportation Federal Aviation Administration.

[3] Advisory Circular No: 25.571-1D (2011). Damage Tolerance and Fatigue Evaluation of Structure. U.S. Department of Transportation Federal Aviation Administration.

[4] Advisory Circular No: 20-107B (2009). Composite Aircraft Structures. U.S. Department of Transportation Federal Aviation Administration.

[5] Hinton $\mathrm{M}$ et al. (2011). Failure criteria in fibre reinforced polymer composites: Can any of the predictive theories be trusted? In: NAFEMS World Congress; Boston, USA, May 23-26, 2011.

[6] Hashin Z (1980). Failure criteria for unidirectional fibre composites. Journal of Applied Mechanics; 47: 329-334.

[7] Soden PD, Kaddour AS and Hinton MJ (2004). Recommendations for designers and researchers resulting from the world-wide failure exercise. Composite Science and Technology; 64: 589-604.

[8] Kumar RS and Talreja R (2003). A continuum damage model for linear viscoelastic composite materials. Mechanics of Materials; 35 (3-6): pp. 463-480.

[9] Kuraishi A, Tsai SW and Liu KKS (2002). A progressive quadratic failure criterion, part B. Composite Science and Technology; 62: pp. 1683-1695.

[10] Cuntze RG (2004). The predictive capability of failure mode concept-based strength criteria for multi-directional laminates- part B. Composite Science and Technology; 64: pp. 487-516.

[11] Zinoviev PA, Lebedeva OV and Tairova LP (2002). A coupled analysis of experimental and theoretical results on the deformation and failure of composite laminates under a state of plane stress. Composites Science and Technology; 62: pp. 1711-1723.

[12] Bogetti TA, Hoppel CPR, Harik VM, Newill JF and Burns BP (2004). Predicting the nonlinear response and failure of composite laminates: correlation with experimental results. Composites Science and Technology; 64: pp. 477-485. 
[13] Puck A and Schürmann H (2002). Failure analysis of FRP laminates by means of physically based phenomenological models. Composites Science and Technology; 62: pp. 1633-1662.

[14] Gotsis PK, Chamis CC and Minnetyan L (1998). Prediction of composite laminate fracture: micromechanics and progressive fracture. Composite Science and Technology; 58: pp. 1137-1149.

[15] Talreja R and Singh CV. (2012). Damage and Failure of Composite Materials. New York; Cambridge, UK: Cambridge University Press, ISBN 9780521819428.

[16] ElAgamy $\mathrm{N}$ and Laliberté J (2015). Verification of parameters for modelling of fatigue propagation in laminated CFRP Composites. In: SAMPE conference (Society for Advanced Materials and Process Engineering), Baltimore MD, USA, May 18$21,2015$.

[17] ElAgamy N, Laliberté J and Gaidies F (2015). Quantitative analysis of fatigue cracks in laminated CFRP composites using micro-computed tomography. Journal of Composite Materials. First published online on October $1^{\text {st }}, 2015$.

DOI: 10.1177/0021998315608252.

[18] ElAgamy $\mathrm{N}$ and Laliberté J (2014). Historical development of geometrical modelling of textiles reinforcements for polymer composites: a review. Journal of Industrial Textiles. First published on October 20, 2014.

DOI: $10.1177 / 1528083714555781$.

[19] ElAgamy N, Laliberté J, Gaidies F and Goldak J. (2014). Qualitative characterization of fatigue damage propagation in laminated carbon fibre reinforced polymers by using micro-computed tomography. In: SAMPE conference (Society for Advanced Materials and Process Engineering), Seattle WA, USA, June 2-6, 2014.

[20] ElAgamy N, Martin C, Sachdeva T and Laliberté J (2014). Proposed procedure to simulate 3D multi-mode fatigue behaviour in laminated CFRP using micro-CT scans in ABAQUS. In: CAMX conference (The Composites and Advanced Materials Expo), Orlando FL, USA, October 12-16, 2014.

[21] Desplentere F (2007). Multiscale Modelling of Stochastic Effects in Mould Filling Simulations for Thermoplastic Composites. Katholieke Universiteit Leuven, PhD Thesis.

[22] Lomov SV, Verpoest I and Robitaille F (2005). Manufacturing and internal geometry of textiles. In: Long AC, editor. Design and Manufacture of Textile Composites. Cambridge: Woodhead Publishing Limited, ISBN-10: 185573744 2: pp. 1-60.

[23] Complete Textile Glossary (2001). Celanese ${ }^{\circledR}$ Acetate. 
[24] Sondhelm WS (2000). Technical fabric structures - 1.Woven fabrics. In: Horrocks AR, Anand SC, editors. Handbook of Technical Textiles. Cambridge: Woodhead Publishing, pp. 62-94.

[25] Tong L, Mouritz AP and Bannister MK (2002). 3D Fibre Reinforced Polymer Composites. Oxford: Elsevier.

[26] Bogdanovich AE and Mohamed MH (2009). Three-dimensional reinforcements for composites. SAMPE Journal; 45(6): 8-28.

[27] Badawi SS (2007). Development of the Weaving Machine and 3D Woven Spacer Fabric Structures for Lightweight Composites Materials. PhD Thesis; Technischen Universitat Dresden, Germany.

[28] Mack PE and Smith MD (2003). Advances in vacuum infusion processing using spacer fabrics as engineered reinforcing interlaminar infusion media. Verdant Technologies Inc., Published by Society for the Advancement of Material and Process Engineering.

[29] Roye A and Gries $T$ (2007). 3D Textiles for advanced cement based matrix reinforcement. Journal of Industrial Textiles; 37(2): 163-173.

[30] Carvelli V, Pazmino J, Lomov SV, Bogdanovich AE, Mungalov DD and Verpoest I (2013). Quasi-static and fatigue tensile behavior of a 3D rotary braided carbon/epoxy composite. Journal of Composite Materials; 47(25): 3195-3209.

[31] Cox BN and Flanagan G (1997). Handbook of Analytical Methods for Textile Composites. NASA contractor report 4750, March 1997.

[32] Peirce F (1937). The geometry of cloth structure. Journal Textile Institute; 28(3): 45-96.

[33] Kemp A (1958). An extension of Peirce's cloth geometry to the treatment of noncircular threads. Journal of the Textile Institute Transactions; 49(1):T44-T47.

[34] Hearle JWS and Shanahan WJ (1978). An energy method for calculation in fabric mechanics. Part I: Principles of the method. Journal of the Textile Institute; 69(4): 81-91.

[35] Hearle JWS and Shanahan WJ (1978). An energy method for calculation in fabric mechanics. Part II: Examples of the application of method to woven fabrics. Journal of the Textile Institute; 69(4): 92-100.

[36] Ishikawa T and Chou T-W (1982). Stiffness and strength behaviour of woven fabric composites. Journal of Materials Science; 17(11): 3211-3220.

[37] Tsai SW and Hahn HT (1980). Introduction to Composite Materials. Lancaster, Pennsylvania: Technomic. 
[38] Naik NK and Shembekar PS (1992). Elastic behavior of woven fabric composites: I-lamina analysis. Journal of Composite Materials; 26(15): 2196-2225.

[39] Naik NK and Shembekar PS (1992). Elastic behavior of woven fabric composites: II-laminate analysis. Journal of Composite Materials; 26(15): 2226-2246.

[40] Naik NK and Ganesh VK (1995). An analytical method for plain weave fabric composites. Composites; 26(4): 281-289.

[41] Raju IS and Wang JT (1994). Classical laminate theory for woven fabric composites. Journal of Composites Technology and Research; 16(4): 289-303.

[42] Whitcomb J, Woo K and Gundapaneni S (1994). Macro finite element for analysis of textile composites. Journal of Composite Materials; 28(7): 607-618.

[43] Naik RA (1995). Failure analysis of woven and braided fabric reinforced composites. Journal of Composite Materials; 29(17): 2334-2363.

[44] Sheng SZ and Hoa SV (2001). Three dimensional micro-mechanical modeling of woven fabric composites. Journal of Composite Materials, 35(19): 1701-1729.

[45] Hivet G and Boisse P (2005). Consistent 3D geometric model of fabric elementary cell- application to a meshing preprocessor for 3D finite element analysis. Finite Elements in Analysis and Design; 42(1): 25-49.

[46] Hewitt JA, Brown D and Clarke RB (1995). Computer modelling of woven composite materials. Composites; 26(2): 134-140.

[47] Kuhn JL and Charalambides PG (1999). Modeling of plain weave fabric composite geometry. Journal of Composite Materials; 33(3): 188-220.

[48] Rao MP, Pantiuk M and Charalambides PG (2009). Modeling the geometry of satin weave fabric composites. Journal of Composite Materials; 43(1): 19-56.

[49] Robitaille F, Long AC, Jones IA and Rudd CD (2003). Automatically generated geometric descriptions of textile and composite unit cells. Composites Part A: Applied Science and Manufacturing; 34(4): 303-312.

[50] Sherburn M (2007). Geometric and Mechanical Modeling of Textiles. PhD Thesis; University of Nottingham; England.

[51] Verpoest I and Lomov SV (2005). Virtual textile composites software Wisetex: Integration with micro-mechanical, permeability and structural analysis. Composites Science and Technology; 65(15-16): 2563-2574.

[52] Vandeurzen $\mathrm{Ph}$, Ivens $\mathrm{J}$ and Verpoest I (1996). A three-dimensional micromechanical analysis of woven-fabric composites: I. geometric analysis. Composites Science and Technology; 56(11): 1303-1315. 
[53] Vandeurzen $\mathrm{Ph}$, Ivens $\mathrm{J}$ and Verpoest I (1996). A three-dimensional micromechanical analysis of woven-fabric composites: II. Elastic analysis. Composites Science and Technology; 56(11): 1317-1327.

[54] Vandeurzen Ph, Ivens J and Verpoest I (1998). Micro-stress analysis of woven fabric composites by multilevel decomposition. Journal of Composite Materials, 32(7): 623-651.

[55] Belov EB, Lomov SV, Verpoest I, Peters T, Roose D, Parnas RS, Hoes K and Sol $\mathrm{H}$ (2004). Modelling of permeability of textile reinforcements: Lattice boltzmann method. Composites Science and Technology; 64(7-8):1069-1080.

[56] Lomov SV, Verpoest I, Peeters T, Roose D and Zako M (2003). Nesting in textile laminates: geometrical modelling of the laminate. Composites Science and Technology; 63(7): 993-100.

[57] Hallal A, Younes R and Fardoun F (2013). Review and comparative study of analytical modeling for the elastic properties of textile composites. Composites: Part $B ; 50: 22-31$.

[58] Adhesive Prepregs for Composite Manufacturers. DA 4090 | DA 4092 Epoxy Prepreg System. (Last accessed on July 7, 2015). http://prepregs.com/da-40904092-epoxy-prepreg-system/

[59] Rubio-González C, Wang J, Martinez J and Kaur H (2012). Dynamic fracture toughness of composite materials. In: Tamin, MN (Editor), Damage and fracture of composite materials and structures (pp.145-146). New York; Berlin: Springer.

[60] ASTM Standard D5766/D5766M (2011). Standard Test Method for Open-Hole Tensile Strength of Polymer Matrix Composite Laminates. ASTM International, West Conshohocken, PA. DOI: 10.1520/D5766_D5766M-11.

[61] ASTM Standard D7615 (2011). Standard Practice for Open-Hole Fatigue Response of Polymer Matrix Composite Laminates. ASTM International, West Conshohocken, PA. DOI: 10.1520/D3039 -08.

[62] eLaminate(C) 1.2. ESP Composites, LLC. (Last accessed on July 7, 2015). http://www.espcomposites.com/software/eLaminate.html

[63] De Jonge, J B et al. (1973). A Standardized Load Sequence for Flight Simulation Tests on Transport Aircraft Wing Structures. Laboratorium für Betriebsfestigkeit (LBF) and Nationaal Lucht-en Ruimtevaartlaboratorium (NLR).

[64] Lourenço, BVG (2010). Damage Tolerance Design for Wing Components Procedure Standardization. M.A.Sc. thesis, Universidade Técnica de Lisboa. 
[65] ASTM Standard E1942 (2010). Standard Guide for Evaluating Data Acquisition Systems Used in Cyclic Fatigue and Fracture Mechanics Testing. ASTM International, West Conshohocken, PA. DOI: 10.1520/E1942-98R10E01.

[66] Bruker microCT (2015). http://www.skyscan.be/home.htm. (Last accessed May 9, 2015).

[67] Hung YY, Chen YS, Ng SP, Liu L, Huang YH, Luk BL, Ip RWL, Wu CML and Chung PS (2009). Review and comparison of shearography and active thermography for nondestructive evaluation. Materials Science and Engineering $R$ : Reports; 64 (5-6): pp. 73-112.

[68] Fahr A (2013).Aeronautical Applications of Non-Destructive Testing. DEStech Publications, Inc.: U.S.A. ISBN 978-1-60595-120-1

[69] HSU D K (2008). Nondestructive Inspection of Composite Structures: Methods and Practice. In: $17^{\text {th }}$ World Conference on Nondestructive Testing. Shanghai, October 25-28. International Committee for Non-Destructive Testing. pp. 2627-2640.

[70] Meola C and Toscano C (2012). Non-Destructive Evaluation of Carbon Fibre Reinforced Polymers with Ultrasonics and Infrared Thermography: An Overview on Historical Steps and Patents. Recent Patents on Materials Science 5: pp. 48-67.

[71] Reis PNB, Ferreira JAM and Richardson MOW (2011). Fatigue Damage Characterization by NDT in Polypropylene/Glass Fibre Composites. Applied Composite Materials; 18(5): pp. 409-419.

[72] Barré S and Benzeggagh ML (1994). On the use of acoustic emission to investigate damage mechanisms in glass-fibre-reinforced polypropylene. Composites Science and Technology; 52(3): pp. 369-376.

[73] Loutas TH, Vavouliotis A, Karapappas P and Kostopoulos V (2010). Fatigue damage monitoring in carbon fibre reinforced polymers using the acoustoultrasonics technique. Polymer Composites; 31(8): pp. 1409-1417.

[74] De Angelis G, Meo M, Almond DP, Pickering SG and Angioni SL (2012). A new technique to detect defect size and depth in composite structures using digital shearography and unconstrained optimization. NDT\&E International; 45: 91-96.

[75] Cuadra J, Vanniamparambil PA, Hazeli K, Bartoli I and Kontsos A (2013). Damage quantification in polymer composites using a hybrid NDT approach. Composites Science and Technology; 83: 11-21.

[76] Tarin $\mathrm{M}$ and Rotolante $\mathrm{R}$ (2011). NDT in Composite Materials with Flash, Transient, and Lockin Thermography. MoviTHERM, Inc., FLIR Technical Series.

[77] Littleton JT. Conventional Tomography. A History of the Radiological Sciences (PDF). American Roentgen Ray Society. Retrieved 29 November 2014. 
[78] Buffiere J, Maire E, Adrien J, Masse J and Boller E (2010). In Situ Experiments with $\mathrm{X}$ - ray Tomography: an Attractive Tool for Experimental Mechanics. Experimental Mechanics; 50(3): pp. 289-305.

[79] Badel P, Vidal-Sallé E, Maire E and Boisse P (2008). Simulation and Tomography Analysis of Textile Composite Reinforcement Deformation at the Mesoscopic Scale. Composites Science and Technology; 68(12): pp. 2433-2440.

[80] Schell JSU, Renggli M, Van Lenthe GH, Müller R and Ermanni P (2006). MicroComputed Tomography Determination of Glass Fibre Reinforced Polymer MesoStructure. Composites Science and Technology; 66(13): pp. 2016-2022.

[81] Hassler U, Schloetzer S and Hanke R (2008). Computed tomography for analysis of fibre distribution in carbon fibre preforms. Insight: Non-Destructive Testing and Condition Monitoring; 50(6): pp. 312-315.

[82] Djukic L, Herszberg I, Schoeppner GA, LesBrownlow (2008). Tow visualization in woven composites using x-ray computed tomography. In: International Conference on Textile Composites Texcomp9; Newark, Delaware (USA), October 2008.

[83] Schilling PJ, Karedla, BPR, Tatiparthi, AK, Verges MA and Herrington PD (2005). $\mathrm{X}$-ray computed microtomography of internal damage in fibre reinforced polymer matrix composites. Composites Science and Technology; 65: pp. 2071-2078.

[84] Bayraktar E, Antolonovich S and Bathias C (2006). Multiscale study of fatigue behaviour of composite materials by $\chi$-rays computed tomography. International Journal of Fatigue; 28(10): pp. 1322-1333.

[85] Little JE, Yuan X and Jones MI (2012). Characterization of voids in fibre reinforced composite materials. NDT and E International; 46: pp. 122-127.

[86] Cosmi F and Bernasconi A (2013). Micro-CT investigation on fatigue damage evolution in short fibre reinforced polymers. Composites Science and Technology; 79: pp. 70-76.

[87] Wright P, Fu X, Sinclair I and Spearing SM (2008). Ultrahigh resolution computed tomography of damage in notched carbon fibre-epoxy composites. Journal of Composite Materials; 42 (19): pp. 1993-2002.

[88] Wright P, Moffat A, Sinclair I and Spearing S (2010). High resolution tomographic imaging and modelling of notch tip damage in a laminated composite. Composite Science and Technology; 70 (2010): pp. 1444-1452.

[89] Scott AE, Mavrogordato MN, Wright P, Sinclair I, Spearing SM (2012). In situ fibre fracture measurement in carbon/epoxy laminates using high resolution computed tomography. Composites Science and Technology; 71: pp.1471-1477. 
[90] Scott AE, Sinclair I, Spearing SM, Thionnet A and Bunsell AR (2012). Damage accumulation in a carbon/epoxy composite: comparison between a multiscale model and computed tomography experimental results. Composites: Part A; 43: pp. 15141522.

[91] Blassiau S (2005). Modélisation des Phénomènes Microstructuraux au sein d'un Composite Unidirectionnel Carbonelépoxy et Prédiction de durée de Vie: Contrôle et Qualification de Réservoirs Bobinés. PhD thesis, École des Mines de Paris.

[92] Avizo 3D Software, FEI (2015). http://www.fei.com/software/avizo3d/ (Accessed February 19, 2015)

[93] Corum JM, Battiste RL, Liu KC and Ruggles MB (2000). Basic Properties of Reference Crossply Carbon-Fibre Composites. Oak Ridge National Laboratory, U.S. Department of Energy.

[94] Belytschko T and Black T (1999). Elastic crack growth in finite elements with minimal remeshing. International Journal for Numerical Methods in Engineering; 45(5): pp. 60-620.

[95] Zhen-zhong Du (2009). eXtended Finite Element Method in Abaqus. (C) Dassault Systemes.

[96] Alnabelseya A (2012). Subchondral Bone Properties of Femoroacetabular Impingement Patients. M.A.Sc. Thesis, Carleton University.

[97] Abdul-Aziz A, Limbert G, Young PG and Beresford-West T (2007). Using ABAQUS with 3D imaging techniques for material characterization. ABAQUS Users' Conference.

[98] Nikishkov Y, Makeev A and Seon G (2013). Progressive fatigue damage simulation method for composites. International Journal of Fatigue; 48: pp. 266-279.

[99] Vivian L (2011). Damage Analysis on Composite Laminates under Fatigue Loading. M.Sc. Thesis, University of Padova.

[100] Gigliotti L (2012). Assessment of the Applicability of XFEM in Abaqus for Modeling Crack Growth in Rubber. M.Sc. Thesis, KTH School of Engineering Sciences.

[101]Levén M and Rickert D (2012). Stationary 3D Crack Analysis with Abaqus XFEM for Integrity Assessment of Subsea Equipment. M.Sc. Thesis, Chalmers University of Technology.

[102] Schneider CA, Rasb WS and Eliceiri KW (2012). NIH Image to ImageJ: 25 years of image analysis. Nature Methods; 9: pp. 671-675.

[103] Sezgin M and Sankur B (2004). Survey over image thresholding techniques and quantitative performance evaluation. Journal of Electronic Imaging; 13(1): pp. 146165. 
[104]Johnson E and Young P (2005). Simpleware: From 3D image to mesh in minutes. CSAR Focus, Edition 14 (Autumn - Winter); 13-15.

[105]Fang Q and Boas D (2009). Tetrahedral mesh generation from volumetric binary and gray-scale images. Proceedings of IEEE International Symposium on Biomedical Imaging, pp. 1142-1145.

[106] Yang Q and Cox B (2005). Cohesive models for damage evolution in laminated composites. International Journal of Fracture; 133: pp. 107-137.

[107]Drago A and Pindera M (2005). Micromechanical analysis of heterogeneous materials using FEMLAB. In: COMSOL Multiphysics User's Conference, Boston.

[108]Department of Defense Handbook (2002). Composite Materials Handbook; volume 3. Polymer Matrix Composites, materials Usage, Design and Analysis.

[109] Whitney J M, Browning C E, and Hoogsteden W (1982). A double cantilever beam test for characterizing mode I delamination of composite materials. Journal of Reinforced Plastics and Composites; 1(4): 297-313.

[110]De Moura MFSF, Campilho RDSG and Gonçalves JPM (2009). Pure mode II fracture characterization of composite bonded joints. International Journal of Solids Structures; 46: 1589-95.

[111]Benzeggagh ML and Kenane M (1996). Measurements of mixed-mode delamination fracture toughness of unidirectional glass/epoxy composites with mixed-mode bending apparatus. Composites Science and Technology; 56: 439-449.

[112]Blanco N, Gamstedt, EK, Asp LE and Costa J. (2004). Mixed-mode delamination growth in carbon-fibre composite laminates under cyclic loading. International Journal of Solids and Structures; 41(15): 4219-4235.

[113]Asp LE, Sjögren A and Greenhalgh E. (2001). Delamination growth and thresholds in a carbon/epoxy composite under fatigue loading. Journal of Composites Technology and Research; 23 (2): 55-68.

[114] Harper PW and Hallett SR (2010). A fatigue degradation law for cohesive interface elements- development and application to composite materials. International Journal of Fatigue; 32: 1774-1787.

[115]de Moura MFSF and Gonçalves JPM (2014). Development of a model for fatigue/fracture characterization of composite bonded joints under mode II loading. International Journal of Adhesion \& Adhesives; 54: 224-230.

[116]Paris P and Erdogan F (1963). A critical analysis of crack propagation laws. Journal of Basic Engineering, Transactions of the American Society of Mechanical Engineers; 85(4): 528-534.

[117] Schijve J (2008). Fatigue of structures and materials. Dordrecht: Springer. 
[118] Singh CV and Talreja R (2010). Evolution of Ply Cracks in Multidirectional Composite Laminates. International Journal of Solids and Structures; 47(10): 13381349.

[119]Prewitt JMS and Mendelsohn ML (1966). The analysis of cell images. Annals of the New York Academy of Sciences; 128(3): 1035-1053.

[120]Zack GW, Rogers WE and Latt SA (1977). Automatic measurement of sister chromatid exchange frequency. The journal of Histochemistry and Cytochemistry: official journal of the Histochemistry Society; 25: 741-753.

[121]Zhang J, Peng L, Zhao L and Fei B (2012). Fatigue delamination growth rates and thresholds of composite laminates under mixed mode loading. International Journal of Fatigue; 40: 7-15.

[122]Barenblatt GI (1959). Equilibrium cracks formed during brittle fracture rectilinear cracks in plane plates. Journal of Applied Mathematics and Mechanics; 23(4): pp. 1009-1029.

[123]Rose LRF (1987). Crack reinforcement by distributed springs. Journal of the Mechanics and Physics of Solids; 34: pp. 383-405.

[124]Roache PJ (2003), Verification and Validation in Computational Science and Engineering. Hermosa Publishers, Albuquerque. 


\section{Appendix A}

\section{Test Sample Specifications}

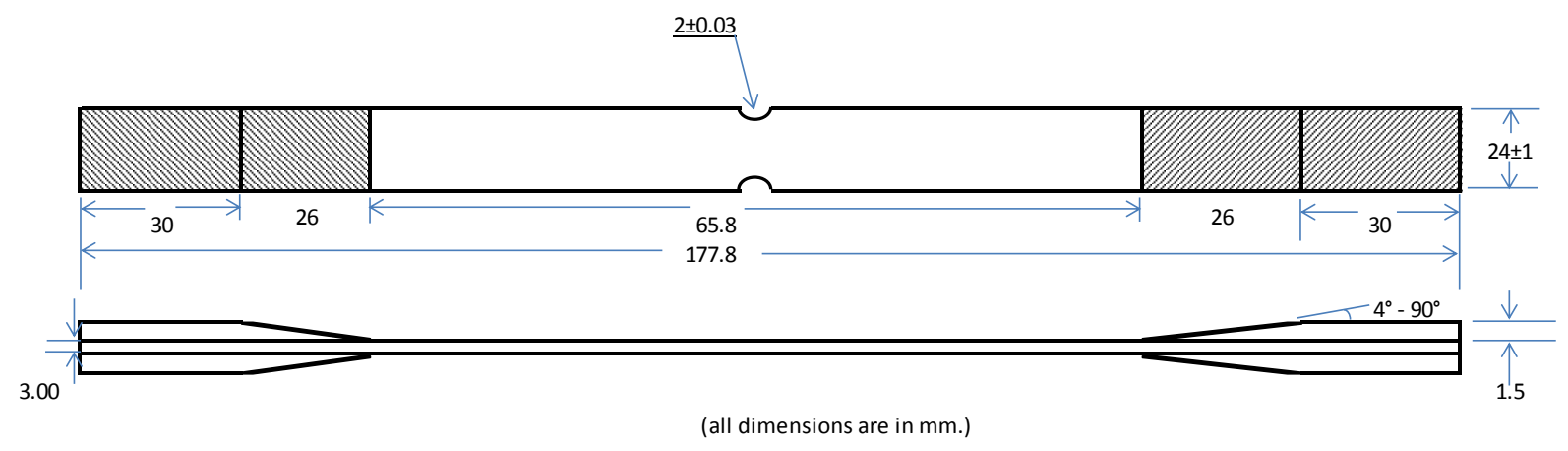

$\checkmark$ Ratio of width/diameter : 6

$\checkmark$ The ratio of diameter/height : 1.3

$\checkmark$ Configuration is cross-ply laminate stacking sequence $\left[0 / 90^{\circ} / 0^{\circ} / 90^{\circ}\right]$ s.

$\checkmark$ Number of plies: 8; featuring a height of $3.00 \mathrm{~mm}$.

$\checkmark$ Area of cross section is $72.00 \mathrm{~mm}^{2}$

Standards

$\checkmark$ ASTM Standard D5766/D5766M, "Standard Test Method for Open-Hole Tensile Strength of Polymer Matrix Composite Laminates". ASTM International, West Conshohocken, PA, 2011, DOI: 10.1520/D5766_D5766M-11.

$\checkmark$ ASTM Standard D7615, 2011, "Standard Practice for Open-Hole Fatigue Response of Polymer Matrix Composite Laminates". ASTM International, West Conshohocken, PA, 2011, DOI: 10.1520/D3039 -08. 


\section{Appendix B}

\section{Material: DA 409U/G35 150 Unidirectional Carbon/Epoxy Prepreg}

\section{Properties (supplier specifications sheet):}

$\begin{array}{ll}\text { Specific area: } & \left.150 \mathrm{~g} / \mathrm{m}^{2} \text { (may be supplied in } 300 \mathrm{~g} / \mathrm{m}^{2}\right) \\ \text { Thickness: } & \begin{array}{l}0.006 \text { inch/ply }(0.1524 \mathrm{~mm})-\text { corrected to } 0.375 \mathrm{~mm} \text { after } \\ \text { investigating the samples in lab. } \\ 48 \% \text { by weight }\end{array} \\ \begin{array}{l}\text { Resin Content: } \\ \text { Fibre volume fraction: }\end{array} & \begin{array}{l}0.57 \\ \text { Nominal laminate density: } \\ \text { Tow count: }\end{array} \\ \text { Fibre density: } & 1.96 \mathrm{~g} / \mathrm{cm}^{3} \\ & 1.77 \mathrm{~g} / \mathrm{cm}^{3} \\ \text { Tensile Modulus: } & 18.8 \mathrm{msi}(129.621 \mathrm{GPa}) \\ \text { Tensile Strength: } & 280 \mathrm{ksi}(1930.53 \mathrm{MPa}) \\ \text { Flexural Strength: } & 224 \mathrm{ksi}(1544.43 \mathrm{MPa}) \\ \text { Flexural Modulus: } & 17.9 \mathrm{msi}(123.416 \mathrm{GPa}) \\ \text { Beam Sheer: } & 13.8 \mathrm{msi}(95.147 \mathrm{GPa})\end{array}$

2. Properties (data from literature) [59]:

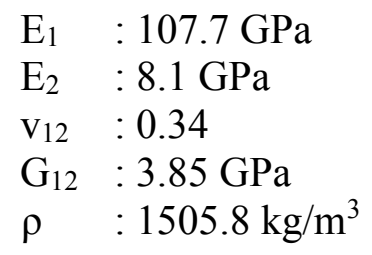

Engineering constants of each ply in a composite lay-up are listed in material \#1 table. If homogeneous isotropic material was assumed, material \#2 properties were used based on CLPT calculations of an 8-ply laminate; calculations are detailed in Appendix C.

Material \# 1

\begin{tabular}{|c|c|c|}
\hline $\mathrm{E} 1=107,700$ & $\mathrm{Nu} 12=0.34$ & $\mathrm{G} 12=3850$ \\
\hline $\mathrm{E} 2=8,100$ & $\mathrm{Nu} 13=0.34$ & $\mathrm{G} 13=3850$ \\
\hline $\mathrm{E} 3=8,100$ & $\mathrm{Nu} 23=0.5$ & $\mathrm{G} 23=1925$ \\
\hline
\end{tabular}

Material \# 2

\begin{tabular}{|c|}
\hline$E=175000$ \\
\hline$v=0.34$ \\
\hline
\end{tabular}




\section{Appendix C}

\section{CLT Calculations of Laminate Constants}

1. Unidirectional single lamina

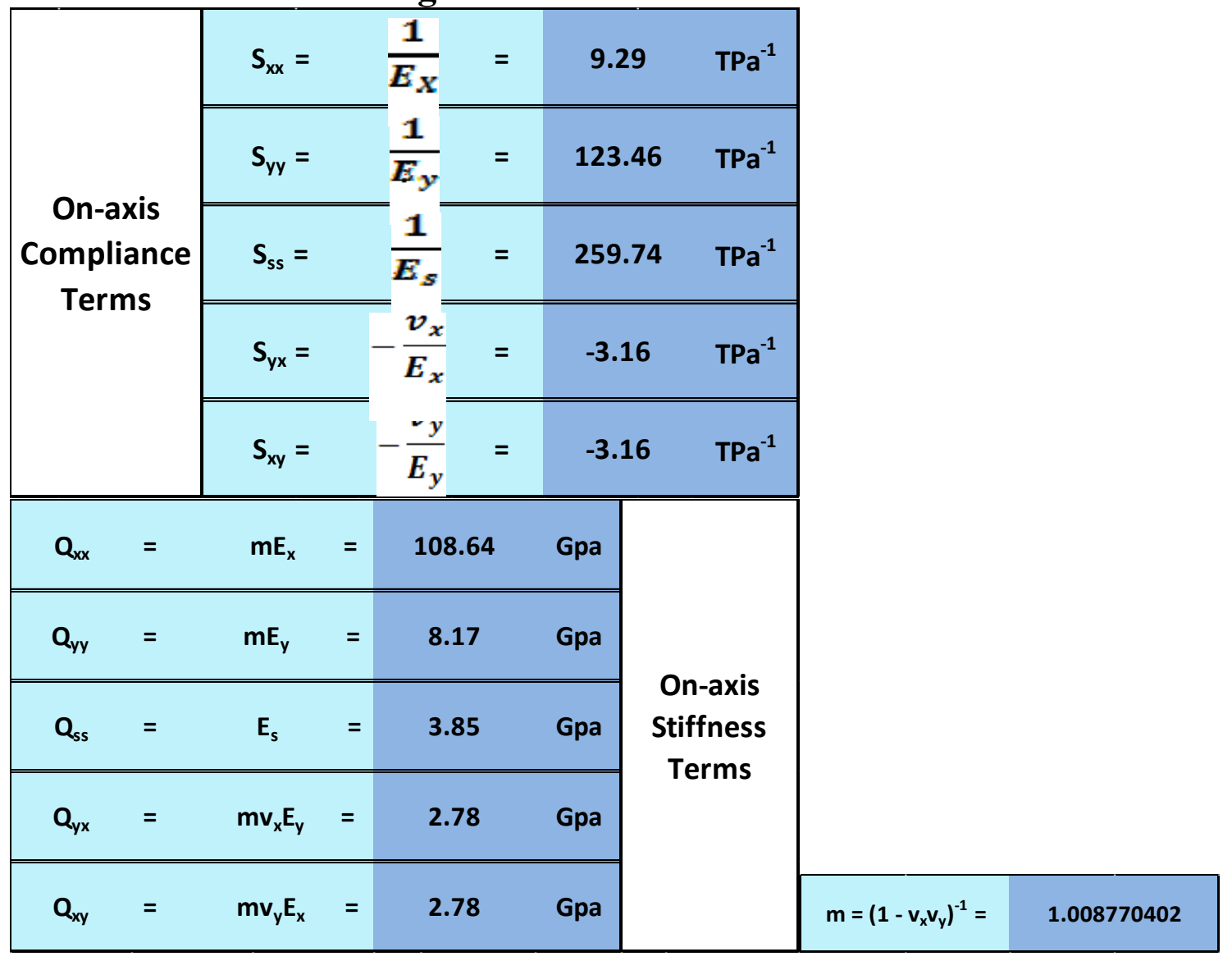

$\mathrm{U}, \mathrm{U}$ " terms

\begin{tabular}{|llll|}
\hline$U_{1}=1 / 8\left(3 Q_{x x}+3 Q_{y y}+2 Q_{x y}+4 Q_{s s}\right)$ & $=$ & 46.43 & $\mathrm{Gpa}$ \\
$U_{2}=1 / 2\left(Q_{x x}-Q_{y y}\right)$ & $=$ & 50.24 & $\mathrm{Gpa}$ \\
$U_{3}=1 / 8\left(Q_{x x}+Q_{y y}-2 Q_{x y}-4 Q_{s s}\right)$ & $=$ & 11.98 & $\mathrm{Gpa}$ \\
$U_{4}=1 / 8\left(Q_{x x}+Q_{y y}+6 Q_{x y}-4 Q_{s s}\right)$ & $=$ & 14.76 & $\mathrm{Gpa}$ \\
$U_{5}=1 / 2\left(U_{1}-U_{4}\right)$ & $=$ & 15.83 & $\mathrm{Gpa}$ \\
\hline$U^{\prime \prime}{ }_{1}=1 / 8\left(3 S_{x x}+3 S_{y y}+2 S_{x y}+S_{s s}\right)$ & $=$ & 81.46 & $\mathrm{TPa}^{-1}$ \\
$U^{\prime \prime}{ }_{2}=1 / 2\left(S_{x x}-S_{y y}\right)$ & $=$ & -57.09 & $\mathrm{TPa}^{-1}$ \\
$U^{\prime \prime}{ }_{3}=1 / 8\left(S_{x x}+S_{y y}-2 S_{x y}-S_{s s}\right)$ & $=$ & -112.49 & $\mathrm{TPa}^{-1}$ \\
$U^{\prime \prime}=1 / 8\left(S_{x x}+S_{y y}+6 S_{x y}-S_{s s}\right)$ & $=$ & -18.24 & $\mathrm{TPa}^{-1}$ \\
$U^{\prime \prime}=2\left(U^{\prime}{ }_{1}-U_{4}\right)$ & $=$ & 199.40 & $\mathrm{TPa}^{-1}$ \\
\hline
\end{tabular}




\subsection{Single ply on-axis stress-strain relationship}

$$
\begin{aligned}
& \sigma_{\mathrm{x}}=8 \mathrm{MPa} \quad \varepsilon_{\mathrm{x}}=2.117 \mathrm{E}-03 \\
& \sigma_{y}=5 \quad \mathrm{MPa} \quad \varepsilon_{\mathrm{y}}=5.206 \mathrm{E}-03 \\
& \sigma_{s}=3 \mathrm{MPa} \quad \varepsilon_{\mathrm{s}}=2.092 \mathrm{E}-03 \\
& \text { (i) } \quad\left[\begin{array}{c}
\varepsilon_{x} \\
\varepsilon_{y} \\
\varepsilon_{s}
\end{array}\right]=\left(\begin{array}{ccc}
S_{x x} & S_{x y} & 0 \\
S_{y x} & S_{y y} & 0 \\
0 & 0 & S_{s s}
\end{array}\right) \cdot\left(\begin{array}{c}
\sigma_{x} \\
\sigma_{y} \\
\sigma_{s}
\end{array}\right)= \\
& \left(\begin{array}{ccc}
9.29 \mathrm{E}-12 & -3.16 \mathrm{E}-12 & 0 \\
-3.16 \mathrm{E}-12 & 1.23 \mathrm{E}-10 & 0 \\
0 & 0 & 2.60 \mathrm{E}-10
\end{array}\right) \cdot\left(\begin{array}{l}
8 . \mathrm{E}+06 \\
5 . \mathrm{E}+06 \\
3 . \mathrm{E}+06
\end{array}\right)=\left(\begin{array}{l}
6.28 \mathrm{E}-05 \\
5.91 \mathrm{E}-04 \\
7.79 \mathrm{E}-04
\end{array}\right) \\
& \text { (ii) } \quad\left[\begin{array}{l}
\sigma_{x} \\
\sigma_{y} \\
\sigma_{s}
\end{array}\right)=\left(\begin{array}{ccc}
Q_{x x} & Q_{x y} & 0 \\
Q_{y x} & Q_{y y} & 0 \\
0 & 0 & Q_{s s}
\end{array}\right) \cdot\left(\begin{array}{c}
\varepsilon_{x} \\
\varepsilon_{y} \\
\varepsilon_{s}
\end{array}\right)= \\
& \left(\begin{array}{ccc}
1.09 \mathrm{E}+05 & 2.78 \mathrm{E}+03 & 0 \\
2.78 \mathrm{E}+03 & 8.17 \mathrm{E}+03 & 0 \\
0 & 0 & 3.85 \mathrm{E}+03
\end{array}\right) \cdot\left(\begin{array}{c}
2.117 \mathrm{E}-03 \\
5.206 \mathrm{E}-03 \\
2.092 \mathrm{E}-03
\end{array}\right)=\left(\begin{array}{c}
244.46 \\
48.42 \\
8.05
\end{array}\right) \begin{array}{l}
\mathrm{MPa} \\
\mathrm{MPa} \\
\mathrm{MPa}
\end{array} \\
& \begin{array}{r}
\varepsilon_{x}=\frac{1}{E_{x}} \sigma_{x}-\frac{v_{y}}{E_{y}} \sigma_{y}=6.2767 \mathrm{E}-05 \\
\varepsilon_{y}=-\frac{v_{x}}{E_{x}} \sigma_{x}+\frac{1}{E_{y}} \sigma_{y}=\quad 5.906 \mathrm{E}-04 \\
\varepsilon_{x}=\frac{1}{E_{s}} \sigma_{s}=7.792 \mathrm{E}-04
\end{array} \\
& \sigma_{x}=m E_{x}\left(\varepsilon_{x}+v_{y} \varepsilon_{y}\right)=244.46 \mathrm{MPa} \\
& \sigma_{y}=m E_{y}\left(v_{x} \varepsilon_{x}+\varepsilon_{y}\right)=\quad 48.42 \mathrm{MPa} \\
& \sigma_{s}=E_{x} \varepsilon_{s}=\quad 8.05 \mathrm{MPa}
\end{aligned}
$$

\subsection{Single ply off-axis stress-strain relationship}

\subsubsection{Off-axis rotation angle $=0$}

Off-axix orientation angle $\theta=0^{\circ}$

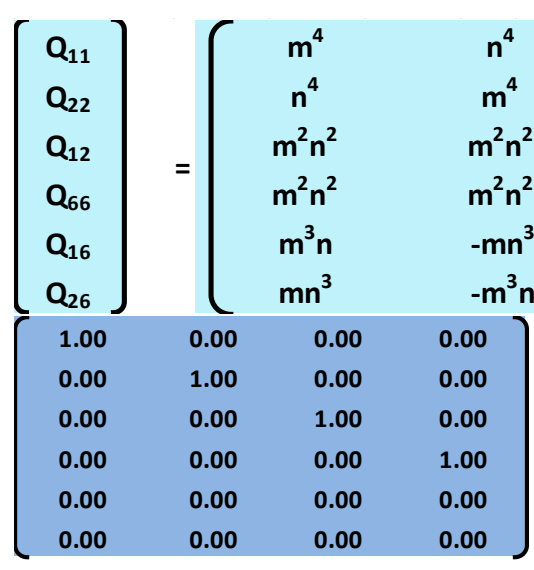

$m=\cos \theta=1.0000$

$\mathrm{n}=\sin \theta=\quad 0.0000$

$\left.\begin{array}{cc}\left.\begin{array}{cc}n=\sin \theta= \\ 2 m^{2} n^{2} \\ 2 m^{2} n^{2} & 4 m^{2} n^{2} \\ m^{4}+n^{4} & 4 m^{2} n^{2} \\ -2 m^{2} n^{2} & -4 m^{2} n^{2} \\ m n^{3}-m^{3} n & \left(m^{2}-n^{2}\right)^{2} \\ m^{3} n-m^{3} & 2\left(m n^{3}-m^{3} n\right) \\ 108.64 \\ 8.17 \\ 2.78 \\ 3.85\end{array}\right] \quad 2\left(m^{3} n-m n^{3}\right)\end{array}\right] \cdot\left[\begin{array}{l}Q_{x x} \\ Q_{y y} \\ Q_{x y} \\ Q_{s s}\end{array}\right]$




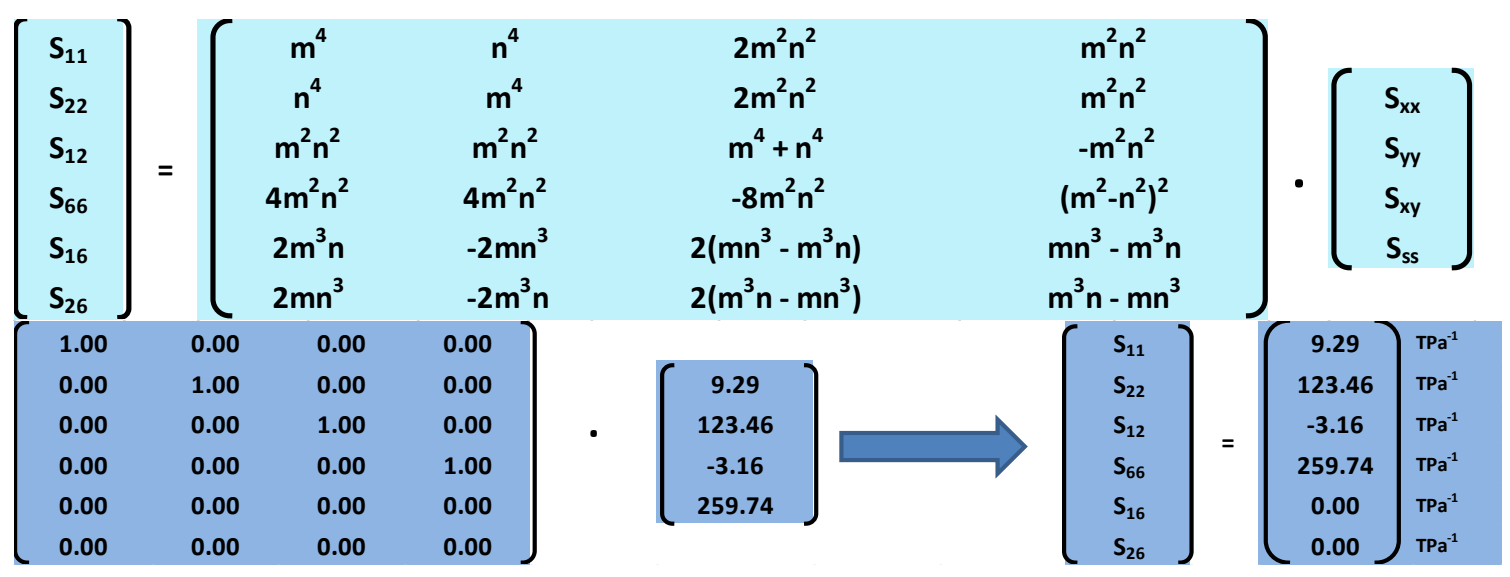

\subsubsection{Off-axis rotation angle $=90^{\circ}$}

Off-axix orientation angle $\theta=90^{\circ}$

$\left[\begin{array}{l}Q_{11} \\ Q_{22} \\ Q_{12} \\ Q_{66} \\ Q_{16} \\ Q_{26}\end{array}\right]$

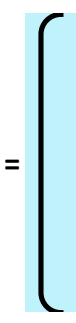

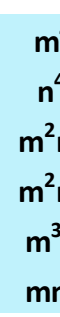

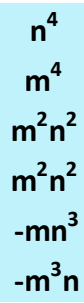

$m=\cos \theta=0.0000$ $n=\sin \theta=\quad 1.0000$

\begin{tabular}{|c|c|c|c|c|c|c|c|c|}
\hline 0.00 & 1.00 & 0.00 & 0.00 & & $\mathbf{Q}_{11}$ & & 8.17 & GPa \\
\hline 1.00 & 0.00 & 0.00 & 0.00 & 108.64 & $Q_{22}$ & & 108.64 & GPa \\
\hline 0.00 & 0.00 & 1.00 & 0.00 & 8.17 & $Q_{12}$ & - & 2.78 & GPa \\
\hline 0.00 & 0.00 & 0.00 & 1.00 & 2.78 & $Q_{66}$ & & 3.85 & GPa \\
\hline 0.00 & 0.00 & 0.00 & 0.00 & 3.85 & $\mathbf{Q}_{16}$ & & 0.00 & GPa \\
\hline 0.00 & 0.00 & 0.00 & 0.00 & & $Q_{26}$ & & 0.00 & GPa \\
\hline
\end{tabular}

\begin{tabular}{|c|c|c|c|c|c|c|c|}
\hline $\begin{array}{l}S_{11} \\
S_{22} \\
S_{12} \\
S_{66} \\
S_{16} \\
S_{26}\end{array}$ & $=$ & $\begin{array}{c}m^{4} \\
n^{4} \\
m^{2} n^{2} \\
4 m^{2} n^{2} \\
2 m^{3} n \\
2 m n^{3}\end{array}$ & $\begin{array}{c}n^{4} \\
m^{4} \\
m^{2} n^{2} \\
4 m^{2} n^{2} \\
-2 m n^{3} \\
-2 m^{3} n\end{array}$ & $\begin{array}{c}2 m^{2} n^{2} \\
2 m^{2} n^{2} \\
m^{4}+n^{4} \\
-8 m^{2} n^{2} \\
2\left(m n^{3}-m^{3} n\right) \\
2\left(m^{3} n-m n^{3}\right)\end{array}$ & $\begin{array}{c}m^{2} n^{2} \\
m^{2} n^{2} \\
-m^{2} n^{2} \\
\left(m^{2}-n^{2}\right)^{2} \\
m n^{3}-m^{3} n \\
m^{3} n-m n^{3}\end{array}$ & • & $\begin{array}{l}S_{x x} \\
S_{y y} \\
S_{x y} \\
S_{s s}\end{array}$ \\
\hline 0.00 & 1.00 & 0.00 & 0.00 & & $s_{11}$ & 123.46 & $\mathrm{TPa}^{-1}$ \\
\hline 1.00 & 0.00 & 0.00 & 0.00 & 9.29 & $S_{22}$ & 9.29 & $\mathrm{TPa}^{-1}$ \\
\hline 0.00 & 0.00 & 1.00 & 0.00 & 123.46 & $S_{12}$ & -3.16 & $\mathrm{TPa}^{-1}$ \\
\hline 0.00 & 0.00 & 0.00 & 1.00 & -3.16 & $S_{66}$ & 259.74 & $\mathrm{TPa}^{-1}$ \\
\hline 0.00 & 0.00 & 0.00 & 0.00 & 259.74 & $S_{16}$ & 0.00 & $\mathrm{TPa}^{-1}$ \\
\hline 0.00 & 0.00 & 0.00 & 0.00 & & $S_{26}$ & 0.00 & $\mathrm{TPa}^{-1}$ \\
\hline
\end{tabular}


1.2.3 Single ply off-axis engineering constants $\left(0^{\circ}\right)$

$$
\begin{aligned}
& \text { In-plane Poisson's ratio }=\quad v_{12}=-\frac{S_{12}}{S_{22}}= \\
& v_{21}=-\frac{S_{21}}{S_{11}}=\mathbf{0 . 3 4} \\
& \text { In-plane shear coupling coefficient }=\quad v_{61}=\frac{S_{61}}{S_{11}}= \\
& v_{62}=\frac{S_{62}}{S_{22}}= \\
& \text { In-plane normal coupling coefficient }=\quad v_{16}=-\frac{S_{16}}{S_{66}}= \\
& v_{26}=\frac{S_{26}}{S_{66}}=\mathbf{0 . 0 0} \\
& \text { In-plane longitudinal moduli }=\quad E_{1}=\frac{1}{S_{11}}= \\
& E_{2}=\frac{1}{S_{22}}=8.10 \quad \mathrm{Gpa} \\
& \text { In-plane shear modulus }=\quad E_{6}=\frac{1}{S_{66}}=
\end{aligned}
$$

1.2.4 Single ply off-axis engineering constants $\left(90^{\circ}\right)$

$$
\begin{aligned}
& \text { In-plane Poisson's ratio }=\quad v_{12}=-\frac{S_{12}}{S_{22}}= \\
& v_{21}=-\frac{S_{21}}{S_{11}} \quad=0.03 \\
& \text { In-plane shear coupling coefficient }=\quad v_{61}=\frac{S_{61}}{S_{11}}= \\
& v_{62}=\frac{S_{62}}{S_{22}}= \\
& \text { In-plane normal coupling coefficient }=\quad v_{16}=-\frac{S_{16}}{S_{66}}= \\
& v_{26}=\frac{S_{26}}{S_{66}}= \\
& \text { In-plane longitudinal moduli }=\quad E_{1}=\frac{1}{S_{11}}= \\
& E_{2}=\frac{1}{S_{22}}=107.70 \quad \text { Gpa } \\
& \text { In-plane shear modulus }=\quad E_{6}=\frac{1}{S_{66}}=
\end{aligned}
$$




\section{Symmetrical Laminated Plate}

\begin{tabular}{|cc|}
$m=$ total number of plies $=$ & 8 \\
\hline Stacking Sequence $:[0 / 90 / 0 / 90]_{s}$ & \\
\hline
\end{tabular}

\begin{tabular}{|c|c|c|c|}
$\mathbf{i}$ & \multicolumn{1}{c}{$\boldsymbol{\theta}_{\mathbf{i}}$} & \multicolumn{1}{c|}{$\mathbf{h}_{\mathbf{i}}(\mathrm{mm})$} & $\mathbf{V}_{\mathbf{f}, \mathbf{i}}$ \\
\hline 1 & 0 & 0.375 & 0.25 \\
\hline 2 & 90 & 0.375 & 0.25 \\
\hline 3 & 0 & 0.375 & 0.25 \\
\hline 4 & 90 & 0.375 & 0.25 \\
\hline
\end{tabular}

\begin{tabular}{|l|l|l|}
\hline$V_{1}^{*}=v_{f, 1} \cos \left(2 \theta_{1}\right)+v_{f, 2} \cos \left(2 \theta_{2}\right)+\ldots+v_{f, m / 2} \cos \left(2 \theta_{m / 2}\right)$ & $\mathbf{v}_{1}^{*}=0.0000$ \\
\cline { 2 - 3 }$V_{2}^{*}=v_{f, 1} \cos \left(4 \theta_{1}\right)+v_{f, 2} \cos \left(4 \theta_{2}\right)+\ldots+v_{f, m / 2} \cos \left(4 \theta_{m / 2}\right)$ & $\mathbf{v}_{2}^{*}=1.0000$ \\
\cline { 2 - 2 }$V_{3}^{*}=v_{f, 1} \sin \left(2 \theta_{1}\right)+v_{f, 2} \sin \left(2 \theta_{2}\right)+\ldots+v_{f, m / 2} \sin \left(2 \theta_{m / 2}\right)$ & $\mathbf{v}_{3}^{*}=0.0000$ \\
\cline { 2 - 2 }$V_{4}^{*}=v_{f, 1} \sin \left(4 \theta_{1}\right)+v_{f, 2} \sin \left(4 \theta_{2}\right)+\ldots+v_{f, m / 2} \sin \left(4 \theta_{m / 2}\right)$ & $\mathbf{v}_{4}^{*}=0.0000$ \\
\hline
\end{tabular}

\subsection{Normalized stiffness terms $\left(\mathrm{A}_{\mathrm{ij}} / \mathrm{h}\right)$}

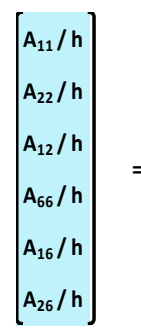

\{
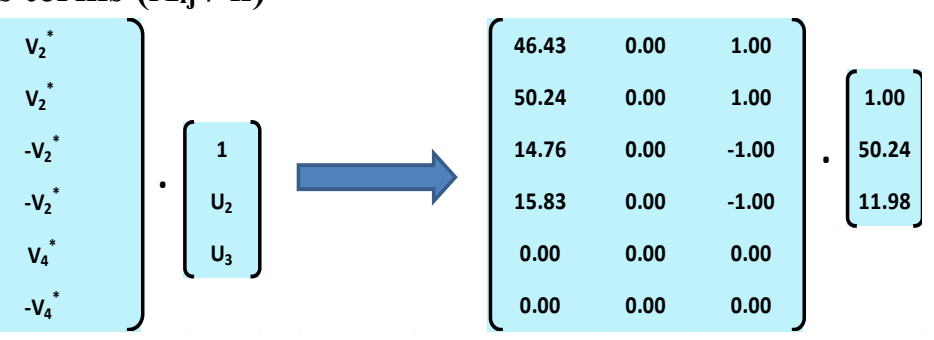

$$
\left[\begin{array}{l}
A_{11} / h \\
A_{22} / h \\
A_{12} / h \\
A_{66} / h \\
A_{16} / h \\
A_{26} / h
\end{array}\right]
$$

$=\left[\begin{array}{c|c}\mathbf{5 8 . 4 1} & \mathrm{GPa} \\ \mathbf{5 8 . 4 1} & \mathrm{GPa} \\ \mathbf{2 . 7 8} & \mathrm{GPa} \\ \mathbf{3 . 8 5} & \mathrm{GPa} \\ \mathbf{0 . 0 0} & \mathrm{GPa} \\ \mathbf{0 . 0 0} & \mathrm{GPa}\end{array}\right.$

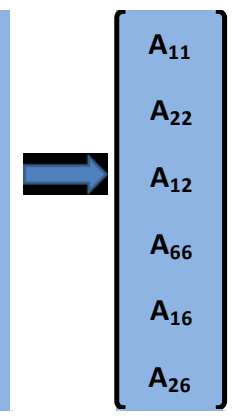

$=\left[\begin{array}{c|l}\mathbf{1 7 5 . 2 2} \\ 175.22 & \mathrm{MPa} \\ 8.33 & \mathrm{MPa} \\ 11.55 & \mathrm{MPa} \\ 0.00 & \mathrm{MPa} \\ 0.00 & \mathrm{MPa}\end{array}\right.$

2.2 Normalized compliance terms $\left(a_{i j} * h\right)$

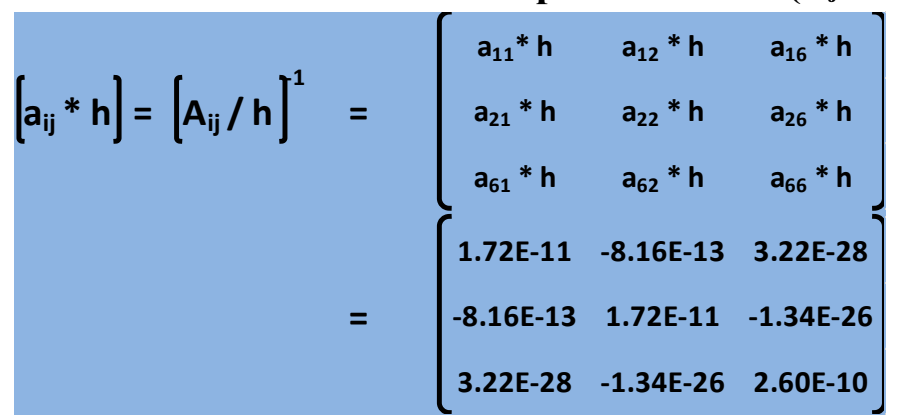

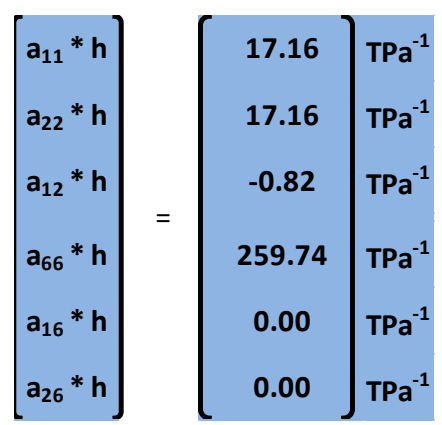




\subsection{Moment-curvature stiffness matrix $\left(\mathrm{D}_{\mathrm{ij}}\right)$}

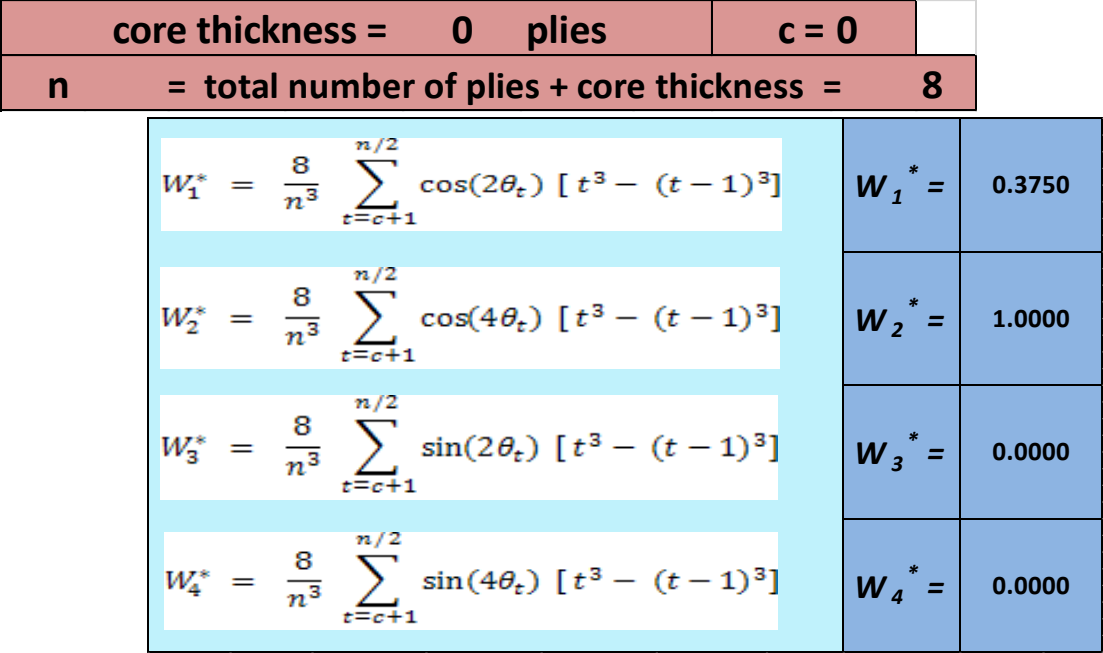

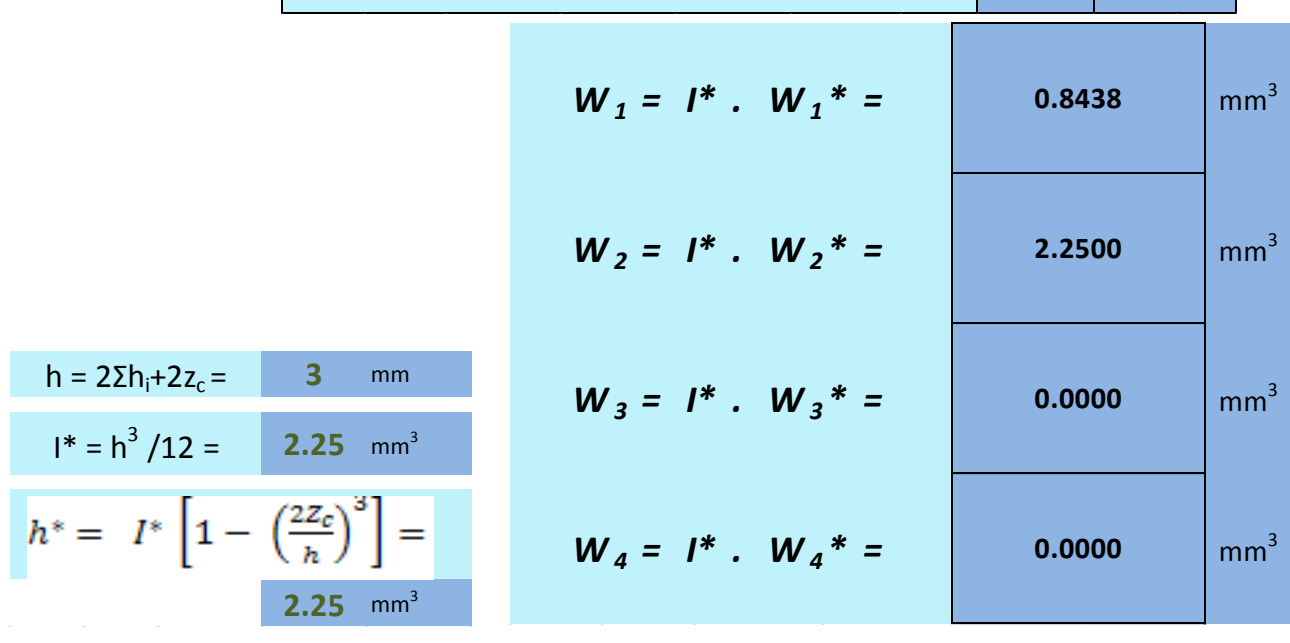

$$
\left[\begin{array}{l}
D_{11} \\
D_{22} \\
D_{12} \\
D_{66} \\
D_{16} \\
D_{26}
\end{array}\right]=\left[\begin{array}{ccc}
U_{1} & W_{1} & W_{2} \\
U_{2} & -W_{1} & W_{2} \\
U_{4} & 0 & -W_{2} \\
U_{5} & 0 & -W_{2} \\
0 & 1 / 2 W_{3} & W_{4} \\
0 & 1 / 2 W_{3} & -W_{4}
\end{array}\right] \cdot \cdot\left[\begin{array}{l}
h^{*} \\
U_{2} \\
U_{3}
\end{array}\right]
$$

$\left.\begin{array}{rrr}4.64 \mathrm{E}+10 & 8.44 \mathrm{E}-10 & 2.25 \mathrm{E}-09 \\ 4.64 \mathrm{E}+10 & -8.44 \mathrm{E}-10 & 2.25 \mathrm{E}-09 \\ 1.48 \mathrm{E}+10 & 0 & -2.25 \mathrm{E}-09 \\ 1.58 \mathrm{E}+10 & 0 & -2.25 \mathrm{E}-09 \\ 0 & 4.31 \mathrm{E}-26 & -1.72 \mathrm{E}-25 \\ 0 & 4.31 \mathrm{E}-26 & 1.72 \mathrm{E}-25\end{array}\right] \quad \bullet \quad\left[\begin{array}{l}2.25 \mathrm{E}-09 \\ 5.02 \mathrm{E}+10 \\ 1.20 \mathrm{E}+10\end{array}\right]\left[\begin{array}{l}\mathrm{D}_{11} \\ \mathrm{D}_{22} \\ \mathrm{D}_{12} \\ \mathrm{D}_{66} \\ \mathrm{D}_{16} \\ \mathrm{D}_{26}\end{array}\right]=\left[\begin{array}{r}173.80 \\ 89.03 \\ 6.25 \\ 8.66 \\ 0.00 \\ \mathrm{~N} \cdot \mathrm{m} \cdot \mathrm{N} \\ 0.00 \\ \mathrm{~N} \cdot \mathrm{m} \\ \mathrm{N} \cdot \mathrm{m}\end{array}\right.$




$\left[D_{i j}\right]=\left[\begin{array}{lll}D_{11} & D_{12} & D_{16} \\ D_{21} & D_{22} & D_{26} \\ D_{61} & D_{62} & D_{66}\end{array}\right]=\left[\begin{array}{lllll}173.80 & 6.25 & 0.00 & \text { N.m } \\ 6.25 & 89.03 & 0.00 & \text { N.m } \\ 0.00 & 0.00 & 8.66 & \text { N.m }\end{array}\right]$

\subsection{Moment-curvature compliance matrix $\left(\mathrm{d}_{\mathrm{ij}}\right)$}

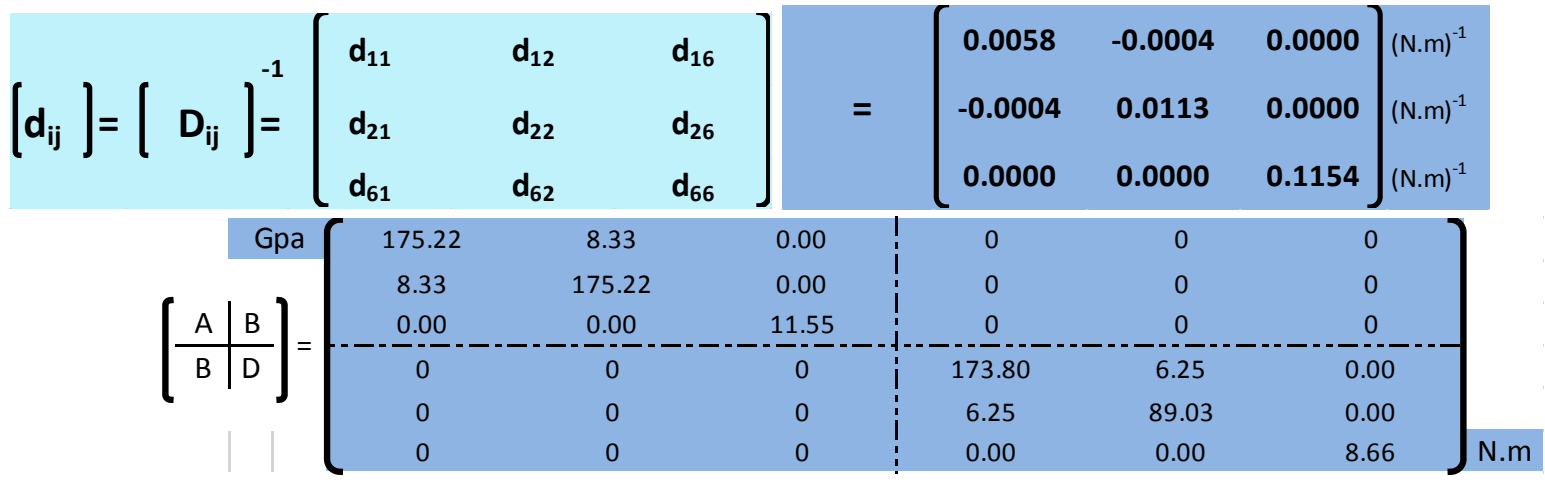

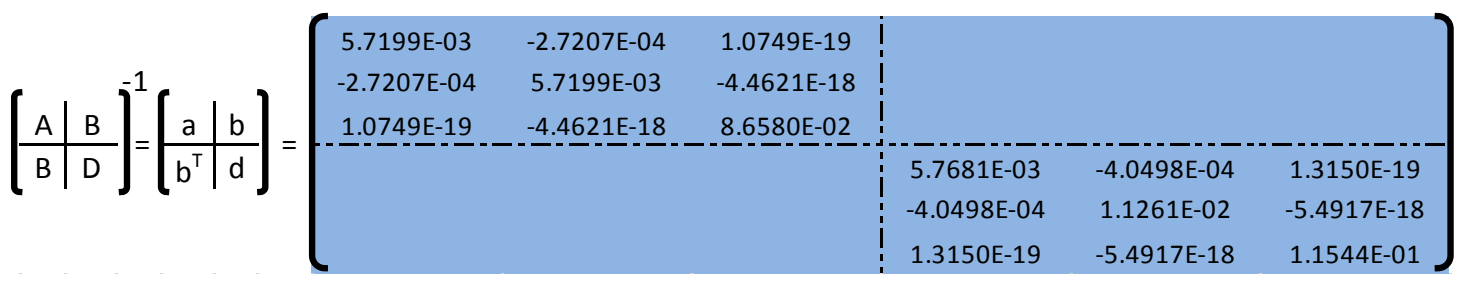

\begin{tabular}{|c|c|c|}
\hline \multicolumn{3}{|c|}{ Normalized Engineering constants } \\
\hline In-plane Poisson's ratio $=$ & $=\mathrm{v} 12=$ & 0.05 \\
\hline & $\mathrm{v} 21=$ & 0.05 \\
\hline \multirow[t]{2}{*}{ In-plane shear coupling coefficient } & $=v 61=$ & 0.00 \\
\hline & $v 62=$ & 0.00 \\
\hline \multirow{2}{*}{ In-plane normal coupling coefficient } & $=v 16=$ & 0.00 \\
\hline & $\mathrm{v} 26=$ & 0.00 \\
\hline \multirow[t]{2}{*}{ In-plane longitudinal moduli $=$} & $\mathrm{E} 1=$ & 58.28 \\
\hline & $\mathrm{E} 2=$ & 58.28 \\
\hline In-plane shear modulus $=$ & $\mathrm{E} 6=$ & 3.85 \\
\hline
\end{tabular}




\section{Appendix D}

\section{MATLAB Code for 3D Plotting of MicroCT scans}

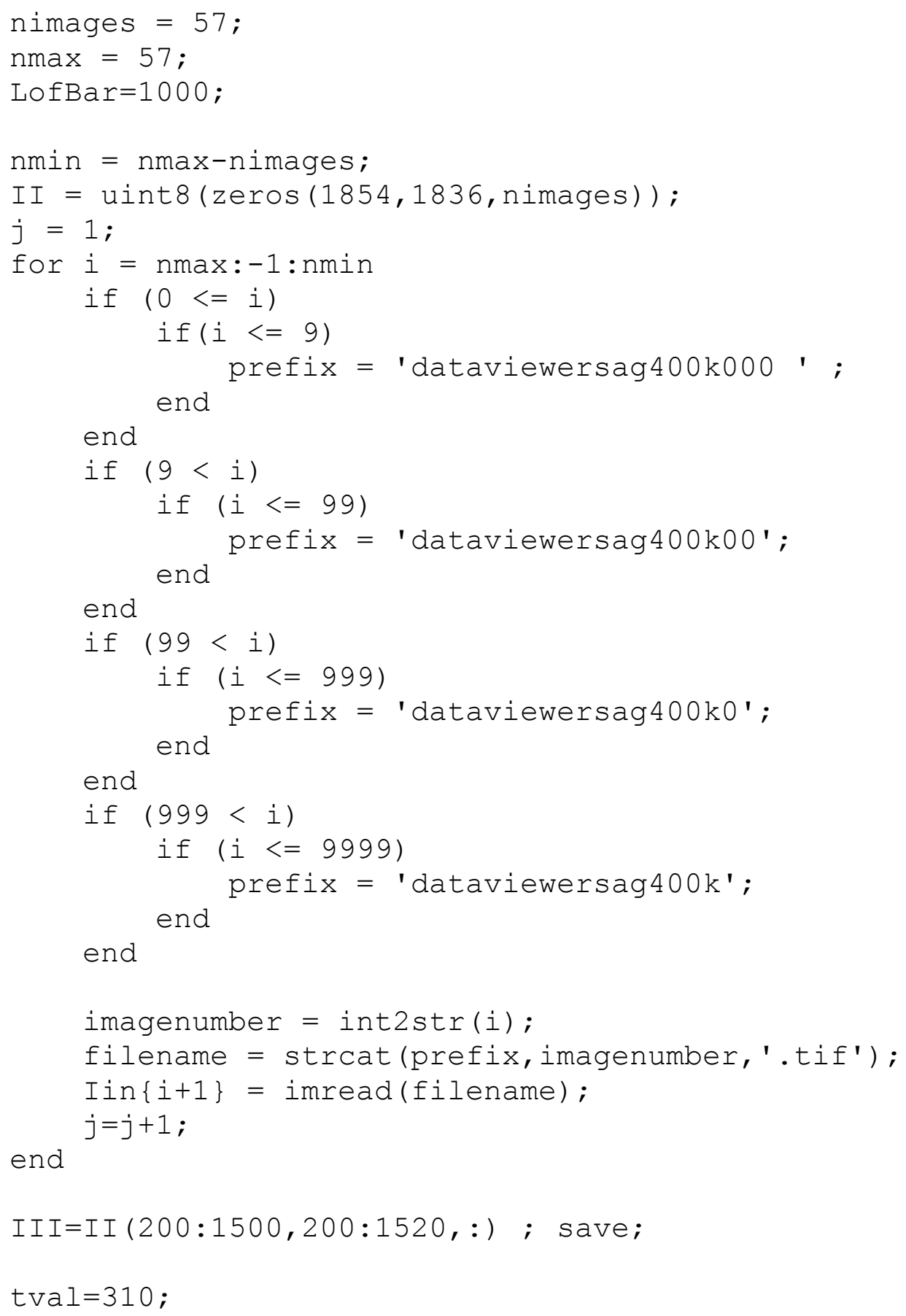


othresholding value; higher makes image brighter lower makes image darker

onofI=57; \%number of images

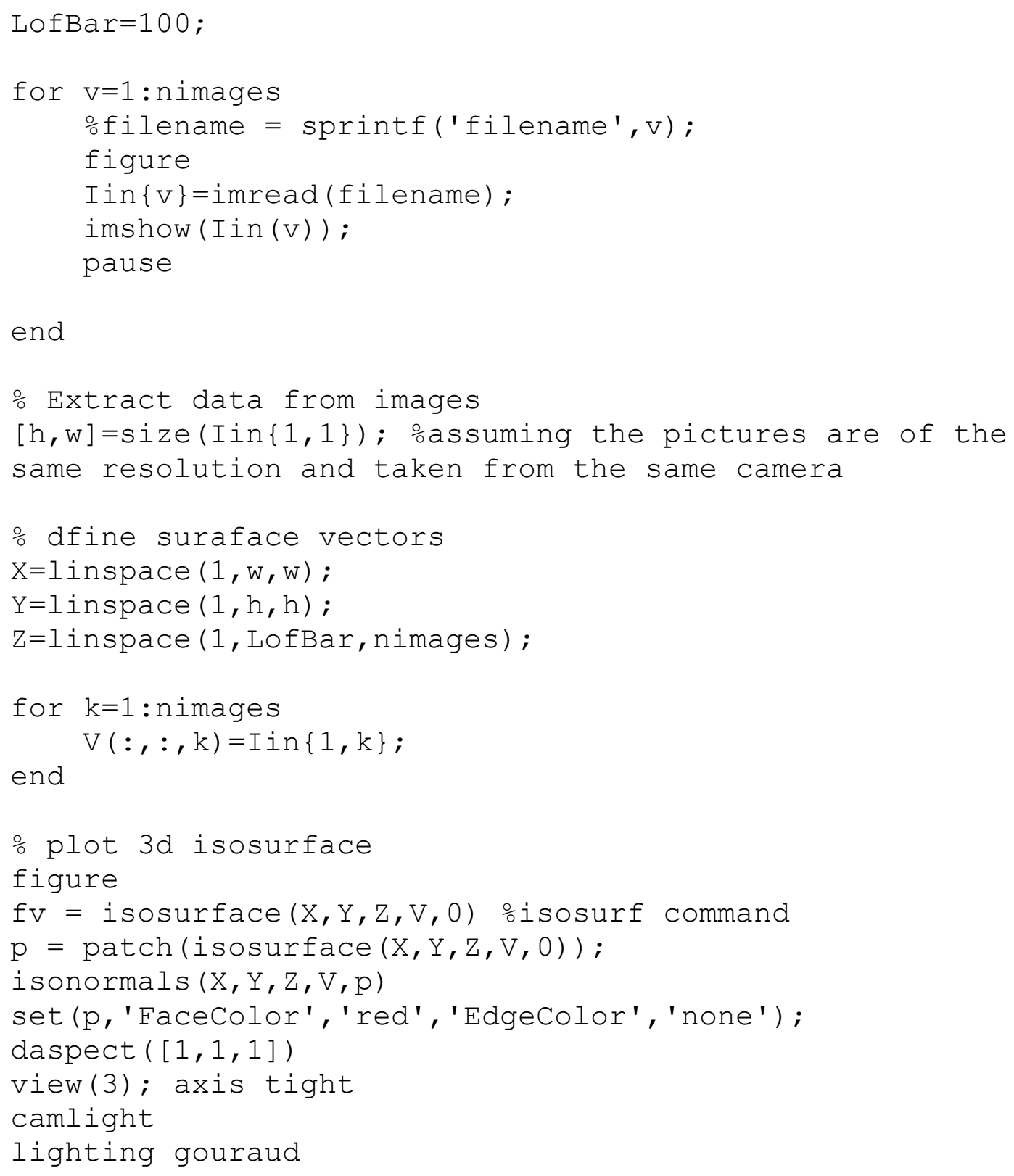

